\title{
Palladium-Catalyzed Ylidyl-Carbonylation of Aryl Halides to Produce $\alpha$-Acylphosphoranes
}

\author{
Xiaojun $\mathrm{Guo}^{\dagger}$, Wei Ma ${ }^{\dagger}$, Dong Xue ${ }^{* \dagger}$, Chao Wang ${ }^{\dagger}$ and Jianliang Xiao ${ }^{* \dagger+}$ \\ ${ }^{\dagger}$ Key Laboratory of Applied Surface and Colloid Chemistry (Shaanxi Normal University), \\ Ministry of Education and School of Chemistry and Chemical Engineering, Shaanxi Normal \\ University, Xi'an, 710062, China \\ $\ddagger$ Department of Chemistry, Liverpool Centre for Materials and Catalysis, University of \\ Liverpool, Liverpool, L69 7ZD, UK
}

xuedong_welcome@snnu.edu.cn; j.xiao@liverpool.ac.uk

\section{Contents}

1. General information

2. Optimation of reaction conditions 


\section{General information.}

All solvents were used in these experiments were reagent grade or better. 1,4-dioxane was refluxed over $\mathrm{CaH}_{2}$ and distilled under $\mathrm{N}_{2}$ atmosphere. (9,9-dimethyl-9H-xanthene-4,5-diyl)bis(diphenylphosphine) and $\mathrm{PdCl}_{2}$ were purchased from commercial suppliers and used without further purification. Some stabilized phosphorus ylides were synthesised by the methods reported in the literatures. ${ }^{1} \mathrm{H}$ NMR spectra were recorded on a Bruker Advance 400 (400 MHz) or $600(600 \mathrm{MHz}) \mathrm{NMR}$ spectrometer and reported in units of parts per million (ppm) relative to tetramethyl silane $(\delta 0 \mathrm{ppm})$ or $\mathrm{CDCl}_{3}(\delta 7.26 \mathrm{ppm})$. Multiplicities are given as: $\mathrm{s}$ (singlet), $\mathrm{d}$ (doublet), $\mathrm{t}$ (triplet), q (quartet), m (multiplet), dd (doublets of doublet), dt (doublets of triplet) or td (triplets of doublet). Coupling constants were reported as a $J$ value in Hz. ${ }^{13} \mathrm{C}$ NMR spectra were recorded on a Bruker Advance 400 $(100 \mathrm{MHz})$ or $600(150 \mathrm{MHz}) \mathrm{NMR}$ spectrometer and reported in ppm relative to $\mathrm{CDCl}_{3}(\delta 77.0 \mathrm{ppm}) .{ }^{31} \mathrm{P}$ NMR spectra were recorded on a Bruker Advance 400 (162 $\mathrm{MHz}$ ) NMR spectrometer and reported in ppm. Analytical thin-layer chromatography (TLC) was conducted with TLC Silica gel 60 F254 (Merck) and plates were revealed under UV irradiation. Column chromatography was performed on silica gel 200-300 mesh. Flash chromatography was performed with freshly distilled solvents. HRMS (ESI) were performed on Fourier Transform Ion Cyclotron Resonance Mass Spectrometer. Melting points were determined by using "X-T5B" melting point apparatus and were uncorrected. Microwave reactions were performed on Biotage $\left(\right.$ Initiator $\left.^{+}\right)$microwave reactor.

\section{Optimation of reaction conditions.}

\subsection{The optimization of reaction conditions for substituted aryl iodide.}

\subsubsection{Typical reaction procedure for the optimization of reaction conditions.}

The reaction was carried out in an autoclave containing a $10 \mathrm{~mL}$ Teflon reaction tube. Pd source $(0.01 \mathrm{mmol}, 2 \mathrm{~mol} \%)$, ligand $(0.02 \mathrm{mmol}, 4 \mathrm{~mol} \%)$ and a magnetic stir bar were placed in the tube, which was then capped with a stopper. Then, methyl 4-iodobenzoate 1 ( $0.5 \mathrm{mmol}, 1$ equiv), methyl 2-(triphenylphosphoranylidene)acetate 
2 (0.5 mmol, 1 equiv), base ( $0.5 \mathrm{mmol}, 1$ equiv), and solvent ( $3 \mathrm{~mL})$ were added to the tube. The tube was placed in the autoclave. Once sealed, the autoclave was purged three times with $\mathrm{CO}$ at room temperature and then pressurized to 6 atm with $\mathrm{CO}$ at room temperature and heated in an oil bath at $100{ }^{\circ} \mathrm{C}$ for 12 hours. The autoclave was then cooled to room temperature and vented to discharge the excess $\mathrm{CO}$. The mixture was filtered by celite and concentrated in vасио. The crude product was purified by column chromatography on silica gel using a mixture of ethyl acetate and petroleum ether as eluent to give the $\alpha$-acylphosphoranes. Structures of ligand screened in this paper are shown in Scheme S1.

Table S1: Background reactions, screening ligands, bases and Pd sources. ${ }^{\text {a }}$

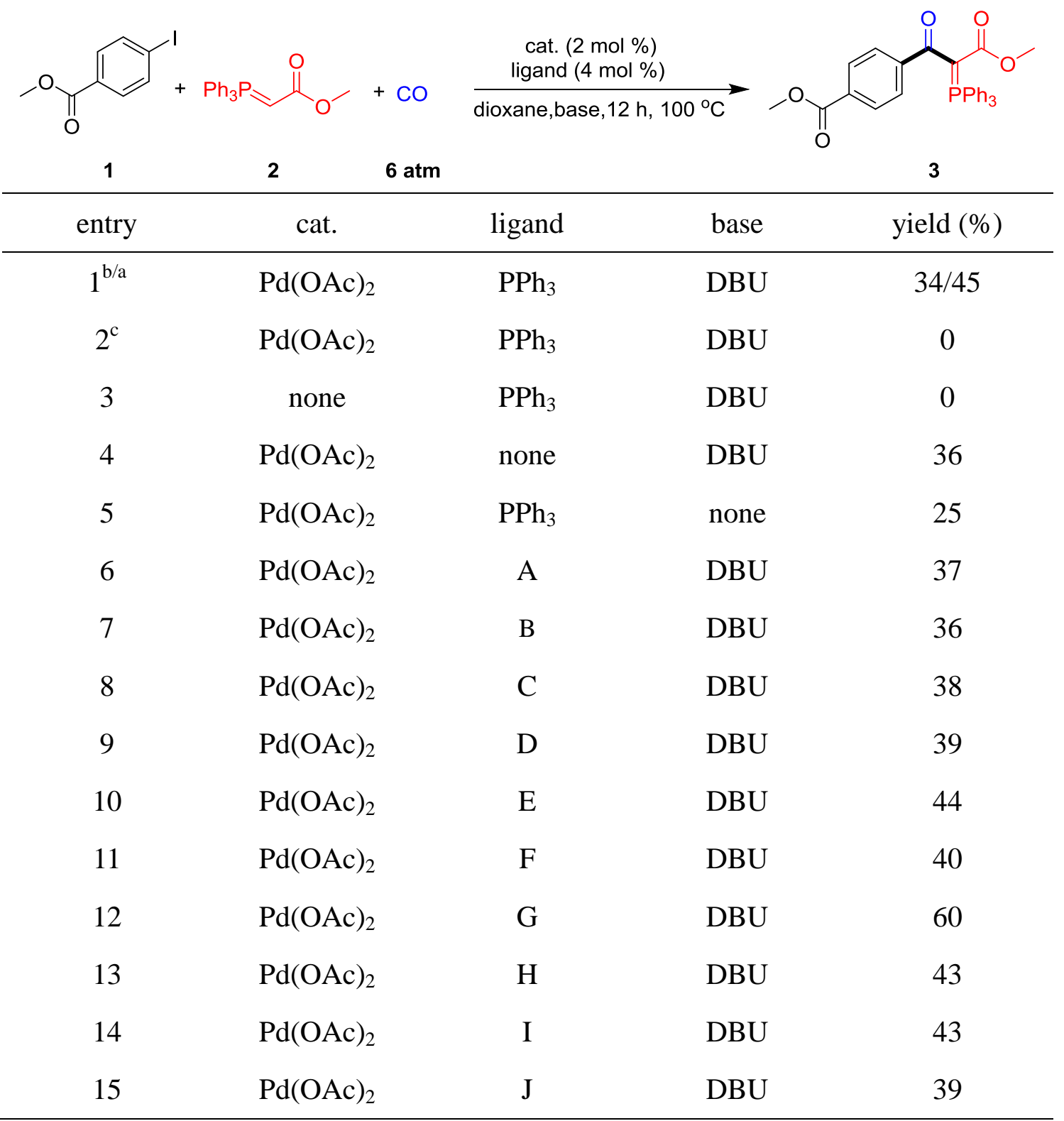




\begin{tabular}{|c|c|c|c|c|}
\hline 16 & $\mathrm{Pd}(\mathrm{OAc})_{2}$ & $\mathrm{~K}$ & DBU & 46 \\
\hline 17 & $\mathrm{Pd}(\mathrm{OAc})_{2}$ & $\mathrm{~L}$ & DBU & 27 \\
\hline 18 & $\mathrm{Pd}(\mathrm{OAc})_{2}$ & M & DBU & 30 \\
\hline 19 & $\mathrm{Pd}(\mathrm{OAc})_{2}$ & $\mathrm{~N}$ & DBU & 30 \\
\hline 20 & $\mathrm{Pd}(\mathrm{OAc})_{2}$ & $\mathrm{O}$ & DBU & 9 \\
\hline 21 & $\mathrm{Pd}(\mathrm{OAc})_{2}$ & $\mathrm{P}$ & $\mathrm{DBU}$ & 39 \\
\hline 22 & $\mathrm{Pd}(\mathrm{OAc})_{2}$ & Q & DBU & 4 \\
\hline 23 & $\mathrm{Pd}(\mathrm{OAc})_{2}$ & $\mathrm{G}$ & $\mathrm{KOH}$ & 14 \\
\hline 24 & $\mathrm{Pd}(\mathrm{OAc})_{2}$ & $\mathrm{G}$ & $\mathrm{NaOH}$ & 33 \\
\hline 25 & $\mathrm{Pd}(\mathrm{OAc})_{2}$ & $\mathrm{G}$ & $\mathrm{NEt}_{3}$ & 55 \\
\hline 26 & $\mathrm{Pd}(\mathrm{OAc})_{2}$ & $\mathrm{G}$ & $\mathrm{CH}_{3} \mathrm{CH}_{2} \mathrm{ONa}$ & 20 \\
\hline 27 & $\mathrm{Pd}(\mathrm{OAc})_{2}$ & $\mathrm{G}$ & $\mathrm{KF}$ & 23 \\
\hline 28 & $\mathrm{Pd}(\mathrm{OAc})_{2}$ & G & $\mathrm{Na}_{2} \mathrm{CO}_{3}$ & 37 \\
\hline 29 & $\mathrm{Pd}(\mathrm{OAc})_{2}$ & $\mathrm{G}$ & $\mathrm{K}_{3} \mathrm{PO}_{4}$ & 59 \\
\hline 30 & $\mathrm{Pd}(\mathrm{OAc})_{2}$ & $\mathrm{G}$ & $\mathrm{NaHCO}_{3}$ & 40 \\
\hline 31 & $\mathrm{Pd}(\mathrm{OAc})_{2}$ & $\mathrm{G}$ & $\mathrm{KHCO}_{3}$ & 66 \\
\hline 32 & $\mathrm{Pd}(\mathrm{OAc})_{2}$ & $\mathrm{G}$ & $\mathrm{CH}_{3} \mathrm{COONa}$ & 17 \\
\hline 33 & $\mathrm{Pd}(\mathrm{OAc})_{2}$ & G & $\mathrm{CH}_{3} \mathrm{COOK}$ & 3 \\
\hline 34 & $\mathrm{Pd}(\mathrm{OAc})_{2}$ & $\mathrm{G}$ & Pyridine & 31 \\
\hline 35 & $\mathrm{Pd}(\mathrm{OAc})_{2}$ & $\mathrm{G}$ & $\mathrm{K}_{2} \mathrm{CO}_{3}$ & 76 \\
\hline 36 & $\mathrm{PdCl}_{2}$ & $\mathrm{G}$ & $\mathrm{K}_{2} \mathrm{CO}_{3}$ & 81 \\
\hline 37 & $\mathrm{Pd}(\mathrm{PhCN})_{2} \mathrm{Cl}_{2}$ & $\mathrm{G}$ & $\mathrm{K}_{2} \mathrm{CO}_{3}$ & 73 \\
\hline 38 & $\mathrm{Pd}(\mathrm{MeCN})_{2} \mathrm{Cl}_{2}$ & $\mathrm{G}$ & $\mathrm{K}_{2} \mathrm{CO}_{3}$ & 76 \\
\hline
\end{tabular}


Scheme S1: Structures of ligands.

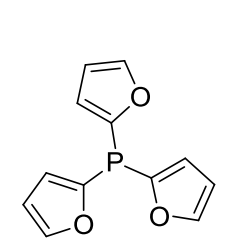

A<smiles>COc1ccc(P(c2ccc(OC)cc2)c2ccc(OC)cc2)cc1</smiles>

F<smiles>C1CCC(P(CCCP(C2CCCCC2)C2CCCCC2)C2CCCCC2)CC1</smiles>

$\mathbf{J}$<smiles>C1CCC(P(C2CCCCC2)C2CCCCC2)CC1</smiles>

$\mathbf{N}$<smiles>CC(C)c1cc(C(C)C)c(-c2ccccc2P(C(C)(C)C)C(C)(C)C)c(C(C)C)c1</smiles>

B<smiles>CC(C)(C)P(C(C)(C)C)C(C)(C)C</smiles>

C<smiles>c1ccc(-c2ccccc2P(C2CCCCC2)C2CCCCC2)cc1</smiles>

D

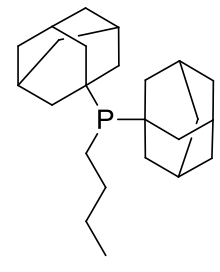

$\mathbf{E}$<smiles>CC1(C)c2cccc(P)c2Oc2c(-c3ccccc3)cccc21</smiles>

G<smiles>c1ccc(P(CCP(c2ccccc2)c2ccccc2)c2ccccc2)cc1</smiles>

$\mathbf{K}$<smiles>Pc1cccc(P)c1P</smiles>

$\mathbf{O}$<smiles>CC(C)(C)P(c1cccc(P(C(C)(C)C)C(C)(C)C)c1)P(C(C)(C)C)C(C)(C)C</smiles>

H<smiles>Pc1ccccc1-c1ccccc1-c1ccccc1</smiles>

$\mathbf{L}$<smiles>c1ccc(P(c2ccccc2)c2ccccc2P(c2ccccc2)c2ccccc2)cc1</smiles>

P<smiles>CC(C)(C)P(Cc1ccccc1CP(C(C)(C)C)C(C)(C)C)C(C)(C)C</smiles>

I<smiles>c1ccc(P(CCCP(c2ccccc2)c2ccccc2)c2ccccc2)cc1</smiles>

M

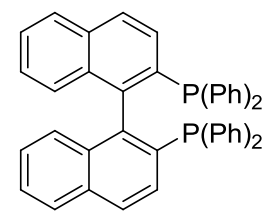

Q

Table S2: Screening of solvents. ${ }^{\text {a }}$

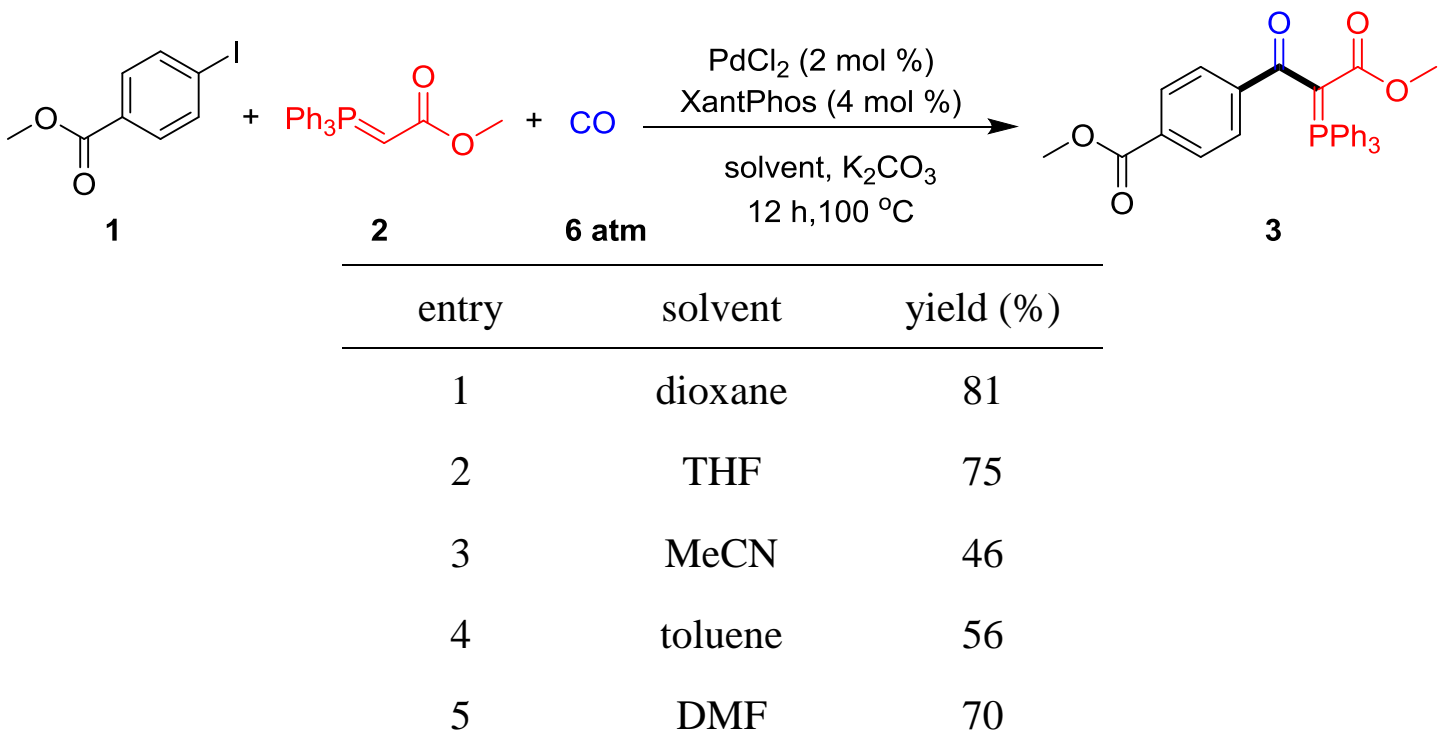

${ }^{\text {a }}$ Yield determined by ${ }^{1} \mathrm{H}$ NMR with 1, 3, 5-trimethoxybenzene as internal standard. 
Table S3: Screening the pressure of $\mathrm{CO} .{ }^{\mathrm{a}}$

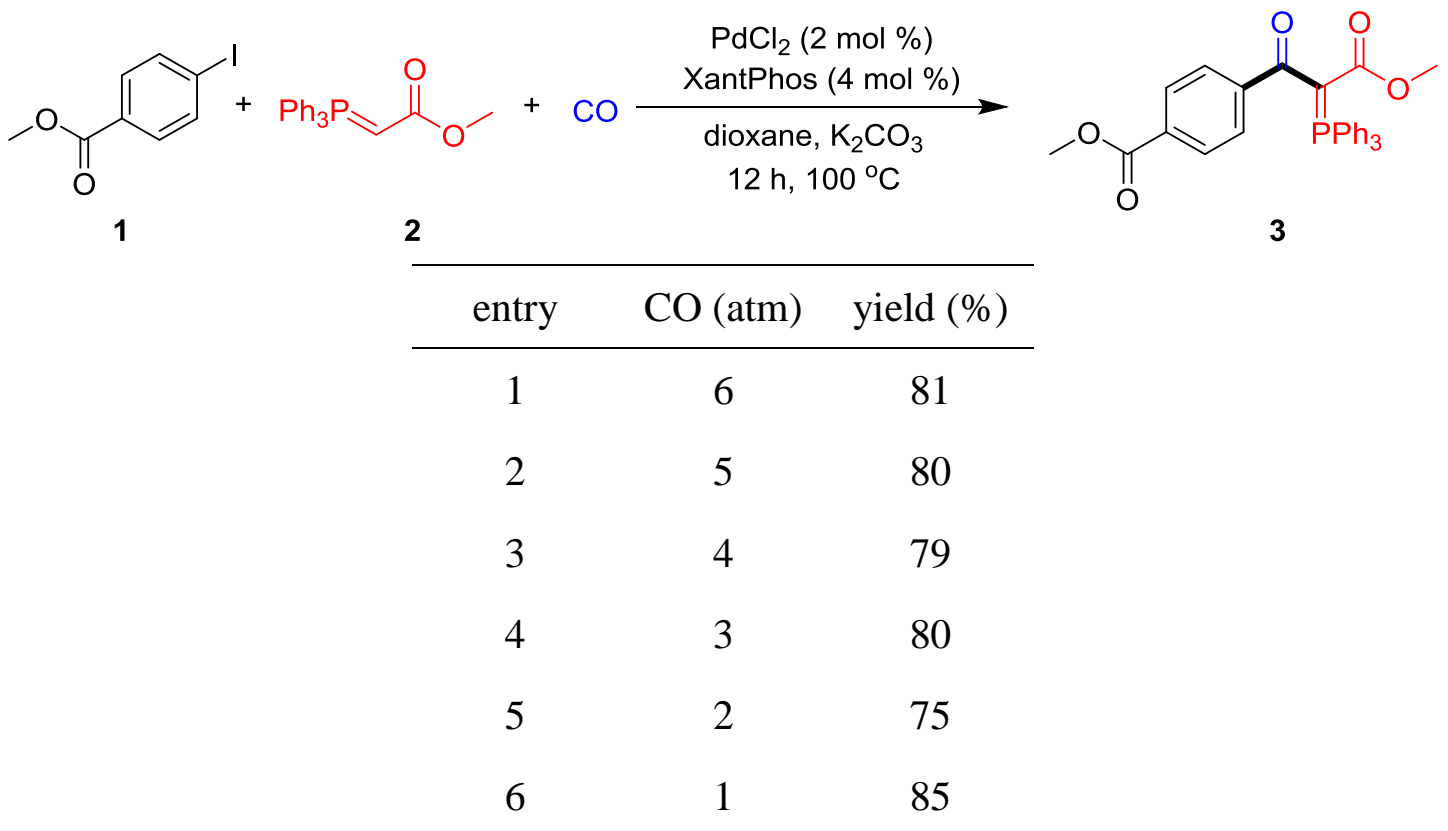

${ }^{\text {a }}$ Yield determined by ${ }^{1} \overline{\mathrm{H} \mathrm{NMR} \text { with 1, 3, 5-trimethoxybenzene }}$ as internal standard.

Table S4: Screening the reaction temperatures. ${ }^{\text {a }}$

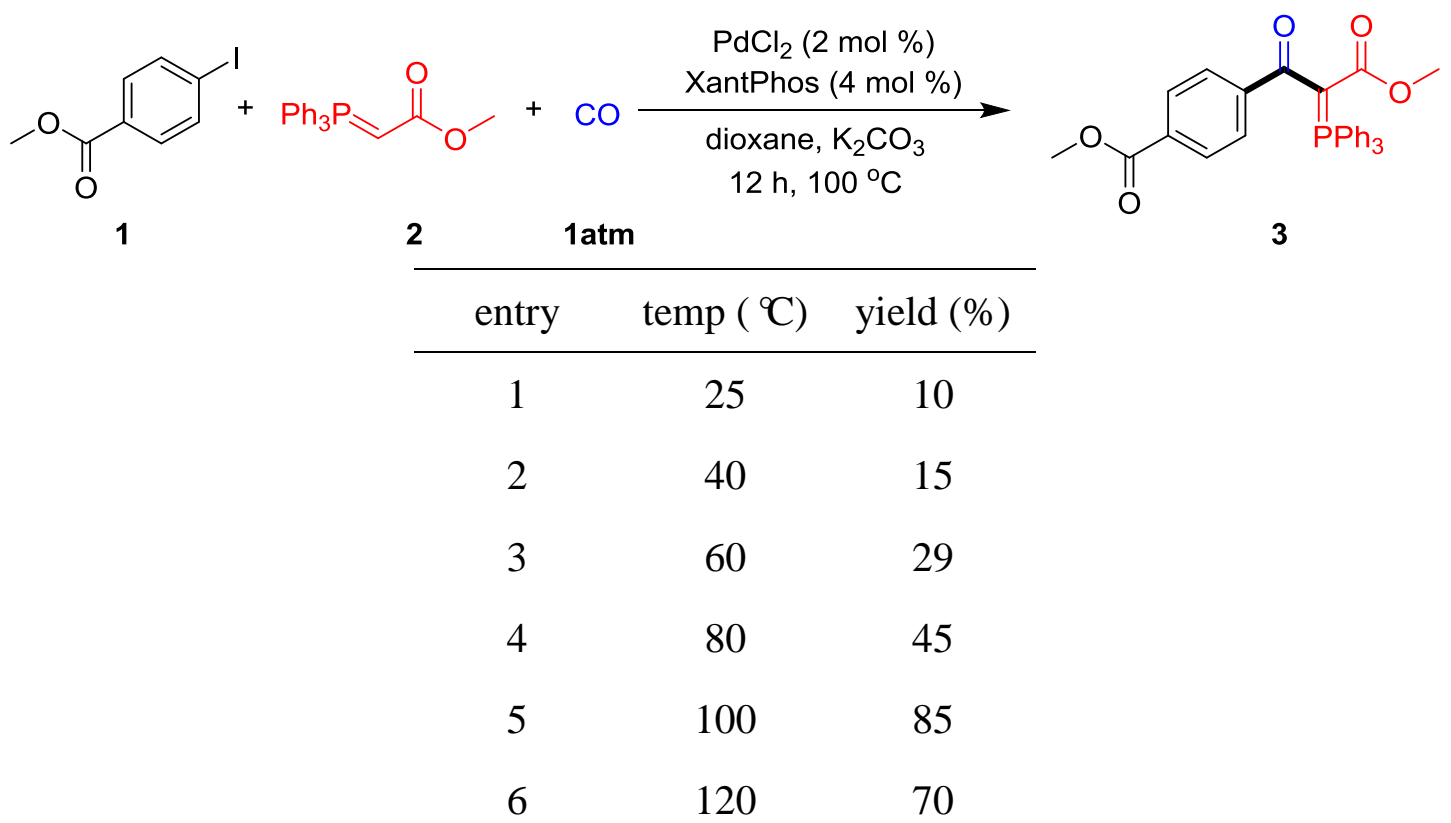

${ }^{\text {a }}$ Yield determined by ${ }^{1} \mathrm{H}$ NMR with 1, 3, 5-trimethoxybenzene as internal standard. 
Table S5: Screening the equivalent of the substrate. ${ }^{\text {a }}$

\begin{tabular}{|c|c|c|c|c|c|c|c|}
\hline O & & 2 & $\begin{array}{l}+\mathrm{CO}- \\
\mathbf{1} \mathbf{a t m}\end{array}$ & $\begin{array}{l}\mathrm{Cl}_{2}(2 \mathrm{~m} \\
\text { tPhos }(4 \\
\text { xane, } \mathrm{K}_{2} \\
2 \mathrm{~h}, 100\end{array}$ & $\begin{array}{l}\text { \%) } \\
\text { nol \%) } \\
O_{3} \\
C\end{array}$ & 0 & 3 \\
\hline entry & 1 (equiv) & 2 (equiv) & yield $(\%)$ & entry & 1 (equiv) & 2 (equiv) & yield (\%) \\
\hline 1 & 1.0 & 0.8 & 69 & 8 & 0.8 & 1.0 & 55 \\
\hline 2 & 1.0 & 1.0 & 85 & 9 & 1.0 & 1.0 & 85 \\
\hline 3 & 1.0 & 1.2 & 93 & 10 & 1.2 & 1.0 & 46 \\
\hline 4 & 1.0 & 1.4 & 88 & 11 & 1.4 & 1.0 & 45 \\
\hline 5 & 1.0 & 1.6 & 86 & 12 & 1.6 & 1.0 & 32 \\
\hline 6 & 1.0 & 1.8 & 94 & 13 & 1.8 & 1.0 & 52 \\
\hline 7 & 1.0 & 2.0 & 90 & 14 & 2.0 & 1.0 & 55 \\
\hline
\end{tabular}

${ }^{\mathrm{a}}$ Yield determined by ${ }^{1} \mathrm{H}$ NMR with 1, 3, 5-trimethoxybenzene as internal standard.

Table S6: Screening the reaction time. ${ }^{\mathrm{a}}$

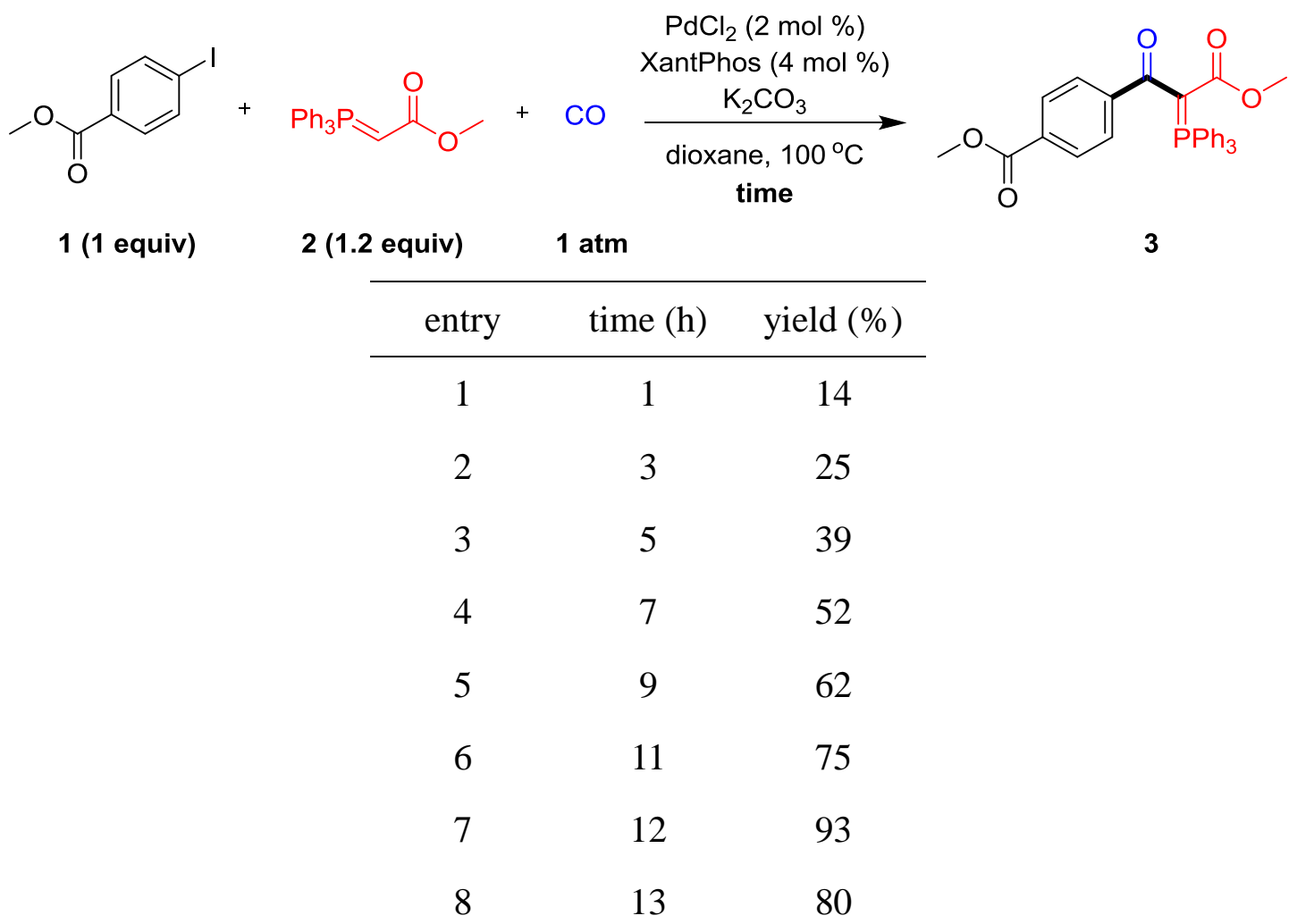

${ }^{\text {a }}$ Yield determined by ${ }^{1} \mathrm{H}$ NMR with 1, 3, 5-trimethoxybenzene as internal standard. 


\subsection{The optimization of reaction conditions for stabilized phosphorus ylides.}

\subsubsection{Typical reaction procedure for the optimization of reaction conditions.}

The reaction was carried out in an autoclave containing a $10 \mathrm{~mL}$ Teflon reaction tube. $\mathrm{PdCl}_{2}(0.01 \mathrm{mmol}, 2 \mathrm{~mol} \%)$, XantPhos $(0.02 \mathrm{mmol}, 4 \mathrm{~mol} \%)$ and a magnetic stir bar were placed in the tube, which was then capped with a stopper. Then, methyl $\begin{array}{llllll}\text { 4-iodobenzoate } & 1 & (0.5 & \mathrm{mmol}, & 1 & \text { equiv }) \text {, }\end{array}$ 1-phenyl-2-(triphenylphosphoranylidene)ethanone, $\mathrm{K}_{2} \mathrm{CO}_{3}(0.5 \mathrm{mmol}, 1$ equiv), and dioxane $(3 \mathrm{~mL})$ were added to the tube. The tube was placed in the autoclave. Once sealed, the autoclave was purged three times with $\mathrm{CO}$ at room temperature and then pressurized to $1 \mathrm{~atm}$ with $\mathrm{CO}$ at room temperature and heated in an oil bath at $100{ }^{\circ} \mathrm{C}$ for several hours. The autoclave was then cooled to room temperature and vented to discharge the excess $\mathrm{CO}$. The mixture was filtered by celite and concentrated in vacuo. The crude product was purified by column chromatography on silica gel using a mixture of ethyl acetate and petroleum ether as eluent to give the $\alpha$-acylphosphoranes.

Table S7: Screening the equivalents of the substrate. ${ }^{\mathrm{a}}$

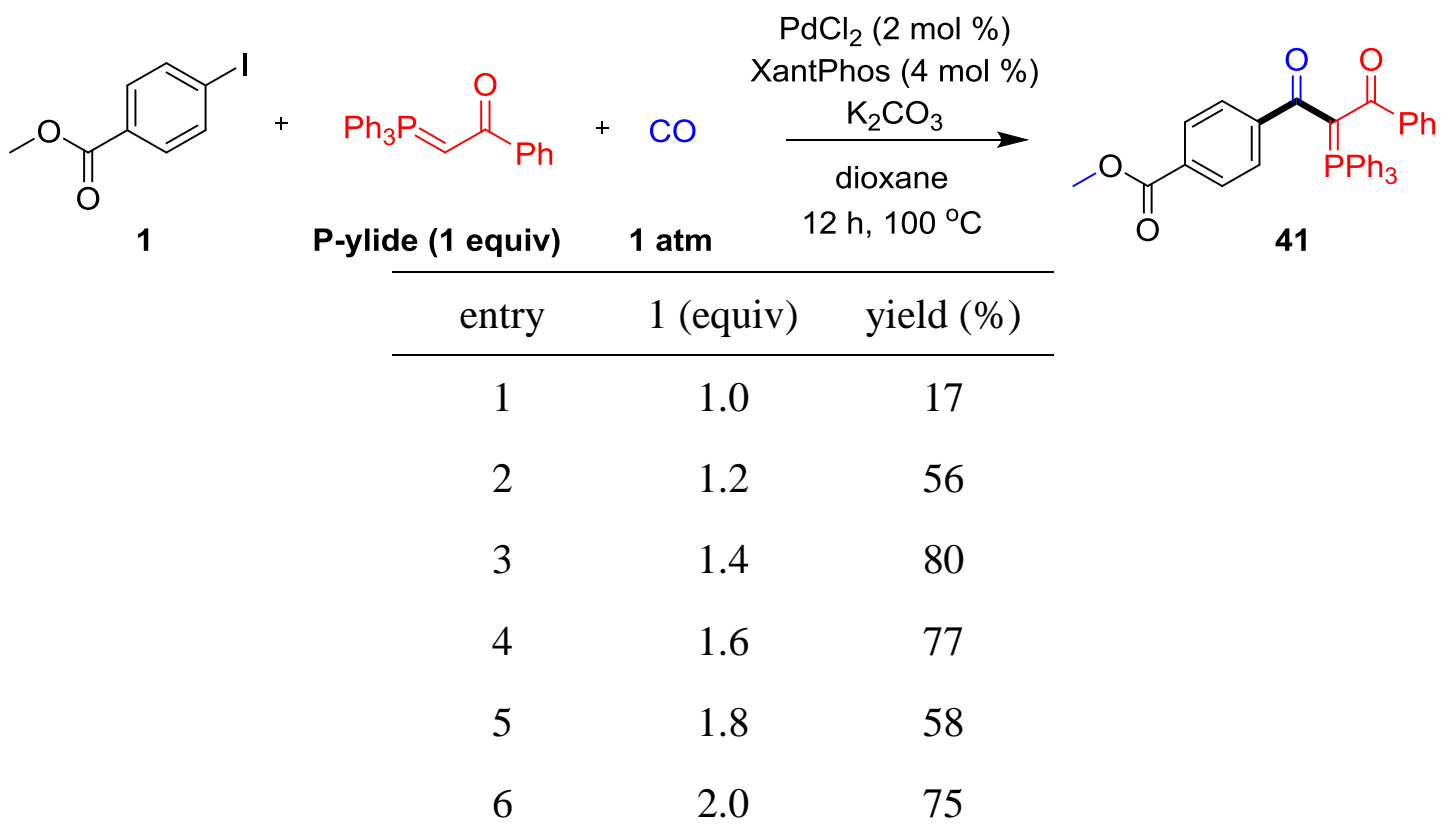

${ }^{\text {a }}$ Yield determined by ${ }^{1} \mathrm{H}$ NMR with 1, 3, 5-trimethoxybenzene as internal standard. 
Table S8: Screening the reaction time. ${ }^{\mathrm{a}}$

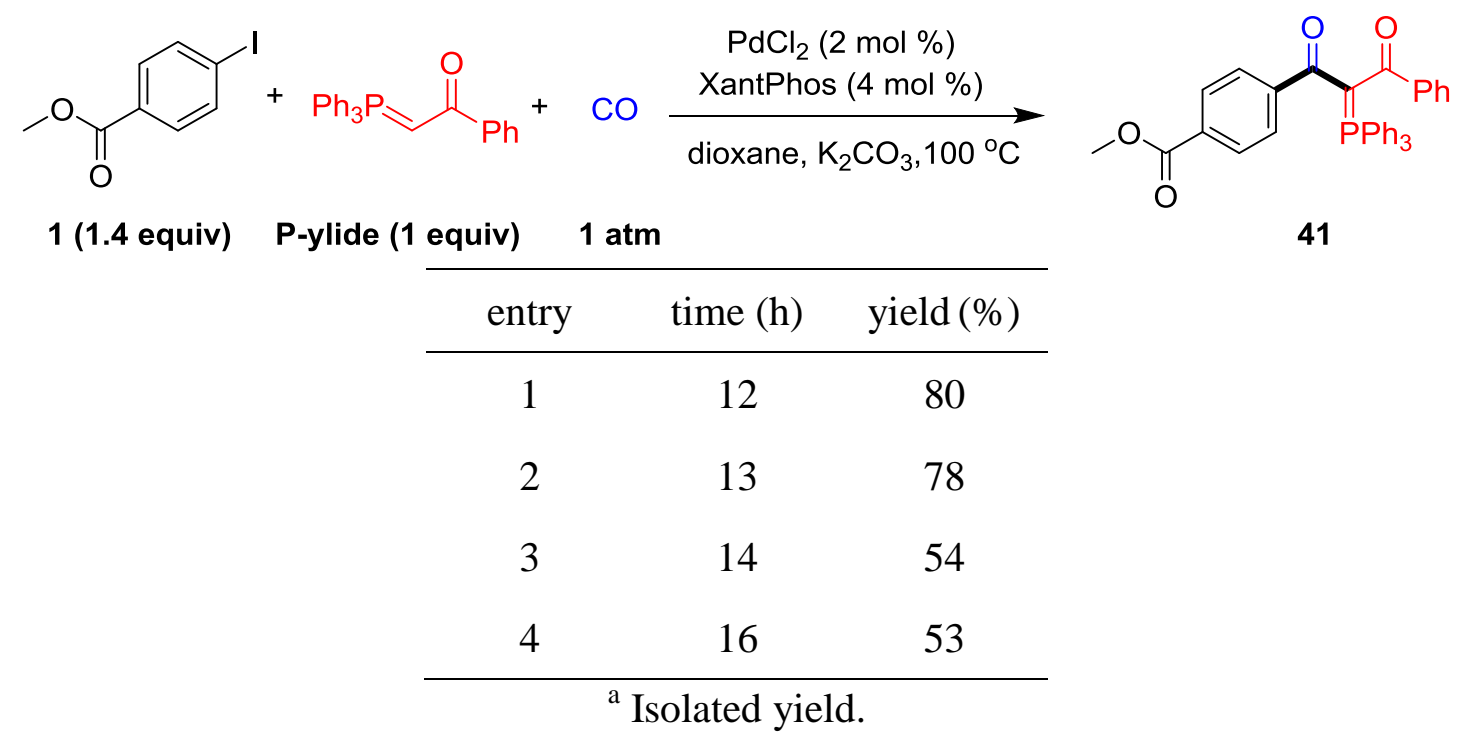

2.3 The optimization of reaction conditions for the synthesis of conjugated acetylenes.

\subsubsection{Typical reaction procedure for the optimization of reaction conditions.}

The reaction was carried out in a sealed tube containing $1 \mathrm{mmol}$ $\alpha$-acylphosphorane and a magnetic stir bar, which was subjected to microwave irradiation at appropriate temperature for several minutes.

Table S9: Screening the reaction temperature and time ${ }^{\mathrm{a}}$

\begin{tabular}{cccc}
\hline & \\
\hline entry & temp $\left({ }^{\circ} \mathrm{C}\right)$ & time $(\mathrm{min})$ & yield $(\%)$ \\
\hline 1 & 140 & 15 & 0 \\
2 & 180 & 15 & 0 \\
3 & 220 & 15 & 0 \\
4 & 260 & 15 & 0 \\
5 & 300 & 25 & 53 \\
6 & 300 & 30 & 42 \\
7 & 300 & 15 & 62 \\
\hline
\end{tabular}




\begin{tabular}{cccc}
\hline 8 & 300 & 10 & 48 \\
9 & 300 & 13 & 78 \\
\hline \multicolumn{3}{c}{${ }^{\text {a }}$ Isolated yield. }
\end{tabular}

\subsection{Typical procedure A for carbonylation of substituted aryl iodide with methyl}

\section{2-(triphenylphosphoranylidene)acetate 2.}

The reaction was carried out in an autoclave containing a $10 \mathrm{~mL}$ Teflon reaction tube. $\mathrm{PdCl}_{2}(0.01 \mathrm{mmol}, 2 \mathrm{~mol} \%)$, XantPhos (0.02 mmol, $\left.4 \mathrm{~mol} \%\right)$ and a magnetic stir bar were placed in the tube, which was then capped with a stopper. Then, substituted aryl iodide $(0.5$ mmol, 1 equiv), methyl 2-(triphenylphosphoranylidene)acetate 2 (0.6-1 mmol, 1.2-2 equiv), $\mathrm{K}_{2} \mathrm{CO}_{3}$ (0.5 mmol, 1 equiv), and dioxane ( $3 \mathrm{~mL}$ ) were added to the tube. The tube was placed in the autoclave. Once sealed, the autoclave was purged three times with $\mathrm{CO}$ at room temperature and then pressurized to $1 \mathrm{~atm}$ with $\mathrm{CO}$ at room temperature and heated in an oil bath at $100{ }^{\circ} \mathrm{C}$ for $12-24$ hours. The autoclave was then cooled to room temperature and vented to discharge the excess $\mathrm{CO}$. The mixture was filtered by celite and concentrated in vacuo. The crude product was purified by column chromatography on silica gel using a mixture of ethyl acetate and petroleum ether as eluent to give the $\alpha$-acylphosphoranes.

\subsection{Typical procedure B for carbonylation of several stabilized phosphonium ylides with methyl 4-iodobenzoate 1.}

The reaction was carried out in an autoclave containing a $10 \mathrm{~mL}$ Teflon reaction tube. $\mathrm{PdCl}_{2}(0.01 \mathrm{mmol}, 2 \mathrm{~mol} \%)$, XantPhos $(0.02 \mathrm{mmol}, 4 \mathrm{~mol} \%)$ and a magnetic stir bar were placed in the tube, which was then capped with a stopper. Then, the stabilized phosphonium ylides (0.5 mmol, 1 equiv), methyl 4-iodobenzoate 1 (0.7-1 mmol, 1.4-2 equiv), $\mathrm{K}_{2} \mathrm{CO}_{3}(0.5 \mathrm{mmol}, 1$ equiv), and dioxane $(3 \mathrm{~mL})$ were added to the tube. The tube was placed in the autoclave. Once sealed, the autoclave was purged three times with $\mathrm{CO}$ at room temperature and then pressurized to $1 \mathrm{~atm}$ with $\mathrm{CO}$ at room temperature and heated in an oil bath at $100{ }^{\circ} \mathrm{C}$ for $13-24$ hours. The autoclave was then cooled to room temperature and vented to discharge the excess $\mathrm{CO}$. The mixture was filtered by kieselguhr and concentrated in vacuo. The crude product was 
purified by column chromatography on silica gel using a mixture of ethyl acetate and petroleum ether as eluent to give the $\alpha$-acylphosphoranes.

\subsection{Typical procedure $\mathrm{C}$ for the synthesis of conjugated acetylenes.}

The reaction was carried out in a sealed tube $(10 \mathrm{~mL})$ containing $1 \mathrm{mmol}$ $\alpha$-acylphosphorane and a magnetic stir bar, which was subjected to microwave irradiation for $5-13$ minutes at $300{ }^{\circ} \mathrm{C}$, then the crude product was purified by column chromatography on silica gel using a mixture of ethyl acetate and petroleum ether as eluent to give the conjugated acetylenes.

\subsection{Typical procedure for carbonylation of gram-scale synthesis of 14 .}

The reaction was carried out in an autoclave containing a $200 \mathrm{~mL}$ Teflon reaction tube. $\mathrm{PdCl}_{2}(0.1 \mathrm{mmol})$, XantPhos $(0.2 \mathrm{mmol})$ and a magnetic stir bar were placed in the tube, which was then capped with a stopper. Then, iodobenzene (1.02 g, $5 \mathrm{mmol})$, methyl 2-(triphenylphosphoranylidene)acetate $(8 \mathrm{mmol}), \mathrm{K}_{2} \mathrm{CO}_{3}(5 \mathrm{mmol})$, and dioxane $(15 \mathrm{~mL})$ were added to the tube. The tube was placed in the autoclave. Once sealed, the autoclave was purged three times with $\mathrm{CO}$ at room temperature and then pressurized to $5 \mathrm{~atm}$ with $\mathrm{CO}$ at room temperature and heated in an oil bath at $100{ }^{\circ} \mathrm{C}$ for 24 hours. The autoclave was then cooled to room temperature and vented to discharge the excess CO. The mixture was filtered by celite and concentrated in vacuo. The crude product was purified by column chromatography on silica gel using a mixture of ethyl acetate and petroleum ether as eluent to give the $\alpha$-acylphosphoranes 12a $(1.75 \mathrm{~g}, 80 \%)$.

\section{Synthesis of the stabilized phosphorus ylides.}

The stabilized phosphonium ylides a-i were prepared according to the literature procedure. For an example of stabilized phosphonium a, the procedure for the preparation as follows: solution of 2-bromo-1-phenylethanone (2.39 g, 1 equiv) in methylene chloride $(10 \mathrm{~mL})$ was added to a solution of triphenylphosphine $(3.78 \mathrm{~g}$, 1.2 equiv) in methylene chloride $(10 \mathrm{~mL})$ under nitrogen. The mixture was stirred overnight and then added $100 \mathrm{~mL}$ ether. After stirred for $1 \mathrm{~h}$, the resulting phosphonium salt was filtered and the precipitate was washed with ether and dried 
under vacuum. The dried phosphonium salt was suspended in a mixture of water (50<smiles>O=C(C=Pc1ccccc1)c1ccccc1</smiles>

a<smiles>O=C(C=Pc1ccccc1)c1ccc(-c2ccccc2)cc1</smiles>

d<smiles>O=C(C=Pc1ccccc1)OCc1ccccc1</smiles>

g<smiles>Cc1ccc(C(=O)C=Pc2ccccc2)cc1</smiles>

b<smiles>O=C(C=Pc1ccccc1)c1ccc(C(F)(F)F)cc1</smiles>

$\mathbf{e}$<smiles>O=C(C=Pc1ccccc1)c1ccc2ccccc2c1</smiles>

h<smiles>COc1ccc(C(=O)/C=P/Pc2ccccc2)cc1</smiles>

c<smiles>N#Cc1ccc(C(=O)C=Pc2ccccc2)cc1</smiles>

f<smiles>N#CC=[Pb]</smiles>

i

$\mathrm{mL})$ and methanol $(50 \mathrm{~mL})$, and the mixture was stirred for $1 \mathrm{~h}$. Aqueous sodium hydroxide $(2 \mathrm{M})$ was added to the mixture until $\mathrm{pH}$ reached between 7 and 8 . The mixture was then stirred vigorously for $5 \mathrm{~h}$. After evaporated methanol, the aqueous layer was extracted with methylene chloride. Organic layer was dried over $\mathrm{Na}_{2} \mathrm{SO}_{4}$ and evaporated to obtain the 1-phenyl-2-(triphenylphosphoranylidene)ethanone (1.7 g, $75 \%$, a colorless solid). The structure was confirm by ${ }^{1} \mathrm{H}$ NMR spectra.

\begin{tabular}{|c|c|c|}
\hline entry & products & reference \\
\hline 1 & $\mathrm{a}$ & $\mathrm{S} 1$ \\
\hline 2 & $\mathrm{~b}$ & $\mathrm{~S} 2$ \\
\hline 3 & $\mathrm{c}$ & $\mathrm{S} 2$ \\
\hline 4 & $\mathrm{~d}$ & $\mathrm{~S} 3$ \\
\hline 5 & $\mathrm{e}$ & $\mathrm{S} 4$ \\
\hline 6 & $\mathrm{f}$ & $\mathrm{S} 2$ \\
\hline 7 & $\mathrm{~g}$ & $\mathrm{~S} 5$ \\
\hline 8 & $\mathrm{~h}$ & $\mathrm{~S} 2$ \\
\hline 9 & $\mathrm{i}$ & $\mathrm{S} 6$ \\
\hline
\end{tabular}




\section{References.}

S1. Venkateswararao, E.; Kim, M.-S.; Sharma, V. K.; Lee, K.-C.; Subramanian, S.; Roh, E.; Kim, Y.; Jung, S.-H. Eur. J. Med. Chem., 2013, 59, 31-38.

S2. Stark, D. G.; Morrill, L. C.; Yeh, P. P.; Slawin, A. M. Z.; O'Riordan, T. J. C.; Smith, A. D.; Angew. Chem Int. Ed., 2013, 52, 11642-11646.

S3. Karami, K.; Büyükgüngör, O.; Dalvand, H. Transition Met. Chem., 2010, 35, 621-626.

S4. Belmessieri, D.; Cordes, D. B.; Slawin, A. M.; Smith, A. D. Org. Lett., 2013, 15, 3472-3475.

S5. Tran, Y. S.; Kwon, O. Org. Lett., 2005, 7, 4289-4291.

S6. Burling, S.; Paine, B. M.; Nama, D.; Brown, V. S.; Mahon, M. F.; Prior, T. J.; Pregosin, P. S.; Whittlesey, M. K.; Williams, J. M. J. Am. Chem. Soc., 2007, 129, 1987-1995.

\section{Analytic data for products.}<smiles>COC(=O)c1ccc(C(=O)C(P)=Pc2ccccc2)cc1</smiles>

\section{Methyl 4-(3-methoxy-3-oxo-2-(triphenylphosphoranylidene)propanoyl)benzoate}

3. According to the general procedure A, methyl 4-iodobenzoate $(131.0 \mathrm{mg}, 0.5$ mmol), methyl 2-(triphenylphosphoranylidene)acetate (200.6 mg, $0.6 \mathrm{mmol}$ ),

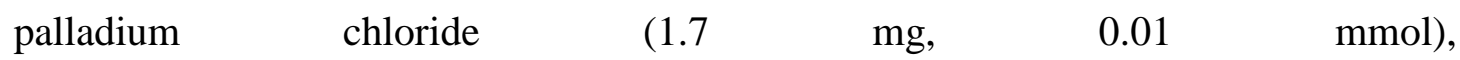
(9,9-dimethyl-9H-xanthene-4,5-diyl)bis(diphenylphosphine) (11.5 mg, $0.02 \mathrm{mmol}$ ), Potassium carbonate $(69.1 \mathrm{mg}, 0.5 \mathrm{mmol}), 1$,4-dioxane $(3 \mathrm{~mL}), \mathrm{CO}(1 \mathrm{~atm}), 100{ }^{\circ} \mathrm{C}$, $12 \mathrm{~h}$, compound 3 was synthesized and purified on silica gel with EtOAc: PE (V:V = $\left.1: 2, \mathrm{R}_{\mathrm{f}}=0.15\right)$ to give a pale yellow solid $(218 \mathrm{mg}, 88 \%), \mathrm{mp}=206-208{ }^{\circ} \mathrm{C} ;{ }^{1} \mathrm{H}$ NMR (600 MHz, $\left.\mathrm{CDCl}_{3}\right), \delta(\mathrm{ppm})=8.02(\mathrm{~d}, J=8.2 \mathrm{~Hz}, 2 \mathrm{H})$, 7.77-7.74 (m, $\left.6 \mathrm{H}\right)$, $7.68(\mathrm{~d}, J=8.2 \mathrm{~Hz}, 2 \mathrm{H}), 7.57-7.54(\mathrm{~m}, 3 \mathrm{H}), 7.50-7.47(\mathrm{~m}, 6 \mathrm{H}), 3.90(\mathrm{~s}, 3 \mathrm{H}), 3.12$ (s, $3 \mathrm{H}) ;{ }^{13} \mathrm{C}$ NMR $\left(150 \mathrm{MHz}, \mathrm{CDCl}_{3}\right), \delta(\mathrm{ppm})=192.3\left(\mathrm{~d}, J_{\mathrm{P}-\mathrm{C}}=5.4 \mathrm{~Hz}\right), 167.9\left(\mathrm{~d}, J_{\mathrm{P}-\mathrm{C}}\right.$ $=14.3 \mathrm{~Hz}), 167.0,147.7\left(\mathrm{~d}, J_{\mathrm{P}-\mathrm{C}}=9.4 \mathrm{~Hz}\right), 133.4\left(\mathrm{~d}, J_{\mathrm{P}-\mathrm{C}}=9.8 \mathrm{~Hz}\right), 132.1\left(\mathrm{~d}, J_{\mathrm{P}-\mathrm{C}}=\right.$ $2.9 \mathrm{~Hz}), 130.4,128.8,128.7\left(\mathrm{~d}, J_{\mathrm{P}-\mathrm{C}}=12.3 \mathrm{~Hz}\right), 127.8,125.7\left(\mathrm{~d}, J_{\mathrm{P}-\mathrm{C}}=92.9 \mathrm{~Hz}\right)$, $70.1\left(\mathrm{~d}, J_{\mathrm{P}-\mathrm{C}}=111.0 \mathrm{~Hz}\right), 52.0,49.8 ;{ }^{31} \mathrm{P} \mathrm{NMR}\left(162 \mathrm{MHz}, \mathrm{CDCl}_{3}\right), \delta(\mathrm{ppm})=18.7$; HRMS (ESI) Calcd for $\mathrm{C}_{30} \mathrm{H}_{25} \mathrm{O}_{5} \mathrm{P}[\mathrm{M}+\mathrm{H}]^{+}=497.1512$, Found $=497.1515$. 


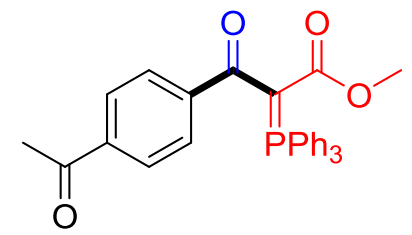

Methyl 3-(4-acetylphenyl)-3-oxo-2-(triphenylphosphoranylidene)propanoate 4.

According to the general procedure A, 1-(4-iodophenyl)ethanone (123.0 mg, 0.5 mmol), methyl 2-(triphenylphosphoranylidene)acetate $(200.6 \mathrm{mg}, 0.6 \mathrm{mmol})$, palladium chloride $\quad(1.7 \quad \mathrm{mg}, \quad 0.01 \quad \mathrm{mmol})$, (9,9-dimethyl-9H-xanthene-4,5-diyl)bis(diphenylphosphine) (11.5 mg, $0.02 \mathrm{mmol}$ ), Potassium carbonate (69.1 mg, $0.5 \mathrm{mmol}), 1,4$-dioxane $(3 \mathrm{~mL}), \mathrm{CO}(1 \mathrm{~atm}), 100{ }^{\circ} \mathrm{C}$, $12 \mathrm{~h}$, compound 4 was synthesized and purified on silica gel with EtOAc:PE (V:V = $\left.1: 2, \mathrm{R}_{\mathrm{f}}=0.10\right)$ to give a pale yellow solid $(226 \mathrm{mg}, 94 \%), \mathrm{mp}=178-180{ }^{\circ} \mathrm{C} ;{ }^{1} \mathrm{H}$ NMR $\left(400 \mathrm{MHz}, \mathrm{CDCl}_{3}\right), \delta(\mathrm{ppm})=7.94(\mathrm{~d}, J=8.3 \mathrm{~Hz}, 2 \mathrm{H}), 7.78-7.69(\mathrm{~m}, 8 \mathrm{H})$, 7.59-7.55 (m, $3 \mathrm{H}), 7.52-7.47(\mathrm{~m}, 6 \mathrm{H}), 3.11(\mathrm{~s}, 3 \mathrm{H}), 2.60(\mathrm{~s}, 3 \mathrm{H}) ;{ }^{13} \mathrm{C} \mathrm{NMR}(100$ $\left.\mathrm{MHz}, \mathrm{CDCl}_{3}\right), \delta(\mathrm{ppm})=198.0,192.3\left(\mathrm{~d}, J_{\mathrm{P}-\mathrm{C}}=5.5 \mathrm{~Hz}\right), 167.9\left(\mathrm{~d}, J_{\mathrm{P}-\mathrm{C}}=14.1 \mathrm{~Hz}\right)$, $147.7\left(\mathrm{~d}, J_{\text {P-C }}=9.0 \mathrm{~Hz}\right), 137.3,133.4\left(\mathrm{~d}, J_{\text {P-C }}=9.9 \mathrm{~Hz}\right), 132.1\left(\mathrm{~d}, J_{\text {P-C }}=2.9 \mathrm{~Hz}\right)$, $128.7\left(\mathrm{~d}, J_{\text {P-C }}=12.5 \mathrm{~Hz}\right), 128.0,127.6,125.7\left(\mathrm{~d}, J_{\text {P-C }}=92.9 \mathrm{~Hz}\right), 70.1\left(\mathrm{~d}, J_{\text {P-C }}=\right.$ 110.3 Hz), 49.8, 26.8; ${ }^{31} \mathrm{P} \mathrm{NMR}\left(162 \mathrm{MHz}, \mathrm{CDCl}_{3}\right), \delta(\mathrm{ppm})=18.7$; HRMS (ESI) Calcd for $\mathrm{C}_{30} \mathrm{H}_{25} \mathrm{O}_{4} \mathrm{P}[\mathrm{M}+\mathrm{H}]^{+}=481.1563$, Found $=481.1562$.<smiles>COC(=O)C(=P)C(=O)c1ccc(F)cc1</smiles>

Methyl 3-(4-fluorophenyl)-3-oxo-2-(triphenylphosphoranylidene)propanoate 5.

According to the general procedure A, 1-fluoro-4-iodobenzene (111.0 mg, $0.5 \mathrm{mmol})$, methyl 2-(triphenylphosphoranylidene)acetate (200.6 mg, $0.6 \mathrm{mmol})$, palladium chloride

$\mathrm{mg}$ 0.01 $\mathrm{mmol})$, (9,9-dimethyl-9H-xanthene-4,5-diyl)bis(diphenylphosphine) (11.5 mg, $0.02 \mathrm{mmol}$ ), Potassium carbonate (69.1 mg, $0.5 \mathrm{mmol}), 1$,4-dioxane $(3 \mathrm{~mL}), \mathrm{CO}(1 \mathrm{~atm}), 100{ }^{\circ} \mathrm{C}$, $12 \mathrm{~h}$, compound 5 was synthesized and purified on silica gel with EtOAc:PE (V:V = $\left.1: 2, \mathrm{R}_{\mathrm{f}}=0.24\right)$ to give a yellow solid $(176 \mathrm{mg}, 77 \%), \mathrm{mp}=154-158{ }^{\circ} \mathrm{C} ;{ }^{1} \mathrm{H} \mathrm{NMR}$ $\left(400 \mathrm{MHz}, \mathrm{CDCl}_{3}\right), \delta(\mathrm{ppm})=7.79-7.71(\mathrm{~m}, 8 \mathrm{H}), 7.57-7.53(\mathrm{~m}, 3 \mathrm{H}), 7.50-7.46(\mathrm{~m}, 6$ 
$\mathrm{H}), 7.02(\mathrm{t}, J=8.7 \mathrm{~Hz}, 2 \mathrm{H}), 3.16(\mathrm{~s}, 3 \mathrm{H}),{ }^{13} \mathrm{C} \mathrm{NMR}\left(100 \mathrm{MHz}, \mathrm{CDCl}_{3}\right), \delta(\mathrm{ppm})=$ $191.9\left(\mathrm{~d}, J_{\mathrm{P}-\mathrm{C}}=5.4 \mathrm{~Hz}\right), 167.9\left(\mathrm{~d}, J_{\mathrm{P}-\mathrm{C}}=14.1 \mathrm{~Hz}\right), 163.7\left(\mathrm{~d}, J_{\mathrm{F}-\mathrm{C}}=246.8 \mathrm{~Hz}\right), 139.0$ $\left(\mathrm{q}, J_{\mathrm{F}-\mathrm{C}}=3.13 \mathrm{~Hz}\right), 133.3\left(\mathrm{~d}, J_{\mathrm{P}-\mathrm{C}}=9.7 \mathrm{~Hz}\right), 132.0\left(\mathrm{~d}, J_{\mathrm{P}-\mathrm{C}}=2.8 \mathrm{~Hz}\right), 130.6\left(\mathrm{~d}, J_{\mathrm{F}-\mathrm{C}}\right.$ $=8.5 \mathrm{~Hz}), 128.7\left(\mathrm{~d}, J_{\mathrm{P}-\mathrm{C}}=12.5 \mathrm{~Hz}\right), 126.5\left(\mathrm{~d}, J_{\mathrm{P}-\mathrm{C}}=92.7 \mathrm{~Hz}\right), 114.1\left(\mathrm{~d}, J_{\text {F-C }}=21.5\right.$ $\mathrm{Hz}), 69.2\left(\mathrm{~d}, J_{\mathrm{P}-\mathrm{C}}=110.7 \mathrm{~Hz}\right), 49.7 ;{ }^{31} \mathrm{P} \mathrm{NMR}\left(162 \mathrm{MHz}, \mathrm{CDCl}_{3}\right), \delta(\mathrm{ppm})=19.2$; HRMS (ESI) Calcd for $\mathrm{C}_{28} \mathrm{H}_{22} \mathrm{FO}_{3} \mathrm{P}[\mathrm{M}+\mathrm{H}]^{+}=457.1363$, Found $=457.1362$.<smiles>COC(=O)C(=P)C(=O)c1ccc(Cl)cc1</smiles>

Methyl 3-(4-chlorophenyl)-3-oxo-2-(triphenylphosphoranylidene)propanoate 6. According to the general procedure A, 1-chloro-4-iodobenzene (119.2 mg, $0.5 \mathrm{mmol})$, methyl 2-(triphenylphosphoranylidene)acetate (267.4 mg, $0.8 \mathrm{mmol})$, palladium chloride

$\mathrm{mg}$,

0.01

mmol), (9,9-dimethyl-9H-xanthene-4,5-diyl)bis(diphenylphosphine) (11.5 mg, $0.02 \mathrm{mmol}$ ), Potassium carbonate (69.1 mg, $0.5 \mathrm{mmol}), 1$,4-dioxane $(3 \mathrm{~mL}), \mathrm{CO}(1 \mathrm{~atm}), 100{ }^{\circ} \mathrm{C}$, $12 \mathrm{~h}$, compound 6 was synthesized and purified on silica gel with EtOAc:PE (V:V = $\left.1: 2, \mathrm{R}_{\mathrm{f}}=0.35\right)$ to give a colorless solid $(177 \mathrm{mg}, 75 \%), \mathrm{mp}=143-145{ }^{\circ} \mathrm{C} ;{ }^{1} \mathrm{H} \mathrm{NMR}$ $\left(400 \mathrm{MHz}, \mathrm{CDCl}_{3}\right), \delta(\mathrm{ppm})=7.77-7.72(\mathrm{~m}, 6 \mathrm{H}), 7.63(\mathrm{~d}, J=8.4 \mathrm{~Hz}, 2 \mathrm{H}), 7.57-7.54$ (m, $3 \mathrm{H}), 7.50-7.46(\mathrm{~m}, 6 \mathrm{H}), 7.31(\mathrm{~d}, J=8.4 \mathrm{~Hz}, 2 \mathrm{H}), 3.15(\mathrm{~s}, 3 \mathrm{H}),{ }^{13} \mathrm{C}$ NMR $(100$ $\left.\mathrm{MHz} \mathrm{CDCl}_{3}\right), \delta(\mathrm{ppm})=191.9\left(\mathrm{~d}, J_{\mathrm{P}-\mathrm{C}}=5.5 \mathrm{~Hz}\right), 167.9\left(\mathrm{~d}, J_{\mathrm{P}-\mathrm{C}}=14.3 \mathrm{~Hz}\right), 141.3(\mathrm{~d}$, $\left.J_{\text {P-C }}=9.1 \mathrm{~Hz}\right), 135.4,133.3\left(\mathrm{~d}, J_{\mathrm{P}-\mathrm{C}}=9.9 \mathrm{~Hz}\right), 132.0\left(\mathrm{~d}, J_{\mathrm{P}-\mathrm{C}}=2.9 \mathrm{~Hz}\right), 129.8,128.7$ $\left(\mathrm{d}, J_{\mathrm{P}-\mathrm{C}}=12.6 \mathrm{~Hz}\right), 127.5,125.9\left(\mathrm{~d}, J_{\mathrm{P}-\mathrm{C}}=92.7 \mathrm{~Hz}\right), 69.4\left(\mathrm{~d}, J_{\mathrm{P}-\mathrm{C}}=110.6 \mathrm{~Hz}\right), 49.8$; ${ }^{31} \mathrm{P}$ NMR $\left(162 \mathrm{MHz}, \mathrm{CDCl}_{3}\right), \delta(\mathrm{ppm})=19.0$; HRMS (ESI) Calcd for $\mathrm{C}_{28} \mathrm{H}_{22} \mathrm{ClO}_{3} \mathrm{P}$ $[\mathrm{M}+\mathrm{H}]^{+}=473.1068$, Found $=473.1058$.<smiles>COC(=O)C(=P)C(=O)c1ccc(Br)cc1</smiles>

Methyl 3-(4-bromophenyl)-3-oxo-2-(triphenylphosphoranylidene)propanoate 7. According to the general procedure A, 1-bromo-4-iodobenzene (141.4 mg, $0.5 \mathrm{mmol}$ ), methyl 2-(triphenylphosphoranylidene)acetate (200.6 mg, $0.6 \mathrm{mmol})$, palladium 
chloride

$\mathrm{mg}$,

0.01

mmol),

(9,9-dimethyl-9H-xanthene-4,5-diyl)bis(diphenylphosphine) (11.5 mg, $0.02 \mathrm{mmol}$ ), Potassium carbonate (69.1 mg, $0.5 \mathrm{mmol}), 1$,4-dioxane $(3 \mathrm{~mL}), \mathrm{CO}(1 \mathrm{~atm}), 100{ }^{\circ} \mathrm{C}$, $12 \mathrm{~h}$, compound 7 was synthesized and purified on silica gel with EtOAc:PE $(\mathrm{V}: \mathrm{V}=$ $\left.1: 2, \mathrm{R}_{\mathrm{f}}=0.32\right)$ to give a pale yellow solid $(237 \mathrm{mg}, 92 \%), \mathrm{mp}=150-153{ }^{\circ} \mathrm{C} ;{ }^{1} \mathrm{H}$ NMR $\left(600 \mathrm{MHz}, \mathrm{CDCl}_{3}\right), \delta(\mathrm{ppm})=7.76-7.72(\mathrm{~m}, 6 \mathrm{H}), 7.57-7.55(\mathrm{~m}, 5 \mathrm{H})$, 7.50-7.46 (m, $8 \mathrm{H}), 3.14(\mathrm{~s}, 3 \mathrm{H}) ;{ }^{13} \mathrm{C} \mathrm{NMR}\left(100 \mathrm{MHz}, \mathrm{CDCl}_{3}\right), \delta(\mathrm{ppm})=191.9(\mathrm{~d}, J$ P-C $=5.4 \mathrm{~Hz}), 167.8\left(\mathrm{~d}, J_{\mathrm{P}-\mathrm{C}}=14.0 \mathrm{~Hz}\right), 141.8\left(\mathrm{~d}, J_{\mathrm{P}-\mathrm{C}}=9.2 \mathrm{~Hz}\right), 133.3\left(\mathrm{~d}, J_{\mathrm{P}-\mathrm{C}}=9.7\right.$ $\mathrm{Hz}), 132.0\left(\mathrm{~d}, J_{\mathrm{P}-\mathrm{C}}=2.8 \mathrm{~Hz}\right), 130.4,130.0,128.6\left(\mathrm{~d}, J_{\mathrm{P}-\mathrm{C}}=12.5 \mathrm{~Hz}\right), 125.8\left(\mathrm{~d}, J_{\mathrm{P}-\mathrm{C}}\right.$ $=92.8 \mathrm{~Hz}), 123.8,69.4\left(\mathrm{~d}, J_{\mathrm{P}-\mathrm{C}}=110.5 \mathrm{~Hz}\right), 49.7 ;{ }^{31} \mathrm{P} \mathrm{NMR}\left(162 \mathrm{MHz}, \mathrm{CDCl}_{3}\right), \delta$ $(\mathrm{ppm})=19.0 ; \mathrm{HRMS}(\mathrm{ESI})$ Calcd for $\mathrm{C}_{28} \mathrm{H}_{22} \mathrm{BrO}_{3} \mathrm{P}[\mathrm{M}+\mathrm{H}]^{+}=517.0563$, Found $=$ 517.0557.<smiles>COC(=O)C(=P)C(=O)c1ccc([N+](=O)[O-])cc1</smiles>

Methyl 3-(4-nitrophenyl)-3-oxo-2-(triphenylphosphoranylidene)propanoate 8.

According to the general procedure A, 1-iodo-4-nitrobenzene (124.5 mg, $0.5 \mathrm{mmol})$, methyl 2-(triphenylphosphoranylidene)acetate $(267.4 \mathrm{mg}, 0.8 \mathrm{mmol})$, palladium chloride

$\mathrm{mg}$, 0.01 mmol), (9,9-dimethyl-9H-xanthene-4,5-diyl)bis(diphenylphosphine) (11.5 mg, $0.02 \mathrm{mmol}$ ), Potassium carbonate $(69.1 \mathrm{mg}, 0.5 \mathrm{mmol}), 1$,4-dioxane $(3 \mathrm{~mL}), \mathrm{CO}(1 \mathrm{~atm}), 100{ }^{\circ} \mathrm{C}$, $12 \mathrm{~h}$, compound $\mathbf{8}$ was synthesized and purified on silica gel with EtOAc:PE (V:V = $\left.1: 2, \mathrm{R}_{\mathrm{f}}=0.21\right)$ to give a yellow solid $(193 \mathrm{mg}, 80 \%), \mathrm{mp}=193-196{ }^{\circ} \mathrm{C} ;{ }^{1} \mathrm{H} \mathrm{NMR}$ $\left(400 \mathrm{MHz}, \mathrm{CDCl}_{3}\right), \delta(\mathrm{ppm})=8.20(\mathrm{~d}, J=8.7 \mathrm{~Hz}, 2 \mathrm{H}), 7.78-7.73(\mathrm{~m}, 8 \mathrm{H}), 7.61-7.49$ $(\mathrm{m}, 9 \mathrm{H}), 3.11(\mathrm{~s}, 3 \mathrm{H}) ;{ }^{13} \mathrm{C} \mathrm{NMR}\left(100 \mathrm{MHz}, \mathrm{CDCl}_{3}\right), \delta(\mathrm{ppm})=191.0\left(\mathrm{~d}, J_{\mathrm{P}-\mathrm{C}}=5.8\right.$ $\mathrm{Hz}), 167.7\left(\mathrm{~d}, J_{\text {P-C }}=13.8 \mathrm{~Hz}\right), 149.5\left(\mathrm{~d}, J_{\text {P-C }}=9.4 \mathrm{~Hz}\right), 148.0,133.4\left(\mathrm{~d}, J_{\text {P-C }}=9.8\right.$ $\mathrm{Hz}), 132.2\left(\mathrm{~d}, J_{\text {P-C }}=2.9 \mathrm{~Hz}\right), 128.8\left(\mathrm{~d}, J_{\mathrm{P}-\mathrm{C}}=12.5 \mathrm{~Hz}\right), 128.6,125.3\left(\mathrm{~d}, J_{\text {P-C }}=92.9\right.$ $\mathrm{Hz}), 122.8,70.6\left(\mathrm{~d}, J_{\mathrm{P}-\mathrm{C}}=109.9 \mathrm{~Hz}\right), 49.9 ;{ }^{31} \mathrm{P} \mathrm{NMR}\left(162 \mathrm{MHz}, \mathrm{CDCl}_{3}\right), \delta(\mathrm{ppm})=$ 18.6; HRMS (ESI) Calcd for $\mathrm{C}_{28} \mathrm{H}_{22} \mathrm{NO}_{5} \mathrm{P}[\mathrm{M}+\mathrm{H}]^{+}=484.1308$, Found $=484.1306$. 
<smiles>COC(=O)C(=P)C(=O)c1ccc(C(F)(F)F)cc1</smiles>

Methyl-3-oxo-3-(4-(trifluoromethyl)phenyl)-2-(triphenylphosphoranylidene)prop

-anoate 9. According to the general procedure A, 1-iodo-4-(trifluoromethyl)benzene (136.0 mg, $0.5 \mathrm{mmol}$ ), methyl 2-(triphenylphosphoranylidene)acetate (200.6 mg, 0.6 mmol), palladium chloride $(1.7 \quad \mathrm{mg}, \quad 0.01 \quad \mathrm{mmol})$, (9,9-dimethyl-9H-xanthene-4,5-diyl)bis(diphenylphosphine) (11.5 mg, $0.02 \mathrm{mmol}$ ), Potassium carbonate (69.1 mg, $0.5 \mathrm{mmol}), 1$,4-dioxane $(3 \mathrm{~mL}), \mathrm{CO}(1 \mathrm{~atm}), 100{ }^{\circ} \mathrm{C}$, $12 \mathrm{~h}$, compound 9 was synthesized and purified on silica gel with EtOAc:PE $(\mathrm{V}: \mathrm{V}=$ $\left.1: 2, \mathrm{R}_{\mathrm{f}}=0.25\right)$ to give a colorless solid $(238 \mathrm{mg}, 94 \%), \mathrm{mp}=136-139{ }^{\circ} \mathrm{C} ;{ }^{1} \mathrm{H} \mathrm{NMR}$ $\left(400 \mathrm{MHz}, \mathrm{CDCl}_{3}\right), \delta(\mathrm{ppm})=7.78-7.73(\mathrm{~m}, 8 \mathrm{H}), 7.60-7.55(\mathrm{~m}, 5 \mathrm{H}), 7.52-7.47(\mathrm{~m}, 6$ $\mathrm{H}), 3.12(\mathrm{~s}, 3 \mathrm{H}) ;{ }^{13} \mathrm{C} \mathrm{NMR}\left(100 \mathrm{MHz}, \mathrm{CDCl}_{3}\right), \delta(\mathrm{ppm})=191.8\left(\mathrm{~d}, J_{\mathrm{P}-\mathrm{C}}=5.5 \mathrm{~Hz}\right)$, $167.8\left(\mathrm{~d}, J_{\mathrm{P}-\mathrm{C}}=14.0 \mathrm{~Hz}\right), 146.5\left(\mathrm{~d}, J_{\mathrm{P}-\mathrm{C}}=8.7 \mathrm{~Hz}\right), 133.4\left(\mathrm{~d}, J_{\mathrm{P}-\mathrm{C}}=9.7 \mathrm{~Hz}\right), 132.1(\mathrm{~d}$, $\left.J_{\text {P-C }}=2.9 \mathrm{~Hz}\right), 130.9\left(\mathrm{~d}, J_{\text {F-C }}=32.0 \mathrm{~Hz}\right), 128.7\left(\mathrm{~d}, J_{\mathrm{P}-\mathrm{C}}=12.5 \mathrm{~Hz}\right), 128.3,125.7(\mathrm{~d}, J$ P-C $=92.9 \mathrm{~Hz}), 124.3\left(\mathrm{q}, J_{\mathrm{F}-\mathrm{C}}=3.8 \mathrm{~Hz}\right), 124.2\left(\mathrm{~d}, J_{\mathrm{F}-\mathrm{C}}=270.4 \mathrm{~Hz}\right), 70.0\left(\mathrm{~d}, J_{\text {P-C }}=\right.$ 110.2 Hz), 49.8; ${ }^{31} \mathrm{P}$ NMR (162 MHz, $\left.\mathrm{CDCl}_{3}\right), \delta(\mathrm{ppm})=18.8$; HRMS (ESI) Calcd for $\mathrm{C}_{29} \mathrm{H}_{22} \mathrm{~F}_{3} \mathrm{O}_{3} \mathrm{P}[\mathrm{M}+\mathrm{H}]^{+}=507.1331$, Found $=507.1326$.<smiles>COC(=O)C(=P)C(=O)c1ccc(C)cc1</smiles>

Methyl 3-oxo-3-(p-tolyl)-2-(triphenylphosphoranylidene)propanoate

10.

According to the general procedure A, 1-iodo-4-methylbenzene (109.0 mg, $0.5 \mathrm{mmol})$, methyl 2-(triphenylphosphoranylidene)acetate $(267.4 \mathrm{mg}, 0.8 \mathrm{mmol})$, palladium chloride

$\mathrm{mg}$, 0.01 mmol), (9,9-dimethyl-9H-xanthene-4,5-diyl)bis(diphenylphosphine) (11.5 mg, $0.02 \mathrm{mmol}$ ), Potassium carbonate (69.1 mg, $0.5 \mathrm{mmol}), 1$,4-dioxane $(3 \mathrm{~mL}), \mathrm{CO}(1 \mathrm{~atm}), 100{ }^{\circ} \mathrm{C}$, $24 \mathrm{~h}$, compound 10 was synthesized and purified on silica gel with EtOAc:PE (V:V = $\left.1: 2, \mathrm{R}_{\mathrm{f}}=0.16\right)$ to give a pale yellow solid $(147 \mathrm{mg}, 65 \%), \mathrm{mp}=173-176{ }^{\circ} \mathrm{C} ;{ }^{1} \mathrm{H}$ NMR (400 MHz, $\left.\mathrm{CDCl}_{3}\right), \delta(\mathrm{ppm})=7.79-7.73(\mathrm{~m}, 6 \mathrm{H}), 7.62(\mathrm{~d}, J=8.0 \mathrm{~Hz}, 2 \mathrm{H})$, 
7.55-7.45 (m, $9 \mathrm{H}), 7.15(\mathrm{~d}, J=7.9 \mathrm{~Hz}, 2 \mathrm{H}), 3.15(\mathrm{~s}, 3 \mathrm{H}), 2.36(\mathrm{~s}, 3 \mathrm{H}) ;{ }^{13} \mathrm{C} \mathrm{NMR}$ $\left(100 \mathrm{MHz}, \mathrm{CDCl}_{3}\right), \delta(\mathrm{ppm})=193.2\left(\mathrm{~d}, J_{\mathrm{P}-\mathrm{C}}=5.2 \mathrm{~Hz}\right), 168.1\left(\mathrm{~d}, J_{\mathrm{P}-\mathrm{C}}=14.3 \mathrm{~Hz}\right)$, $140.0\left(\mathrm{~d}, J_{\mathrm{P}-\mathrm{C}}=8.9 \mathrm{~Hz}\right), 139.7,133.4\left(\mathrm{~d}, J_{\mathrm{P}-\mathrm{C}}=9.9 \mathrm{~Hz}\right), 131.8\left(\mathrm{~d}, J_{\mathrm{P}-\mathrm{C}}=2.9 \mathrm{~Hz}\right)$, $128.6\left(\mathrm{~d}, J_{\mathrm{P}-\mathrm{C}}=12.6 \mathrm{~Hz}\right), 128.0,126.3\left(\mathrm{~d}, J_{\mathrm{P}-\mathrm{C}}=92.7 \mathrm{~Hz}\right), 68.8\left(\mathrm{~d}, J_{\mathrm{P}-\mathrm{C}}=110.9 \mathrm{~Hz}\right)$, 49.7, 21.5; ${ }^{31} \mathrm{P}$ NMR (162 MHz, $\left.\mathrm{CDCl}_{3}\right), \delta(\mathrm{ppm})=19.1$; HRMS (ESI) Calcd for $\mathrm{C}_{29} \mathrm{H}_{25} \mathrm{O}_{3} \mathrm{P}[\mathrm{M}+\mathrm{H}]^{+}=453.1614$, Found $=453.1609$.

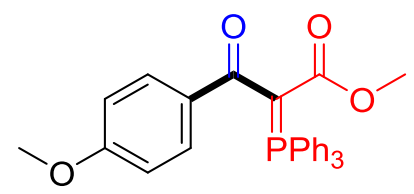

Methyl 3-(4-methoxyphenyl)-3-oxo-2-(triphenylphosphoranylidene)propanoate

11. According to the general procedure A, 1-iodo-4-methoxybenzene $(117.0 \mathrm{mg}, 0.5$ mmol), methyl 2-(triphenylphosphoranylidene)acetate $(334.3 \mathrm{mg}, 1.0 \mathrm{mmol})$, $\begin{array}{lllll}\text { palladium } & \text { chloride } & (1.7 & \mathrm{mg}, & 0.01\end{array}$ (9,9-dimethyl-9H-xanthene-4,5-diyl)bis(diphenylphosphine) (11.5 mg, $0.02 \mathrm{mmol}$ ), Potassium carbonate $(69.1 \mathrm{mg}, 0.5 \mathrm{mmol}), 1$,4-dioxane $(3 \mathrm{~mL}), \mathrm{CO}(1 \mathrm{~atm}), 100{ }^{\circ} \mathrm{C}$, $24 \mathrm{~h}$, compound 11 was synthesized and purified on silica gel with EtOAc:PE (V:V= $\left.1: 2, \mathrm{R}_{\mathrm{f}}=0.10\right)$ to give a pale yellow solid $(159 \mathrm{mg}, 68 \%), \mathrm{mp}=175-178{ }^{\circ} \mathrm{C} ;{ }^{1} \mathrm{H}$ NMR $\left(400 \mathrm{MHz}, \mathrm{CDCl}_{3}\right), \delta(\mathrm{ppm})=7.78-7.71(\mathrm{~m}, 8 \mathrm{H}), 7.55-7.44(\mathrm{~m}, 9 \mathrm{H}), 6.86(\mathrm{~d}$, $J=8.8 \mathrm{~Hz}, 2 \mathrm{H}), 3.83(\mathrm{~s}, 3 \mathrm{H}), 3.17(\mathrm{~s}, 3 \mathrm{H}) ;{ }^{13} \mathrm{C} \mathrm{NMR}\left(100 \mathrm{MHz}, \mathrm{CDCl}_{3}\right), \delta(\mathrm{ppm})=$ $192.3\left(\mathrm{~d}, J_{\mathrm{P}-\mathrm{C}}=5.2 \mathrm{~Hz}\right), 168.0\left(\mathrm{~d}, J_{\mathrm{P}-\mathrm{C}}=14.1 \mathrm{~Hz}\right), 161.1,135.2\left(\mathrm{~d}, J_{\mathrm{P}-\mathrm{C}}=9.0 \mathrm{~Hz}\right)$, $133.3\left(\mathrm{~d}, J_{\mathrm{P}-\mathrm{C}}=9.8 \mathrm{~Hz}\right), 131.8\left(\mathrm{~d}, J_{\mathrm{P}-\mathrm{C}}=2.8 \mathrm{~Hz}\right), 130.6,128.6\left(\mathrm{~d}, J_{\mathrm{P}-\mathrm{C}}=12.4 \mathrm{~Hz}\right)$, $126.4\left(\mathrm{~d}, J_{\mathrm{P}-\mathrm{C}}=92.6 \mathrm{~Hz}\right), 112.5,68.4\left(\mathrm{~d}, J_{\mathrm{P}-\mathrm{C}}=111.0 \mathrm{~Hz}\right), 55.3,49.7 ;{ }^{31} \mathrm{P} \mathrm{NMR}(162$ $\left.\mathrm{MHz}, \mathrm{CDCl}_{3}\right), \delta(\mathrm{ppm})=19.3$; HRMS (ESI) Calcd for $\mathrm{C}_{29} \mathrm{H}_{25} \mathrm{O}_{4} \mathrm{P}[\mathrm{M}+\mathrm{H}]^{+}=$ 469.1563, Found $=469.1558$.

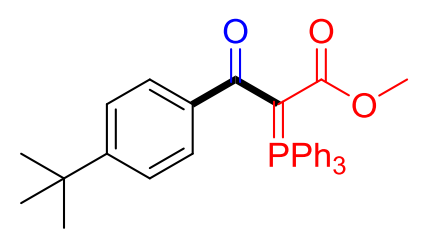

\section{Methyl-3-(4-(tert-butyl)phenyl)-3-oxo-2-(triphenylphosphoranylidene)propanoat}

-e 12. According to the general procedure A, 1-(tert-butyl)-4-iodobenzene (130.0 mg, $0.5 \mathrm{mmol})$, methyl 2-(triphenylphosphoranylidene)acetate $(334.3 \mathrm{mg}, 1.0 \mathrm{mmol}$ ), 
palladium

chloride

$(1.7$

$\mathrm{mg}$,

0.01

mmol),

(9,9-dimethyl-9H-xanthene-4,5-diyl)bis(diphenylphosphine) (11.5 mg, $0.02 \mathrm{mmol}$ ), Potassium carbonate $(69.1 \mathrm{mg}, 0.5 \mathrm{mmol}), 1,4$-dioxane $(3 \mathrm{~mL}), \mathrm{CO}(1 \mathrm{~atm}), 100{ }^{\circ} \mathrm{C}$, $24 \mathrm{~h}$, compound 12 was synthesized and purified on silica gel with EtOAc:PE (V:V = $\left.1: 2, \mathrm{R}_{\mathrm{f}}=0.37\right)$ to give a pale yellow solid $(208 \mathrm{mg}, 84 \%), \mathrm{mp}=208-210{ }^{\circ} \mathrm{C} ;{ }^{1} \mathrm{H}$ NMR (400 MHz, $\left.\mathrm{CDCl}_{3}\right), \delta(\mathrm{ppm})=7.80-7.74(\mathrm{~m}, 6 \mathrm{H}), 7.68(\mathrm{~d}, J=8.3 \mathrm{~Hz}, 2 \mathrm{H})$, 7.55-7.52 (m, $3 \mathrm{H}), 7.49-7.45(\mathrm{~m}, 6 \mathrm{H}), 7.38(\mathrm{~d}, J=8.3 \mathrm{~Hz}, 2 \mathrm{H}), 3.16(\mathrm{~s}, 3 \mathrm{H}), 1.33$ (s, $9 \mathrm{H}) ;{ }^{13} \mathrm{C}$ NMR $\left(100 \mathrm{MHz}, \mathrm{CDCl}_{3}\right), \delta(\mathrm{ppm})=193.0\left(\mathrm{~d}, J_{\mathrm{P}-\mathrm{C}}=5.1 \mathrm{~Hz}\right), 168.0\left(\mathrm{~d}, J_{\mathrm{P}-\mathrm{C}}\right.$ $=14.1 \mathrm{~Hz}), 152.7,139.7\left(\mathrm{~d}, J_{\text {P-C }}=8.7 \mathrm{~Hz}\right), 133.3\left(\mathrm{~d}, J_{\text {P-C }}=9.6 \mathrm{~Hz}\right), 131.8\left(\mathrm{~d}, J_{\text {P-C }}=\right.$ $2.8 \mathrm{~Hz}), 128.6\left(\mathrm{~d}, J_{\mathrm{P}-\mathrm{C}}=12.4 \mathrm{~Hz}\right), 128.4,126.2\left(\mathrm{~d}, J_{\mathrm{P}-\mathrm{C}}=92.8 \mathrm{~Hz}\right), 124.2,68.9(\mathrm{~d}, J$ $\mathrm{P}-\mathrm{C}=110.8 \mathrm{~Hz}), 49.7,34.7,31.3 ;{ }^{31} \mathrm{P} \mathrm{NMR}\left(162 \mathrm{MHz}, \mathrm{CDCl}_{3}\right), \delta(\mathrm{ppm})=19.0$; HRMS (ESI) Calcd for $\mathrm{C}_{32} \mathrm{H}_{31} \mathrm{O}_{3} \mathrm{P}[\mathrm{M}+\mathrm{H}]^{+}=495.2084$, Found $=495.2075$.

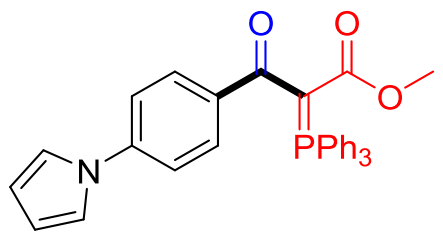

\section{Methyl-3-(4-(1H-pyrrol-1-yl)phenyl)-3-oxo-2-(triphenylphosphoranylidene)prop}

anoate 13. According to the general procedure A, 1-(4-iodophenyl)-1H-pyrrole (134.5 mg, $0.5 \mathrm{mmol}$ ), methyl 2-(triphenylphosphoranylidene)acetate $(267.4 \mathrm{mg}, 0.8 \mathrm{mmol}$ ),

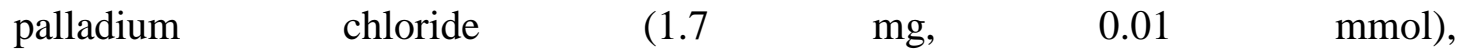
(9,9-dimethyl-9H-xanthene-4,5-diyl)bis(diphenylphosphine) (11.5 mg, $0.02 \mathrm{mmol}$ ), Potassium carbonate $(69.1 \mathrm{mg}, 0.5 \mathrm{mmol}), 1$,4-dioxane $(3 \mathrm{~mL}), \mathrm{CO}(1 \mathrm{~atm}), 100{ }^{\circ} \mathrm{C}$, $24 \mathrm{~h}$, compound 13 was synthesized and purified on silica gel with EtOAc:PE (V:V = $\left.1: 2, \mathrm{R}_{\mathrm{f}}=0.15\right)$ to give a yellow solid $(212 \mathrm{mg}, 84 \%), \mathrm{mp}=162-164{ }^{\circ} \mathrm{C} ;{ }^{1} \mathrm{H} \mathrm{NMR}$ $\left(400 \mathrm{MHz}, \mathrm{CDCl}_{3}\right), \delta(\mathrm{ppm})=7.80-7.75(\mathrm{~m}, 8 \mathrm{H}), 7.56-7.49(\mathrm{~m}, 9 \mathrm{H}), 7.37(\mathrm{~d}, J=8.3$ $\mathrm{Hz}, 2 \mathrm{H}), 7.13$ (s, $2 \mathrm{H}), 6.35$ (s, $2 \mathrm{H}), 3.17$ (s, $3 \mathrm{H}) ;{ }^{13} \mathrm{C} \mathrm{NMR}\left(150 \mathrm{MHz}, \mathrm{CDCl}_{3}\right), \delta$ $(\mathrm{ppm})=192.0\left(\mathrm{~d}, J_{\mathrm{P}-\mathrm{C}}=5.5 \mathrm{~Hz}\right), 168.0\left(\mathrm{~d}, J_{\mathrm{P}-\mathrm{C}}=14.1 \mathrm{~Hz}\right), 141.6,140.1\left(\mathrm{~d}, J_{\mathrm{P}-\mathrm{C}}=\right.$ $8.8 \mathrm{~Hz}), 133.4\left(\mathrm{~d}, J_{\mathrm{P}-\mathrm{C}}=9.8 \mathrm{~Hz}\right), 132.0\left(\mathrm{~d}, J_{\mathrm{P}-\mathrm{C}}=1.8 \mathrm{~Hz}\right), 130.2,128.7\left(\mathrm{~d}, J_{\mathrm{P}-\mathrm{C}}=\right.$ $12.6 \mathrm{~Hz}), 126.1\left(\mathrm{~d}, J_{\mathrm{P}-\mathrm{C}}=92.7 \mathrm{~Hz}\right), 119.3,119.0,110.6,69.3\left(\mathrm{~d}, J_{\mathrm{P}-\mathrm{C}}=110.4 \mathrm{~Hz}\right)$, 49.8; ${ }^{31} \mathrm{P}$ NMR $\left(162 \mathrm{MHz}, \mathrm{CDCl}_{3}\right), \delta(\mathrm{ppm})=19.2$; HRMS (ESI) Calcd for 
$\mathrm{C}_{32} \mathrm{H}_{26} \mathrm{NO}_{3} \mathrm{P}[\mathrm{M}+\mathrm{H}]^{+}=504.1723$, Found $=504.1713$.<smiles>COC(=O)C(=P)C(=P)C(=O)c1ccccc1</smiles>

Methyl 3-oxo-3-phenyl-2-(triphenylphosphoranylidene)propanoate 14. According to the general procedure A, iodobenzene (102.0 $\mathrm{mg}, 0.5 \mathrm{mmol})$, methyl 2-(triphenylphosphoranylidene)acetate (267.4 mg, $0.8 \mathrm{mmol})$, palladium chloride (1.7 mg, $0.01 \mathrm{mmol}),(9,9-$ dimethyl-9H-xanthene-4,5-diyl)bis(diphenylphosphine) (11.5 $\mathrm{mg}, 0.02 \mathrm{mmol})$, Potassium carbonate $(69.1 \mathrm{mg}, 0.5 \mathrm{mmol})$, 1,4-dioxane (3 mL), CO (1 atm), $100{ }^{\circ} \mathrm{C}, 24 \mathrm{~h}$, compound 14 was synthesized and purified on silica gel with EtOAc:PE $\left(\mathrm{V}: \mathrm{V}=1: 2, \mathrm{R}_{\mathrm{f}}=0.16\right)$ to give a pale yellow solid $(213 \mathrm{mg}, 97 \%), \mathrm{mp}=$ 134-137 ${ }^{\circ} \mathrm{C} ;{ }^{1} \mathrm{H}$ NMR $\left(400 \mathrm{MHz}, \mathrm{CDCl}_{3}\right), \delta(\mathrm{ppm})=7.81-7.76(\mathrm{~m}, 6 \mathrm{H}), 7.74-7.72(\mathrm{~m}$, $2 \mathrm{H}), 7.56-7.53(\mathrm{~m}, 3 \mathrm{H}), 7.50-7.46(\mathrm{~m}, 6 \mathrm{H}), 7.38-7.35(\mathrm{~m}, 3 \mathrm{H}), 3.16(\mathrm{~s}, 3 \mathrm{H}) ;{ }^{13} \mathrm{C}$ $\operatorname{NMR}\left(100 \mathrm{MHz}, \mathrm{CDCl}_{3}\right), \delta(\mathrm{ppm})=193.3(\mathrm{~d}, J$ P-C $=5.3 \mathrm{~Hz}), 168.1\left(\mathrm{~d}, J_{\text {P-C }}=14.5\right.$ $\mathrm{Hz}), 143.1\left(\mathrm{~d}, J_{\text {P-C }}=8.8 \mathrm{~Hz}\right), 133.4\left(\mathrm{~d}, J_{\text {P-C }}=9.9 \mathrm{~Hz}\right), 131.9\left(\mathrm{~d}, J_{\text {P-C }}=2.8 \mathrm{~Hz}\right)$, $129.6,128.7\left(\mathrm{~d}, J_{\mathrm{P}-\mathrm{C}}=12.5 \mathrm{~Hz}\right), 128.3,127.3,126.1\left(\mathrm{~d}, J_{\mathrm{P}-\mathrm{C}}=92.7 \mathrm{~Hz}\right), 69.2\left(\mathrm{~d}, J_{\mathrm{P}-\mathrm{C}}\right.$ $=110.9 \mathrm{~Hz}), 49.8 ;{ }^{31} \mathrm{P}$ NMR $\left(162 \mathrm{MHz}, \mathrm{CDCl}_{3}\right), \delta(\mathrm{ppm})=18.9$; HRMS (ESI) Calcd for $\mathrm{C}_{28} \mathrm{H}_{23} \mathrm{O}_{3} \mathrm{P}[\mathrm{M}+\mathrm{H}]^{+}=439.1458$, Found $=439.1451$.<smiles>COC(=O)C(=P)C(=O)c1cccc(C(=O)OC)c1</smiles>

Methyl 3-(3-methoxy-3-oxo-2-(triphenylphosphoranylidene)propanoyl)benzoate

15. According to the general procedure A, methyl 3 -iodobenzoate $(131.0 \mathrm{mg}, 0.5$ mmol), methyl 2-(triphenylphosphoranylidene)acetate (200.6 mg, $0.6 \mathrm{mmol}$ ), palladium chloride $\quad\left(\begin{array}{llll}1.7 & \mathrm{mg}, & 0.01 & \mathrm{mmol}) \text {, }\end{array}\right.$ (9,9-dimethyl-9H-xanthene-4,5-diyl)bis(diphenylphosphine) (11.5 mg, $0.02 \mathrm{mmol}$ ), Potassium carbonate (69.1 mg, $0.5 \mathrm{mmol}), 1$,4-dioxane $(3 \mathrm{~mL}), \mathrm{CO}(1 \mathrm{~atm}), 100{ }^{\circ} \mathrm{C}$, $12 \mathrm{~h}$, compound 15 was synthesized and purified on silica gel with EtOAc:PE (V:V = $\left.1: 2, \mathrm{R}_{\mathrm{f}}=0.11\right)$ to give a yellow solid $(233 \mathrm{mg}, 94 \%), \mathrm{mp}=54-56{ }^{\circ} \mathrm{C} ;{ }^{1} \mathrm{H}$ NMR $(400$ $\left.\mathrm{MHz}, \mathrm{CDCl}_{3}\right), \delta(\mathrm{ppm})=8.36(\mathrm{t}, J=1.4 \mathrm{~Hz}, 1 \mathrm{H}), 8.05(\mathrm{dt}, J=7.7 \mathrm{~Hz}, J=1.2 \mathrm{~Hz}, 1$ 
H), $7.86(\mathrm{~d}, J=7.6 \mathrm{~Hz}, 1 \mathrm{H})$, 7.79-7.74 (m, $6 \mathrm{H}), 7.57-7.53(\mathrm{~m}, 3 \mathrm{H}), 7.50-7.46(\mathrm{~m}, 6$ H), $7.41(\mathrm{t}, J=7.7 \mathrm{~Hz}, 1 \mathrm{H}), 3.89$ (s, $3 \mathrm{H}), 3.13$ (s, $3 \mathrm{H}) ;{ }^{13} \mathrm{C} \mathrm{NMR}\left(100 \mathrm{MHz}, \mathrm{CDCl}_{3}\right)$, $\delta(\mathrm{ppm})=192.0\left(\mathrm{~d}, J_{\mathrm{P}-\mathrm{C}}=5.3 \mathrm{~Hz}\right), 167.8\left(\mathrm{~d}, J_{\mathrm{P}-\mathrm{C}}=14.1 \mathrm{~Hz}\right), 167.1,143.3\left(\mathrm{~d}, J_{\mathrm{P}-\mathrm{C}}=\right.$ $9.0 \mathrm{~Hz}), 133.3\left(\mathrm{~d}, J_{\mathrm{P}-\mathrm{C}}=9.7 \mathrm{~Hz}\right), 132.7,132.0\left(\mathrm{~d}, J_{\mathrm{P}-\mathrm{C}}=2.9 \mathrm{~Hz}\right), 130.4,129.5,129.2$, $128.6\left(\mathrm{~d}, J_{\text {P-C }}=12.5 \mathrm{~Hz}\right), 127.4,125.8\left(\mathrm{~d}, J_{\text {P-C }}=92.7 \mathrm{~Hz}\right), 69.8\left(\mathrm{~d}, J_{\text {P-C }}=110.5 \mathrm{~Hz}\right)$, 52.0, 49.8; ${ }^{31} \mathrm{P}$ NMR (162 MHz, $\left.\mathrm{CDCl}_{3}\right), \delta(\mathrm{ppm})=18.9$; HRMS (ESI) Calcd for $\mathrm{C}_{30} \mathrm{H}_{25} \mathrm{O}_{5} \mathrm{P}[\mathrm{M}+\mathrm{H}]^{+}=497.1512$, Found $=497.1514$.

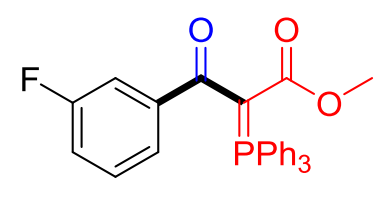

Methyl 3-(3-fluorophenyl)-3-oxo-2-(triphenylphosphoranylidene)propanoate 16.

According to the general procedure A, 1-fluoro-3-iodobenzene $(111.0 \mathrm{mg}, 0.5 \mathrm{mmol})$, methyl 2-(triphenylphosphoranylidene)acetate (200.6 mg, $0.6 \mathrm{mmol})$, palladium chloride (1.7 $\mathrm{mg}, 0.01 \quad \mathrm{mmol})$, (9,9-dimethyl-9H-xanthene-4,5-diyl)bis(diphenylphosphine) (11.5 mg, $0.02 \mathrm{mmol}$ ), Potassium carbonate $(69.1 \mathrm{mg}, 0.5 \mathrm{mmol}), 1$,4-dioxane $(3 \mathrm{~mL}), \mathrm{CO}(1 \mathrm{~atm}), 100{ }^{\circ} \mathrm{C}$, $12 \mathrm{~h}$, compound 16 was synthesized and purified on silica gel with EtOAc:PE $(\mathrm{V}: \mathrm{V}=$ $\left.1: 2, \mathrm{R}_{\mathrm{f}}=0.27\right)$ to give a colorless solid $(221 \mathrm{mg}, 97 \%), \mathrm{mp}=150-152{ }^{\circ} \mathrm{C} ;{ }^{1} \mathrm{H} \mathrm{NMR}$ $\left(400 \mathrm{MHz}, \mathrm{CDCl}_{3}\right), \delta(\mathrm{ppm})=7.77-7.72(\mathrm{~m}, 6 \mathrm{H}), 7.58-7.44(\mathrm{~m}, 10 \mathrm{H})$, 7.36-7.29 (m, $2 \mathrm{H}), 7.05(\mathrm{td}, J=8.3 \mathrm{~Hz}, J=1.9 \mathrm{~Hz}, 1 \mathrm{H}), 3.15(\mathrm{~s}, 3 \mathrm{H}) ;{ }^{13} \mathrm{C}$ NMR $(100 \mathrm{MHz}$, $\left.\mathrm{CDCl}_{3}\right), \delta(\mathrm{ppm})=191.7\left(\mathrm{~d}, J_{\text {P-C }}=5.2 \mathrm{~Hz}\right), 167.8\left(\mathrm{~d}, J_{\text {P-C }}=14.5 \mathrm{~Hz}\right), 162.1\left(\mathrm{~d}, J_{\text {F-C }}\right.$ $=243.5 \mathrm{~Hz}), 145.4\left(\mathrm{q}, J_{\mathrm{F}-\mathrm{C}}=6.3 \mathrm{~Hz}\right), 133.3\left(\mathrm{~d}, J_{\mathrm{P}-\mathrm{C}}=9.7 \mathrm{~Hz}\right), 132.0\left(\mathrm{~d}, J_{\mathrm{P}-\mathrm{C}}=2.9\right.$ $\mathrm{Hz}), 128.6\left(\mathrm{~d}, J_{\mathrm{P}-\mathrm{C}}=12.6 \mathrm{~Hz}\right), 128.6,125.8\left(\mathrm{~d}, J_{\mathrm{P}-\mathrm{C}}=92.7 \mathrm{~Hz}\right), 123.8\left(\mathrm{~d}, J_{\mathrm{F}-\mathrm{C}}=2.6\right.$ $\mathrm{Hz}), 116.1\left(\mathrm{~d}, J_{\mathrm{F}-\mathrm{C}}=21.3 \mathrm{~Hz}\right), 115.0\left(\mathrm{~d}, J_{\mathrm{F}-\mathrm{C}}=22.1 \mathrm{~Hz}\right), 69.5\left(\mathrm{~d}, J_{\mathrm{P}-\mathrm{C}}=111.1 \mathrm{~Hz}\right)$, 49.8; ${ }^{31} \mathrm{P}$ NMR $\left(162 \mathrm{MHz}, \mathrm{CDCl}_{3}\right), \delta(\mathrm{ppm})=18.9$; HRMS (ESI) Calcd for $\mathrm{C}_{28} \mathrm{H}_{22} \mathrm{FO}_{3} \mathrm{P}[\mathrm{M}+\mathrm{H}]^{+}=457.1363$, Found $=457.1363$.<smiles>COC(=O)C(=Pc1ccccc1)C(=O)c1cccc(Cl)c1</smiles>

Methyl 3-(3-chlorophenyl)-3-oxo-2-(triphenylphosphoranylidene)propanoate 17. 
According to the general procedure A, 1-chloro-3-iodobenzene (119.2 mg, $0.5 \mathrm{mmol})$, methyl 2-(triphenylphosphoranylidene)acetate (200.6 mg, $0.6 \mathrm{mmol})$, palladium chloride

$\mathrm{mg}$,

0.01

mmol),

(9,9-dimethyl-9H-xanthene-4,5-diyl)bis(diphenylphosphine) (11.5 mg, $0.02 \mathrm{mmol}$ ), Potassium carbonate (69.1 mg, $0.5 \mathrm{mmol}), 1$,4-dioxane $(3 \mathrm{~mL}), \mathrm{CO}(1 \mathrm{~atm}), 100{ }^{\circ} \mathrm{C}$, $12 \mathrm{~h}$, compound 17 was synthesized and purified on silica gel with EtOAc:PE (V:V = $\left.1: 2, \mathrm{R}_{\mathrm{f}}=0.33\right)$ to give a colorless solid $(163 \mathrm{mg}, 69 \%), \mathrm{mp}=136-139{ }^{\circ} \mathrm{C} ;{ }^{1} \mathrm{H} \mathrm{NMR}$ $\left(400 \mathrm{MHz}, \mathrm{CDCl}_{3}\right), \delta(\mathrm{ppm})=7.77-7.72(\mathrm{~m}, 6 \mathrm{H}), 7.63(\mathrm{t}, J=1.6 \mathrm{~Hz}, 1 \mathrm{H}), 7.58-7.54$ (m, $4 \mathrm{H})$, 7.51-7.47 (m, $6 \mathrm{H})$, 7.34-7.31 (m, $1 \mathrm{H})$, 7.29-7.25 (m, $1 \mathrm{H}), 3.15(\mathrm{~s}, 3 \mathrm{H})$; ${ }^{13} \mathrm{C}$ NMR $\left(100 \mathrm{MHz}, \mathrm{CDCl}_{3}\right), \delta(\mathrm{ppm})=191.5\left(\mathrm{~d}, J_{\mathrm{P}-\mathrm{C}}=5.3 \mathrm{~Hz}\right), 167.8\left(\mathrm{~d}, J_{\mathrm{P}-\mathrm{C}}=\right.$ $14.5 \mathrm{~Hz}), 144.8\left(\mathrm{~d}, J_{\mathrm{P}-\mathrm{C}}=9.0 \mathrm{~Hz}\right), 133.3\left(\mathrm{~d}, J_{\mathrm{P}-\mathrm{C}}=9.9 \mathrm{~Hz}\right), 133.2,132.0\left(\mathrm{~d}, J_{\text {P-C }}=\right.$ $2.9 \mathrm{~Hz}), 129.3,128.7\left(\mathrm{~d}, J_{\mathrm{P}-\mathrm{C}}=12.5 \mathrm{~Hz}\right), 128.5,128.3,126.3,125.7\left(\mathrm{~d}, J_{\mathrm{P}-\mathrm{C}}=92.8\right.$ $\mathrm{Hz}), 69.6\left(\mathrm{~d}, J_{\mathrm{P}-\mathrm{C}}=110.9 \mathrm{~Hz}\right), 49.8 ;{ }^{31} \mathrm{P} \mathrm{NMR}\left(162 \mathrm{MHz}, \mathrm{CDCl}_{3}\right), \delta(\mathrm{ppm})=18.9$; HRMS (ESI) Calcd for $\mathrm{C}_{28} \mathrm{H}_{22} \mathrm{ClO}_{3} \mathrm{P}[\mathrm{M}+\mathrm{H}]^{+}=473.1068$, Found $=473.1066$.<smiles>COC(=O)C(=P)C(=O)c1cccc(Br)c1</smiles>

Methyl 3-(3-bromophenyl)-3-oxo-2-(triphenylphosphoranylidene)propanoate 18. According to the general procedure A, 1-bromo-3-iodobenzene (141.4 mg, $0.5 \mathrm{mmol}$ ), methyl 2-(triphenylphosphoranylidene)acetate (200.6 mg, $0.6 \mathrm{mmol})$, palladium chloride $\mathrm{mg}$, 0.01 mmol), (9,9-dimethyl-9H-xanthene-4,5-diyl)bis(diphenylphosphine) (11.5 mg, $0.02 \mathrm{mmol}$ ), Potassium carbonate $(69.1 \mathrm{mg}, 0.5 \mathrm{mmol}), 1$,4-dioxane $(3 \mathrm{~mL}), \mathrm{CO}(1 \mathrm{~atm}), 100{ }^{\circ} \mathrm{C}$, $12 \mathrm{~h}$, compound 18 was synthesized and purified on silica gel with EtOAc:PE (V:V = $\left.1: 2, \mathrm{R}_{\mathrm{f}}=0.33\right)$ to give a colorless solid $(181 \mathrm{mg}, 70 \%), \mathrm{mp}=119-122{ }^{\circ} \mathrm{C} ;{ }^{1} \mathrm{H}$ NMR $\left(400 \mathrm{MHz}, \mathrm{CDCl}_{3}\right), \delta(\mathrm{ppm})=7.80-7.73(\mathrm{~m}, 7 \mathrm{H}), 7.61(\mathrm{~d}, J=7.6 \mathrm{~Hz}, 1 \mathrm{H}), 7.58-7.54$ $(\mathrm{m}, 3 \mathrm{H}), 7.51-7.47(\mathrm{~m}, 7 \mathrm{H}), 7.22(\mathrm{t}, J=7.8 \mathrm{~Hz}, 1 \mathrm{H}), 3.17(\mathrm{~s}, 3 \mathrm{H}) ;{ }^{13} \mathrm{C}$ NMR $(100$ $\left.\mathrm{MHz}, \mathrm{CDCl}_{3}\right), \delta(\mathrm{ppm})=191.3\left(\mathrm{~d}, J_{\mathrm{P}-\mathrm{C}}=5.5 \mathrm{~Hz}\right), 167.8\left(\mathrm{~d}, J_{\mathrm{P}-\mathrm{C}}=14.4 \mathrm{~Hz}\right), 145.0(\mathrm{~d}$, $\left.J_{\text {P-C }}=9.2 \mathrm{~Hz}\right), 133.3\left(\mathrm{~d}, J_{\text {P-C }}=9.7 \mathrm{~Hz}\right), 132.2,132.0\left(\mathrm{~d}, J_{\text {P-C }}=2.9 \mathrm{~Hz}\right), 131.1,128.8$, $128.7\left(\mathrm{~d}, J_{\mathrm{P}-\mathrm{C}}=12.6 \mathrm{~Hz}\right), 126.8,125.6\left(\mathrm{~d}, J_{\mathrm{P}-\mathrm{C}}=92.9 \mathrm{~Hz}\right), 121.4,69.6\left(\mathrm{~d}, J_{\mathrm{P}-\mathrm{C}}=\right.$ 
110.7 Hz), 49.9; ${ }^{31} \mathrm{P}$ NMR (162 MHz, $\left.\mathrm{CDCl}_{3}\right), \delta(\mathrm{ppm})=18.9$; HRMS (ESI) Calcd for $\mathrm{C}_{28} \mathrm{H}_{22} \mathrm{BrO}_{3} \mathrm{P}[\mathrm{M}+\mathrm{H}]^{+}=517.0563$, Found $=517.0560$.<smiles>COC(=O)C(=Pc1ccccc1)C(=O)c1cccc([N+](=O)[O-])c1</smiles>

Methyl 3-(3-nitrophenyl)-3-oxo-2-(triphenylphosphoranylidene)propanoate 19.

According to the general procedure A, 1-iodo-3-nitrobenzene $(124.5 \mathrm{mg}, 0.5 \mathrm{mmol})$, methyl 2-(triphenylphosphoranylidene)acetate (200.6 mg, $0.6 \mathrm{mmol})$, palladium chloride $\quad(1.7 \quad \mathrm{mg}, 0.01 \quad \mathrm{mmol})$, (9,9-dimethyl-9H-xanthene-4,5-diyl)bis(diphenylphosphine) (11.5 mg, $0.02 \mathrm{mmol}$ ), Potassium carbonate $(69.1 \mathrm{mg}, 0.5 \mathrm{mmol}), 1$,4-dioxane $(3 \mathrm{~mL}), \mathrm{CO}(1 \mathrm{~atm}), 100{ }^{\circ} \mathrm{C}$, $12 \mathrm{~h}$, compound 19 was synthesized and purified on silica gel with EtOAc:PE (V:V = $\left.1: 2, \mathrm{R}_{\mathrm{f}}=0.17\right)$ to give a pale yellow solid $(225 \mathrm{mg}, 93 \%), \mathrm{mp}=143-145{ }^{\circ} \mathrm{C} ;{ }^{1} \mathrm{H}$ NMR $\left(400 \mathrm{MHz}, \mathrm{CDCl}_{3}\right), \delta(\mathrm{ppm})=8.52(\mathrm{t}, J=1.8 \mathrm{~Hz}, 1 \mathrm{H}), 8.22-8.19(\mathrm{~m}, 1 \mathrm{H})$, $7.97(\mathrm{~d}, J=7.6 \mathrm{~Hz}, 1 \mathrm{H}), 7.80-7.75(\mathrm{~m}, 6 \mathrm{H}), 7.60-7.48(\mathrm{~m}, 10 \mathrm{H}), 3.12(\mathrm{~s}, 3 \mathrm{H}) ;{ }^{13} \mathrm{C}$ NMR $\left(100 \mathrm{MHz}, \mathrm{CDCl}_{3}\right), \delta(\mathrm{ppm})=190.4\left(\mathrm{~d}, J_{\mathrm{P}-\mathrm{C}}=5.8 \mathrm{~Hz}\right), 167.6\left(\mathrm{~d}, J_{\text {P-C }}=13.5\right.$ $\mathrm{Hz}), 147.4,144.6\left(\mathrm{~d}, J_{\text {P-C }}=9.5 \mathrm{~Hz}\right), 134.2,133.3\left(\mathrm{~d}, J_{\text {P-C }}=9.7 \mathrm{~Hz}\right), 132.2\left(\mathrm{~d}, J_{\text {P-C }}=\right.$ $2.9 \mathrm{~Hz}), 128.8\left(\mathrm{~d}, J_{\text {P-C }}=12.6 \mathrm{~Hz}\right), 128.2,125.8,124.4\left(\mathrm{~d}, J_{\text {P-C }}=94.7 \mathrm{~Hz}\right), 123.4$, $70.3\left(\mathrm{~d}, J_{\mathrm{P}-\mathrm{C}}=109.7 \mathrm{~Hz}\right), 49.8 ;{ }^{31} \mathrm{P}$ NMR $\left(162 \mathrm{MHz}, \mathrm{CDCl}_{3}\right), \delta(\mathrm{ppm})=19.0 ; \mathrm{HRMS}$ (ESI) Calcd for $\mathrm{C}_{28} \mathrm{H}_{22} \mathrm{NO}_{5} \mathrm{P}[\mathrm{M}+\mathrm{H}]^{+}=484.1308$, Found $=484.1304$.<smiles>COC(=O)C(=Pc1ccccc1)C(=O)c1cccc(C)c1</smiles>

Methyl 3-oxo-3-(m-tolyl)-2-(triphenylphosphoranylidene)propanoate 20. According to the general procedure A, 1-iodo-3-methylbenzene (109.0 mg, $0.5 \mathrm{mmol})$, methyl 2-(triphenylphosphoranylidene)acetate (267.4 mg, $0.8 \mathrm{mmol})$, palladium chloride (1.7 $\mathrm{mg}$, 0.01 mmol), (9,9-dimethyl-9H-xanthene-4,5-diyl)bis(diphenylphosphine) (11.5 mg, $0.02 \mathrm{mmol}$ ), Potassium carbonate $(69.1 \mathrm{mg}, 0.5 \mathrm{mmol}), 1,4$-dioxane $(3 \mathrm{~mL}), \mathrm{CO}(1 \mathrm{~atm}), 100{ }^{\circ} \mathrm{C}$, $12 \mathrm{~h}$, compound 20 was synthesized and purified on silica gel with EtOAc:PE $(\mathrm{V}: \mathrm{V}=$ 
$\left.1: 2, \mathrm{R}_{\mathrm{f}}=0.18\right)$ to give a pale yellow solid $(165 \mathrm{mg}, 73 \%), \mathrm{mp}=142-145{ }^{\circ} \mathrm{C} ;{ }^{1} \mathrm{H}$ NMR $\left(400 \mathrm{MHz}, \mathrm{CDCl}_{3}\right), \delta(\mathrm{ppm})=7.80-7.74(\mathrm{~m}, 6 \mathrm{H}), 7.56-7.52(\mathrm{~m}, 4 \mathrm{H})$, 7.50-7.45 (m, $7 \mathrm{H}), 7.24-7.17(\mathrm{~m}, 2 \mathrm{H}), 3.16$ (s, $3 \mathrm{H}), 2.36(\mathrm{~s}, 3 \mathrm{H}) ;{ }^{13} \mathrm{C}$ NMR $(100$ $\left.\mathrm{MHz} \mathrm{CDCl}_{3}\right), \delta(\mathrm{ppm})=193.4\left(\mathrm{~d}, J_{\mathrm{P}-\mathrm{C}}=5.1 \mathrm{~Hz}\right), 168.0\left(\mathrm{~d}, J_{\mathrm{P}-\mathrm{C}}=14.7 \mathrm{~Hz}\right), 142.9(\mathrm{~d}$, $\left.J_{\mathrm{P}-\mathrm{C}}=8.8 \mathrm{~Hz}\right), 136.8,133.3\left(\mathrm{~d}, J_{\mathrm{P}-\mathrm{C}}=9.7 \mathrm{~Hz}\right), 131.8\left(\mathrm{~d}, J_{\mathrm{P}-\mathrm{C}}=2.9 \mathrm{~Hz}\right), 130.3,128.6$ $\left(\mathrm{d}, J_{\text {P-C }}=12.4 \mathrm{~Hz}\right), 128.6,127.0,126.1\left(\mathrm{~d}, J_{\text {P-C }}=92.7 \mathrm{~Hz}\right), 125.5,69.2\left(\mathrm{~d}, J_{\text {P-C }}=\right.$ 111.0 Hz), 49.7, 21.4; ${ }^{31} \mathrm{P}$ NMR (162 $\left.\mathrm{MHz}, \mathrm{CDCl}_{3}\right), \delta(\mathrm{ppm})=18.9$; HRMS (ESI) Calcd for $\mathrm{C}_{29} \mathrm{H}_{25} \mathrm{O}_{3} \mathrm{P}[\mathrm{M}+\mathrm{H}]^{+}=453.1614$, Found $=453.1609$.

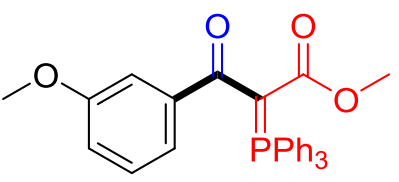

\section{Methyl 3-(3-methoxyphenyl)-3-oxo-2-(triphenylphosphoranylidene)propanoate}

21. According to the general procedure A, 1-iodo-3-methoxybenzene (117.0 mg, 0.5 mmol), methyl 2-(triphenylphosphoranylidene)acetate $(267.4 \mathrm{mg}, 0.8 \mathrm{mmol}$ ), palladium chloride $\quad(1.7 \quad \mathrm{mg}, \quad 0.01 \quad \mathrm{mmol})$, (9,9-dimethyl-9H-xanthene-4,5-diyl)bis(diphenylphosphine) (11.5 mg, $0.02 \mathrm{mmol}$ ), Potassium carbonate (69.1 mg, $0.5 \mathrm{mmol}), 1$,4-dioxane $(3 \mathrm{~mL}), \mathrm{CO}(1 \mathrm{~atm}), 100{ }^{\circ} \mathrm{C}$, $12 \mathrm{~h}$, compound 21 was synthesized and purified on silica gel with EtOAc:PE (V:V = $\left.1: 2, \mathrm{R}_{\mathrm{f}}=0.12\right)$ to give a pale yellow solid $(204 \mathrm{mg}, 87 \%), \mathrm{mp}=149-152{ }^{\circ} \mathrm{C} ;{ }^{1} \mathrm{H}$ NMR $\left(400 \mathrm{MHz}, \mathrm{CDCl}_{3}\right), \delta(\mathrm{ppm})=7.79-7.74(\mathrm{~m}, 6 \mathrm{H}), 7.56-7.52(\mathrm{~m}, 3 \mathrm{H})$, 7.50-7.45 (m, $6 \mathrm{H}), 7.34$ (d, $J=7.5 \mathrm{~Hz}, 1 \mathrm{H}), 7.28-7.22(\mathrm{~m}, 2 \mathrm{H}), 6.93$ (dd, $J=8.0 \mathrm{~Hz}$, $J=1.9 \mathrm{~Hz}, 1 \mathrm{H}), 3.81(\mathrm{~s}, 3 \mathrm{H}), 3.17(\mathrm{~s}, 3 \mathrm{H}) ;{ }^{13} \mathrm{C} \mathrm{NMR}\left(100 \mathrm{MHz}, \mathrm{CDCl}_{3}\right), \delta(\mathrm{ppm})=$ $192.9\left(\mathrm{~d}, J_{\mathrm{P}-\mathrm{C}}=5.4 \mathrm{~Hz}\right), 168.1\left(\mathrm{~d}, J_{\mathrm{P}-\mathrm{C}}=14.6 \mathrm{~Hz}\right), 159.0,144.5\left(\mathrm{~d}, J_{\mathrm{P}-\mathrm{C}}=8.8 \mathrm{~Hz}\right)$, $133.4\left(\mathrm{~d}, J_{\mathrm{P}-\mathrm{C}}=9.7 \mathrm{~Hz}\right), 132.0\left(\mathrm{~d}, J_{\mathrm{P}-\mathrm{C}}=2.8 \mathrm{~Hz}\right), 128.7\left(\mathrm{~d}, J_{\mathrm{P}-\mathrm{C}}=12.4 \mathrm{~Hz}\right), 128.2$, $126.0\left(\mathrm{~d}, J_{\mathrm{P}-\mathrm{C}}=92.7 \mathrm{~Hz}\right), 121.1,115.9,112.8,69.2\left(\mathrm{~d}, J_{\mathrm{P}-\mathrm{C}}=111.1 \mathrm{~Hz}\right), 55.3,49.9$; ${ }^{31} \mathrm{P}$ NMR $\left(162 \mathrm{MHz}, \mathrm{CDCl}_{3}\right), \delta(\mathrm{ppm})=18.9$; HRMS (ESI) Calcd for $\mathrm{C}_{29} \mathrm{H}_{25} \mathrm{O}_{4} \mathrm{P}[\mathrm{M}$ $+\mathrm{H}]^{+}=469.1563$, Found $=469.1553$.

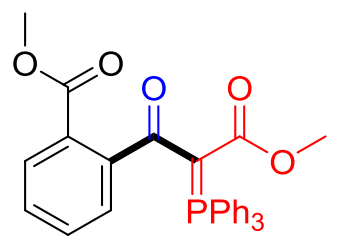




\section{Methyl 2-(3-methoxy-3-oxo-2-(triphenylphosphoranylidene)propanoyl)benzoate}

22. According to the general procedure A, methyl 2-iodobenzoate $(131.0 \mathrm{mg}, 0.5$ mmol), methyl 2-(triphenylphosphoranylidene)acetate (200.6 mg, $0.6 \mathrm{mmol}$ ),

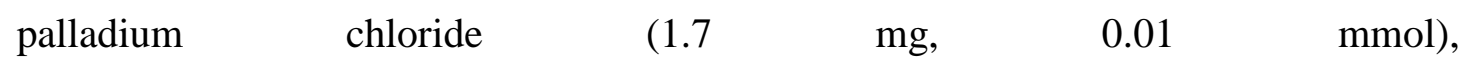
(9,9-dimethyl-9H-xanthene-4,5-diyl)bis(diphenylphosphine) (11.5 mg, $0.02 \mathrm{mmol}$ ), Potassium carbonate $(69.1 \mathrm{mg}, 0.5 \mathrm{mmol}), 1,4$-dioxane $(3 \mathrm{~mL}), \mathrm{CO}(1 \mathrm{~atm}), 100{ }^{\circ} \mathrm{C}$, $12 \mathrm{~h}$, compound 22 was synthesized and purified on silica gel with EtOAc:PE (V:V = $\left.1: 2, \mathrm{R}_{\mathrm{f}}=0.10\right)$ to give a pale yellow solid $(60 \mathrm{mg}, 24 \%), \mathrm{mp}=170-172{ }^{\circ} \mathrm{C} ;{ }^{1} \mathrm{H} \mathrm{NMR}$ $\left(400 \mathrm{MHz}, \mathrm{CDCl}_{3}\right), \delta(\mathrm{ppm})=7.91-7.82(\mathrm{~m}, 7 \mathrm{H}), 7.58-7.45(\mathrm{~m}, 10 \mathrm{H}), 7.31(\mathrm{t}, J=$ $6.9 \mathrm{~Hz}, 2 \mathrm{H}), 3.83(\mathrm{~s}, 3 \mathrm{H}), 3.02(\mathrm{~s}, 3 \mathrm{H}) ;{ }^{13} \mathrm{C} \mathrm{NMR}\left(100 \mathrm{MHz}, \mathrm{CDCl}_{3}\right), \delta(\mathrm{ppm})=$ $192.8\left(\mathrm{~d}, J_{\text {P-C }}=5.8 \mathrm{~Hz}\right), 167.7\left(\mathrm{~d}, J_{\text {P-C }}=15.1 \mathrm{~Hz}\right), 167.3,146.6\left(\mathrm{~d}, J_{\text {P-C }}=9.4 \mathrm{~Hz}\right)$, $133.5\left(\mathrm{~d}, J_{\mathrm{P}-\mathrm{C}}=9.9 \mathrm{~Hz}\right), 131.9\left(\mathrm{~d}, J_{\mathrm{P}-\mathrm{C}}=2.9 \mathrm{~Hz}\right), 131.4,129.6,128.6\left(\mathrm{~d}, J_{\mathrm{P}-\mathrm{C}}=12.7\right.$ $\mathrm{Hz}), 127.9,127.1,126.9,126.0\left(\mathrm{~d}, J_{\mathrm{P}-\mathrm{C}}=92.7 \mathrm{~Hz}\right), 71.4\left(\mathrm{~d}, J_{\mathrm{P}-\mathrm{C}}=111.4 \mathrm{~Hz}\right), 51.9$, 49.7; ${ }^{31} \mathrm{P}$ NMR $\left(162 \mathrm{MHz}, \mathrm{CDCl}_{3}\right), \delta(\mathrm{ppm})=17.4$; HRMS (ESI) Calcd for $\mathrm{C}_{30} \mathrm{H}_{25} \mathrm{O}_{5} \mathrm{P}[\mathrm{M}+\mathrm{H}]^{+}=497.1512$, Found $=497.1514$.<smiles>COC(=O)C(=P)C(=O)c1ccccc1F</smiles>

Methyl 3-(2-fluorophenyl)-3-oxo-2-(triphenylphosphoranylidene)propanoate 23. According to the general procedure A, 1-fluoro-2-iodobenzene (111.0 mg, $0.5 \mathrm{mmol})$, methyl 2-(triphenylphosphoranylidene)acetate $(200.6 \mathrm{mg}, 0.6 \mathrm{mmol})$, palladium chloride

$\mathrm{mg}$, 0.01 mmol), (9,9-dimethyl-9H-xanthene-4,5-diyl)bis(diphenylphosphine) (11.5 mg, $0.02 \mathrm{mmol}$ ), Potassium carbonate $(69.1 \mathrm{mg}, 0.5 \mathrm{mmol}), 1$,4-dioxane $(3 \mathrm{~mL}), \mathrm{CO}(1 \mathrm{~atm}), 100{ }^{\circ} \mathrm{C}$, $12 \mathrm{~h}$, compound 23 was synthesized and purified on silica gel with EtOAc:PE (V:V = $\left.1: 2, \mathrm{R}_{\mathrm{f}}=0.19\right)$ to give a colorless solid $(139 \mathrm{mg}, 61 \%), \mathrm{mp}=165-168{ }^{\circ} \mathrm{C} ;{ }^{1} \mathrm{H} \mathrm{NMR}$ $\left(400 \mathrm{MHz}, \mathrm{CDCl}_{3}\right), \delta(\mathrm{ppm})=7.79-7.74(\mathrm{~m}, 6 \mathrm{H}), 7.58-7.47(\mathrm{~m}, 9 \mathrm{H}), 7.40(\mathrm{td}, J=$ $7.3 \mathrm{~Hz}, J=1.6 \mathrm{~Hz}, 1 \mathrm{H}), 7.31-7.27(\mathrm{~m}, 1 \mathrm{H}), 7.11(\mathrm{t}, J=7.0 \mathrm{~Hz}, 1 \mathrm{H}), 7.02(\mathrm{t}, J=9.5$ $\mathrm{Hz}, 1 \mathrm{H}), 3.11(\mathrm{~s}, 3 \mathrm{H}) ;{ }^{13} \mathrm{C} \mathrm{NMR}\left(100 \mathrm{MHz}, \mathrm{CDCl}_{3}\right), \delta(\mathrm{ppm})=187.6\left(\mathrm{~d}, J_{\text {P-C }}=6.2\right.$ $\mathrm{Hz}), 167.6\left(\mathrm{~d}, J_{\mathrm{P}-\mathrm{C}}=14.7 \mathrm{~Hz}\right), 159.3\left(\mathrm{~d}, J_{\mathrm{F}-\mathrm{C}}=244.5 \mathrm{~Hz}\right), 133.5\left(\mathrm{~d}, J_{\mathrm{P}-\mathrm{C}}=9.9 \mathrm{~Hz}\right)$, 
$132.2\left(\mathrm{~d}, J_{\mathrm{F}-\mathrm{C}}=8.9 \mathrm{~Hz}\right), 132.0\left(\mathrm{~d}, J_{\mathrm{P}-\mathrm{C}}=2.9 \mathrm{~Hz}\right), 130.0\left(\mathrm{~d}, J_{\mathrm{F}-\mathrm{C}}=8.1 \mathrm{~Hz}\right), 129.0(\mathrm{~d}$, $\left.J_{\mathrm{F}-\mathrm{C}}=4.0 \mathrm{~Hz}\right), 128.6\left(\mathrm{~d}, J_{\mathrm{P}-\mathrm{C}}=12.7 \mathrm{~Hz}\right), 125.7\left(\mathrm{~d}, J_{\mathrm{P}-\mathrm{C}}=93.1 \mathrm{~Hz}\right), 123.6\left(\mathrm{~d}, J_{\mathrm{F}-\mathrm{C}}=\right.$ $3.1 \mathrm{~Hz}), 114.7\left(\mathrm{~d}, J_{\mathrm{F}-\mathrm{C}}=22.5 \mathrm{~Hz}\right), 72.5\left(\mathrm{~d}, J_{\mathrm{P}-\mathrm{C}}=112.5 \mathrm{~Hz}\right), 49.8 ;{ }^{31} \mathrm{P}$ NMR $(162$ $\left.\mathrm{MHz}, \mathrm{CDCl}_{3}\right), \delta(\mathrm{ppm})=17.9$; HRMS (ESI) Calcd for $\mathrm{C}_{28} \mathrm{H}_{22} \mathrm{FO}_{3} \mathrm{P}[\mathrm{M}+\mathrm{H}]^{+}=$ 457.1363, Found $=457.1363$.<smiles>COC(=O)C(=P)C(=O)c1ccccc1Cl</smiles>

Methyl 3-(2-chlorophenyl)-3-oxo-2-(triphenylphosphoranylidene)propanoate 24.

According to the general procedure A, 1-chloro-2-iodobenzene (119.2 mg, $0.5 \mathrm{mmol})$, methyl 2-(triphenylphosphoranylidene)acetate $(267.4 \mathrm{mg}, 0.8 \mathrm{mmol})$, palladium chloride

$\mathrm{mg}$,

0.01

mmol),

(9,9-dimethyl-9H-xanthene-4,5-diyl)bis(diphenylphosphine) (11.5 mg, $0.02 \mathrm{mmol}$ ), Potassium carbonate $(69.1 \mathrm{mg}, 0.5 \mathrm{mmol}), 1,4$-dioxane $(3 \mathrm{~mL}), \mathrm{CO}(1 \mathrm{~atm}), 100{ }^{\circ} \mathrm{C}$, $12 \mathrm{~h}$, compound 24 was synthesized and purified on silica gel with EtOAc:PE (V:V = $\left.1: 2, \mathrm{R}_{\mathrm{f}}=0.14\right)$ to give a colorless solid $(182 \mathrm{mg}, 77 \%), \mathrm{mp}=175-179{ }^{\circ} \mathrm{C} ;{ }^{1} \mathrm{H} \mathrm{NMR}$ $\left(400 \mathrm{MHz}, \mathrm{CDCl}_{3}\right), \delta(\mathrm{ppm})=7.83-7.78(\mathrm{~m}, 6 \mathrm{H}), 7.58-7.54(\mathrm{~m}, 3 \mathrm{H})$, 7.52-7.47 (m, 6 H), $7.32(\mathrm{td}, J=7.6 \mathrm{~Hz}, \mathrm{~J}=1.3 \mathrm{~Hz}, 2 \mathrm{H}), 7.25-7.18(\mathrm{~m}, 2 \mathrm{H}), 3.09(\mathrm{~s}, 3 \mathrm{H}) ;{ }^{13} \mathrm{C} \mathrm{NMR}$ $\left(100 \mathrm{MHz}, \mathrm{CDCl}_{3}\right), \delta(\mathrm{ppm})=190.0\left(\mathrm{~d}, J_{\mathrm{P}-\mathrm{C}}=5.9 \mathrm{~Hz}\right), 167.4\left(\mathrm{~d}, J_{\mathrm{P}-\mathrm{C}}=14.8 \mathrm{~Hz}\right)$, $143.5\left(\mathrm{~d}, J_{\mathrm{P}-\mathrm{C}}=9.1 \mathrm{~Hz}\right), 133.5\left(\mathrm{~d}, J_{\mathrm{P}-\mathrm{C}}=10.0 \mathrm{~Hz}\right), 132.0\left(\mathrm{~d}, J_{\mathrm{P}-\mathrm{C}}=2.9 \mathrm{~Hz}\right), 130.2$, $128.8,128.6\left(\mathrm{~d}, J_{\text {P-C }}=12.4 \mathrm{~Hz}\right), 127.8,126.2,125.6\left(\mathrm{~d}, J_{\mathrm{P}-\mathrm{C}}=92.9 \mathrm{~Hz}\right), 72.5\left(\mathrm{~d}, J_{\text {P-C }}\right.$ $=110.5 \mathrm{~Hz}), 49.9 ;{ }^{31} \mathrm{P}$ NMR $\left(162 \mathrm{MHz}, \mathrm{CDCl}_{3}\right), \delta(\mathrm{ppm})=17.6$; HRMS (ESI) Calcd for $\mathrm{C}_{28} \mathrm{H}_{22} \mathrm{ClO}_{3} \mathrm{P}[\mathrm{M}+\mathrm{H}]^{+}=473.1068$, Found $=473.1067$.<smiles>COC(=O)C(C(=O)c1ccccc1Br)=C(P)c1ccccc1</smiles>

Methyl 3-(2-bromophenyl)-3-oxo-2-(triphenylphosphoranylidene)propanoate 25. According to the general procedure A, bromo-2-iodobenzene (141.4 mg, $0.5 \mathrm{mmol}$ ), methyl 2-(triphenylphosphoranylidene)acetate $(267.4 \mathrm{mg}, 0.8 \mathrm{mmol})$, palladium chloride $\mathrm{mg}$, 0.01 mmol), 
(9,9-dimethyl-9H-xanthene-4,5-diyl)bis(diphenylphosphine) (11.5 mg, $0.02 \mathrm{mmol}$ ), Potassium carbonate $(69.1 \mathrm{mg}, 0.5 \mathrm{mmol}), 1$,4-dioxane $(3 \mathrm{~mL}), \mathrm{CO}(1 \mathrm{~atm}), 100{ }^{\circ} \mathrm{C}$, 24 h, compound 25 was synthesized and purified on silica gel with EtOAc:PE (V:V = $\left.1: 2, \mathrm{R}_{\mathrm{f}}=0.15\right)$ to give a colorless solid $(196 \mathrm{mg}, 76 \%), \mathrm{mp}=175-178{ }^{\circ} \mathrm{C} ;{ }^{1} \mathrm{H}$ NMR $\left(400 \mathrm{MHz}, \mathrm{CDCl}_{3}\right), \delta(\mathrm{ppm})=7.85-7.80(\mathrm{~m}, 6 \mathrm{H}), 7.58-7.55(\mathrm{~m}, 3 \mathrm{H}), 7.52-7.48(\mathrm{~m}, 7$ $\mathrm{H})$, 7.31-7.28 (m, $2 \mathrm{H}), 7.15-7.10(\mathrm{~m}, 1 \mathrm{H}), 3.09$ (s ,3 H); ${ }^{13} \mathrm{C}$ NMR (100 MHz, $\left.\mathrm{CDCl}_{3}\right), \delta(\mathrm{ppm})=190.9\left(\mathrm{~d}, J_{\mathrm{P}-\mathrm{C}}=5.9 \mathrm{~Hz}\right), 167.4\left(\mathrm{~d}, J_{\mathrm{P}-\mathrm{C}}=14.7 \mathrm{~Hz}\right), 145.6\left(\mathrm{~d}, J_{\mathrm{P}-\mathrm{C}}\right.$ $=9.4 \mathrm{~Hz}), 133.5\left(\mathrm{~d}, J_{\mathrm{P}-\mathrm{C}}=10.0 \mathrm{~Hz}\right), 132.1\left(\mathrm{~d}, J_{\mathrm{P}-\mathrm{C}}=2.9 \mathrm{~Hz}\right), 132.0,128.7\left(\mathrm{~d}, J_{\mathrm{P}-\mathrm{C}}=\right.$ $12.5 \mathrm{~Hz}), 127.8,126.8,125.5\left(\mathrm{~d}, J_{\mathrm{P}-\mathrm{C}}=92.9 \mathrm{~Hz}\right), 119.2,72.2\left(\mathrm{~d}, J_{\mathrm{P}-\mathrm{C}}=110.2 \mathrm{~Hz}\right)$, 50.0; ${ }^{31} \mathrm{P}$ NMR $\left(162 \mathrm{MHz}, \mathrm{CDCl}_{3}\right), \delta(\mathrm{ppm})=17.6$; HRMS (ESI) Calcd for $\mathrm{C}_{28} \mathrm{H}_{22} \mathrm{BrO}_{3} \mathrm{P}[\mathrm{M}+\mathrm{H}]^{+}=517.0563$, Found $=517.0565$.<smiles>COC(=O)C1=C(C(=O)Oc2ccccc2)C(=O)c2ccccc21</smiles>

Methyl-3-oxo-3-(2-(trifluoromethoxy)phenyl)-2-(triphenylphosphoranylidene)propanoate 26. According to the general procedure A, 1-iodo-2-(trifluoromethoxy)benzene $\quad(144.0 \quad \mathrm{mg}, \quad 0.5 \quad \mathrm{mmol}), \quad$ methyl 2-(triphenylphosphoranylidene)acetate (267.4 mg, $0.8 \mathrm{mmol})$, palladium chloride (1.7 mg, $0.01 \mathrm{mmol})$, (9,9-dimethyl-9H-xanthene-4,5-diyl)bis(diphenylphosphine) (11.5 mg, $0.02 \mathrm{mmol})$, Potassium carbonate $(69.1 \mathrm{mg}, 0.5 \mathrm{mmol})$, 1,4-dioxane (3 mL), CO ( $1 \mathrm{~atm}), 100{ }^{\circ} \mathrm{C}, 12 \mathrm{~h}$, compound 26 was synthesized and purified on silica gel with EtOAc:PE $\left(\mathrm{V}: \mathrm{V}=1: 2, \mathrm{R}_{\mathrm{f}}=0.25\right)$ to give a colorless solid $(204 \mathrm{mg}, 78 \%), \mathrm{mp}=$ $147-150{ }^{\circ} \mathrm{C} ;{ }^{1} \mathrm{H}$ NMR $\left(400 \mathrm{MHz}, \mathrm{CDCl}_{3}\right), \delta(\mathrm{ppm})=7.79-7.74(\mathrm{~m}, 6 \mathrm{H}), 7.58-7.47(\mathrm{~m}$, $9 \mathrm{H})$, 7.39-7.37 (m, $1 \mathrm{H})$, 7.33-7.29 (m, $1 \mathrm{H}), 7.25-7.21(\mathrm{~m}, 2 \mathrm{H}), 3.06(\mathrm{~s}, 3 \mathrm{H}) ;{ }^{13} \mathrm{C}$ $\operatorname{NMR}\left(100 \mathrm{MHz}, \mathrm{CDCl}_{3}\right), \delta(\mathrm{ppm})=188.1\left(\mathrm{~d}, J_{\mathrm{P}-\mathrm{C}}=6.1 \mathrm{~Hz}\right), 167.4\left(\mathrm{~d}, J_{\mathrm{P}-\mathrm{C}}=14.7\right.$ $\mathrm{Hz}), 145.3,137.5\left(\mathrm{~d}, J_{\text {P-C }}=9.2 \mathrm{~Hz}\right), 133.4\left(\mathrm{~d}, J_{\mathrm{P}-\mathrm{C}}=9.7 \mathrm{~Hz}\right), 132.0\left(\mathrm{~d}, J_{\text {P-C }}=2.9\right.$ $\mathrm{Hz}), 128.9,128.6\left(\mathrm{~d}, J_{\mathrm{P}-\mathrm{C}}=12.6 \mathrm{~Hz}\right), 126.0,125.5\left(\mathrm{~d}, J_{\mathrm{P}-\mathrm{C}}=93.1 \mathrm{~Hz}\right), 120.7\left(\mathrm{~d}, J_{\mathrm{F}-\mathrm{C}}\right.$ $=255.9 \mathrm{~Hz}), 119.0,72.8\left(\mathrm{~d}, J_{\mathrm{P}-\mathrm{C}}=110.9 \mathrm{~Hz}\right), 49.8 ;{ }^{31} \mathrm{P} \mathrm{NMR}\left(162 \mathrm{MHz}, \mathrm{CDCl}_{3}\right), \delta$ $(\mathrm{ppm})=17.5$; HRMS (ESI) Calcd for $\mathrm{C}_{29} \mathrm{H}_{22} \mathrm{~F}_{3} \mathrm{O}_{4} \mathrm{P}[\mathrm{M}+\mathrm{H}]^{+}=523.1281$, Found $=$ 523.1276. 
<smiles>COC(=O)C(=P)C(=O)c1ccccc1C</smiles>

Methyl 3-oxo-3-(o-tolyl)-2-(triphenylphosphoranylidene)propanoate

27.

According to the general procedure A, 1-iodo-2-methylbenzene (109.0 mg, $0.5 \mathrm{mmol})$, methyl 2-(triphenylphosphoranylidene)acetate (267.4 mg, $0.8 \mathrm{mmol})$, palladium chloride

$\mathrm{mg}$, 0.01 mmol), (9,9-dimethyl-9H-xanthene-4,5-diyl)bis(diphenylphosphine) (11.5 mg, $0.02 \mathrm{mmol}$ ), Potassium carbonate $(69.1 \mathrm{mg}, 0.5 \mathrm{mmol}), 1$,4-dioxane $(3 \mathrm{~mL}), \mathrm{CO}(1 \mathrm{~atm}), 100{ }^{\circ} \mathrm{C}$, $12 \mathrm{~h}$, compound 27 was synthesized and purified on silica gel with EtOAc:PE (V:V = $\left.1: 2, \mathrm{R}_{\mathrm{f}}=0.26\right)$ to give a pale yellow solid $(45 \mathrm{mg}, 20 \%), \mathrm{mp}=165-167{ }^{\circ} \mathrm{C} ;{ }^{1} \mathrm{H} \mathrm{NMR}$ $\left(400 \mathrm{MHz}, \mathrm{CDCl}_{3}\right), \delta(\mathrm{ppm})=7.79-7.74(\mathrm{~m}, 6 \mathrm{H})$, 7.58-7.49 (m, $\left.9 \mathrm{H}\right)$, 7.29-7.27 (m,1 $\mathrm{H})$, 7.15-7.10 (m, $3 \mathrm{H}), 3.04(\mathrm{~s}, 3 \mathrm{H}), 2.38(\mathrm{~s}, 3 \mathrm{H}) ;{ }^{13} \mathrm{C} \mathrm{NMR}\left(100 \mathrm{MHz}, \mathrm{CDCl}_{3}\right), \delta$ $(\mathrm{ppm})=194.2\left(\mathrm{~d}, J_{\mathrm{P}-\mathrm{C}}=5.0 \mathrm{~Hz}\right), 167.6\left(\mathrm{~d}, J_{\mathrm{P}-\mathrm{C}}=15.4 \mathrm{~Hz}\right), 143.9\left(\mathrm{~d}, J_{\mathrm{P}-\mathrm{C}}=8.7 \mathrm{~Hz}\right)$, $134.1,133.4\left(\mathrm{~d}, J_{\text {P-C }}=9.7 \mathrm{~Hz}\right), 131.9\left(\mathrm{~d}, J_{\text {P-C }}=2.8 \mathrm{~Hz}\right), 129.7,128.6\left(\mathrm{~d}, J_{\text {P-C }}=12.5\right.$ $\mathrm{Hz}), 127.6,126.3,126.0\left(\mathrm{~d}, J_{\mathrm{P}-\mathrm{C}}=92.8 \mathrm{~Hz}\right), 124.8,72.3\left(\mathrm{~d}, J_{\mathrm{P}-\mathrm{C}}=109.4 \mathrm{~Hz}\right), 49.8$, 19.5; ${ }^{31} \mathrm{P}$ NMR $\left(162 \mathrm{MHz}, \mathrm{CDCl}_{3}\right), \delta(\mathrm{ppm})=17.6$; HRMS (ESI) Calcd for $\mathrm{C}_{29} \mathrm{H}_{25} \mathrm{O}_{3} \mathrm{P}[\mathrm{M}+\mathrm{H}]^{+}=453.1614$, Found $=453.1610$.<smiles>COC(=O)C(=P)C(=O)c1ccccc1OC</smiles>

Methyl 3-(2-methoxyphenyl)-3-oxo-2-(triphenylphosphoranylidene)propanoate 28. According to the general procedure A, 1-iodo-2-methoxybenzene (117.0 mg, 0.5 mmol), methyl 2-(triphenylphosphoranylidene)acetate (267.4 mg, $0.8 \mathrm{mmol}$ ),

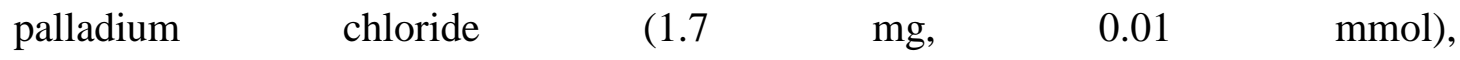
(9,9-dimethyl-9H-xanthene-4,5-diyl)bis(diphenylphosphine) (11.5 mg, $0.02 \mathrm{mmol}$ ), Potassium carbonate $(69.1 \mathrm{mg}, 0.5 \mathrm{mmol}), 1,4$-dioxane $(3 \mathrm{~mL}), \mathrm{CO}(1 \mathrm{~atm}), 100{ }^{\circ} \mathrm{C}$, $12 \mathrm{~h}$, compound 28 was synthesized and purified on silica gel with EtOAc:PE (V:V = $\left.1: 2, \mathrm{R}_{\mathrm{f}}=0.10\right)$ to give a colorless solid $(70 \mathrm{mg}, 30 \%), \mathrm{mp}=175-178{ }^{\circ} \mathrm{C} ;{ }^{1} \mathrm{H} \mathrm{NMR}$ $\left(400 \mathrm{MHz}, \mathrm{CDCl}_{3}\right), \delta(\mathrm{ppm})=7.80-7.75(\mathrm{~m}, 6 \mathrm{H}), 7.56-7.45(\mathrm{~m}, 9 \mathrm{H}), 7.28-7.22(\mathrm{~m}, 2$ 
$\mathrm{H}), 6.93(\mathrm{t}, J=7.4 \mathrm{~Hz}, 1 \mathrm{H}), 6.86(\mathrm{~d}, J=8.2 \mathrm{~Hz}, 1 \mathrm{H}), 3.87(\mathrm{~s}, 3 \mathrm{H}), 3.07(\mathrm{~s}, 3 \mathrm{H}) ;{ }^{13} \mathrm{C}$ NMR $\left(100 \mathrm{MHz}, \mathrm{CDCl}_{3}\right), \delta(\mathrm{ppm})=190.9\left(\mathrm{~d}, J_{\mathrm{P}-\mathrm{C}}=5.5 \mathrm{~Hz}\right), 167.8\left(\mathrm{~d}, J_{\text {P-C }}=16.0\right.$ $\mathrm{Hz}), 156.0,134.2\left(\mathrm{~d}, J_{\mathrm{P}-\mathrm{C}}=8.4 \mathrm{~Hz}\right), 133.5\left(\mathrm{~d}, J_{\mathrm{P}-\mathrm{C}}=9.9 \mathrm{~Hz}\right), 131.7\left(\mathrm{~d}, J_{\mathrm{P}-\mathrm{C}}=2.9\right.$ $\mathrm{Hz}), 129.0,128.5\left(\mathrm{~d}, J_{\mathrm{P}-\mathrm{C}}=12.5 \mathrm{~Hz}\right), 127.6,126.3\left(\mathrm{~d}, J_{\mathrm{P}-\mathrm{C}}=93.1 \mathrm{~Hz}\right), 120.3,110.5$, $72.3\left(\mathrm{~d}, J_{\mathrm{P}-\mathrm{C}}=112.0 \mathrm{~Hz}\right), 55.8,49.7 ;{ }^{31} \mathrm{P} \mathrm{NMR}\left(162 \mathrm{MHz}, \mathrm{CDCl}_{3}\right), \delta(\mathrm{ppm})=17.4$; HRMS (ESI) Calcd for $\mathrm{C}_{29} \mathrm{H}_{25} \mathrm{O}_{4} \mathrm{P}[\mathrm{M}+\mathrm{H}]^{+}=469.1563$, Found $=469.1565$.<smiles>COC(=O)C(=P)C(=O)c1ccc(F)c(F)c1</smiles>

Methyl 3-(3,4-difluorophenyl)-3-oxo-2-(triphenylphosphoranylidene)propanoate 29. According to the general procedure A, 1,2-difluoro-4-iodobenzene (120.0 mg, 0.5 mmol), methyl 2-(triphenylphosphoranylidene)acetate (200.6 mg, $0.6 \mathrm{mmol}$ ), palladium chloride $\quad(1.7 \quad \mathrm{mg}, \quad 0.01 \quad \mathrm{mmol})$, (9,9-dimethyl-9H-xanthene-4,5-diyl)bis(diphenylphosphine) (11.5 mg, $0.02 \mathrm{mmol}$ ), Potassium carbonate (69.1 mg, $0.5 \mathrm{mmol}), 1,4$-dioxane $(3 \mathrm{~mL}), \mathrm{CO}(1 \mathrm{~atm}), 100{ }^{\circ} \mathrm{C}$, $12 \mathrm{~h}$, compound 29 was synthesized and purified on silica gel with EtOAc:PE (V:V = $\left.1: 2, \mathrm{R}_{\mathrm{f}}=0.31\right)$ to give a colorless solid $(221 \mathrm{mg}, 93 \%), \mathrm{mp}=162-165{ }^{\circ} \mathrm{C} ;{ }^{1} \mathrm{H} \mathrm{NMR}$ $\left(400 \mathrm{MHz}, \mathrm{CDCl}_{3}\right), \delta(\mathrm{ppm})=7.77-7.72(\mathrm{~m}, 6 \mathrm{H}), 7.59-7.55(\mathrm{~m}, 3 \mathrm{H}), 7.53-7.45(\mathrm{~m}, 8$ H), 7.15-7.08 (m, $1 \mathrm{H}), 3.16(\mathrm{~s}, 3 \mathrm{H}) ;{ }^{13} \mathrm{C} \mathrm{NMR}(100 \mathrm{MHz}, \mathrm{CDCl} 3), \delta(\mathrm{ppm})=190.6$ $\left(\mathrm{d}, J_{\mathrm{P}-\mathrm{C}}=4.8 \mathrm{~Hz}\right), 167.8\left(\mathrm{~d}, J_{\mathrm{P}-\mathrm{C}}=13.7 \mathrm{~Hz}\right), 151.3\left(\mathrm{dd}, J_{\mathrm{F}-\mathrm{C}}=12.9 \mathrm{~Hz}, J_{\mathrm{F}-\mathrm{C}}=248.6\right.$ $\mathrm{Hz}), 149.5\left(\mathrm{dd}, J_{\text {F-C }}=12.8 \mathrm{~Hz}, J_{\text {F-C }}=245.9 \mathrm{~Hz}\right), 140.0-139.8(\mathrm{~m}), 133.3\left(\mathrm{~d}, J_{\text {P-C }}=\right.$ $9.7 \mathrm{~Hz}), 132.1\left(\mathrm{~d}, J_{\mathrm{P}-\mathrm{C}}=2.9 \mathrm{~Hz}\right), 128.7\left(\mathrm{~d}, J_{\mathrm{P}-\mathrm{C}}=12.4 \mathrm{~Hz}\right), 125.7\left(\mathrm{~d}, J_{\mathrm{P}-\mathrm{C}}=92.9 \mathrm{~Hz}\right)$, 124.9-124.8 (m), $117.7\left(\mathrm{~d}, J_{\mathrm{F}-\mathrm{C}}=17.7 \mathrm{~Hz}\right), 116.0\left(\mathrm{~d}, J_{\mathrm{F}-\mathrm{C}}=17.4 \mathrm{~Hz}\right), 69.3\left(\mathrm{~d}, J_{\mathrm{P}-\mathrm{C}}=\right.$ $110.3 \mathrm{~Hz}), 49.8 ;{ }^{31} \mathrm{P} \mathrm{NMR}\left(162 \mathrm{MHz}, \mathrm{CDCl}_{3}\right), \delta(\mathrm{ppm})=19.2$; HRMS (ESI) Calcd for $\mathrm{C}_{28} \mathrm{H}_{21} \mathrm{~F}_{2} \mathrm{O}_{3} \mathrm{P}[\mathrm{M}+\mathrm{H}]^{+}=475.1269$, Found $=475.1270$.<smiles>COC(=O)C(=P)C(=O)c1cc(C(F)(F)F)cc(C(F)(F)F)c1</smiles>

Methyl-3-(3,5-bis(trifluoromethyl)phenyl)-3-oxo-2-(triphenylphosphoranylidene) propanoate 30. According to the general procedure A, 
1-iodo-3,5-bis(trifluoromethyl)benzene $\quad(170.0 \quad \mathrm{mg}, \quad 0.5 \quad \mathrm{mmol})$, methyl 2-(triphenylphosphoranylidene)acetate (200.6 mg, $0.6 \mathrm{mmol})$, palladium chloride (1.7 mg, $0.01 \mathrm{mmol})$, (9,9-dimethyl-9H-xanthene-4,5-diyl)bis(diphenylphosphine) (11.5 $\mathrm{mg}, 0.02 \mathrm{mmol})$, Potassium carbonate $(69.1 \mathrm{mg}, 0.5 \mathrm{mmol})$, 1,4-dioxane $(3 \mathrm{~mL}), \mathrm{CO}$ ( $1 \mathrm{~atm}), 100{ }^{\circ} \mathrm{C}, 12 \mathrm{~h}$, compound 30 was synthesized and purified on silica gel with EtOAc:PE $\left(\mathrm{V}: \mathrm{V}=1: 2, \mathrm{R}_{\mathrm{f}}=0.42\right)$ to give a pale yellow solid $(256 \mathrm{mg}, 89 \%), \mathrm{mp}=$ 163-165 ${ }^{\circ} \mathrm{C} ;{ }^{1} \mathrm{H}$ NMR $\left(400 \mathrm{MHz}, \mathrm{CDCl}_{3}\right), \delta(\mathrm{ppm})=8.09(\mathrm{~s}, 2 \mathrm{H}), 7.86(\mathrm{~s}, 1 \mathrm{H})$, 7.79-7.74 (m, $6 \mathrm{H}), 7.61-7.58(\mathrm{~m}, 3 \mathrm{H}), 7.55-7.50(\mathrm{~m}, 6 \mathrm{H}), 3.15(\mathrm{~s}, 3 \mathrm{H}) ;{ }^{13} \mathrm{C} \mathrm{NMR}$ $\left(100 \mathrm{MHz}, \mathrm{CDCl}_{3}\right), \delta(\mathrm{ppm})=189.5\left(\mathrm{~d}, J_{\mathrm{P}-\mathrm{C}}=5.9 \mathrm{~Hz}\right), 167.4\left(\mathrm{~d}, J_{\mathrm{P}-\mathrm{C}}=14.1 \mathrm{~Hz}\right)$, $144.7\left(\mathrm{~d}, J_{\mathrm{P}-\mathrm{C}}=9.7 \mathrm{~Hz}\right), 133.4\left(\mathrm{~d}, J_{\mathrm{P}-\mathrm{C}}=9.7 \mathrm{~Hz}\right), 132.3\left(\mathrm{~d}, J_{\mathrm{P}-\mathrm{C}}=2.9 \mathrm{~Hz}\right), 130.4(\mathrm{q}$, $\left.J_{\text {F-C }}=32.9 \mathrm{~Hz}\right), 128.8\left(\mathrm{~d}, J_{\mathrm{P}-\mathrm{C}}=12.5 \mathrm{~Hz}\right), 125.5\left(\mathrm{~d}, J_{\mathrm{P}-\mathrm{C}}=93.1 \mathrm{~Hz}\right), 123.5\left(\mathrm{~d}, J_{\mathrm{F}-\mathrm{C}}=\right.$ 271.0 Hz), 122.7, $70.7\left(\mathrm{~d}, J_{\mathrm{P}-\mathrm{C}}=110.4 \mathrm{~Hz}\right), 49.9 ;{ }^{31} \mathrm{P}$ NMR $\left(162 \mathrm{MHz}, \mathrm{CDCl}_{3}\right), \delta$ $(\mathrm{ppm})=19.1$; HRMS (ESI) Calcd for $\mathrm{C}_{30} \mathrm{H}_{21} \mathrm{~F}_{6} \mathrm{O}_{3} \mathrm{P}[\mathrm{M}+\mathrm{H}]^{+}=575.1205$, Found $=$ 575.1200.

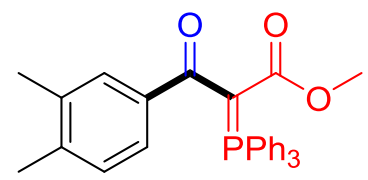

Methyl 3-(3,4-dimethylphenyl)-3-oxo-2-(triphenylphosphoranylidene)propanoate 31. According to the general procedure A, 4-iodo-1,2-dimethylbenzene (116.0 mg, 0.5 mmol), methyl 2-(triphenylphosphoranylidene)acetate $(334.3 \mathrm{mg}, 1.0 \mathrm{mmol}$ ),

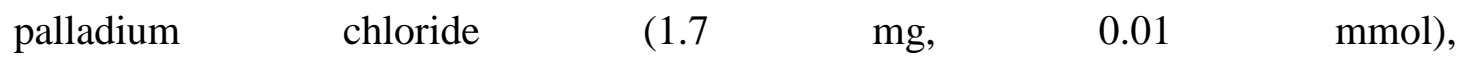
(9,9-dimethyl-9H-xanthene-4,5-diyl)bis(diphenylphosphine) (11.5 mg, $0.02 \mathrm{mmol}$ ), Potassium carbonate $(69.1 \mathrm{mg}, 0.5 \mathrm{mmol}), 1$,4-dioxane $(3 \mathrm{~mL}), \mathrm{CO}(1 \mathrm{~atm}), 100{ }^{\circ} \mathrm{C}$, $24 \mathrm{~h}$, compound 31 was synthesized and purified on silica gel with EtOAc:PE (V:V = $\left.1: 2, \mathrm{R}_{\mathrm{f}}=0.17\right)$ to give a yellow solid $(138 \mathrm{mg}, 59 \%), \mathrm{mp}=133-135{ }^{\circ} \mathrm{C} ;{ }^{1} \mathrm{H}$ NMR $\left(400 \mathrm{MHz}, \mathrm{CDCl}_{3}\right), \delta(\mathrm{ppm})=7.78-7.73(\mathrm{~m}, 6 \mathrm{H}), 7.55-7.44(\mathrm{~m}, 11 \mathrm{H}), 7.11(\mathrm{~d}, J=$ $7.7 \mathrm{~Hz}, 1 \mathrm{H}), 3.16(\mathrm{~s}, 3 \mathrm{H}), 2.26(\mathrm{~s}, 6 \mathrm{H}) ;{ }^{13} \mathrm{C} \mathrm{NMR}\left(100 \mathrm{MHz}, \mathrm{CDCl}_{3}\right), \delta(\mathrm{ppm})=$ $193.3\left(\mathrm{~d}, J_{\mathrm{P}-\mathrm{C}}=5.2 \mathrm{~Hz}\right), 168.1\left(\mathrm{~d}, J_{\mathrm{P}-\mathrm{C}}=14.6 \mathrm{~Hz}\right), 140.5\left(\mathrm{~d}, J_{\mathrm{P}-\mathrm{C}}=8.8 \mathrm{~Hz}\right), 138.4$, $135.4,133.4\left(\mathrm{~d}, J_{\text {P-C }}=9.7 \mathrm{~Hz}\right), 131.8\left(\mathrm{~d}, J_{\text {P-C }}=2.8 \mathrm{~Hz}\right), 129.5,128.6\left(\mathrm{~d}, J_{\text {P-C }}=12.4\right.$ $\mathrm{Hz}), 128.5,126.4(\mathrm{~d}, J$ P-C $=92.7 \mathrm{~Hz}), 126.3,68.8(\mathrm{~d}, J$ P-C $=110.9 \mathrm{~Hz}), 49.7$, 
19.9,19.8; ${ }^{31} \mathrm{P}$ NMR (162 MHz, $\left.\mathrm{CDCl}_{3}\right), \delta$ (ppm) = 19.0; HRMS (ESI) Calcd for $\mathrm{C}_{30} \mathrm{H}_{27} \mathrm{O}_{3} \mathrm{P}[\mathrm{M}+\mathrm{H}]^{+}=467.1771$, Found $=467.1770$.

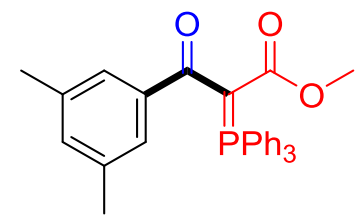

\section{Methyl 3-(3,5-dimethylphenyl)-3-oxo-2-(triphenylphosphoranylidene)propanoate}

32. According to the general procedure A, 1-iodo-3,5-dimethylbenzene (116.0 mg, 0.5 mmol), methyl 2-(triphenylphosphoranylidene)acetate $(267.4 \mathrm{mg}, 0.8 \mathrm{mmol}$ ), palladium chloride $\quad\left(\begin{array}{llll}1.7 & \mathrm{mg}, & 0.01 & \mathrm{mmol}) \text {, }\end{array}\right.$ (9,9-dimethyl-9H-xanthene-4,5-diyl)bis(diphenylphosphine) (11.5 mg, $0.02 \mathrm{mmol}$ ), Potassium carbonate (69.1 mg, $0.5 \mathrm{mmol}), 1$,4-dioxane $(3 \mathrm{~mL}), \mathrm{CO}(1 \mathrm{~atm}), 100{ }^{\circ} \mathrm{C}$, $24 \mathrm{~h}$, compound 32 was synthesized and purified on silica gel with EtOAc:PE (V:V = $\left.1: 2, \mathrm{R}_{\mathrm{f}}=0.22\right)$ to give a pale yellow solid $(191 \mathrm{mg}, 82 \%), \mathrm{mp}=133-136{ }^{\circ} \mathrm{C} ;{ }^{1} \mathrm{H}$ NMR $\left(400 \mathrm{MHz}, \mathrm{CDCl}_{3}\right), \delta(\mathrm{ppm})=7.80-7.74(\mathrm{~m}, 6 \mathrm{H}), 7.56-7.52(\mathrm{~m}, 3 \mathrm{H})$, 7.50-7.45 (m, $6 \mathrm{H}), 7.31$ (s, $2 \mathrm{H}), 7.01(\mathrm{~s}, 1 \mathrm{H}), 3.18$ (s, $3 \mathrm{H}), 2.34(\mathrm{~s}, 6 \mathrm{H}) ;{ }^{13} \mathrm{C} \mathrm{NMR}$ $\left(100 \mathrm{MHz}, \mathrm{CDCl}_{3}\right), \delta(\mathrm{ppm})=193.6\left(\mathrm{~d}, J_{\mathrm{P}-\mathrm{C}}=4.9 \mathrm{~Hz}\right), 168.2\left(\mathrm{~d}, J_{\mathrm{P}-\mathrm{C}}=15.0 \mathrm{~Hz}\right)$, $143.0\left(\mathrm{~d}, J_{\mathrm{P}-\mathrm{C}}=8.6 \mathrm{~Hz}\right), 136.6,133.4\left(\mathrm{~d}, J_{\mathrm{P}-\mathrm{C}}=9.8 \mathrm{~Hz}\right), 131.9\left(\mathrm{~d}, J_{\mathrm{P}-\mathrm{C}}=2.9 \mathrm{~Hz}\right)$, 131.3, $128.6\left(\mathrm{~d}, J_{\text {P-C }}=12.4 \mathrm{~Hz}\right), 126.2\left(\mathrm{~d}, J_{\mathrm{P}-\mathrm{C}}=92.8 \mathrm{~Hz}\right), 126.0,69.2\left(\mathrm{~d}, J_{\text {P-C }}=\right.$ 111.1 Hz), 49.8, 21.4; ${ }^{31} \mathrm{P}$ NMR (162 $\left.\mathrm{MHz}, \mathrm{CDCl}_{3}\right), \delta(\mathrm{ppm})=18.8$; HRMS (ESI) Calcd for $\mathrm{C}_{30} \mathrm{H}_{27} \mathrm{O}_{3} \mathrm{P}[\mathrm{M}+\mathrm{H}]^{+}=467.1771$, Found $=467.1774$.<smiles>COC(=O)C(=P)C(=O)c1cccc2ccccc12</smiles>

Methyl 3-(naphthalen-1-yl)-3-oxo-2-(triphenylphosphoranylidene)propanoate 33. According to the general procedure A, 1-iodonaphthalene (127.0 mg, $0.5 \mathrm{mmol})$, methyl 2-(triphenylphosphoranylidene)acetate $(267.4 \mathrm{mg}, 0.8 \mathrm{mmol})$, palladium chloride $\mathrm{mg}$, 0.01 mmol), (9,9-dimethyl-9H-xanthene-4,5-diyl)bis(diphenylphosphine) (11.5 mg, $0.02 \mathrm{mmol}$ ), Potassium carbonate $(69.1 \mathrm{mg}, 0.5 \mathrm{mmol}), 1$,4-dioxane $(3 \mathrm{~mL}), \mathrm{CO}(1 \mathrm{~atm}), 100{ }^{\circ} \mathrm{C}$, $24 \mathrm{~h}$, compound 33 was synthesized and purified on silica gel with EtOAc:PE (V:V = 
$\left.1: 2, \mathrm{R}_{\mathrm{f}}=0.17\right)$ to give a pale yellow solid $(93 \mathrm{mg}, 38 \%), \mathrm{mp}=168-172{ }^{\circ} \mathrm{C} ;{ }^{1} \mathrm{H} \mathrm{NMR}$ $\left(400 \mathrm{MHz}, \mathrm{CDCl}_{3}\right), \delta(\mathrm{ppm})=8.13-8.11(\mathrm{~m}, 1 \mathrm{H}), 7.87-7.77(\mathrm{~m}, 8 \mathrm{H})$, 7.61-7.50 (m, $10 \mathrm{H}), 7.47-7.39(\mathrm{~m}, 3 \mathrm{H}), 2.91(\mathrm{~s}, 3 \mathrm{H}) ;{ }^{13} \mathrm{C} \mathrm{NMR}\left(100 \mathrm{MHz}, \mathrm{CDCl}_{3}\right), \delta(\mathrm{ppm})=$ $193.1\left(\mathrm{~d}, J_{\mathrm{P}-\mathrm{C}}=5.1 \mathrm{~Hz}\right), 167.7\left(\mathrm{~d}, J_{\mathrm{P}-\mathrm{C}}=15.7 \mathrm{~Hz}\right), 141.8\left(\mathrm{~d}, J_{\mathrm{P}-\mathrm{C}}=8.9 \mathrm{~Hz}\right), 133.4(\mathrm{~d}$, $\left.J_{\text {P-C }}=10.0 \mathrm{~Hz}\right), 132.0\left(\mathrm{~d}, J_{\text {P-C }}=2.9 \mathrm{~Hz}\right), 130.7,128.7\left(\mathrm{~d}, J_{\text {P-C }}=12.4 \mathrm{~Hz}\right), 128.2$, $128.0,126.0\left(\mathrm{~d}, J_{\text {P-C }}=93.0 \mathrm{~Hz}\right), 125.8,125.7,125.3,124.7,124.3,73.1\left(\mathrm{~d}, J_{\text {P-C }}=\right.$ $110.1 \mathrm{~Hz}), 49.8 ;{ }^{31} \mathrm{P}$ NMR $\left(162 \mathrm{MHz}, \mathrm{CDCl}_{3}\right), \delta(\mathrm{ppm})=17.9$; HRMS (ESI) Calcd for $\mathrm{C}_{32} \mathrm{H}_{25} \mathrm{O}_{3} \mathrm{P}[\mathrm{M}+\mathrm{H}]^{+}=489.1614$, Found $=489.1615$.

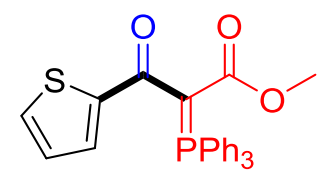

\section{Methyl 3-oxo-3-(thiophen-2-yl)-2-(triphenylphosphoranylidene)propanoate 34.}

According to the general procedure A, 2-iodothiophene (105.0 mg, $0.5 \mathrm{mmol})$, methyl 2-(triphenylphosphoranylidene)acetate $(267.4 \mathrm{mg}, 0.8 \mathrm{mmol})$, palladium chloride (1.7 mg, $0.01 \mathrm{mmol}),(9,9$-dimethyl-9H-xanthene-4,5-diyl)bis(diphenylphosphine) (11.5 mg, $0.02 \mathrm{mmol})$, Potassium carbonate $(69.1 \mathrm{mg}, 0.5 \mathrm{mmol})$, 1,4-dioxane $(3 \mathrm{~mL}), \mathrm{CO}$ (1 atm), $100{ }^{\circ} \mathrm{C}, 24 \mathrm{~h}$, compound $\mathbf{3 4}$ was synthesized and purified on silica gel with EtOAc:PE $\left(\mathrm{V}: \mathrm{V}=1: 2, \mathrm{R}_{\mathrm{f}}=0.21\right)$ to give a pale yellow solid $(107 \mathrm{mg}, 48 \%), \mathrm{mp}=$ 141-145 ${ }^{\circ} \mathrm{C} ;{ }^{1} \mathrm{H}$ NMR $\left(400 \mathrm{MHz}, \mathrm{CDCl}_{3}\right), \delta(\mathrm{ppm})=7.92(\mathrm{~d}, J=3.5 \mathrm{~Hz}, 1 \mathrm{H})$, 7.77-7.72 (m, $6 \mathrm{H})$, 7.54-7.50 (m, $3 \mathrm{H}), 7.48-7.43$ (m, $6 \mathrm{H}), 7.38$ (d, J=4.8 Hz, $1 \mathrm{H})$, $7.04(\mathrm{t}, J=4.7 \mathrm{~Hz}, 1 \mathrm{H}), 3.21(\mathrm{~s}, 3 \mathrm{H}) ;{ }^{13} \mathrm{C} \mathrm{NMR}\left(100 \mathrm{MHz}, \mathrm{CDCl}_{3}\right), \delta(\mathrm{ppm})=182.7$ $\left(\mathrm{d}, J_{\mathrm{P}-\mathrm{C}}=5.8 \mathrm{~Hz}\right), 167.6\left(\mathrm{~d}, J_{\mathrm{P}-\mathrm{C}}=14.0 \mathrm{~Hz}\right), 146.4\left(\mathrm{~d}, J_{\mathrm{P}-\mathrm{C}}=10.6 \mathrm{~Hz}\right), 133.3\left(\mathrm{~d}, J_{\mathrm{P}-\mathrm{C}}\right.$ $=9.7 \mathrm{~Hz}), 131.8\left(\mathrm{~d}, J_{\mathrm{P}-\mathrm{C}}=2.8 \mathrm{~Hz}\right), 130.7,129.2,128.6\left(\mathrm{~d}, J_{\mathrm{P}-\mathrm{C}}=12.4 \mathrm{~Hz}\right), 126.7$, $126.3\left(\mathrm{~d}, J_{\mathrm{P}-\mathrm{C}}=93.1 \mathrm{~Hz}\right), 69.7\left(\mathrm{~d}, J_{\mathrm{P}-\mathrm{C}}=112.3 \mathrm{~Hz}\right), 49.9 ;{ }^{31} \mathrm{P}$ NMR $(162 \mathrm{MHz}$, $\left.\mathrm{CDCl}_{3}\right), \delta(\mathrm{ppm})=19.1$; HRMS (ESI) Calcd for $\mathrm{C}_{26} \mathrm{H}_{21} \mathrm{O}_{3} \mathrm{PS}[\mathrm{M}+\mathrm{H}]^{+}=445.1022$, Found $=445.1028$.<smiles>COC(=O)C(=P)C(=O)c1ccc(Br)nc1</smiles>

Methyl-3-(6-bromopyridin-3-yl)-3-oxo-2-(triphenylphosphoranylidene)propanoa 
-te 35. According to the general procedure A, 2-bromo-5-iodopyridine (114.9 mg, 0.5 mmol), methyl 2-(triphenylphosphoranylidene)acetate $(334.3 \mathrm{mg}, 1.0 \mathrm{mmol})$, palladium chloride $\quad\left(\begin{array}{llll}1.7 & \mathrm{mg}, & 0.01 & \mathrm{mmol}) \text {, }\end{array}\right.$ (9,9-dimethyl-9H-xanthene-4,5-diyl)bis(diphenylphosphine) (11.5 mg, $0.02 \mathrm{mmol}$ ), Potassium carbonate $(69.1 \mathrm{mg}, 0.5 \mathrm{mmol}), 1,4$-dioxane $(3 \mathrm{~mL}), \mathrm{CO}(1 \mathrm{~atm}), 100{ }^{\circ} \mathrm{C}$, $24 \mathrm{~h}$, compound 35 was synthesized and purified on silica gel with EtOAc:PE (V:V = $\left.1: 2, \mathrm{R}_{\mathrm{f}}=0.16\right)$ to give a pale yellow solid $(155 \mathrm{mg}, 60 \%), \mathrm{mp}=193-195{ }^{\circ} \mathrm{C} ;{ }^{1} \mathrm{H}$ NMR $\left(400 \mathrm{MHz}, \mathrm{CDCl}_{3}\right), \delta(\mathrm{ppm})=8.62(\mathrm{~d}, J=2.3 \mathrm{~Hz}, 1 \mathrm{H}), 7.79(\mathrm{dd}, J=8.2 \mathrm{~Hz}, J$ $=2.4 \mathrm{~Hz}, 1 \mathrm{H}), 7.76-7.71(\mathrm{~m}, 6 \mathrm{H}), 7.60-7.56(\mathrm{~m}, 3 \mathrm{H}), 7.52-7.47(\mathrm{~m}, 6 \mathrm{H}), 7.44(\mathrm{~d}, J$ $=8.2 \mathrm{~Hz}, 1 \mathrm{H}), 3.16(\mathrm{~s}, 3 \mathrm{H}) ;{ }^{13} \mathrm{C} \mathrm{NMR}\left(100 \mathrm{MHz}, \mathrm{CDCl}_{3}\right), \delta(\mathrm{ppm})=189.1\left(\mathrm{~d}, J_{\mathrm{P}-\mathrm{C}}\right.$ $=5.9 \mathrm{~Hz}), 167.6\left(\mathrm{~d}, J_{\text {P-C }}=13.7 \mathrm{~Hz}\right), 150.0,142.5,138.4,137.5\left(\mathrm{~d}, J_{\text {P-C }}=9.4 \mathrm{~Hz}\right)$, $133.3\left(\mathrm{~d}, J_{\mathrm{P}-\mathrm{C}}=9.9 \mathrm{~Hz}\right), 132.2\left(\mathrm{~d}, J_{\mathrm{P}-\mathrm{C}}=2.8 \mathrm{~Hz}\right), 128.8\left(\mathrm{~d}, J_{\mathrm{P}-\mathrm{C}}=12.6 \mathrm{~Hz}\right), 126.8$, $125.3\left(\mathrm{~d}, J_{\mathrm{P}-\mathrm{C}}=92.8 \mathrm{~Hz}\right), 70.6\left(\mathrm{~d}, J_{\mathrm{P}-\mathrm{C}}=110.2 \mathrm{~Hz}\right), 50.0 ;{ }^{31} \mathrm{P}$ NMR $(162 \mathrm{MHz}$, $\left.\mathrm{CDCl}_{3}\right), \delta(\mathrm{ppm})=19.0 ;$ HRMS (ESI) Calcd for $\mathrm{C}_{27} \mathrm{H}_{21} \mathrm{BrNO}_{3} \mathrm{P}[\mathrm{M}+\mathrm{H}]^{+}=518.0515$, Found $=518.0516$.<smiles>COC(=O)C(=P)C(=O)c1ccc(Cl)nc1</smiles>

Methyl-3-(6-chloropyridin-3-yl)-3-oxo-2-(triphenylphosphoranylidene)propanoat -e 36. According to the general procedure A, 2-chloro-5-iodopyridine (119.7 mg, 0.5 mmol), methyl 2-(triphenylphosphoranylidene)acetate (267.4 mg, $0.8 \mathrm{mmol}$ ), palladium chloride $\quad(1.7 \quad \mathrm{mg}, \quad 0.01 \quad \mathrm{mmol})$, (9,9-dimethyl-9H-xanthene-4,5-diyl)bis(diphenylphosphine) (11.5 mg, $0.02 \mathrm{mmol}$ ), Potassium carbonate $(69.1 \mathrm{mg}, 0.5 \mathrm{mmol}), 1$,4-dioxane $(3 \mathrm{~mL}), \mathrm{CO}(1 \mathrm{~atm}), 100{ }^{\circ} \mathrm{C}$, $24 \mathrm{~h}$, compound 36 was synthesized and purified on silica gel with EtOAc:PE (V:V = $\left.1: 2, \mathrm{R}_{\mathrm{f}}=0.15\right)$ to give a colorless solid $(227 \mathrm{mg}, 96 \%), \mathrm{mp}=176-179{ }^{\circ} \mathrm{C} ;{ }^{1} \mathrm{H} \mathrm{NMR}$ $\left(400 \mathrm{MHz}, \mathrm{CDCl}_{3}\right), \delta(\mathrm{ppm})=8.66(\mathrm{~s}, 1 \mathrm{H}), 7.91(\mathrm{dd}, J=8.2 \mathrm{~Hz}, J=2.2 \mathrm{~Hz}, 1 \mathrm{H})$, 7.76-7.71 (m, $6 \mathrm{H}), 7.59-7.55$ (m, $3 \mathrm{H}), 7.52-7.47$ (m, $6 \mathrm{H}), 7.28$ (d, J= 8.2 Hz, $1 \mathrm{H})$, $3.16(\mathrm{~s}, 3 \mathrm{H}),{ }^{13} \mathrm{C} \mathrm{NMR}\left(100 \mathrm{MHz}, \mathrm{CDCl}_{3}\right), \delta(\mathrm{ppm})=189.0\left(\mathrm{~d}, J_{\mathrm{P}-\mathrm{C}}=5.9 \mathrm{~Hz}\right), 167.6$ $\left(\mathrm{d}, J_{\text {P-C }}=13.7 \mathrm{~Hz}\right), 151.6,149.6,138.7,137.2\left(\mathrm{~d}, J_{\mathrm{P}-\mathrm{C}}=9.4 \mathrm{~Hz}\right), 133.3\left(\mathrm{~d}, J_{\text {P-C }}=9.7\right.$ 
$\mathrm{Hz}), 132.2\left(\mathrm{~d}, J_{\mathrm{P}-\mathrm{C}}=2.9 \mathrm{~Hz}\right), 128.8\left(\mathrm{~d}, J_{\mathrm{P}-\mathrm{C}}=12.5 \mathrm{~Hz}\right), 125 . .3\left(\mathrm{~d}, J_{\mathrm{P}-\mathrm{C}}=92.9 \mathrm{~Hz}\right)$, $122.9,70.5\left(\mathrm{~d}, J_{\mathrm{P}-\mathrm{C}}=110.2 \mathrm{~Hz}\right), 49.9 ;{ }^{31} \mathrm{P} \mathrm{NMR}\left(162 \mathrm{MHz}, \mathrm{CDCl}_{3}\right), \delta(\mathrm{ppm})=19.0$; HRMS (ESI) Calcd for $\mathrm{C}_{27} \mathrm{H}_{21} \mathrm{ClNO}_{3} \mathrm{P}[\mathrm{M}+\mathrm{H}]^{+}=474.1020$, Found $=474.1027$.<smiles>COC(=O)C(=P)C(=O)c1ccnc(Cl)c1</smiles>

\section{Methyl-3-(2-chloropyridin-4-yl)-3-oxo-2-(triphenylphosphoranylidene)propanoat}

-e 37. According to the general procedure A, 2-chloro-4-iodopyridine (119.7 mg, 0.5 mmol), methyl 2-(triphenylphosphoranylidene)acetate (267.4 mg, $0.8 \mathrm{mmol}$ ),

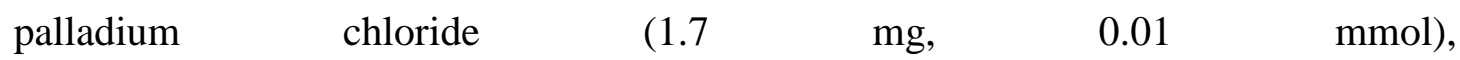
(9,9-dimethyl-9H-xanthene-4,5-diyl)bis(diphenylphosphine) (11.5 mg, $0.02 \mathrm{mmol}$ ), Potassium carbonate $(69.1 \mathrm{mg}, 0.5 \mathrm{mmol}), 1,4$-dioxane $(3 \mathrm{~mL}), \mathrm{CO}(1 \mathrm{~atm}), 100{ }^{\circ} \mathrm{C}$, $24 \mathrm{~h}$, compound 37 was synthesized and purified on silica gel with EtOAc:PE (V:V = $\left.1: 2, \mathrm{R}_{\mathrm{f}}=0.11\right)$ to give a colorless solid $(218 \mathrm{mg}, 92 \%), \mathrm{mp}=162-164{ }^{\circ} \mathrm{C} ;{ }^{1} \mathrm{H} \mathrm{NMR}$ $\left(600 \mathrm{MHz}, \mathrm{CDCl}_{3}\right), \delta(\mathrm{ppm})=8.36(\mathrm{~d}, J=5.0 \mathrm{~Hz}, 1 \mathrm{H}), 7.74-7.71(\mathrm{~m}, 6 \mathrm{H}), 7.60-7.58$ (m, $3 \mathrm{H}), 7.52-7.49(\mathrm{~m}, 6 \mathrm{H}), 7.45(\mathrm{~s}, 1 \mathrm{H}), 7.35(\mathrm{dd}, J=5.0 \mathrm{~Hz}, J=1.0 \mathrm{~Hz}, 1 \mathrm{H}), 3.14$ $(\mathrm{s}, 3 \mathrm{H}) ;{ }^{13} \mathrm{C} \mathrm{NMR}\left(150 \mathrm{MHz}, \mathrm{CDCl}_{3}\right), \delta(\mathrm{ppm})=189.1\left(\mathrm{~d}, J_{\mathrm{P}-\mathrm{C}}=5.7 \mathrm{~Hz}\right), 167.5(\mathrm{~d}, J$ P-C $=13.5 \mathrm{~Hz}), 154.0\left(\mathrm{~d}, J_{\mathrm{P}-\mathrm{C}}=9.7 \mathrm{~Hz}\right), 150.9,149.0,133.4\left(\mathrm{~d}, J_{\mathrm{P}-\mathrm{C}}=9.8 \mathrm{~Hz}\right), 132.3$ $\left(\mathrm{d}, J_{\mathrm{P}-\mathrm{C}}=3.0 \mathrm{~Hz}\right), 128.8\left(\mathrm{~d}, J_{\mathrm{P}-\mathrm{C}}=12.5 \mathrm{~Hz}\right), 125.0\left(\mathrm{~d}, J_{\mathrm{P}-\mathrm{C}}=93.2 \mathrm{~Hz}\right), 122.5,120.8$, $70.9\left(\mathrm{~d}, J_{\mathrm{P}-\mathrm{C}}=110.4 \mathrm{~Hz}\right), 50.0 ;{ }^{31} \mathrm{P}$ NMR $\left(162 \mathrm{MHz}, \mathrm{CDCl}_{3}\right), \delta(\mathrm{ppm})=18.5 ; \mathrm{HRMS}$ (ESI) Calcd for $\mathrm{C}_{27} \mathrm{H}_{21} \mathrm{ClNO}_{3} \mathrm{P}[\mathrm{M}+\mathrm{H}]^{+}=474.1020$, Found $=474.1020$.<smiles>CCOC(=O)C(=P)C(=O)c1ccc(C(=O)OC)cc1</smiles>

Methyl 4-(3-ethoxy-3-oxo-2-(triphenylphosphoranylidene)propanoyl)benzoate 38. According to the general procedure B, methyl 4-iodobenzoate $(183.4 \mathrm{mg}, 0.7 \mathrm{mmol})$, ethyl 2-(triphenyl- $\lambda^{5}$-phosphanylidene)acetate $(174.1 \mathrm{mg}, 0.5 \mathrm{mmol})$, palladium chloride mg, 0.01 mmol), (9,9-dimethyl-9H-xanthene-4,5-diyl)bis(diphenylphosphine) (11.5 mg, $0.02 \mathrm{mmol}$ ), Potassium carbonate $(69.1 \mathrm{mg}, 0.5 \mathrm{mmol}), 1,4$-dioxane $(3 \mathrm{~mL}), \mathrm{CO}(1 \mathrm{~atm}), 100{ }^{\circ} \mathrm{C}$, 
$13 \mathrm{~h}$, compound 38 was synthesized and purified on silica gel with EtOAc:PE (V:V = $\left.1: 2, \mathrm{R}_{\mathrm{f}}=0.18\right)$ to give a colorless solid $(240 \mathrm{mg}, 94 \%), \mathrm{mp}=159-162{ }^{\circ} \mathrm{C} ;{ }^{1} \mathrm{H} \mathrm{NMR}$ $\left(400 \mathrm{MHz}, \mathrm{CDCl}_{3}\right), \delta(\mathrm{ppm})=8.02(\mathrm{~d}, J=8.3 \mathrm{~Hz}, 2 \mathrm{H}), 7.79-7.74(\mathrm{~m}, 6 \mathrm{H}), 7.69(\mathrm{~d}, J$ $=8.2 \mathrm{~Hz}, 2 \mathrm{H}), 7.57-7.54(\mathrm{~m}, 3 \mathrm{H}), 7.51-7.46(\mathrm{~m}, 6 \mathrm{H}), 3.90(\mathrm{~s}, 3 \mathrm{H}), 3.66(\mathrm{q}, J=7.1$ $\mathrm{Hz}, 2 \mathrm{H}), 0.57$ (t, $J=7.1 \mathrm{~Hz}, 3 \mathrm{H}) ;{ }^{13} \mathrm{C} \mathrm{NMR}\left(100 \mathrm{MHz}, \mathrm{CDCl}_{3}\right), \delta(\mathrm{ppm})=192.5(\mathrm{~d}$, $\left.J_{\text {P-C }}=5.5 \mathrm{~Hz}\right), 167.5\left(\mathrm{~d}, J_{\mathrm{P}-\mathrm{C}}=14.1 \mathrm{~Hz}\right), 167.1,148.0\left(\mathrm{~d}, J_{\mathrm{P}-\mathrm{C}}=9.1 \mathrm{~Hz}\right), 133.4(\mathrm{~d}, J$ P-C $=9.7 \mathrm{~Hz}), 132.0\left(\mathrm{~d}, J_{\text {P-C }}=2.8 \mathrm{~Hz}\right), 130.3,128.8,128.7\left(\mathrm{~d}, J_{\text {P-C }}=12.5 \mathrm{~Hz}\right), 127.8$, $125.8\left(\mathrm{~d}, J_{\mathrm{P}-\mathrm{C}}=92.7 \mathrm{~Hz}\right), 69.9\left(\mathrm{~d}, J_{\mathrm{P}-\mathrm{C}}=110.1 \mathrm{~Hz}\right), 58.7,52.0,13.6 ;{ }^{31} \mathrm{P} \mathrm{NMR}(162$ $\left.\mathrm{MHz}, \mathrm{CDCl}_{3}\right), \delta(\mathrm{ppm})=18.5$; HRMS (ESI) Calcd for $\mathrm{C}_{31} \mathrm{H}_{27} \mathrm{O}_{5} \mathrm{P}[\mathrm{M}+\mathrm{H}]^{+}=$ 511.1669 , Found $=511.1667$.<smiles>COC(=O)c1ccc(C(=O)C(=P)C(=P)C(=O)OCc2ccccc2)cc1</smiles>

Methyl-4-(3-(benzyloxy)-3-oxo-2-(triphenylphosphoranylidene)propanoyl)benzoate 39. According to the general procedure $\mathrm{B}$, methyl 4-iodobenzoate $(183.4 \mathrm{mg}, 0.7$ mmol), benzyl 2-(triphenylphosphoranylidene)acetate $(205.2 \mathrm{mg}, 0.5 \mathrm{mmol})$, palladium chloride $\quad(1.7 \quad \mathrm{mg}, 0.01 \quad \mathrm{mmol})$, (9,9-dimethyl-9H-xanthene-4,5-diyl)bis(diphenylphosphine) (11.5 mg, $0.02 \mathrm{mmol}$ ), Potassium carbonate (69.1 mg, $0.5 \mathrm{mmol}), 1,4$-dioxane $(3 \mathrm{~mL}), \mathrm{CO}(1 \mathrm{~atm}), 100{ }^{\circ} \mathrm{C}$, $13 \mathrm{~h}$, compound 39 was synthesized and purified on silica gel with EtOAc:PE (V:V = $\left.1: 2, \mathrm{R}_{\mathrm{f}}=0.16\right)$ to give a colorless solid $(249 \mathrm{mg}, 87 \%), \mathrm{mp}=145-148{ }^{\circ} \mathrm{C} ;{ }^{1} \mathrm{H} \mathrm{NMR}$ $\left(400 \mathrm{MHz}, \mathrm{CDCl}_{3}\right), \delta(\mathrm{ppm})=7.98(\mathrm{~d}, J=8.4 \mathrm{~Hz}, 2 \mathrm{H}), 7.76-7.69(\mathrm{~m}, 8 \mathrm{H}), 7.56-7.51$ (m, $3 \mathrm{H}), 7.47-7.42(\mathrm{~m}, 6 \mathrm{H}), 7.17-7.07(\mathrm{~m}, 3 \mathrm{H}), 6.68(\mathrm{~d}, J=7.2 \mathrm{~Hz}, 2 \mathrm{H}), 4.69$ (s, 2 $\mathrm{H}), 3.92(\mathrm{~s}, 3 \mathrm{H}) ;{ }^{13} \mathrm{C} \mathrm{NMR}\left(100 \mathrm{MHz}, \mathrm{CDCl}_{3}\right), \delta(\mathrm{ppm})=192.6\left(\mathrm{~d}, J_{\mathrm{P}-\mathrm{C}}=5.6 \mathrm{~Hz}\right)$, $167.2\left(\mathrm{~d}, J_{\text {P-C }}=14.4 \mathrm{~Hz}\right), 167.0,147.8\left(\mathrm{~d}, J_{\text {P-C }}=9.3 \mathrm{~Hz}\right), 136.3,133.3\left(\mathrm{~d}, J_{\text {P-C }}=9.8\right.$ $\mathrm{Hz}), 132.0\left(\mathrm{~d}, J_{\text {P-C }}=2.9 \mathrm{~Hz}\right), 130.5,128.9,128.7$ (d, $\left.J_{\text {P-C }}=12.5 \mathrm{~Hz}\right), 128.0,127.9$, 127.4, $125.6\left(\mathrm{~d}, J_{\mathrm{P}-\mathrm{C}}=92.7 \mathrm{~Hz}\right), 69.6\left(\mathrm{~d}, J_{\mathrm{P}-\mathrm{C}}=110.5 \mathrm{~Hz}\right), 65.0,52.0 ;{ }^{31} \mathrm{P}$ NMR $(162$ $\left.\mathrm{MHz}, \mathrm{CDCl}_{3}\right), \delta(\mathrm{ppm})=18.8$; HRMS (ESI) Calcd for $\mathrm{C}_{36} \mathrm{H}_{29} \mathrm{O}_{5} \mathrm{P}[\mathrm{M}+\mathrm{H}]^{+}=$ 573.1825 , Found $=573.1825$. 
<smiles>COC(=O)c1ccc(C(=O)C(=P)C(P)C#N)cc1</smiles>

Methyl 4-(2-cyano-2-(triphenylphosphoranylidene)acetyl)benzoate 40. According to the general procedure $\mathrm{B}$, methyl 4-iodobenzoate $(183.4 \mathrm{mg}, 0.7 \mathrm{mmol})$, 2-(triphenylphosphoranylidene)acetonitrile (150.6 mg, $0.5 \mathrm{mmol})$, palladium chloride (1.7 mg, $0.01 \mathrm{mmol})$, (9,9-dimethyl-9H-xanthene-4,5-diyl)bis(diphenylphosphine) (11.5 mg, $0.02 \mathrm{mmol})$, Potassium carbonate (69.1 mg, $0.5 \mathrm{mmol})$, 1,4-dioxane (3 mL), $\mathrm{CO}(1 \mathrm{~atm}), 100{ }^{\circ} \mathrm{C}, 13 \mathrm{~h}$, compound 40 was synthesized and purified on silica gel with EtOAc:PE $\left(\mathrm{V}: \mathrm{V}=1: 2, \mathrm{R}_{\mathrm{f}}=0.11\right)$ to give a pale yellow solid $(160 \mathrm{mg}, 69 \%), \mathrm{mp}$ $=186-189{ }^{\circ} \mathrm{C} ;{ }^{1} \mathrm{H} \mathrm{NMR}\left(400 \mathrm{MHz}, \mathrm{CDCl}_{3}\right), \delta(\mathrm{ppm})=8.07(\mathrm{~d}, J=8.5 \mathrm{~Hz}, 2 \mathrm{H}), 8.02$ $(\mathrm{d}, J=8.5 \mathrm{~Hz}, 2 \mathrm{H}), 7.72-7.64(\mathrm{~m}, 9 \mathrm{H}), 7.57-7.52(\mathrm{~m}, 6 \mathrm{H}), 3.92(\mathrm{~s}, 3 \mathrm{H}) ;{ }^{13} \mathrm{C} \mathrm{NMR}$ $\left(100 \mathrm{MHz}, \mathrm{CDCl}_{3}\right), \delta(\mathrm{ppm})=189.3\left(\mathrm{~d}, J_{\text {P-C }}=4.2 \mathrm{~Hz}\right), 166.7,143.1\left(\mathrm{~d}, J_{\text {P-C }}=8.7\right.$ $\mathrm{Hz}), 133.7\left(\mathrm{~d}, J_{\mathrm{P}-\mathrm{C}}=10.2 \mathrm{~Hz}\right), 133.2\left(\mathrm{~d}, J_{\mathrm{P}-\mathrm{C}}=2.9 \mathrm{~Hz}\right), 131.8,129.4,129.2\left(\mathrm{~d}, J_{\mathrm{P}-\mathrm{C}}\right.$ $=12.9 \mathrm{~Hz}), 128.0,123.0\left(\mathrm{~d}, J_{\mathrm{P}-\mathrm{C}}=93.3 \mathrm{~Hz}\right), 122.2\left(\mathrm{~d}, J_{\mathrm{P}-\mathrm{C}}=14.9 \mathrm{~Hz}\right), 52.2,49.7(\mathrm{~d}$, $\left.J_{\mathrm{P}-\mathrm{C}}=125.0 \mathrm{~Hz}\right) ;{ }^{31} \mathrm{P} \mathrm{NMR}\left(162 \mathrm{MHz}, \mathrm{CDCl}_{3}\right), \delta(\mathrm{ppm})=22.0$; HRMS (ESI) Calcd for $\mathrm{C}_{29} \mathrm{H}_{22} \mathrm{NO}_{3} \mathrm{P}[\mathrm{M}+\mathrm{H}]^{+}=464.1410$, Found $=464.1412$.<smiles>COC(=O)c1ccc(C(=O)C(=P)C(=O)c2ccccc2)cc1</smiles>

Methyl 4-(3-oxo-3-phenyl-2-(triphenylphosphoranylidene)propanoyl)benzoate 41. According to the general procedure B, methyl 4-iodobenzoate (183.4 mg, $0.7 \mathrm{mmol})$, 1-phenyl-2-(triphenylphosphoranylidene)ethanone (190.0 mg, $0.5 \mathrm{mmol}$ ), palladium chloride (1.7 mg, 0.01 mmol), (9,9-dimethyl-9H-xanthene-4,5-diyl)bis(diphenylphosphine) (11.5 mg, $0.02 \mathrm{mmol}$ ), Potassium carbonate (69.1 mg, $0.5 \mathrm{mmol}), 1,4$-dioxane $(3 \mathrm{~mL}), \mathrm{CO}(1 \mathrm{~atm}), 100{ }^{\circ} \mathrm{C}$, $13 \mathrm{~h}$, compound 41 was synthesized and purified on silica gel with EtOAc:PE (V:V = $\left.1: 2, \mathrm{R}_{\mathrm{f}}=0.13\right)$ to give a yellow solid $(217 \mathrm{mg}, 80 \%), \mathrm{mp}=169-171{ }^{\circ} \mathrm{C} ;{ }^{1} \mathrm{H} \mathrm{NMR}$ $\left(400 \mathrm{MHz}, \mathrm{CDCl}_{3}\right), \delta(\mathrm{ppm})=7.79-7.73(\mathrm{~m}, 6 \mathrm{H}), 7.66(\mathrm{~d}, J=8.4 \mathrm{~Hz}, 2 \mathrm{H}), 7.58-7.55$ 
(m, $3 \mathrm{H})$, 7.51-7.44 (m, $10 \mathrm{H}), 7.07-6.98$ (m, $3 \mathrm{H}), 3.83$ (s, $3 \mathrm{H}) ;{ }^{13} \mathrm{C}$ NMR (100 MHz, $\left.\mathrm{CDCl}_{3}\right), \delta(\mathrm{ppm})=193.2\left(\mathrm{~d}, J_{\mathrm{P}-\mathrm{C}}=6.9 \mathrm{~Hz}\right), 191.7\left(\mathrm{~d}, J_{\mathrm{P}-\mathrm{C}}=6.4 \mathrm{~Hz}\right), 166.7,147.0(\mathrm{~d}$, $\left.J_{\text {P-C }}=8.9 \mathrm{~Hz}\right), 142.6\left(\mathrm{~d}, J_{\text {P-C }}=8.7 \mathrm{~Hz}\right), 133.5\left(\mathrm{~d}, J_{\text {P-C }}=10.1 \mathrm{~Hz}\right), 132.1\left(\mathrm{~d}, J_{\text {P-C }}=\right.$ $2.9 \mathrm{~Hz}), 130.3,130.2,129.1,128.8\left(\mathrm{~d}, J_{\text {P-C }}=12.6 \mathrm{~Hz}\right), 128.6,127.5,125.9\left(\mathrm{~d}, J_{\text {P-C }}=\right.$ $92.0 \mathrm{~Hz}), 83.8\left(\mathrm{~d}, J_{\mathrm{P}-\mathrm{C}}=101.9 \mathrm{~Hz}\right), 52.0 ;{ }^{31} \mathrm{P} \mathrm{NMR}\left(162 \mathrm{MHz}, \mathrm{CDCl}_{3}\right), \delta(\mathrm{ppm})=$ 18.5; HRMS (ESI) Calcd for $\mathrm{C}_{35} \mathrm{H}_{27} \mathrm{O}_{4} \mathrm{P}[\mathrm{M}+\mathrm{H}]^{+}=543.1720$, Found $=543.1716$.

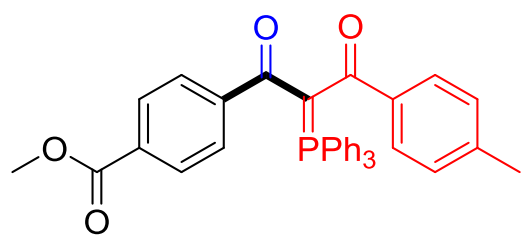

Methyl 4-(3-oxo-3-(p-tolyl)-2-(triphenylphosphoranylidene)propanoyl)benzoate

42. According to the general procedure $B$, methyl 4-iodobenzoate $(183.4 \mathrm{mg}, 0.7$ mmol), 1-(p-tolyl)-2-(triphenylphosphoranylidene)ethanone (197.2 mg, $0.5 \mathrm{mmol}$ ),

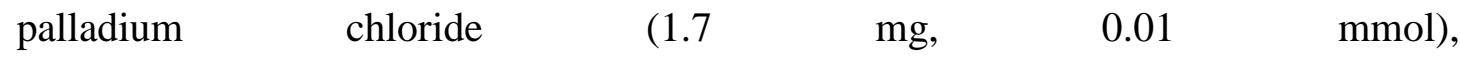
(9,9-dimethyl-9H-xanthene-4,5-diyl)bis(diphenylphosphine) (11.5 mg, $0.02 \mathrm{mmol}$ ), Potassium carbonate (69.1 mg, $0.5 \mathrm{mmol}), 1$,4-dioxane $(3 \mathrm{~mL}), \mathrm{CO}(1 \mathrm{~atm}), 100{ }^{\circ} \mathrm{C}$, $13 \mathrm{~h}$, compound 42 was synthesized and purified on silica gel with EtOAc:PE (V:V = $\left.1: 2, \mathrm{R}_{\mathrm{f}}=0.10\right)$ to give a pale yellow solid $(167 \mathrm{mg}, 60 \%), \mathrm{mp}=150-153{ }^{\circ} \mathrm{C} ;{ }^{1} \mathrm{H}$ NMR (400 MHz, $\left.\mathrm{CDCl}_{3}\right), \delta(\mathrm{ppm})=7.77-7.72(\mathrm{~m}, 6 \mathrm{H}), 7.67(\mathrm{~d}, J=7.4 \mathrm{~Hz}, 2 \mathrm{H})$, 7.56-7.39 (m, $13 \mathrm{H}), 6.80(\mathrm{~d}, J=7.2 \mathrm{~Hz}, 2 \mathrm{H}), 3.84(\mathrm{~s}, 3 \mathrm{H}), 2.14(\mathrm{~s}, 3 \mathrm{H}) ;{ }^{13} \mathrm{C} \mathrm{NMR}$ $\left(150 \mathrm{MHz}, \mathrm{CDCl}_{3}\right), \delta(\mathrm{ppm})=193.0\left(\mathrm{~d}, J_{\mathrm{P}-\mathrm{C}}=7.0 \mathrm{~Hz}\right), 191.3\left(\mathrm{~d}, J_{\mathrm{P}-\mathrm{C}}=6.5 \mathrm{~Hz}\right)$, $166.8,147.0\left(\mathrm{~d}, J_{\text {P-C }}=9.0 \mathrm{~Hz}\right), 140.5,139.8\left(\mathrm{~d}, J_{\text {P-C }}=8.6 \mathrm{~Hz}\right), 133.6\left(\mathrm{~d}, J_{\text {P-C }}=10.0\right.$ $\mathrm{Hz}), 132.0\left(\mathrm{~d}, J_{\text {P-C }}=3.0 \mathrm{~Hz}\right), 130.1,129.3,128.8\left(\mathrm{~d}, J_{\text {P-C }}=12.8 \mathrm{~Hz}\right), 128.5,128.2$, $126.0\left(\mathrm{~d}, J_{\mathrm{P}-\mathrm{C}}=92.1 \mathrm{~Hz}\right), 83.5\left(\mathrm{~d}, J_{\mathrm{P}-\mathrm{C}}=101.9 \mathrm{~Hz}\right), 52.0,21.3 ;{ }^{31} \mathrm{P}$ NMR $(162 \mathrm{MHz}$, $\left.\mathrm{CDCl}_{3}\right), \delta(\mathrm{ppm})=18.5$; HRMS (ESI) Calcd for $\mathrm{C}_{36} \mathrm{H}_{29} \mathrm{O}_{4} \mathrm{P}[\mathrm{M}+\mathrm{H}]^{+}=557.1876$, Found $=557.1874$.<smiles>COC(=O)c1ccc(C(=O)C(=P)C(=O)c2ccc(OC)cc2)cc1</smiles>

Methyl-4-(3-(4-methoxyphenyl)-3-oxo-2-(triphenylphosphoranylidene)propanoyl 
)benzoate 43. According to the general procedure B, methyl 4-iodobenzoate (183.4 mg, $0.7 \mathrm{mmol})$, 1-(4-methoxyphenyl)-2-(triphenylphosphoranylidene)ethanone (205.2 $\mathrm{mg}, \quad 0.5 \mathrm{mmol})$, palladium chloride $(1.7 \mathrm{mg}, 0.01 \mathrm{mmol})$, (9,9-dimethyl-9H-xanthene-4,5-diyl)bis(diphenylphosphine) (11.5 mg, $0.02 \mathrm{mmol}$ ), Potassium carbonate (69.1 mg, $0.5 \mathrm{mmol}), 1$,4-dioxane $(3 \mathrm{~mL}), \mathrm{CO}(1 \mathrm{~atm}), 100{ }^{\circ} \mathrm{C}$, $24 \mathrm{~h}$, compound $\mathbf{4 3}$ was synthesized and purified on silica gel with EtOAc:PE (V:V = $\left.1: 2, \mathrm{R}_{\mathrm{f}}=0.10\right)$ to give a pale yellow solid $(238 \mathrm{mg}, 83 \%), \mathrm{mp}=159-161{ }^{\circ} \mathrm{C} ;{ }^{1} \mathrm{H}$ NMR (400 MHz, $\left.\mathrm{CDCl}_{3}\right), \delta(\mathrm{ppm})=7.77-7.72(\mathrm{~m}, 6 \mathrm{H}), 7.68(\mathrm{~d}, J=8.2 \mathrm{~Hz}, 2 \mathrm{H})$, 7.57-7.44 (m, $13 \mathrm{H}), 6.53(\mathrm{~d}, J=8.2 \mathrm{~Hz}, 2 \mathrm{H}), 3.83(\mathrm{~s}, 3 \mathrm{H}), 3.67(\mathrm{~s}, 3 \mathrm{H}) ;{ }^{13} \mathrm{C} \mathrm{NMR}$ $\left(100 \mathrm{MHz}, \mathrm{CDCl}_{3}\right), \delta(\mathrm{ppm})=192.2\left(J_{\mathrm{P}-\mathrm{C}}=7.6 \mathrm{~Hz}\right), 190.9\left(J_{\mathrm{P}-\mathrm{C}}=6.0 \mathrm{~Hz}\right), 166.7$, 161.3, 147.1 ( $\left.J_{\text {P-C }}=9.2 \mathrm{~Hz}\right), 135.4\left(J_{\text {P-C }}=8.5 \mathrm{~Hz}\right), 133.5\left(J_{\text {P-C }}=10.2 \mathrm{~Hz}\right), 132.0(J$ P-C $=2.9 \mathrm{~Hz}), 131.3,130.1,128.7\left(J_{\text {P-C }}=12.6 \mathrm{~Hz}\right), 128.7,128.4,126.0\left(J_{\text {P-C }}=92.1\right.$ $\mathrm{Hz}), 112.8,83.1\left(J_{\mathrm{P}-\mathrm{C}}=101.7 \mathrm{~Hz}\right), 55.2,52.0 ;{ }^{31} \mathrm{P} \mathrm{NMR}\left(162 \mathrm{MHz}, \mathrm{CDCl}_{3}\right), \delta(\mathrm{ppm})$ $=18.6$; HRMS (ESI) Calcd for $\mathrm{C}_{36} \mathrm{H}_{29} \mathrm{O}_{5} \mathrm{P}[\mathrm{M}+\mathrm{H}]^{+}=573.1825$, Found $=573.1823$.

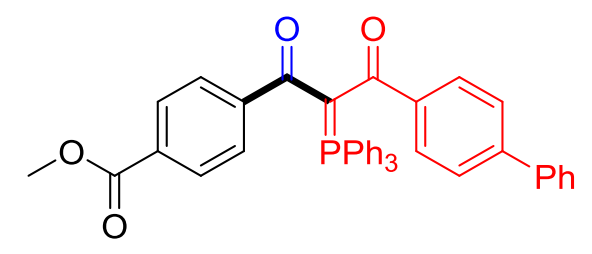

Methyl-4-(3-([1,1'-biphenyl]-4-yl)-3-oxo-2-(triphenylphosphoranylidene)propano

yl)benzoate 44. According to the general procedure B, methyl 4-iodobenzoate (183.4 mg, $0.7 \mathrm{mmol}), \quad$ 1-([1,1'-biphenyl]-4-yl)-2-(triphenylphosphoranylidene)ethanone (228.2 mg, $0.5 \mathrm{mmol})$, palladium chloride $(1.7 \mathrm{mg}, 0.01 \mathrm{mmol})$, (9,9-dimethyl-9H-xanthene-4,5-diyl)bis(diphenylphosphine) (11.5 mg, $0.02 \mathrm{mmol}$ ), Potassium carbonate (69.1 mg, $0.5 \mathrm{mmol}), 1,4$-dioxane $(3 \mathrm{~mL}), \mathrm{CO}(1 \mathrm{~atm}), 100{ }^{\circ} \mathrm{C}$, $24 \mathrm{~h}$, compound 44 was synthesized and purified on silica gel with EtOAc:PE (V:V = $\left.1: 2, \mathrm{R}_{\mathrm{f}}=0.11\right)$ to give a pale yellow solid $(238 \mathrm{mg}, 77 \%), \mathrm{mp}=165-168{ }^{\circ} \mathrm{C} ;{ }^{1} \mathrm{H}$ $\operatorname{NMR}\left(600 \mathrm{MHz}, \mathrm{CDCl}_{3}\right), \delta(\mathrm{ppm})=7.80-7.76(\mathrm{~m}, 6 \mathrm{H}), 7.67(\mathrm{~d}, J=7.9 \mathrm{~Hz}, 2 \mathrm{H})$, 7.59-7.56 (m, 3 H), 7.54-7.47 (m, 10 H), 7.38-7.36 (m, 4 H), 7.32-7.30 (m, 1 H), 7.22 $(\mathrm{d}, J=7.9 \mathrm{~Hz}, 2 \mathrm{H}), 3.78(\mathrm{~s}, 3 \mathrm{H}) ;{ }^{13} \mathrm{C} \mathrm{NMR}\left(100 \mathrm{MHz}, \mathrm{CDCl}_{3}\right), \delta(\mathrm{ppm})=192.7(\mathrm{~d}$, $\left.J_{\text {P-C }}=6.8 \mathrm{~Hz}\right), 191.6\left(\mathrm{~d}, J_{\text {P-C }}=6.1 \mathrm{~Hz}\right), 166.6,146.9\left(\mathrm{~d}, J_{\text {P-C }}=8.8 \mathrm{~Hz}\right), 142.8,141.3$ 
$\left(\mathrm{d}, J_{\mathrm{P}-\mathrm{C}}=8.6 \mathrm{~Hz}\right), 140.5,133.6\left(\mathrm{~d}, J_{\mathrm{P}-\mathrm{C}}=10.0 \mathrm{~Hz}\right), 132.1\left(\mathrm{~d}, J_{\mathrm{P}-\mathrm{C}}=2.8 \mathrm{~Hz}\right), 130.3$, $129.6,128.8\left(\mathrm{~d}, J_{\text {P-C }}=12.3 \mathrm{~Hz}\right), 128.7,128.6,127.5,127.1,126.3,125.9\left(\mathrm{~d}, J_{\text {P-C }}=\right.$ $92.0 \mathrm{~Hz}), 84.1\left(\mathrm{~d}, J_{\mathrm{P}-\mathrm{C}}=101.2 \mathrm{~Hz}\right), 52.0 ;{ }^{31} \mathrm{P} \mathrm{NMR}\left(162 \mathrm{MHz}, \mathrm{CDCl}_{3}\right), \delta(\mathrm{ppm})=$ 18.5; HRMS (ESI) Calcd for $\mathrm{C}_{41} \mathrm{H}_{31} \mathrm{O}_{4} \mathrm{P}[\mathrm{M}+\mathrm{H}]^{+}=619.2033$, Found $=619.2035$.<smiles>COC(=O)c1ccc(C(=O)C(=P)C(=O)c2ccc(C#N)cc2)cc1</smiles>

Methyl-4-(3-(4-cyanophenyl)-3-oxo-2-(triphenylphosphoranylidene)propanoyl)be nzoate 45. According to the general procedure B, methyl 4-iodobenzoate $(262.0 \mathrm{mg}$, $1.0 \mathrm{mmol}), 4$-(2-(triphenylphosphoranylidene)acetyl)benzonitrile (202.7 $\mathrm{mg}, \quad 0.5$ mmol), palladium chloride $(1.7 \quad \mathrm{mg}, \quad 0.01 \quad \mathrm{mmol})$, (9,9-dimethyl-9H-xanthene-4,5-diyl)bis(diphenylphosphine) (11.5 mg, $0.02 \mathrm{mmol}$ ), Potassium carbonate $(69.1 \mathrm{mg}, 0.5 \mathrm{mmol}), 1,4$-dioxane $(3 \mathrm{~mL}), \mathrm{CO}(1 \mathrm{~atm}), 100{ }^{\circ} \mathrm{C}$, $24 \mathrm{~h}$, compound 45 was synthesized and purified on silica gel with EtOAc:PE (V:V = $\left.1: 2, \mathrm{R}_{\mathrm{f}}=0.10\right)$ to give a yellow solid $(108 \mathrm{mg}, 38 \%), \mathrm{mp}=158-161{ }^{\circ} \mathrm{C} ;{ }^{1} \mathrm{H} \mathrm{NMR}$ $\left(400 \mathrm{MHz}, \mathrm{CDCl}_{3}\right), \delta(\mathrm{ppm})=7.76-7.70(\mathrm{~m}, 8 \mathrm{H}), 7.60-7.46(\mathrm{~m}, 13 \mathrm{H}), 7.29(\mathrm{~d}, J=$ $8.1 \mathrm{~Hz}, 2 \mathrm{H}), 3.86(\mathrm{~s}, 3 \mathrm{H}) ;{ }^{13} \mathrm{C} \mathrm{NMR}\left(100 \mathrm{MHz}, \mathrm{CDCl}_{3}\right), \delta(\mathrm{ppm})=191.9\left(\mathrm{~d}, J_{\mathrm{P}-\mathrm{C}}=\right.$ $6.8 \mathrm{~Hz}), 190.7\left(\mathrm{~d}, J_{\text {P-C }}=6.6 \mathrm{~Hz}\right), 166.3,146.7\left(\mathrm{~d}, J_{\mathrm{P}-\mathrm{C}}=9.0 \mathrm{~Hz}\right), 146.5\left(\mathrm{~d}, J_{\text {P-C }}=8.7\right.$ $\mathrm{Hz}), 133.5\left(\mathrm{~d}, J_{\mathrm{P}-\mathrm{C}}=10.1 \mathrm{~Hz}\right), 132.4\left(\mathrm{~d}, J_{\mathrm{P}-\mathrm{C}}=2.9 \mathrm{~Hz}\right), 131.4,131.2,129.1,128.9(\mathrm{~d}$, $\left.J_{\mathrm{P}-\mathrm{C}}=12.8 \mathrm{~Hz}\right), 128.8,125.2\left(\mathrm{~d}, J_{\mathrm{P}-\mathrm{C}}=92.0 \mathrm{~Hz}\right), 118.4,112.9,84.5\left(\mathrm{~d}, J_{\mathrm{P}-\mathrm{C}}=101.4\right.$ $\mathrm{Hz}), 52.2 ;{ }^{31} \mathrm{P}$ NMR $\left(162 \mathrm{MHz}, \mathrm{CDCl}_{3}\right), \delta(\mathrm{ppm})=18.8$; HRMS (ESI) Calcd for $\mathrm{C}_{36} \mathrm{H}_{26} \mathrm{NO}_{4} \mathrm{P}[\mathrm{M}+\mathrm{H}]^{+}=568.1672$, Found $=568.1668$.<smiles>COC(=O)c1ccc(C(=O)C(=P)C(=O)c2ccc3ccccc3c2)cc1</smiles>

Methyl-4-(3-(naphthalen-2-yl)-3-oxo-2-(triphenylphosphoranylidene)propanoyl)

benzoate 46. According to the general procedure B, methyl 4-iodobenzoate (262.0 mg, $1.0 \mathrm{mmol})$, 1-(naphthalen-2-yl)-2-(triphenylphosphoranylidene)ethanone (215.2 $\mathrm{mg}, \quad 0.5 \mathrm{mmol}), \quad$ palladium chloride $(1.7 \mathrm{mg}, \quad 0.01 \mathrm{mmol})$, 
(9,9-dimethyl-9H-xanthene-4,5-diyl)bis(diphenylphosphine) (11.5 mg, $0.02 \mathrm{mmol}$ ), Potassium carbonate $(69.1 \mathrm{mg}, 0.5 \mathrm{mmol}), 1$,4-dioxane $(3 \mathrm{~mL}), \mathrm{CO}(1 \mathrm{~atm}), 100{ }^{\circ} \mathrm{C}$, $24 \mathrm{~h}$, compound 46 was synthesized and purified on silica gel with EtOAc:PE (V:V = $\left.1: 2, \mathrm{R}_{\mathrm{f}}=0.11\right)$ to give a pale yellow solid $(184 \mathrm{mg}, 62 \%), \mathrm{mp}=77-81{ }^{\circ} \mathrm{C} ;{ }^{1} \mathrm{H}$ NMR $\left(400 \mathrm{MHz}, \mathrm{CDCl}_{3}\right), \delta(\mathrm{ppm})=7.98(\mathrm{~s}, 1 \mathrm{H}), 7.82-7.77(\mathrm{~m}, 6 \mathrm{H}), 7.68-7.46(\mathrm{~m}, 17 \mathrm{H})$, 7.39-7.37 (m, $2 \mathrm{H}), 3.73$ (s, $3 \mathrm{H}) ;{ }^{13} \mathrm{C} \mathrm{NMR}\left(100 \mathrm{MHz}, \mathrm{CDCl}_{3}\right), \delta(\mathrm{ppm})=193.0(\mathrm{~d}, J$ P-C $=7.0 \mathrm{~Hz}), 191.9\left(\mathrm{~d}, J_{\mathrm{P}-\mathrm{C}}=6.6 \mathrm{~Hz}\right), 166.4,147.2\left(\mathrm{~d}, J_{\mathrm{P}-\mathrm{C}}=9.0 \mathrm{~Hz}\right), 139.9\left(\mathrm{~d}, J_{\text {P-C }}\right.$ $=8.7 \mathrm{~Hz}), 134.0,133.6\left(\mathrm{~d}, J_{\text {P-C }}=10.1 \mathrm{~Hz}\right), 132.1\left(\mathrm{~d}, J_{\text {P-C }}=2.9 \mathrm{~Hz}\right), 132.0,130.1$, $129.9,128.8\left(\mathrm{~d}, J_{\text {P-C }}=12.4 \mathrm{~Hz}\right), 128.7,128.6,128.2,127.5\left(\mathrm{~d}, J_{\mathrm{P}-\mathrm{C}}=7.0 \mathrm{~Hz}\right), 126.9$, 126.0, $125.9\left(\mathrm{~d}, J_{\mathrm{P}-\mathrm{C}}=91.9 \mathrm{~Hz}\right), 125.7,83.8\left(\mathrm{~d}, J_{\mathrm{P}-\mathrm{C}}=101.1 \mathrm{~Hz}\right), 51.9 ;{ }^{31} \mathrm{P} \mathrm{NMR}$ $\left(162 \mathrm{MHz}, \mathrm{CDCl}_{3}\right), \delta(\mathrm{ppm})=18.6$; HRMS (ESI) Calcd for $\mathrm{C}_{39} \mathrm{H}_{29} \mathrm{O}_{4} \mathrm{P}[\mathrm{M}+\mathrm{H}]^{+}=$ 593.1876, Found $=593.1877$.

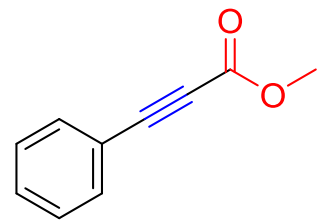

Methyl 3-phenylpropiolate 47. According to the general procedure C, methyl 3-oxo-3-phenyl-2-(triphenylphosphoranylidene)propanoate (438.4 mg, $1 \mathrm{mmol}$ ), $300{ }^{\circ} \mathrm{C}$, 13 minutes, compound 47 was synthesized and purified on silica gel with EtOAc:PE $\left(\mathrm{V}: \mathrm{V}=1: 50, \mathrm{R}_{\mathrm{f}}=0.29\right)$ to give a pale yellow liquid $(125 \mathrm{mg}, 78 \%) .{ }^{1} \mathrm{H}$ NMR (400 MHz, $\left.\mathrm{CDCl}_{3}\right), \delta(\mathrm{ppm})=7.58(\mathrm{~d}, J=7.3 \mathrm{~Hz}, 2 \mathrm{H}), 7.47-7.43(\mathrm{~m}, 1 \mathrm{H})$, 7.39-7.35 (m, $2 \mathrm{H}), 3.84(\mathrm{~s}, 3 \mathrm{H}) ;{ }^{13} \mathrm{C} \mathrm{NMR}\left(100 \mathrm{MHz}, \mathrm{CDCl}_{3}\right), \delta(\mathrm{ppm})=154.5$, 133.0, 130.7, 128.6, 119.5, 86.5, 80.4, 52.8; HRMS (ESI) Calcd for $\mathrm{C}_{10} \mathrm{H}_{8} \mathrm{O}_{2}[\mathrm{M}+$ $\mathrm{Na}]^{+}=183.0417$, Found $=183.0414$

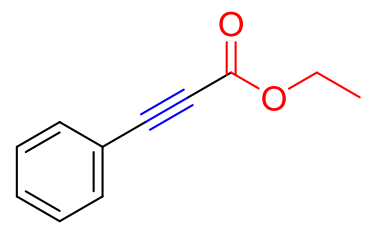

Ethyl 3-phenylpropiolate 48. According to the general procedure C, ethyl 3-oxo-3-phenyl-2-(triphenylphosphoranylidene)propanoate (452.4 $\mathrm{mg}, 1 \mathrm{mmol}$ ), $300{ }^{\circ} \mathrm{C}, 5$ minutes, compound 48 was synthesized and purified on silica gel with 
EtOAc:PE $\left(\mathrm{V}: \mathrm{V}=1: 10, \mathrm{R}_{\mathrm{f}}=0.67\right)$ to give a yellow liquid $(136 \mathrm{mg}, 78 \%) .{ }^{1} \mathrm{H} \mathrm{NMR}$ $\left(400 \mathrm{MHz}, \mathrm{CDCl}_{3}\right), \delta(\mathrm{ppm})=7.59(\mathrm{~d}, J=7.0 \mathrm{~Hz}, 2 \mathrm{H}), 7.47-7.43(\mathrm{~m}, 1 \mathrm{H})$, 7.39-7.36 $(\mathrm{m}, 2 \mathrm{H}), 4.30(\mathrm{q}, J=7.1 \mathrm{~Hz}, 2 \mathrm{H}), 1.36(\mathrm{t}, J=7.2 \mathrm{~Hz}, 3 \mathrm{H}) ;{ }^{13} \mathrm{C}$ NMR $(100 \mathrm{MHz}$, $\left.\mathrm{CDCl}_{3}\right), \delta(\mathrm{ppm})=154.1,133.0,130.6,128.6,119.6,86.1,80.7,62.1,14.1$; HRMS (ESI) Calcd for $\mathrm{C}_{11} \mathrm{H}_{10} \mathrm{O}_{2}[\mathrm{M}+\mathrm{Na}]^{+}=197.0573$, Found $=197.0573$.

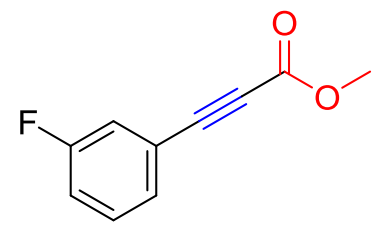

Methyl 3-(3-fluorophenyl)propiolate 49. According to the general procedure C, methyl 3-(3-fluorophenyl)-3-oxo-2-(triphenylphosphoranylidene)propanoate (456.4 $\mathrm{mg}, 1 \mathrm{mmol}), 300{ }^{\circ} \mathrm{C}, 13$ minutes, compound 49 was synthesized and purified on silica gel with EtOAc:PE (V:V $\left.=1: 50, \mathrm{R}_{\mathrm{f}}=0.23\right)$ to give a pale yellow liquid (128 $\mathrm{mg}, 72 \%) .{ }^{1} \mathrm{H}$ NMR $\left(400 \mathrm{MHz}, \mathrm{CDCl}_{3}\right), \delta(\mathrm{ppm})=7.38-7.33(\mathrm{~m}, 2 \mathrm{H}), 7.29-7.26(\mathrm{~m}$, $1 \mathrm{H}), 7.20-7.14(\mathrm{~m}, 1 \mathrm{H}), 3.85(\mathrm{~s}, 3 \mathrm{H}) ;{ }^{13} \mathrm{C} \mathrm{NMR}\left(100 \mathrm{MHz}, \mathrm{CDCl}_{3}\right), \delta(\mathrm{ppm})=162.2$ $\left(\mathrm{d}, J_{\mathrm{F}-\mathrm{C}}=246.5 \mathrm{~Hz}\right), 154.1,130.4\left(\mathrm{~d}, J_{\mathrm{F}-\mathrm{C}}=8.5 \mathrm{~Hz}\right), 128.9\left(\mathrm{~d}, J_{\mathrm{F}-\mathrm{C}}=3.1 \mathrm{~Hz}\right), 121.3$ $\left(\mathrm{d}, J_{\mathrm{F}-\mathrm{C}}=9.2 \mathrm{~Hz}\right), 119.6\left(\mathrm{~d}, J_{\mathrm{F}-\mathrm{C}}=23.1 \mathrm{~Hz}\right), 118.2\left(\mathrm{~d}, J_{\mathrm{F}-\mathrm{C}}=21.1 \mathrm{~Hz}\right), 84.7\left(\mathrm{~d}, J_{\mathrm{F}-\mathrm{C}}\right.$ $=3.5 \mathrm{~Hz}$ ), 80.8, 52.9; HRMS (ESI) Calcd for $\mathrm{C}_{10} \mathrm{H}_{7} \mathrm{FO}_{2}[\mathrm{M}+\mathrm{Na}]^{+}=201.0322$, Found $=201.0323$.

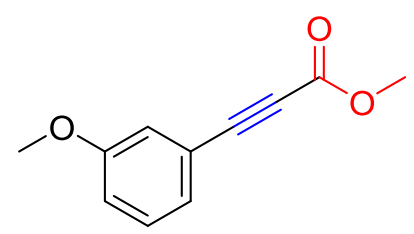

Methyl 3-(3-methoxyphenyl)propiolate 50. According to the general procedure C, methyl 3-(3-methoxyphenyl)-3-oxo-2-(triphenylphosphoranylidene)propanoate (468.4 $\mathrm{mg}, 1 \mathrm{mmol}$ ), $300{ }^{\circ} \mathrm{C}, 8$ minutes, compound $\mathbf{5 0}$ was synthesized and purified on silica gel with EtOAc:PE $\left(\mathrm{V}: \mathrm{V}=1: 10, \mathrm{R}_{\mathrm{f}}=0.52\right)$ to give a pale yellow liquid $(91 \mathrm{mg}, 48 \%)$. ${ }^{1} \mathrm{H}$ NMR $\left(400 \mathrm{MHz}, \mathrm{CDCl}_{3}\right), \delta(\mathrm{ppm})=7.29(\mathrm{~d}, J=7.84 \mathrm{~Hz}, 1 \mathrm{H}), 7.18(\mathrm{~d}, J=7.52$ $\mathrm{Hz}, 1 \mathrm{H}), 7.09$ (s, $1 \mathrm{H}), 7.02-6.99$ (m, $1 \mathrm{H}), 3.84$ (s, $3 \mathrm{H}), 3.81$ (s, $3 \mathrm{H}) ;{ }^{13} \mathrm{C}$ NMR (100 $\left.\mathrm{MHz}, \mathrm{CDCl}_{3}\right), \delta(\mathrm{ppm})=159.3,154.5,129.7,125.5,120.4,117.6,117.4,86.4,80.0$, 55.4, 52.9; HRMS (ESI) Calcd for $\mathrm{C}_{11} \mathrm{H}_{10} \mathrm{O}_{3}[\mathrm{M}+\mathrm{Na}]^{+}=213.0522$, Found $=$ 
213.0521 .

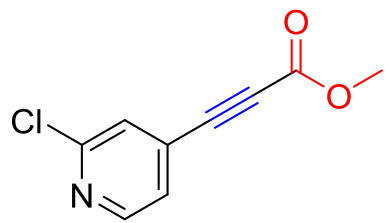

Methyl 3-(2-chloropyridin-4-yl)propiolate 51. According to the general procedure C, methyl 3-(2-chloropyridin-4-yl)-3-oxo-2-(triphenylphosphoranylidene)propanoate (473.8 mg, $1 \mathrm{mmol}$ ), $300{ }^{\circ} \mathrm{C}, 5$ minutes, compound $\mathbf{5 1}$ was synthesized and purified on silica gel with EtOAc:PE $\left(\mathrm{V}: \mathrm{V}=1: 10, \mathrm{R}_{\mathrm{f}}=0.21\right)$ to give a colorless solid $(88 \mathrm{mg}$, $45 \%), \mathrm{mp}=83-85^{\circ} \mathrm{C} ;{ }^{1} \mathrm{H} \mathrm{NMR}\left(400 \mathrm{MHz}, \mathrm{CDCl}_{3}\right), \delta(\mathrm{ppm})=8.45(\mathrm{~d}, J=5.0 \mathrm{~Hz}, 1$ $\mathrm{H}), 7.47(\mathrm{~s}, 1 \mathrm{H}), 7.35(\mathrm{~d}, J=4.9 \mathrm{~Hz}, 1 \mathrm{H}), 3.87(\mathrm{~s}, 3 \mathrm{H}) ;{ }^{13} \mathrm{C}$ NMR $(100 \mathrm{MHz}$, $\left.\mathrm{CDCl}_{3}\right), \delta(\mathrm{ppm})=153.3,152.0,150.1,130.6,126.8,124.7,84.4,80.9,53.3 ;$ HRMS (ESI) Calcd for $\mathrm{C}_{9} \mathrm{H}_{6} \mathrm{ClNO}_{2}[\mathrm{M}+\mathrm{Na}]^{+}=217.9979$, Found $=217.9975$. 
6. Copies of the ${ }^{1} \mathrm{H}$ NMR, ${ }^{13} \mathrm{C}$ NMR and ${ }^{31} \mathrm{P}$ NMR spectra of products.

Methyl 4-(3-methoxy-3-oxo-2-(triphenylphosphoranylidene)propanoyl)benzoate 3: ${ }^{1} \mathrm{H}$ NMR (600 MHz, $\left.\mathrm{CDCl}_{3}\right)$

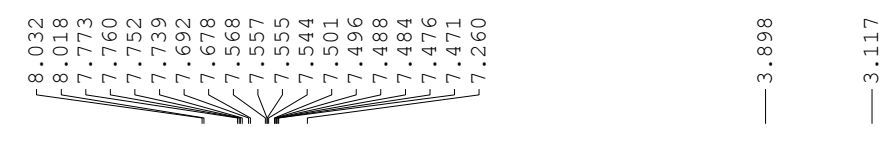<smiles>COC(=O)C(=O)C(=O)c1ccc(C(=O)OC)cc1</smiles>

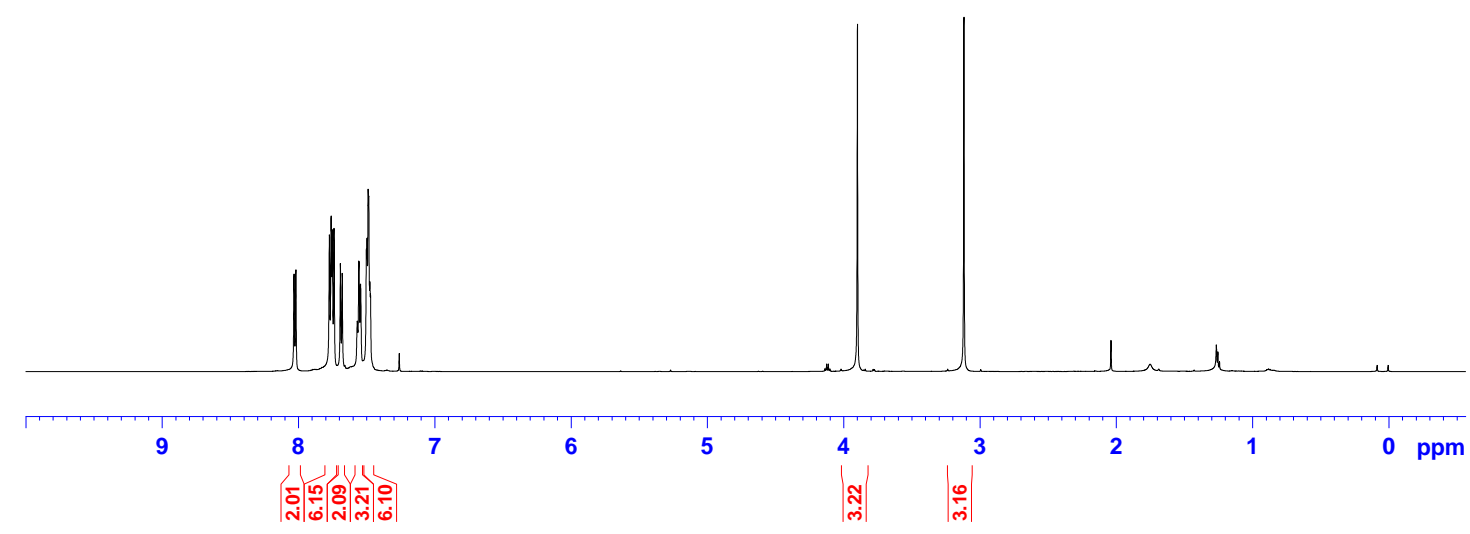

${ }^{13} \mathrm{C}$ NMR (150 MHz, $\left.\mathrm{CDCl}_{3}\right)$
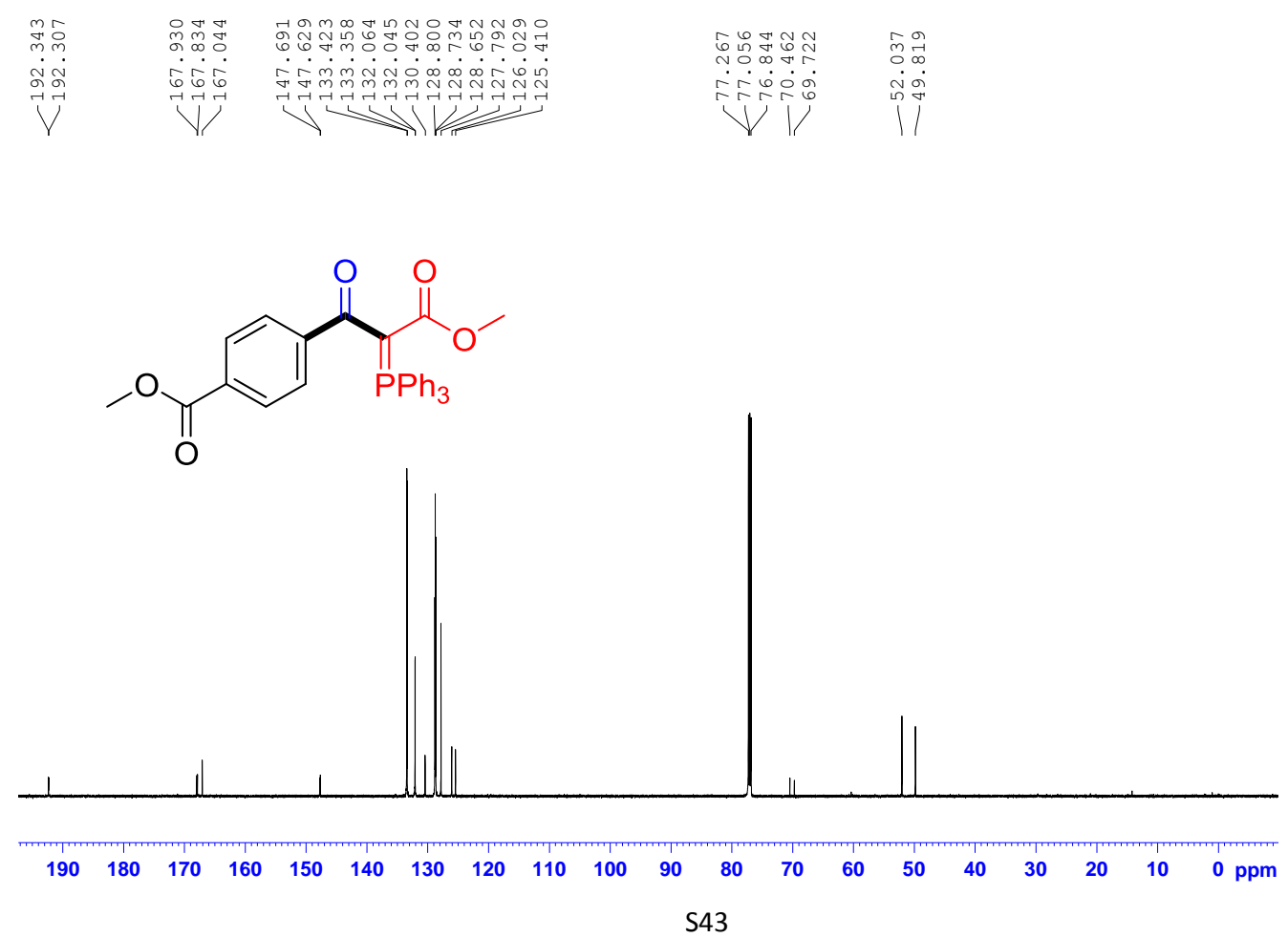
${ }^{31}$ P NMR (162 MHz, $\left.\mathrm{CDCl}_{3}\right)$<smiles>COC(=O)C(=P)C(=O)c1ccc(C(=O)OC)cc1</smiles>

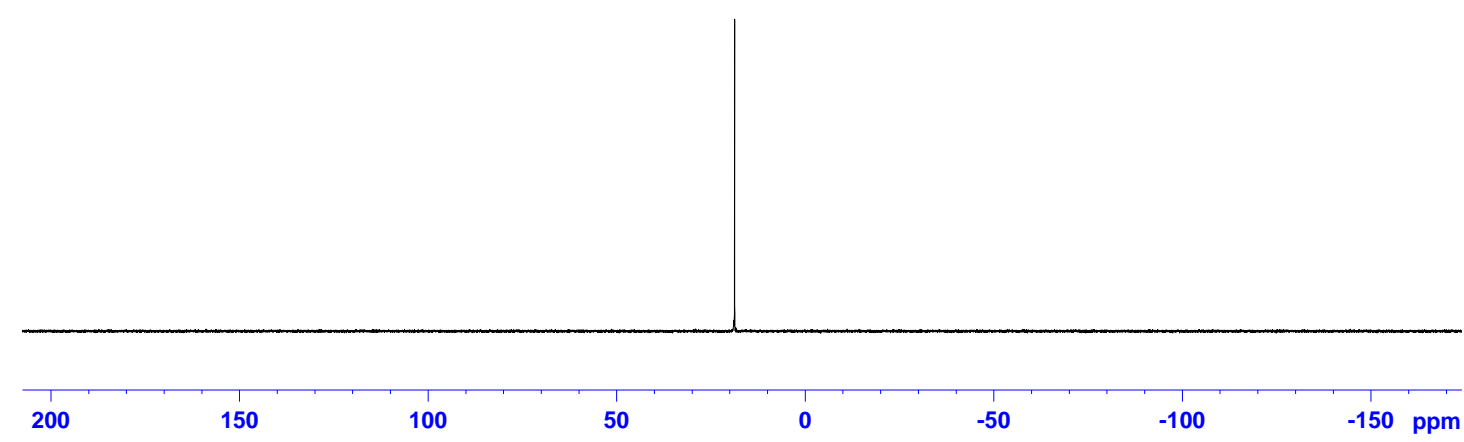

Methyl 3-(4-acetylphenyl)-3-oxo-2-(triphenylphosphoranylidene)propanoate 4:

${ }^{1}$ H NMR (400 MHz, $\left.\mathrm{CDCl}_{3}\right)$

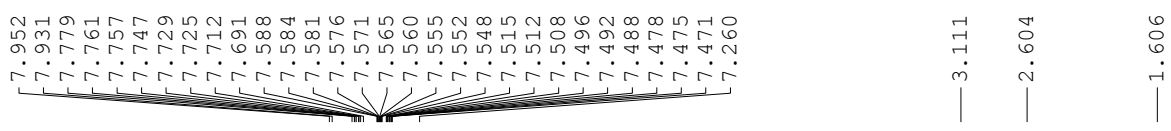

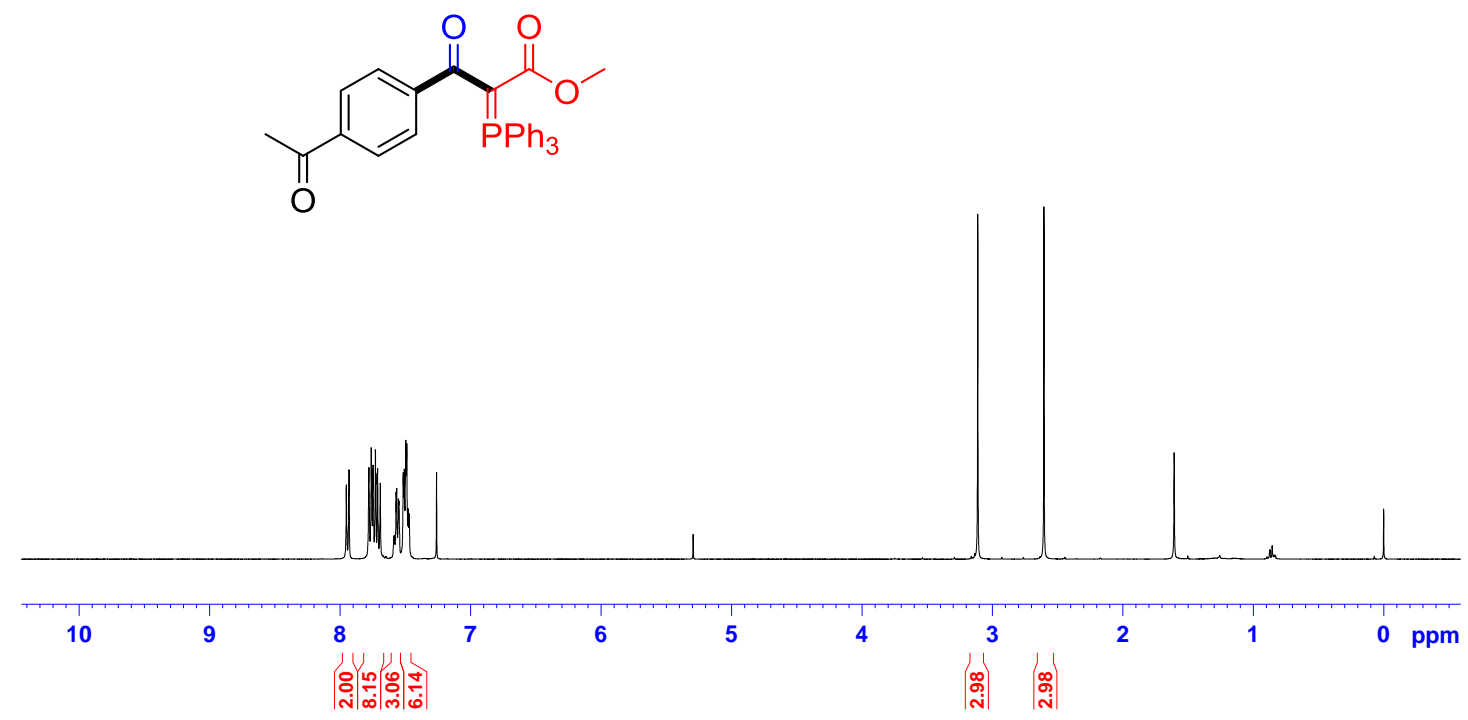


${ }^{13} \mathrm{C}$ NMR (100 MHz, $\left.\mathrm{CDCl}_{3}\right)$

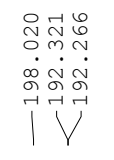
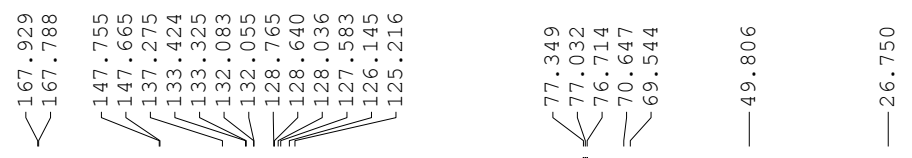<smiles>COC(=O)C(PP)C(=O)c1ccc(C(C)=O)cc1</smiles>

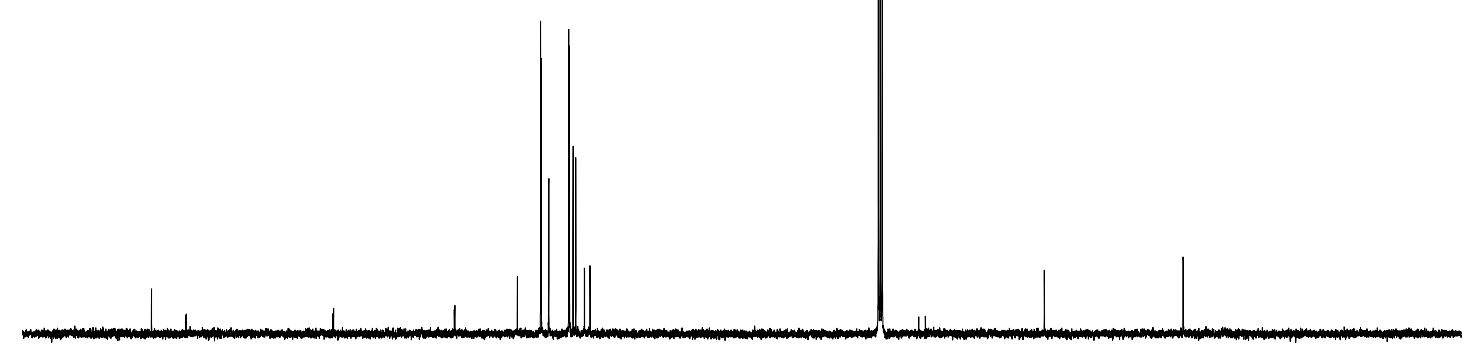

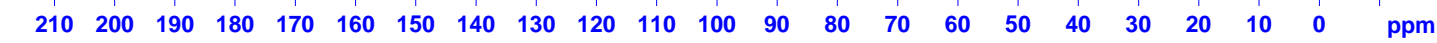
${ }^{31}$ P NMR (162 MHz, $\left.\mathrm{CDCl}_{3}\right)$<smiles>COC(=O)C(C(=O)c1ccc(C(C)=O)cc1)P(P)c1ccccc1</smiles>

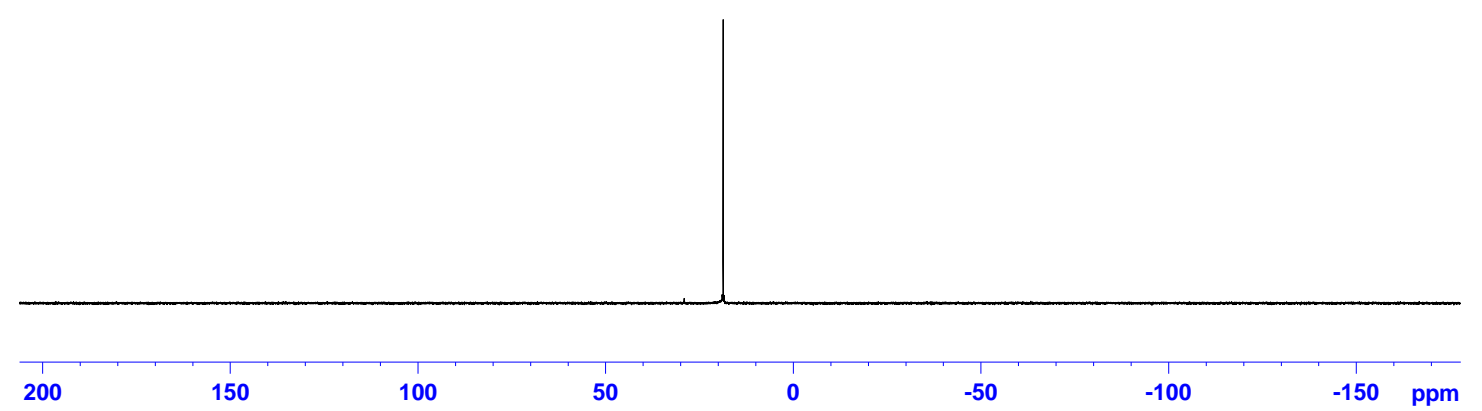


Methyl 3-(4-fluorophenyl)-3-oxo-2-(triphenylphosphoranylidene)propanoate 5:

${ }^{1} \mathrm{H}$ NMR (400 MHz, $\left.\mathrm{CDCl}_{3}\right)$

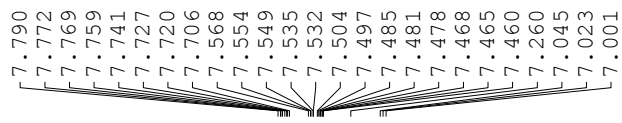<smiles>COC(=O)C(=Pc1ccccc1)C(=O)c1ccc(F)cc1</smiles>

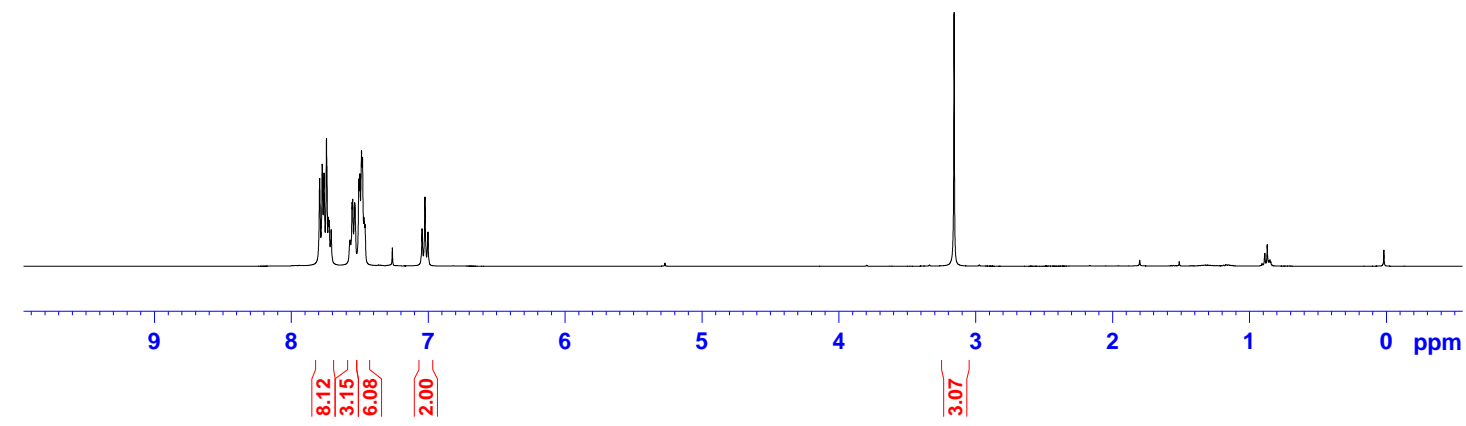

\section{${ }^{13} \mathrm{C}$ NMR (100 MHz, $\left.\mathrm{CDCl}_{3}\right)$}

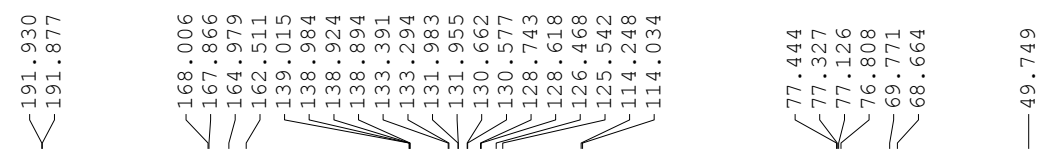<smiles>COC(=O)C(=P)C(=O)c1ccc(F)cc1</smiles>

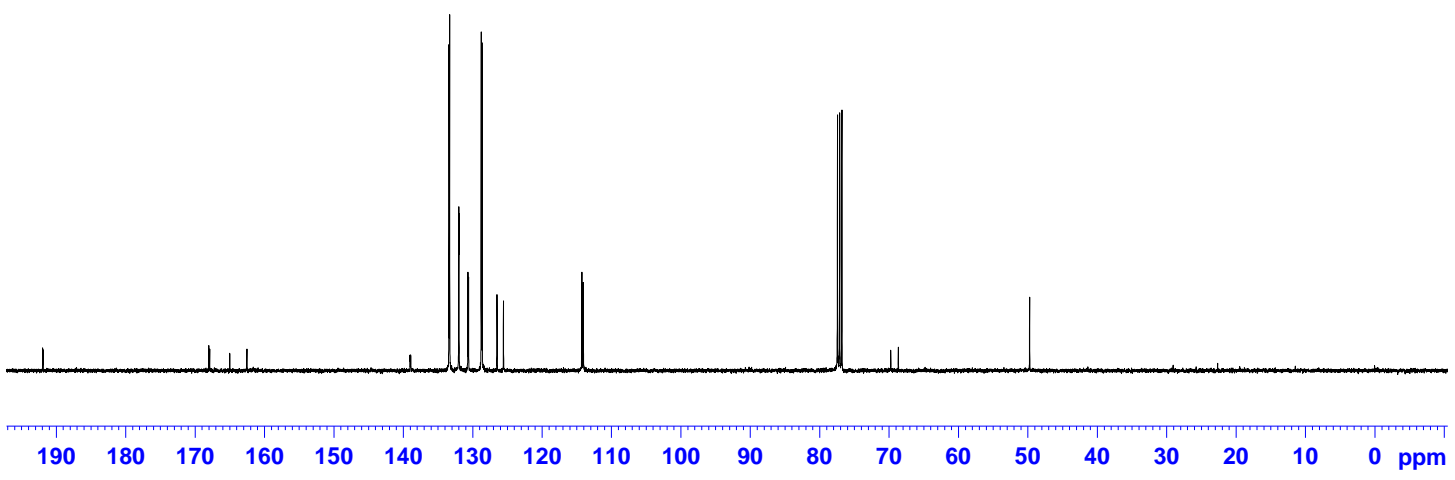


${ }^{31}$ P NMR (162 MHz, $\left.\mathrm{CDCl}_{3}\right)$<smiles>COC(=O)C(=Pc1ccccc1)C(=O)c1ccc(F)cc1</smiles>

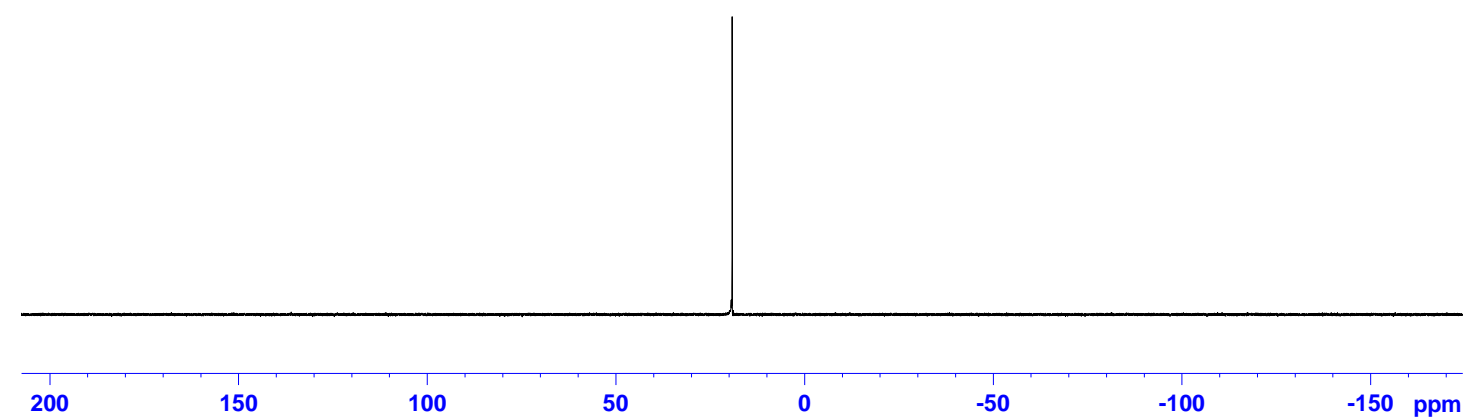

Methyl 3-(4-chlorophenyl)-3-oxo-2-(triphenylphosphoranylidene)propanoate 6:

${ }^{1} \mathrm{H}$ NMR (400 MHz, $\left.\mathrm{CDCl}_{3}\right)$

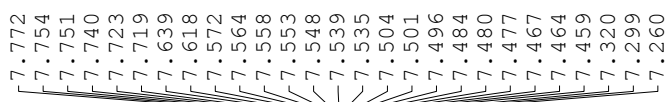<smiles>COC(=O)C(=Pc1ccccc1)C(=O)c1ccc(Cl)cc1</smiles>

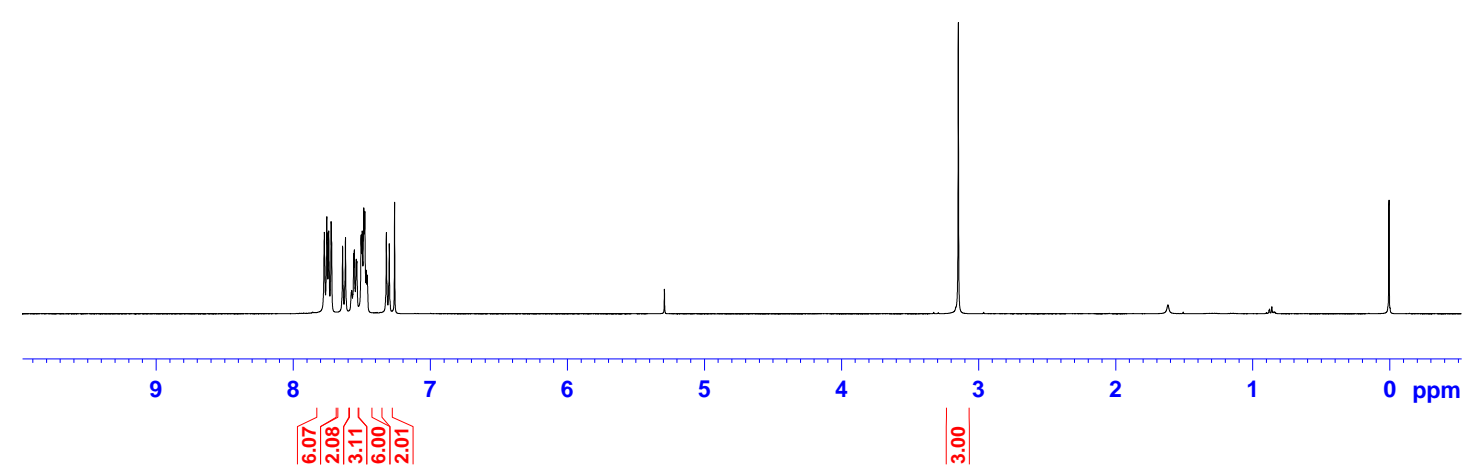


${ }^{13} \mathrm{C}$ NMR (100 MHz, $\left.\mathrm{CDCl}_{3}\right)$

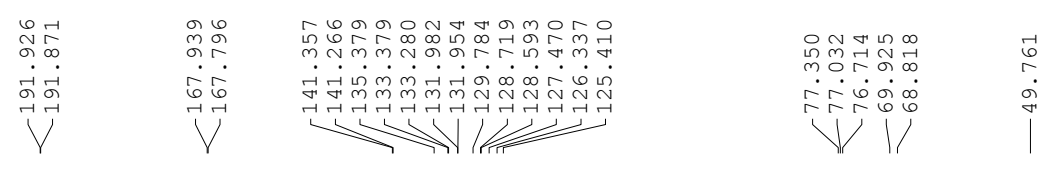

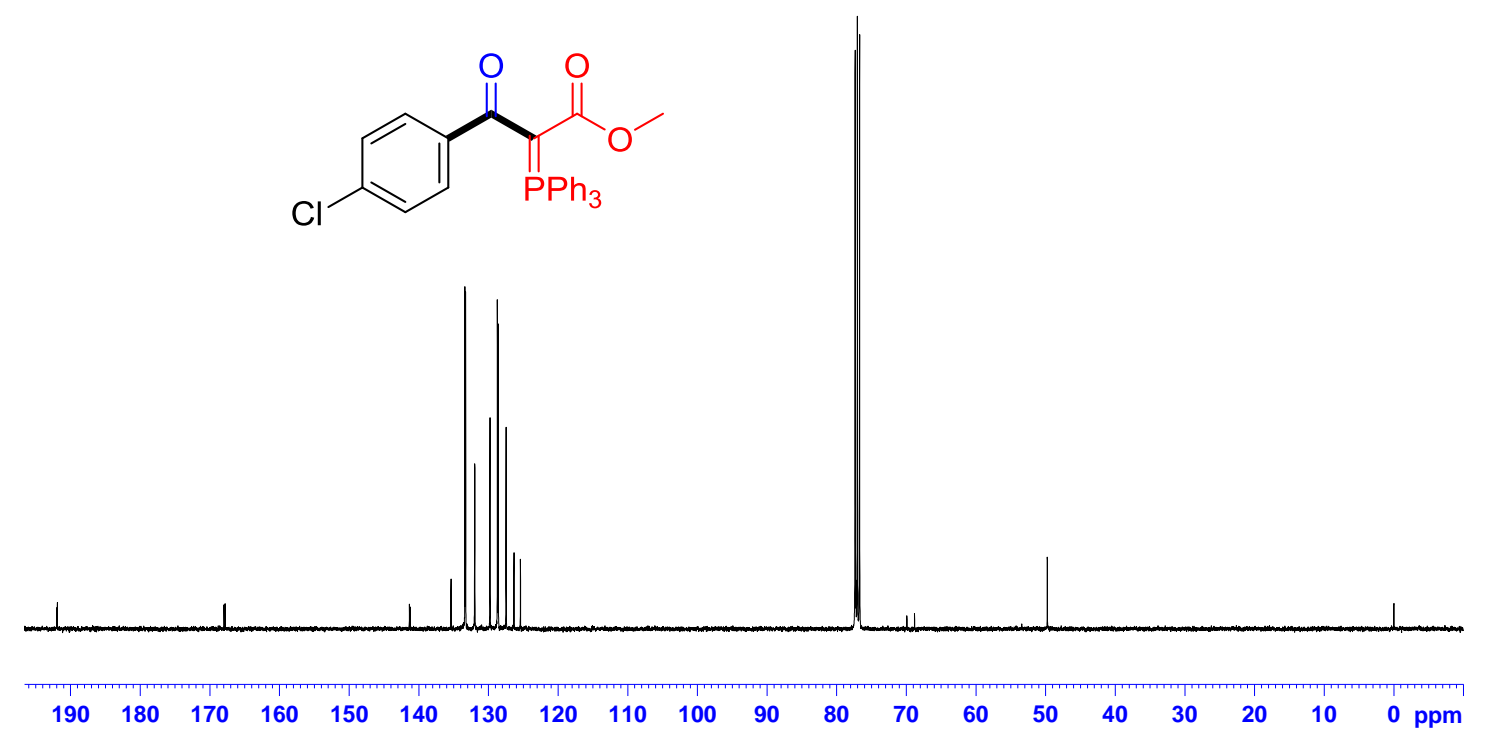

${ }^{31}$ P NMR (162 MHz, $\mathrm{CDCl}_{3}$ )<smiles>COC(=O)C(=Pc1ccccc1)C(=O)c1ccc(Cl)cc1</smiles>

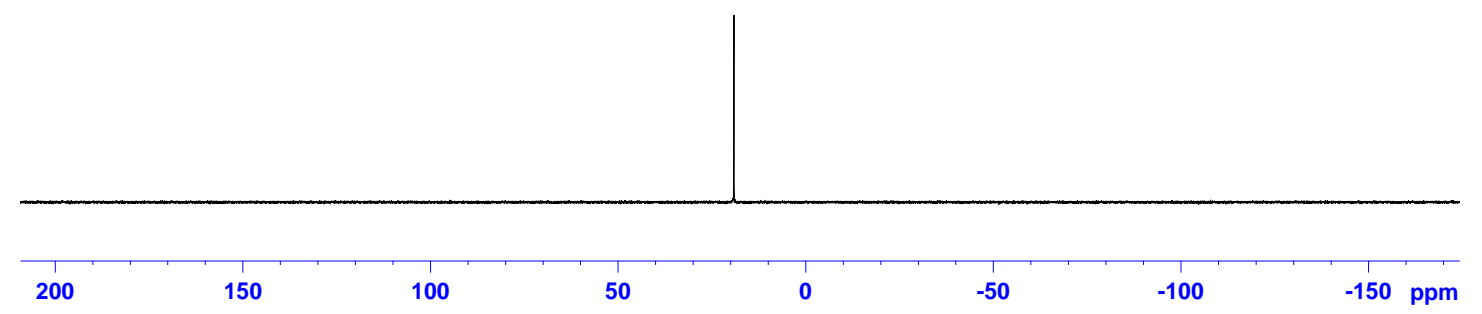


Methyl 3-(4-bromophenyl)-3-oxo-2-(triphenylphosphoranylidene)propanoate 7:

${ }^{1}$ H NMR (600 MHz, CDCl 3$)$
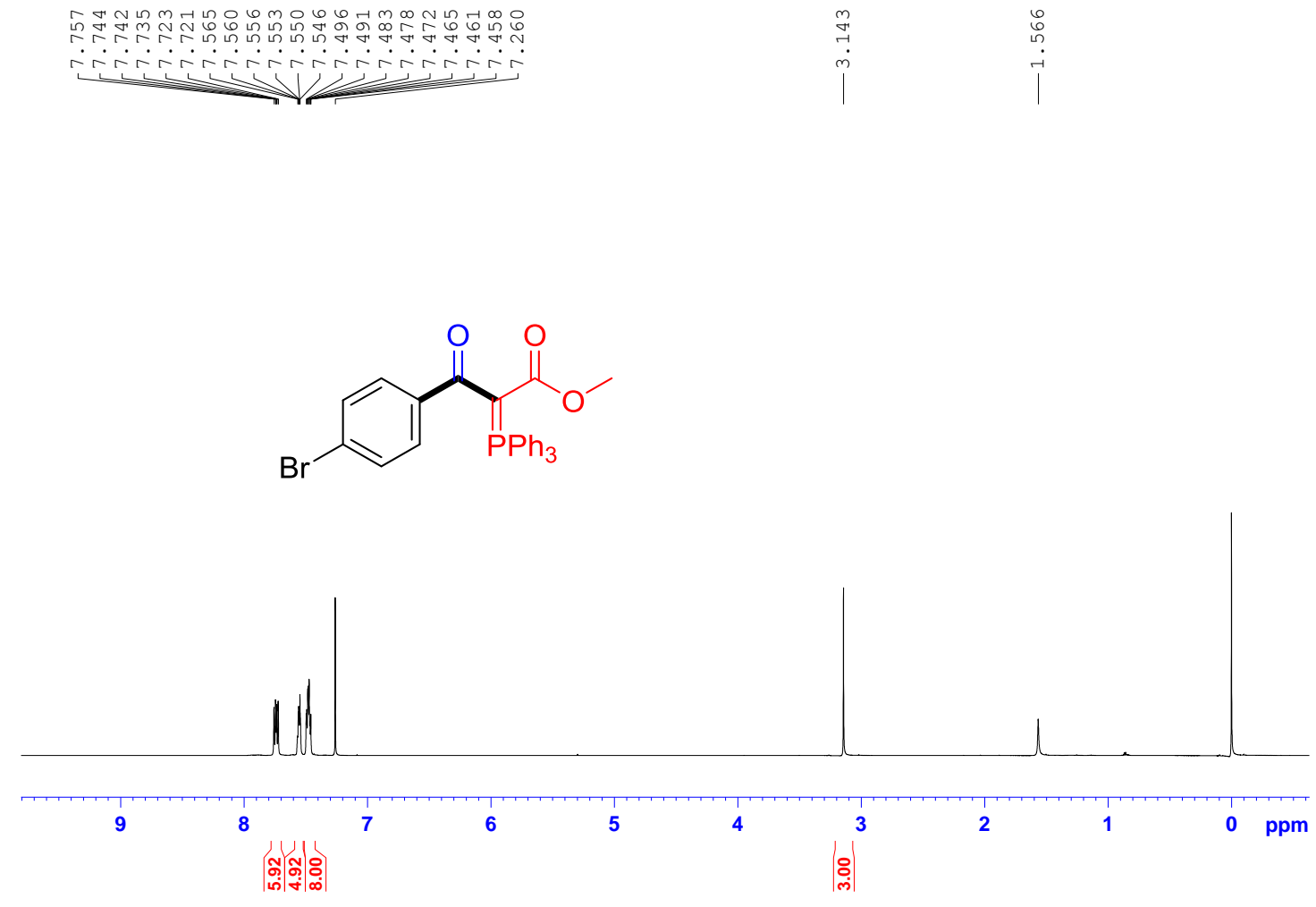

${ }^{13} \mathrm{C}$ NMR (100 MHz, $\left.\mathrm{CDCl}_{3}\right)$

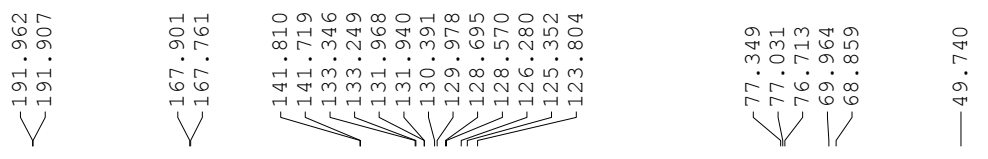

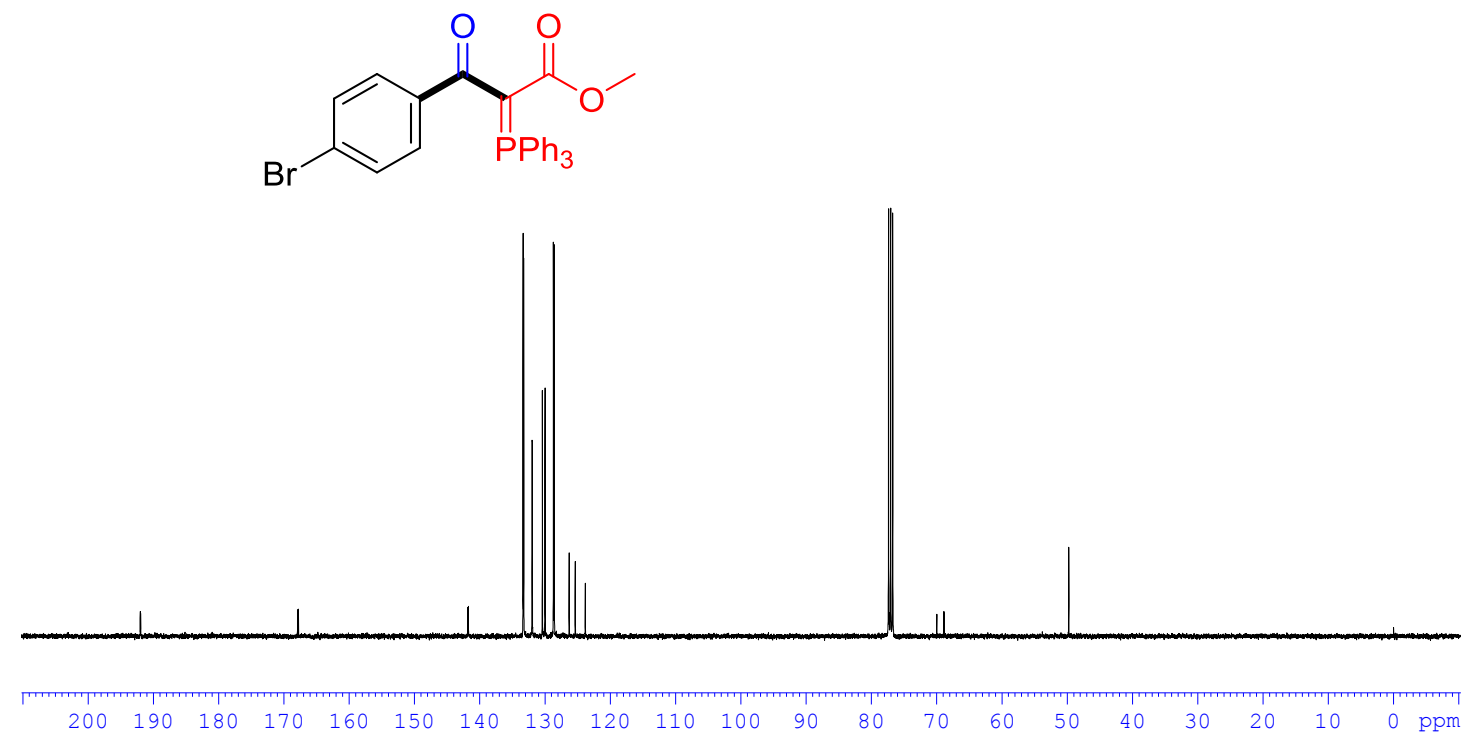


${ }^{31}$ P NMR (162 MHz, $\left.\mathrm{CDCl}_{3}\right)$<smiles>COC(=O)C(=Pc1ccccc1)C(=O)c1ccc(Br)cc1</smiles>

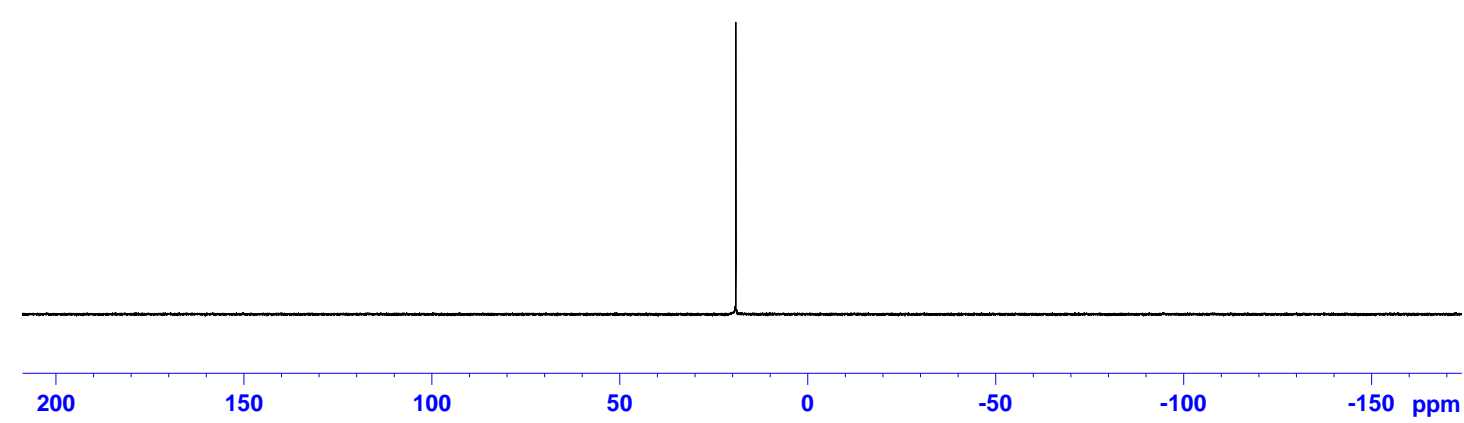

Methyl 3-(4-nitrophenyl)-3-oxo-2-(triphenylphosphoranylidene)propanoate 8:

${ }^{1} \mathrm{H}$ NMR (400 MHz, $\left.\mathrm{CDCl}_{3}\right)$

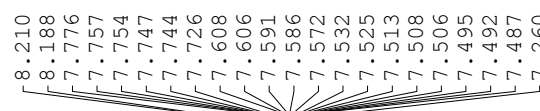<smiles>COC(=O)C(=P)C(=O)c1ccc([N+](=O)[O-])cc1</smiles>

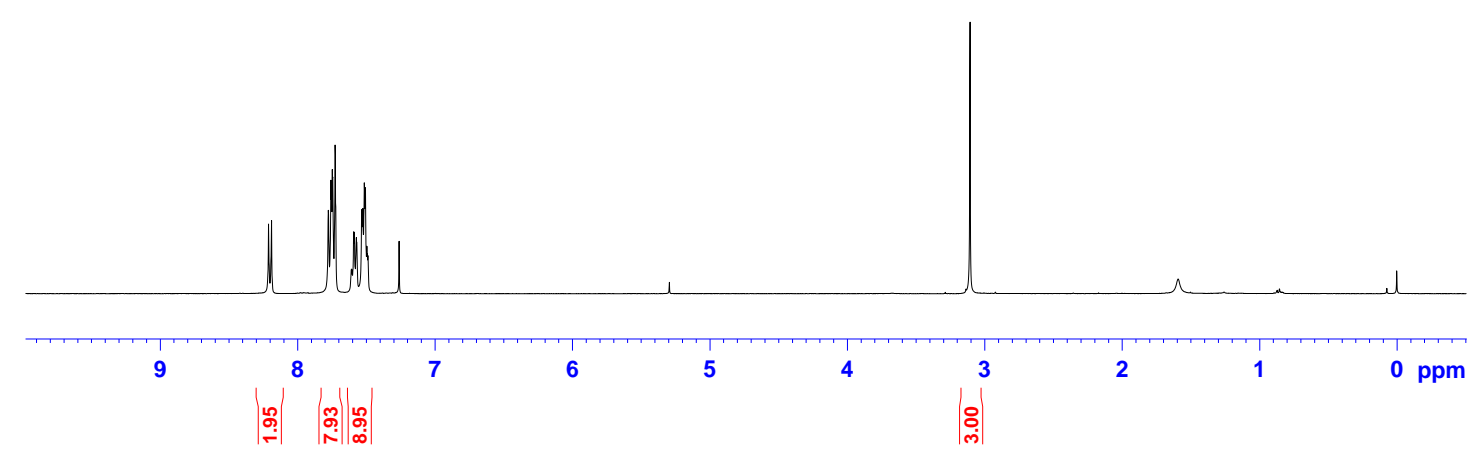


${ }^{13} \mathrm{C}$ NMR (100 MHz, $\left.\mathrm{CDCl}_{3}\right)$

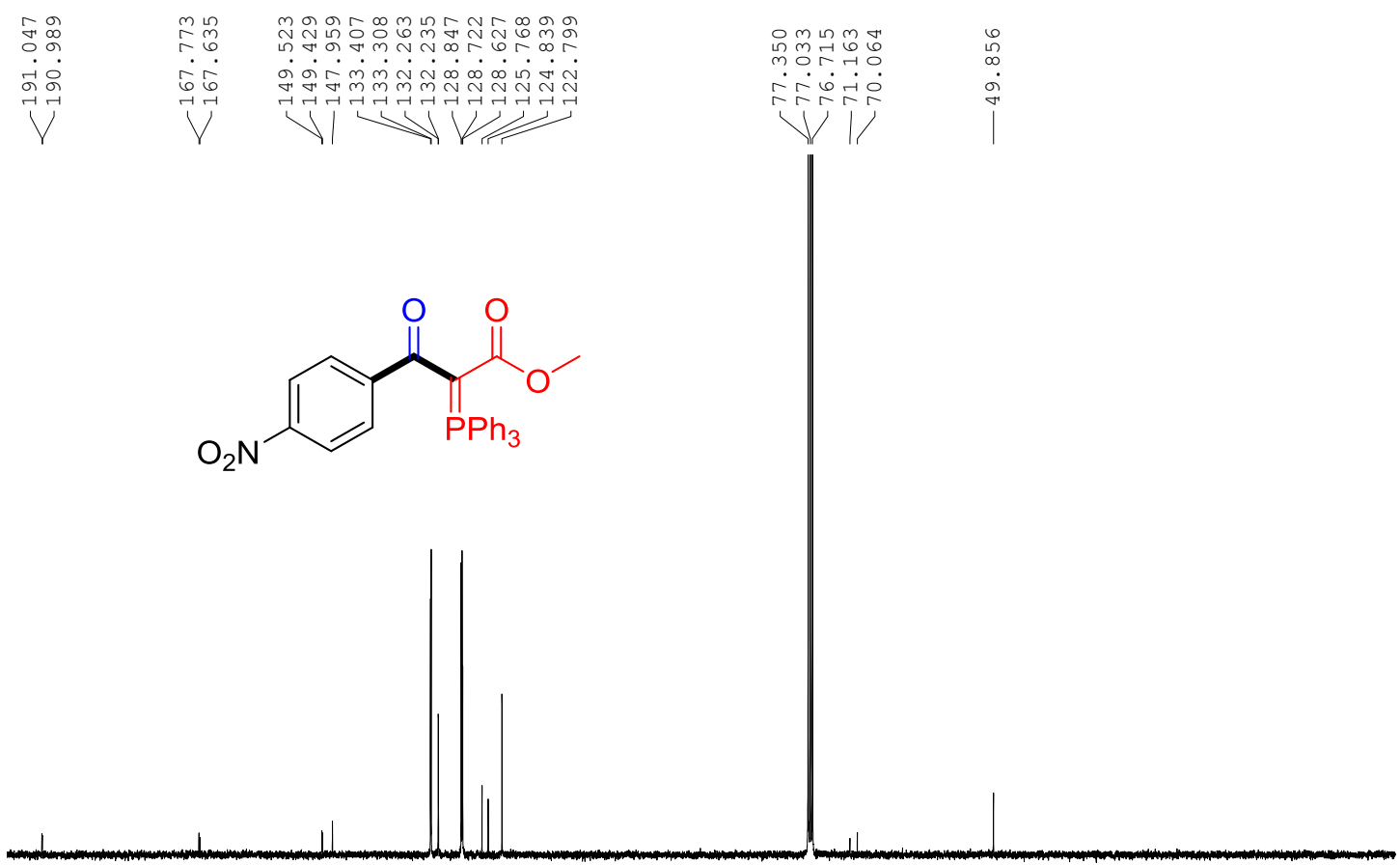

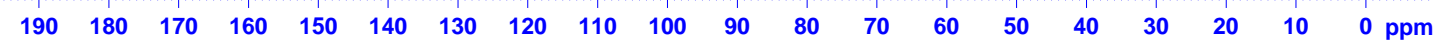
${ }^{31}$ P NMR (162 MHz, $\left.\mathrm{CDCl}_{3}\right)$

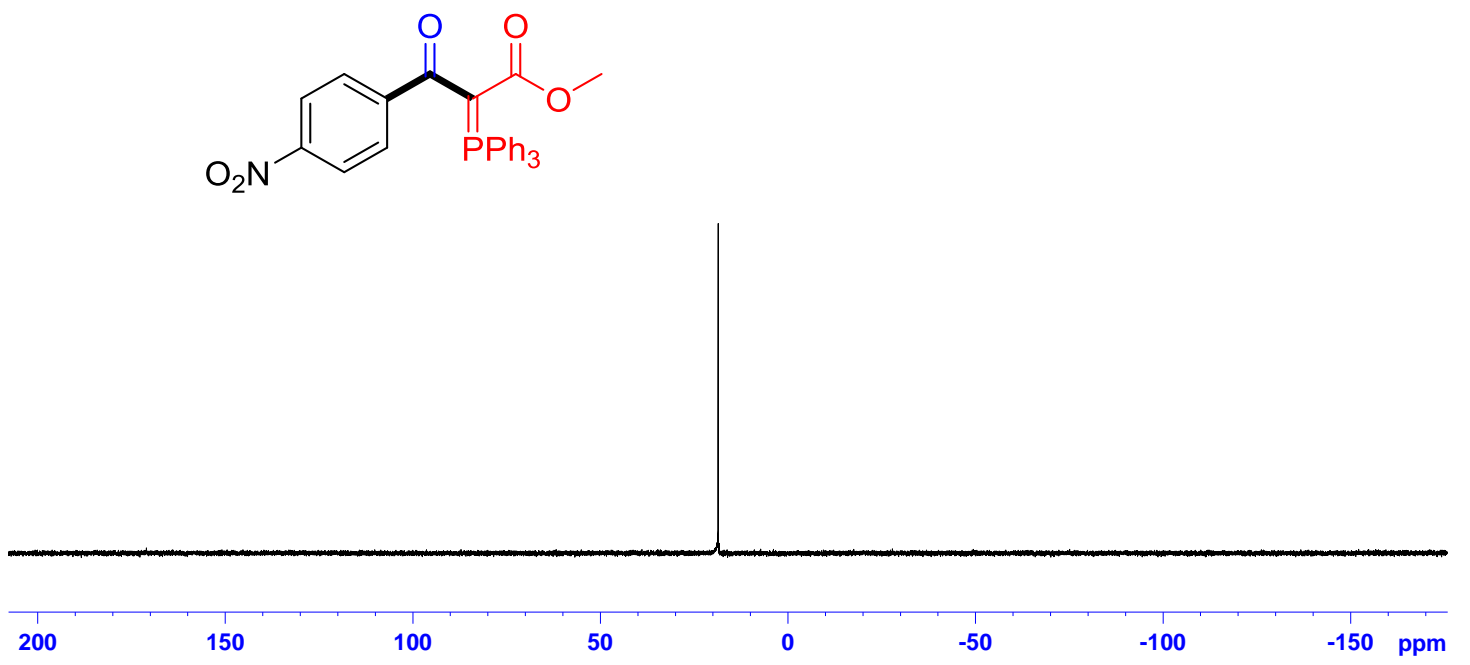


Methyl-3-oxo-3-(4-(trifluoromethyl)phenyl)-2-(triphenylphosphoranylidene)prop -anoate 9: ${ }^{1} \mathrm{H}$ NMR (400 MHz, $\left.\mathrm{CDCl}_{3}\right)$

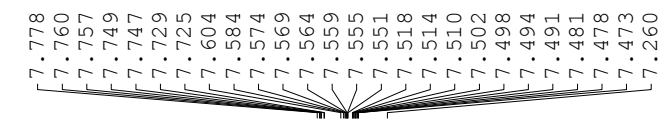<smiles>COC(=O)C(=Pc1ccccc1)C(=O)c1ccc(C(F)(F)F)cc1</smiles>

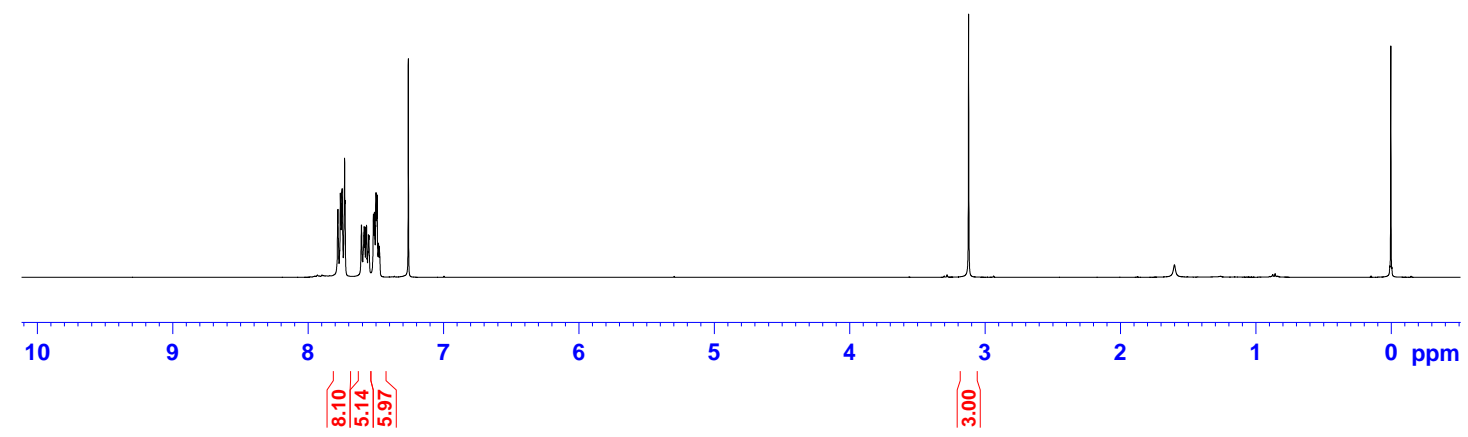

${ }^{13} \mathrm{C}$ NMR (100 MHz, $\left.\mathrm{CDCl}_{3}\right)$

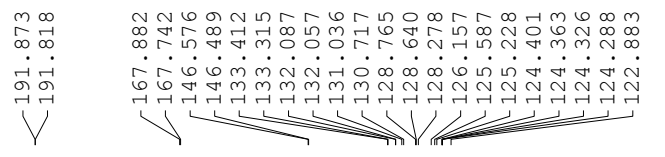<smiles>COC(=O)C(P=Pc1ccccc1)C(=O)c1ccc(C(F)(F)F)cc1</smiles>

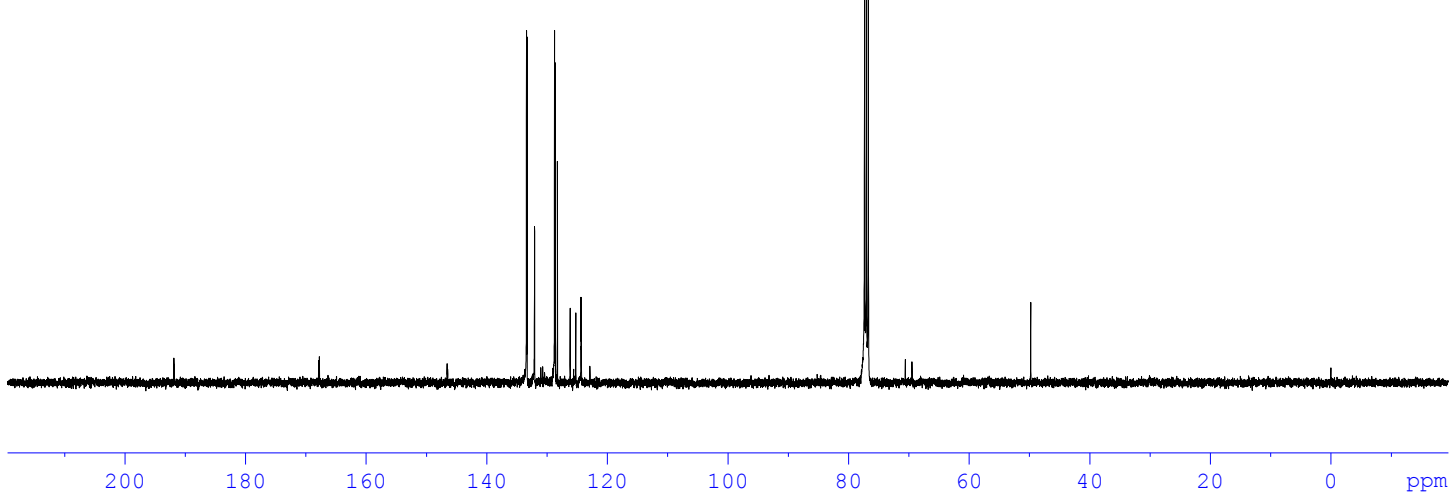


${ }^{31}$ P NMR (162 MHz, $\left.\mathrm{CDCl}_{3}\right)$<smiles>COC(=O)C(=Pc1ccccc1)C(=O)c1ccc(C(F)(F)F)cc1</smiles>

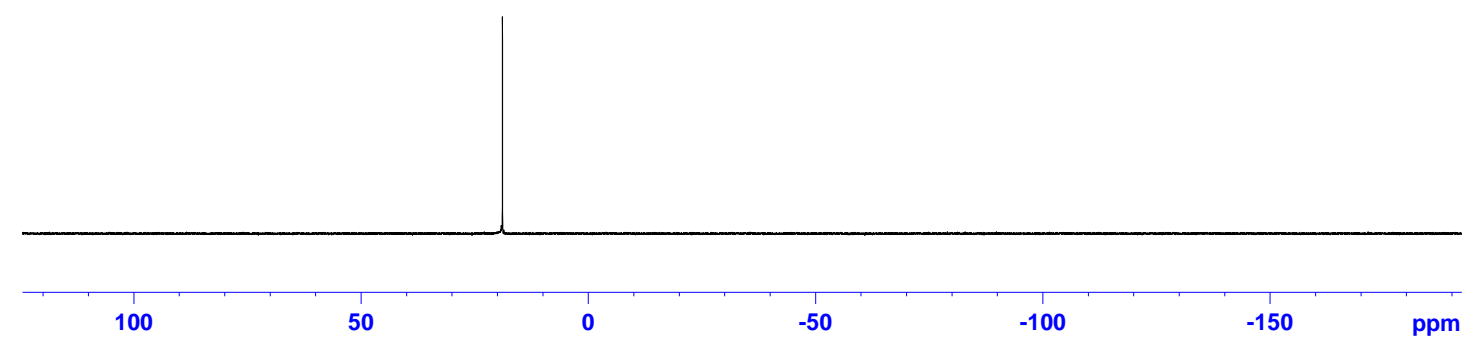

Methyl 3-oxo-3-(p-tolyl)-2-(triphenylphosphoranylidene)propanoate 10:

${ }^{1}$ H NMR (400 MHz, $\mathrm{CDCl}_{3}$ )
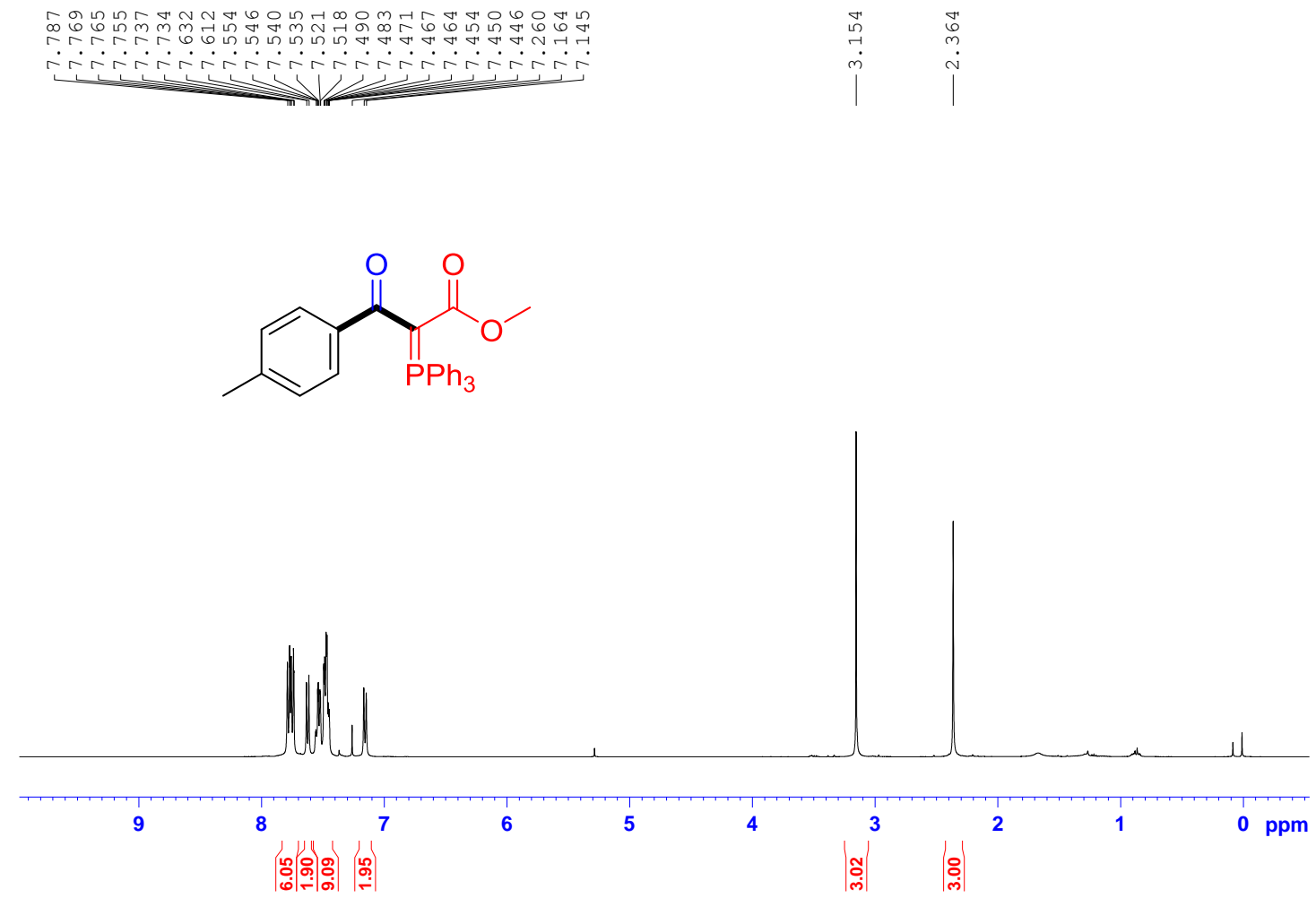


\section{${ }^{13} \mathrm{C}$ NMR (100 MHz, $\left.\mathrm{CDCl}_{3}\right)$}
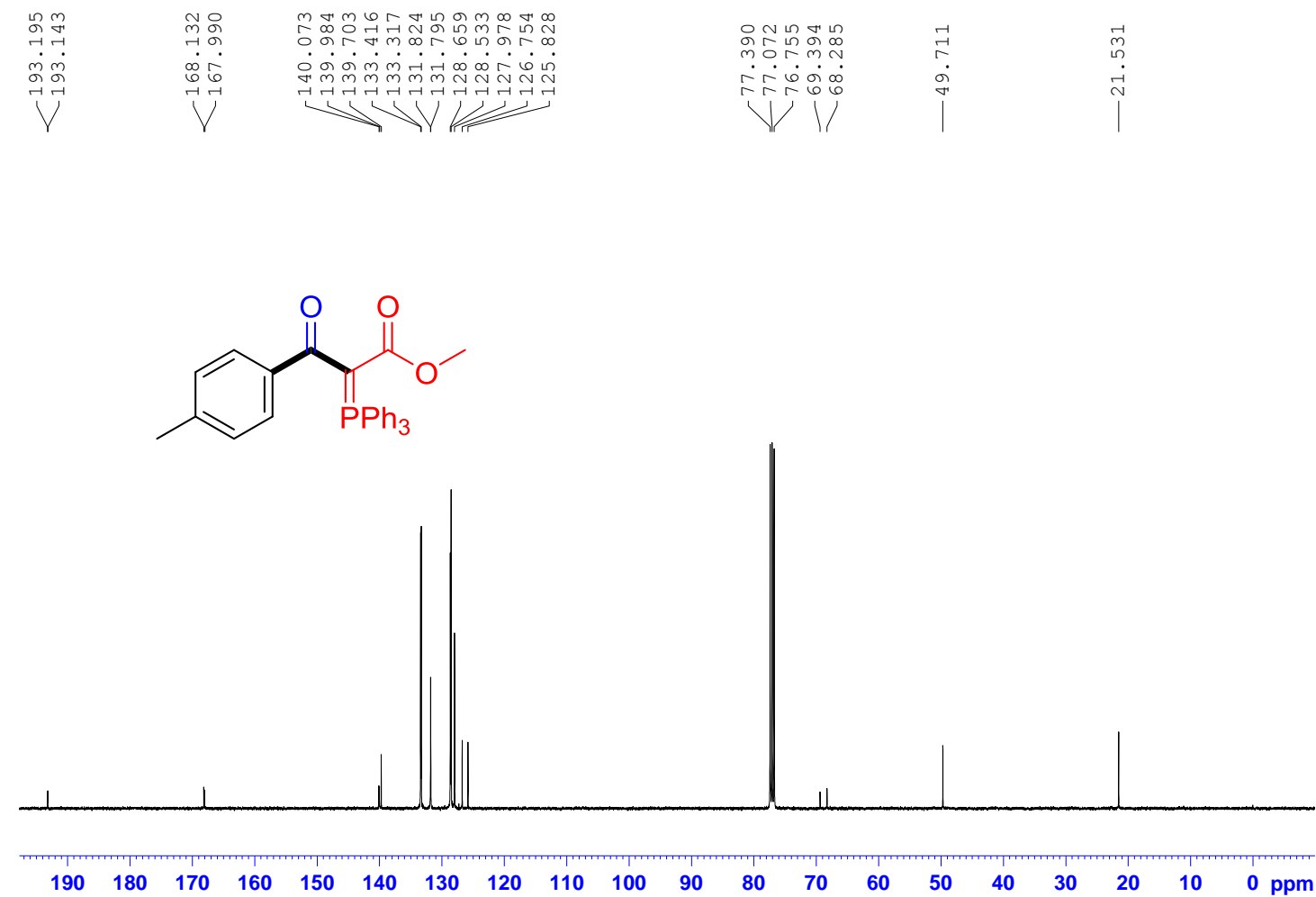

${ }^{31}$ P NMR (162 MHz, $\mathrm{CDCl}_{3}$ )

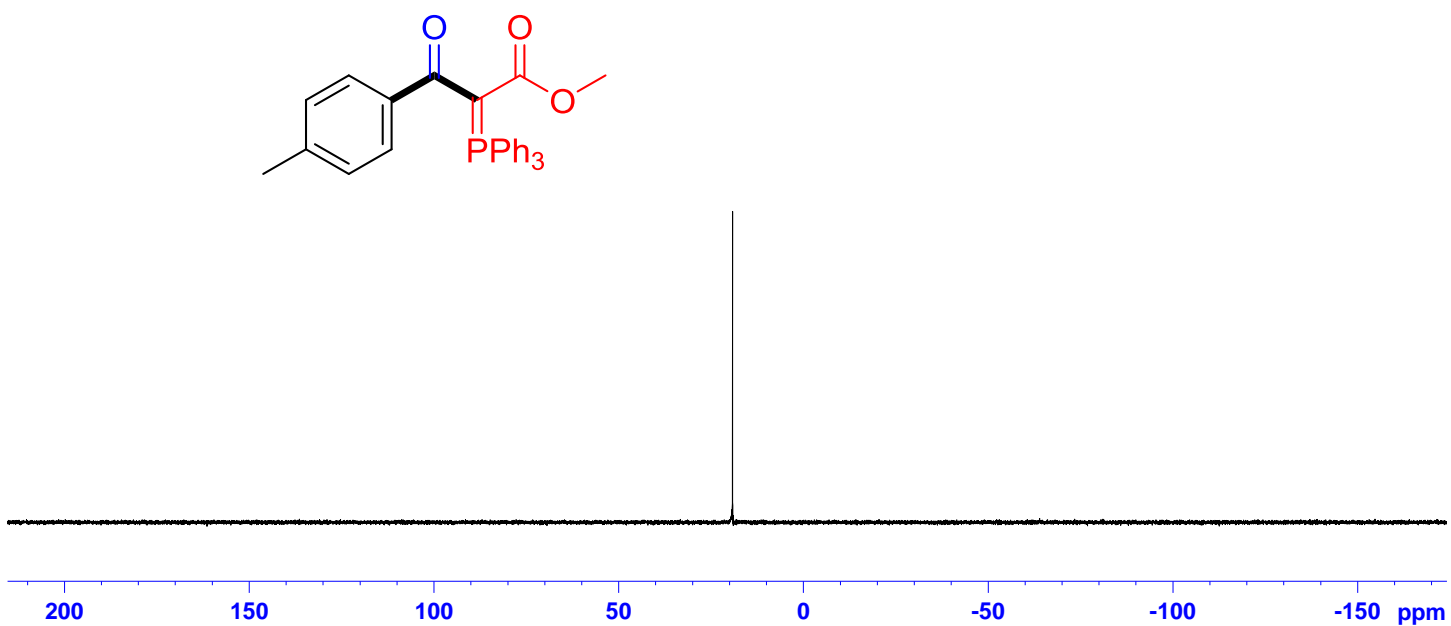


Methyl 3-(4-methoxyphenyl)-3-oxo-2-(triphenylphosphoranylidene)propanoate 11: ${ }^{1} \mathrm{H}$ NMR (400 $\left.\mathrm{MHz}, \mathrm{CDCl}_{3}\right)$

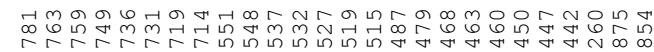

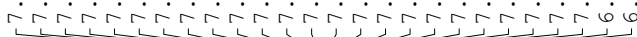<smiles>COC(=O)C(=P)C(=O)c1ccc(OC)cc1</smiles>

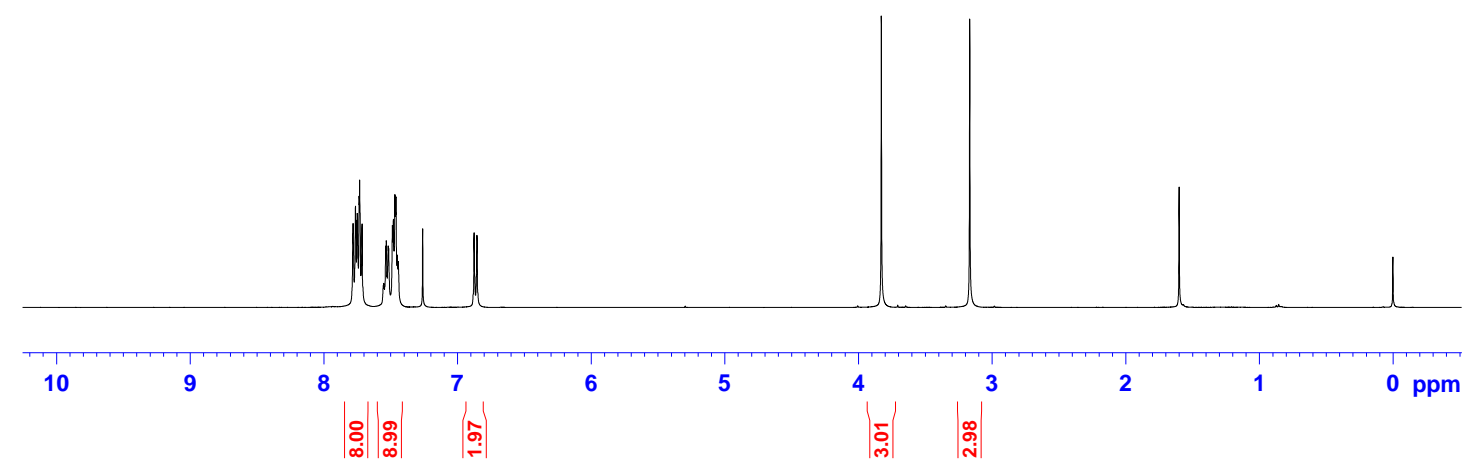

${ }^{13} \mathrm{C}$ NMR (100 MHz, $\left.\mathrm{CDCl}_{3}\right)$

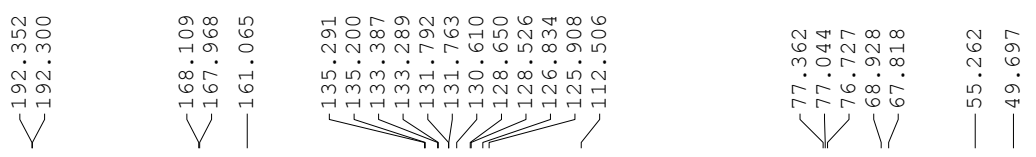<smiles>COC(=O)C(=P)C(=O)c1ccc(OC)cc1</smiles>

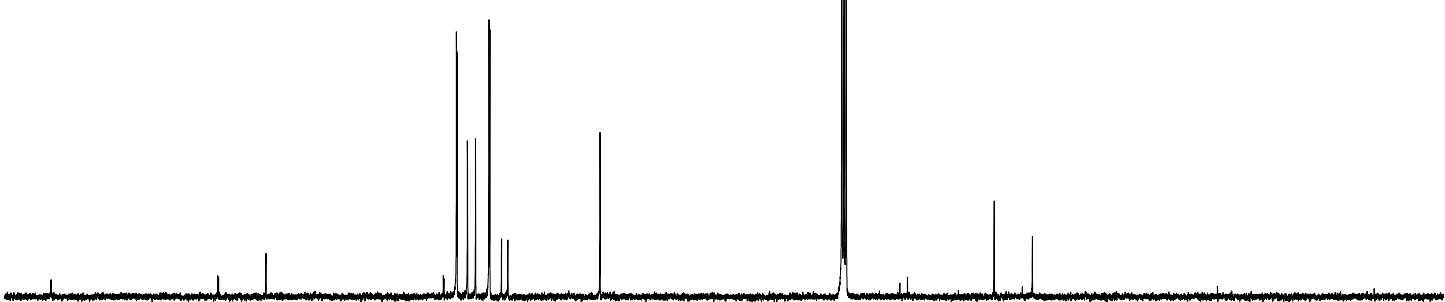

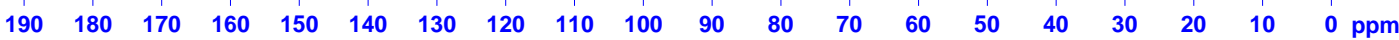


${ }^{31}$ P NMR (162 MHz, $\left.\mathrm{CDCl}_{3}\right)$<smiles>COC(=O)C(C(=O)c1ccccc1)C(=O)c1ccc(OC)cc1</smiles>

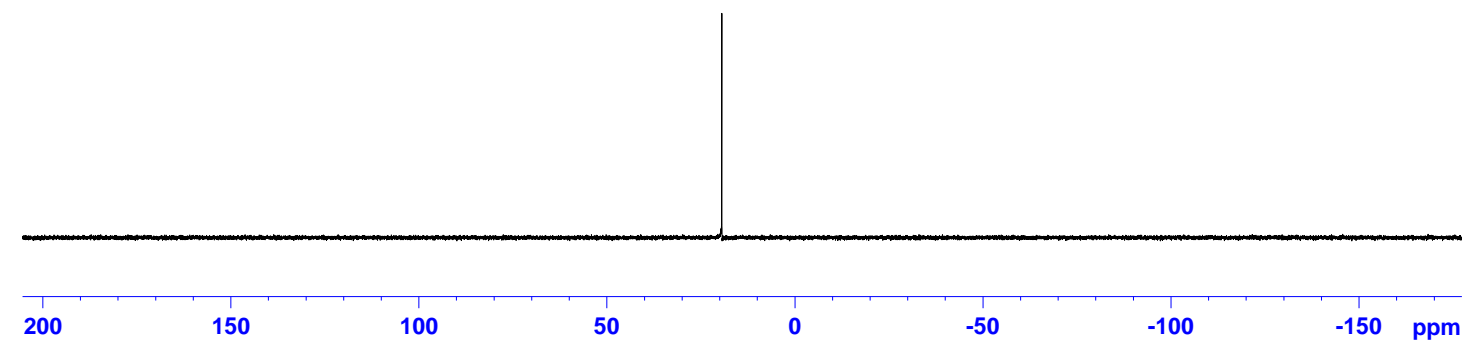

Methyl-3-(4-(tert-butyl)phenyl)-3-oxo-2-(triphenylphosphoranylidene)propanoat -e 12: ${ }^{1} \mathrm{H}$ NMR (400 MHz, $\left.\mathrm{CDCl}_{3}\right)$<smiles>COC(=O)C(=P)C(=O)c1ccc(C(C)(C)C)cc1</smiles>

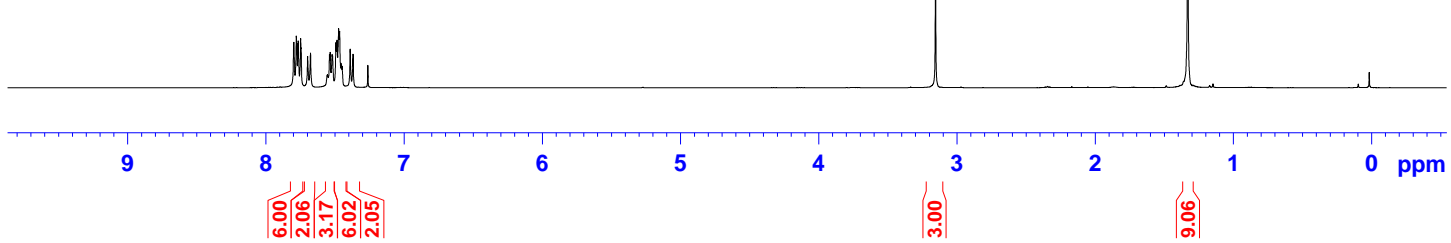




\section{${ }^{13} \mathrm{C}$ NMR (100 MHz, $\left.\mathrm{CDCl}_{3}\right)$}

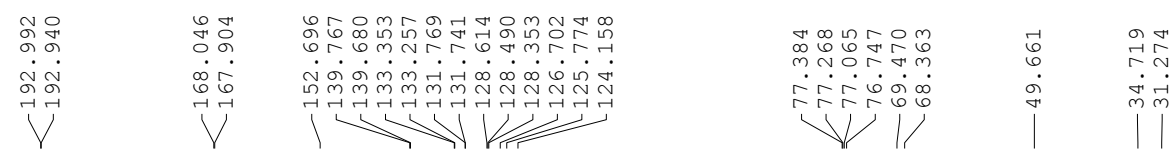<smiles>COC(=O)C(Pc1ccccc1)C(=O)c1ccc(C(C)(C)C)cc1</smiles>

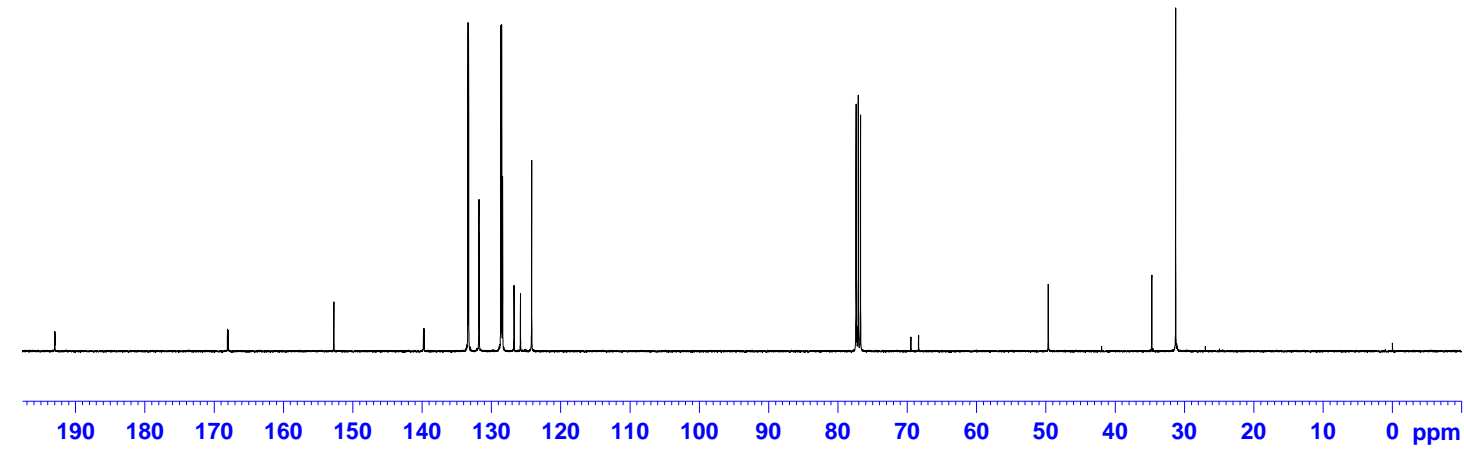

${ }^{31}$ P NMR (162 MHz, $\mathrm{CDCl}_{3}$ )<smiles>COC(=O)C(=Pc1ccccc1)C(=O)c1ccc(C(C)(C)C)cc1</smiles>

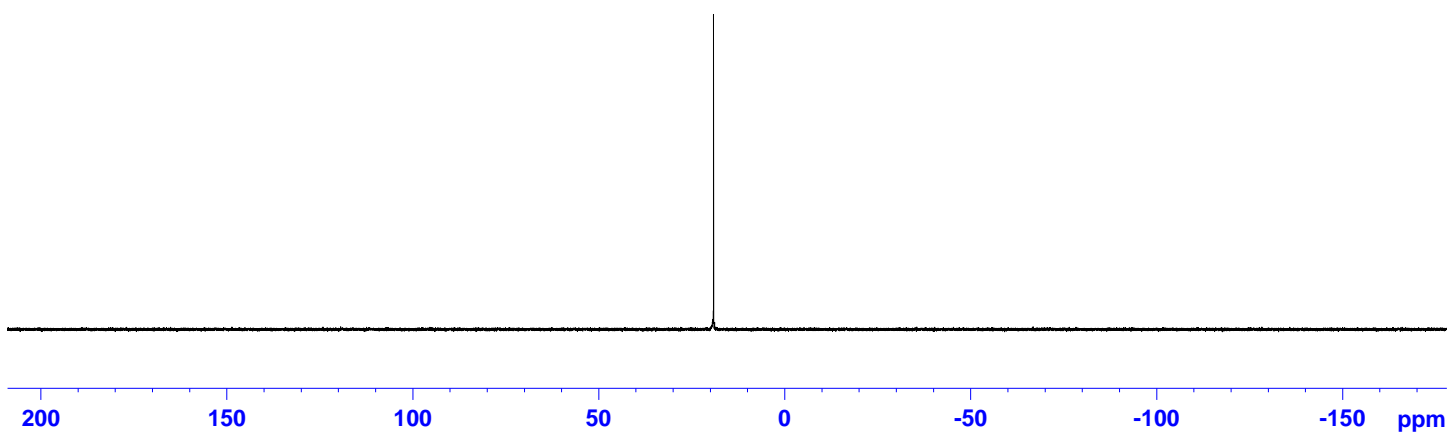


Methyl-3-(4-(1H-pyrrol-1-yl)phenyl)-3-oxo-2-(triphenylphosphoranylidene)propanoate 13: ${ }^{1} \mathrm{H}$ NMR (400 $\left.\mathrm{MHz}, \mathrm{CDCl}_{3}\right)$
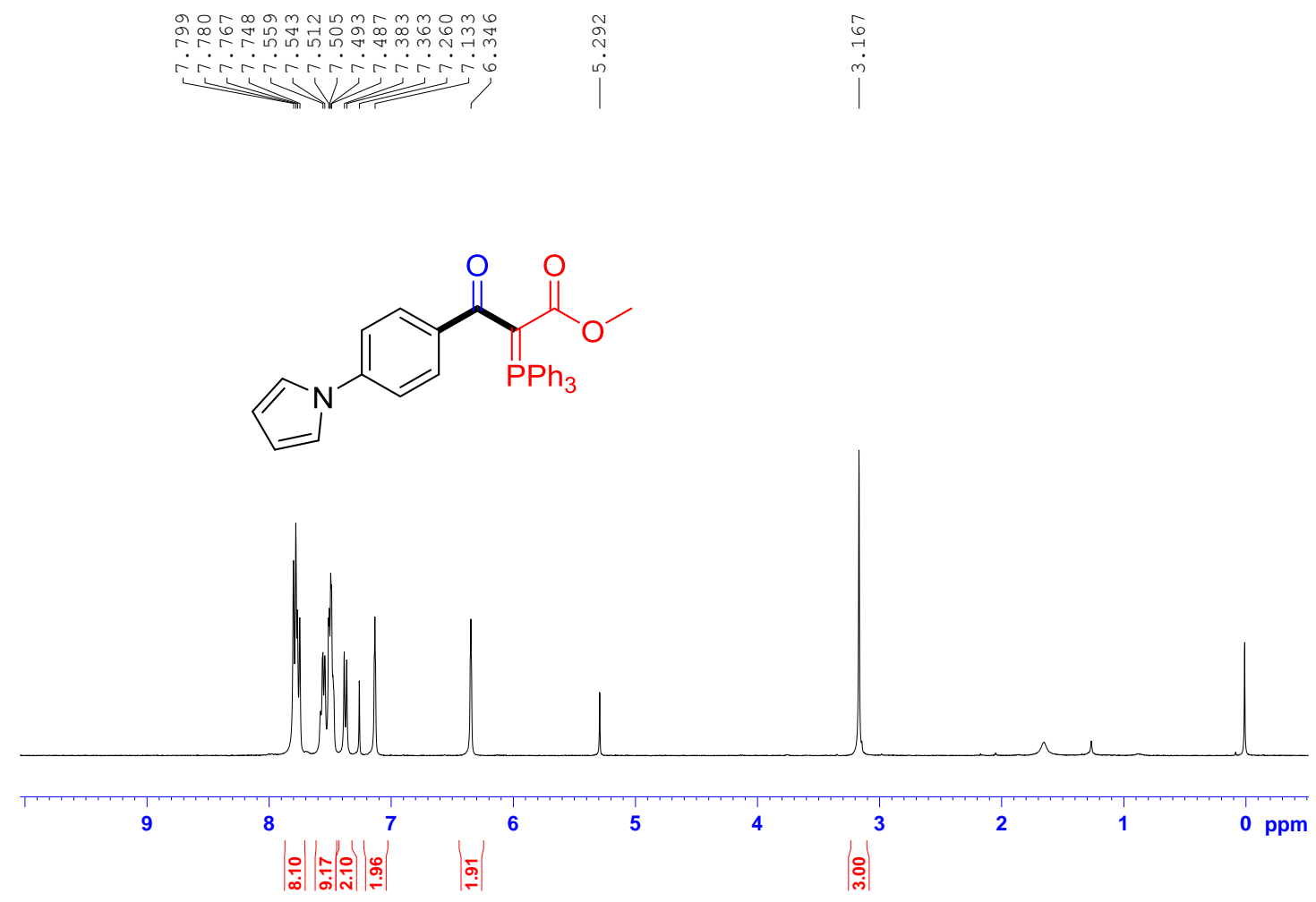

\section{${ }^{13} \mathrm{C}$ NMR (150 MHz, $\left.\mathrm{CDCl}_{3}\right)$}
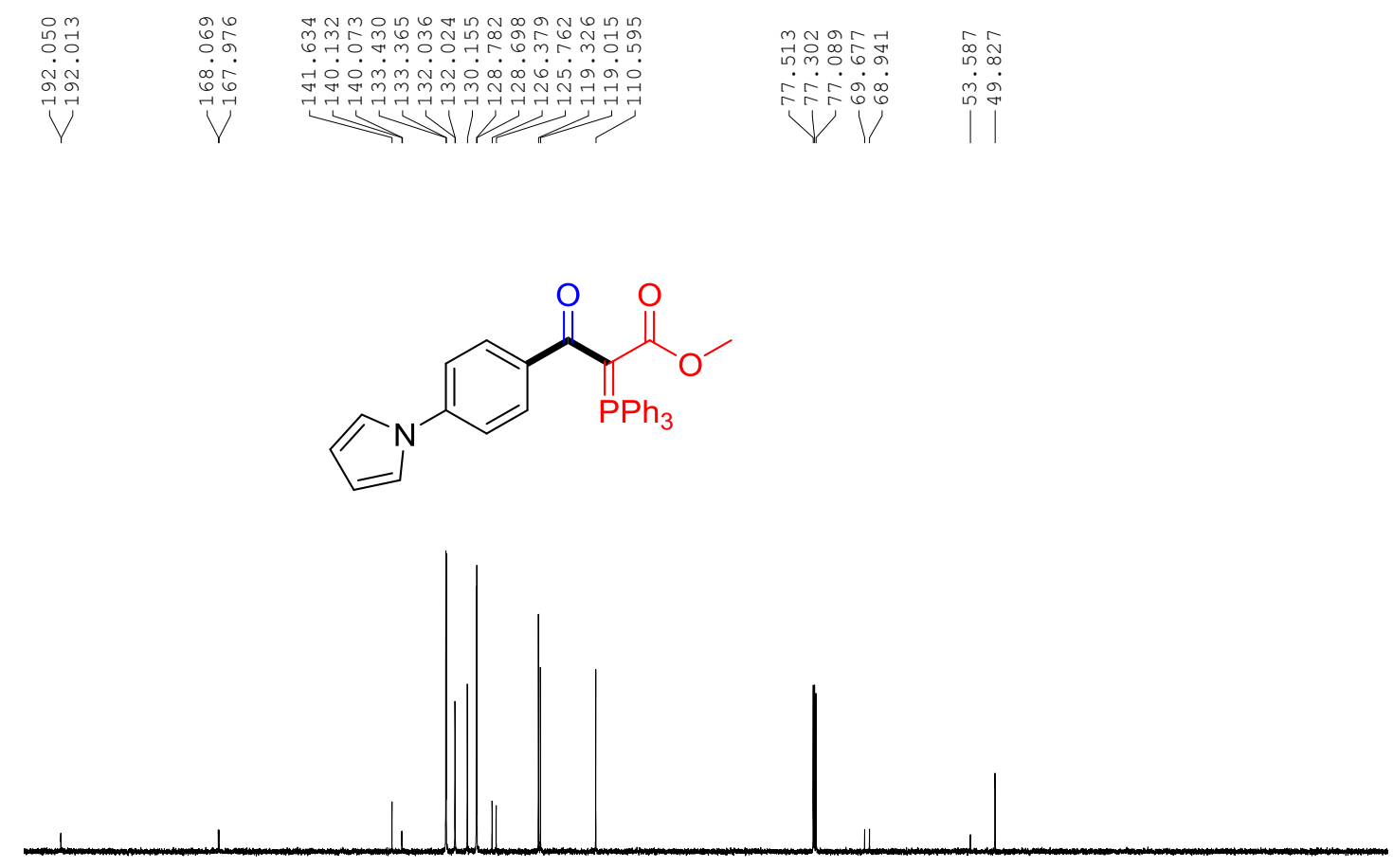

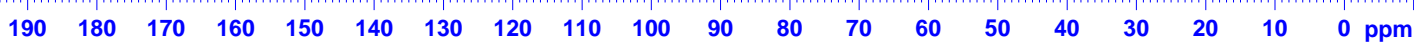


${ }^{31}$ P NMR (162 MHz, $\left.\mathrm{CDCl}_{3}\right)$

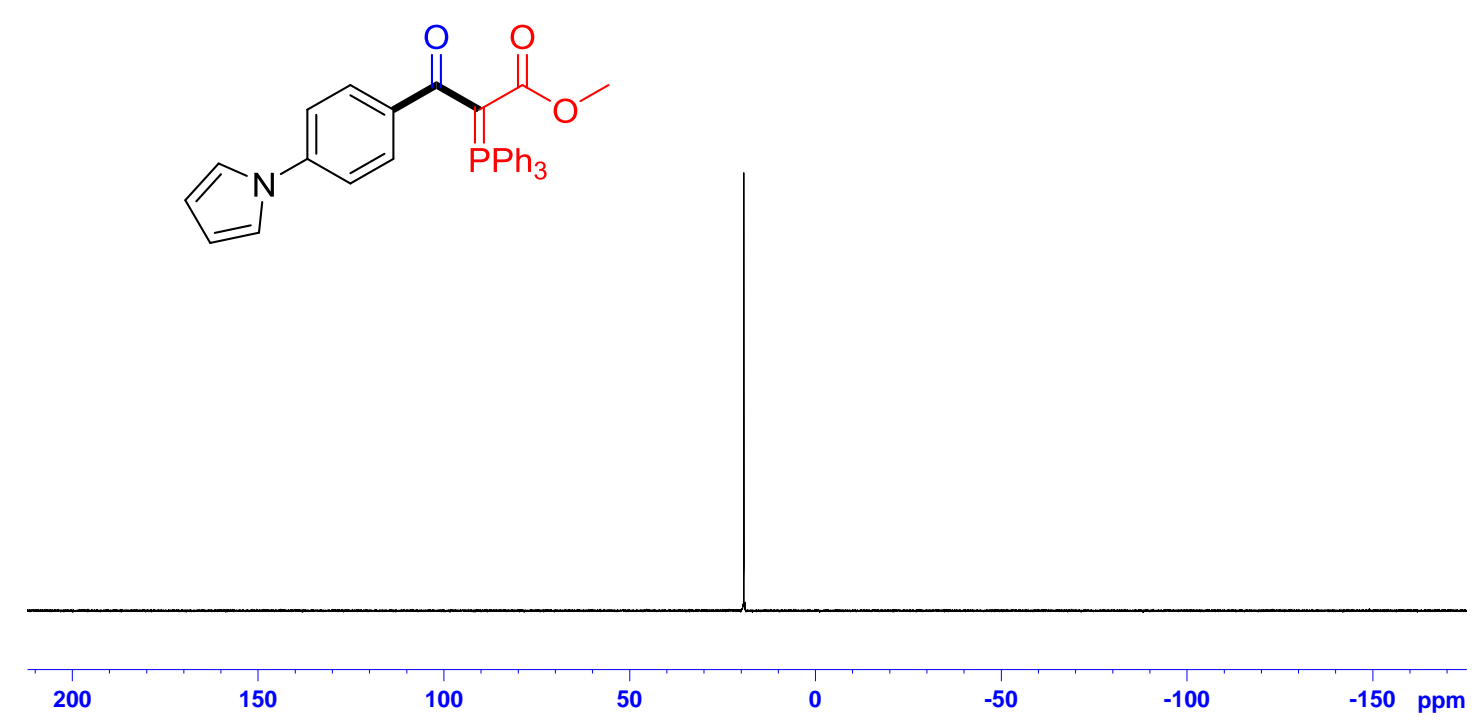

Methyl 3-oxo-3-phenyl-2-(triphenylphosphoranylidene)propanoate 14:

${ }^{1}$ H NMR (400 MHz, CDCl3)

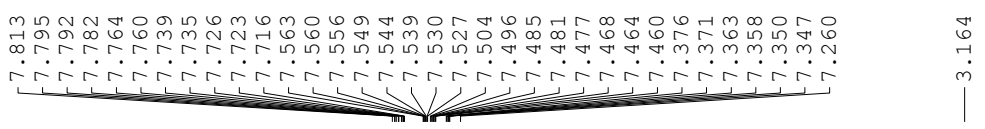

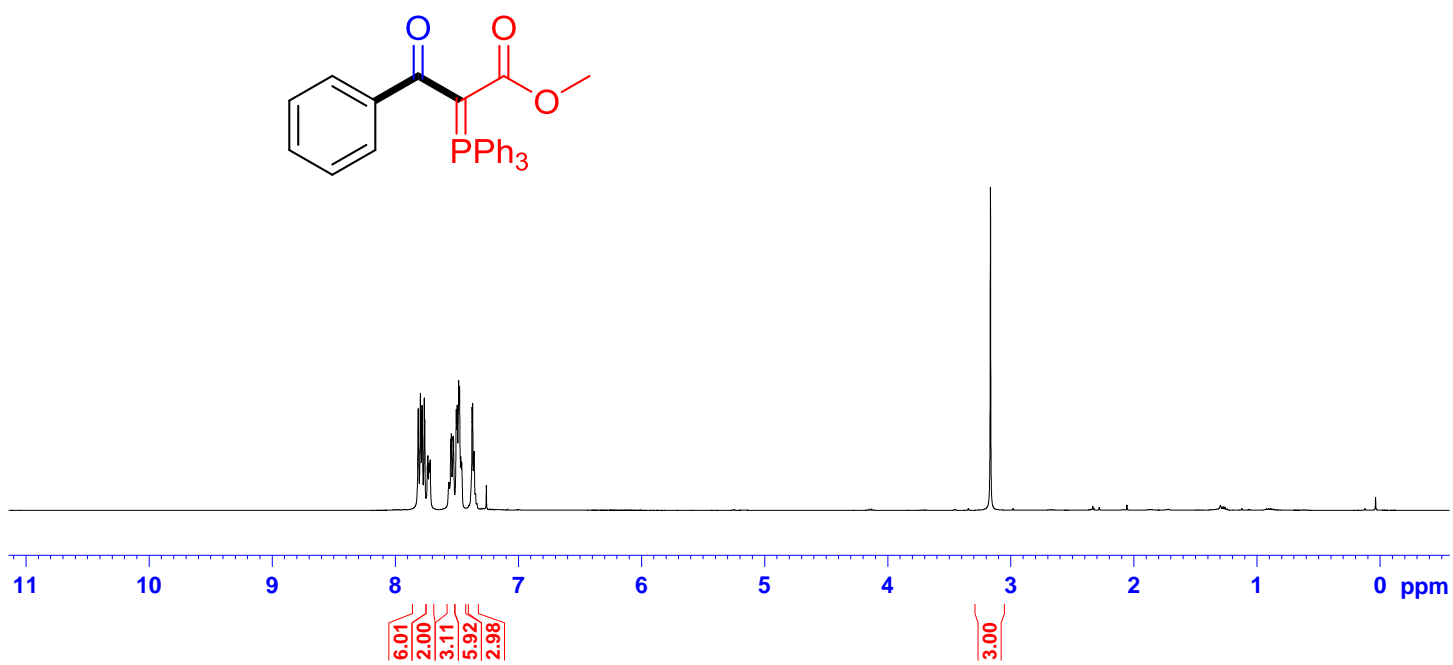




\section{${ }^{13}$ C NMR (100 MHz, $\left.\mathrm{CDCl}_{3}\right)$}

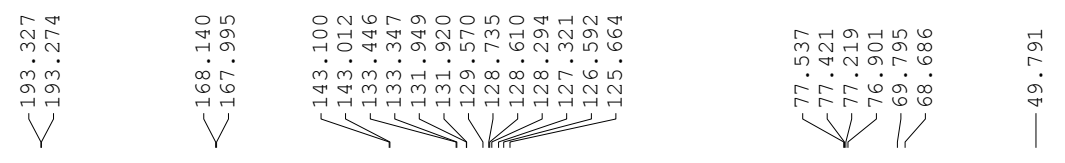<smiles>COC(=O)C(C(=O)c1ccccc1)P(c1ccccc1)c1ccccc1</smiles>

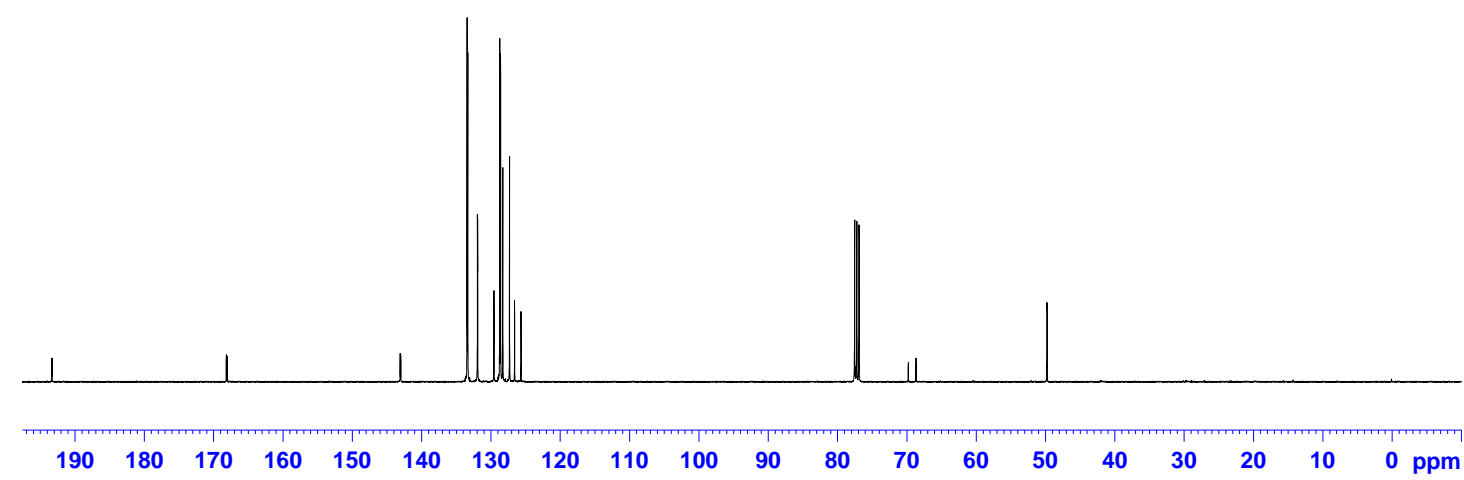

${ }^{31}$ P NMR (162 MHz, $\mathrm{CDCl}_{3}$ )<smiles>COC(=O)C(=Pc1ccccc1)C(=O)c1ccccc1</smiles>

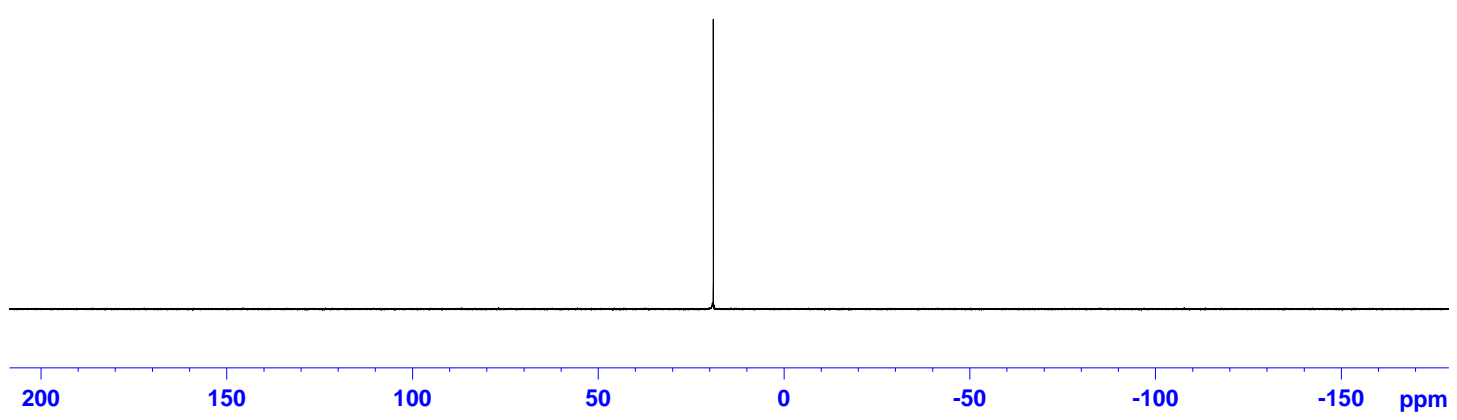


Methyl 3-(3-methoxy-3-oxo-2-(triphenylphosphoranylidene)propanoyl)benzoate 15: ${ }^{1} \mathrm{H}$ NMR (400 MHz, $\left.\mathrm{CDCl}_{3}\right)$

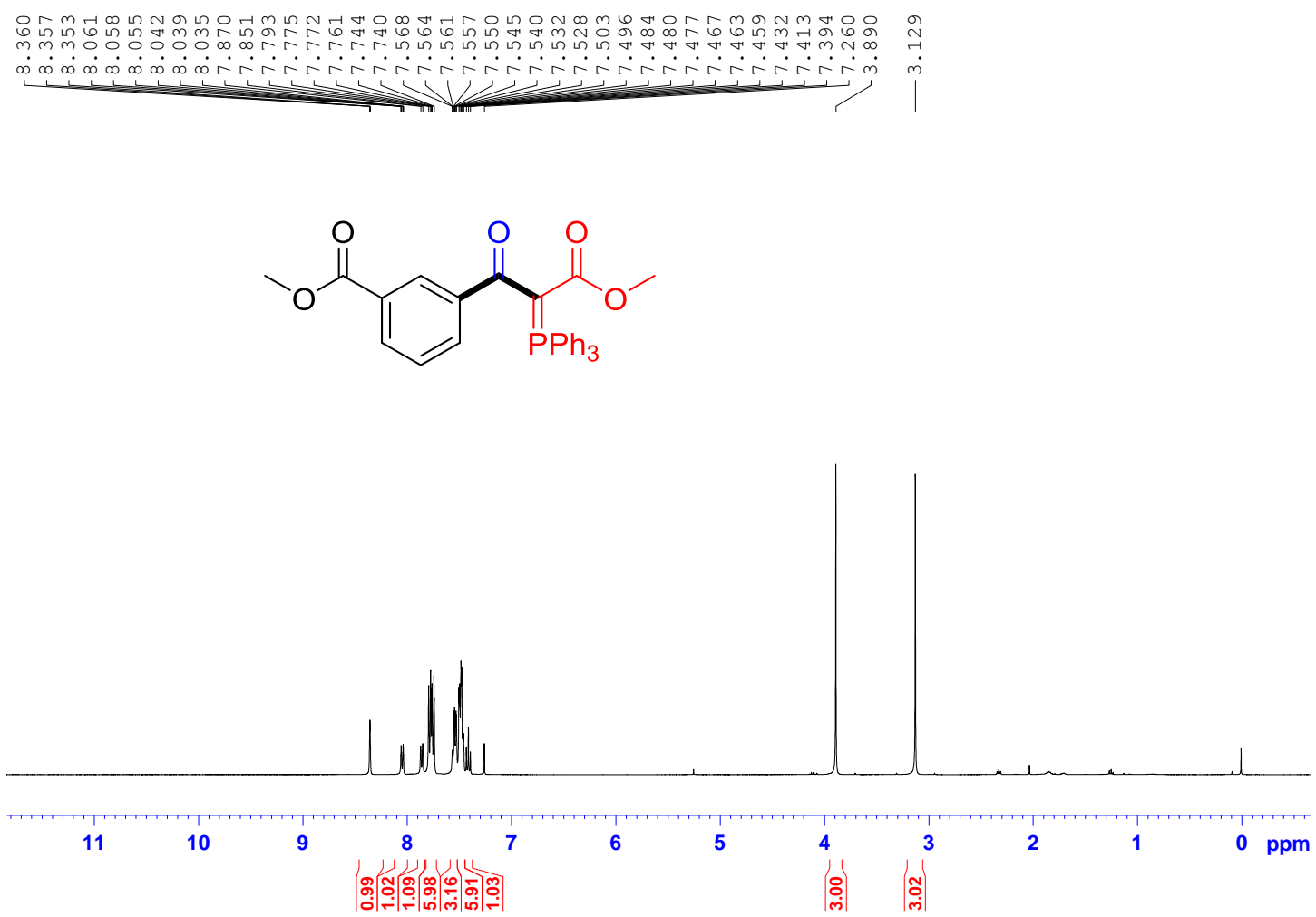

\section{${ }^{13} \mathrm{C}$ NMR (100 MHz, $\left.\mathrm{CDCl}_{3}\right)$}
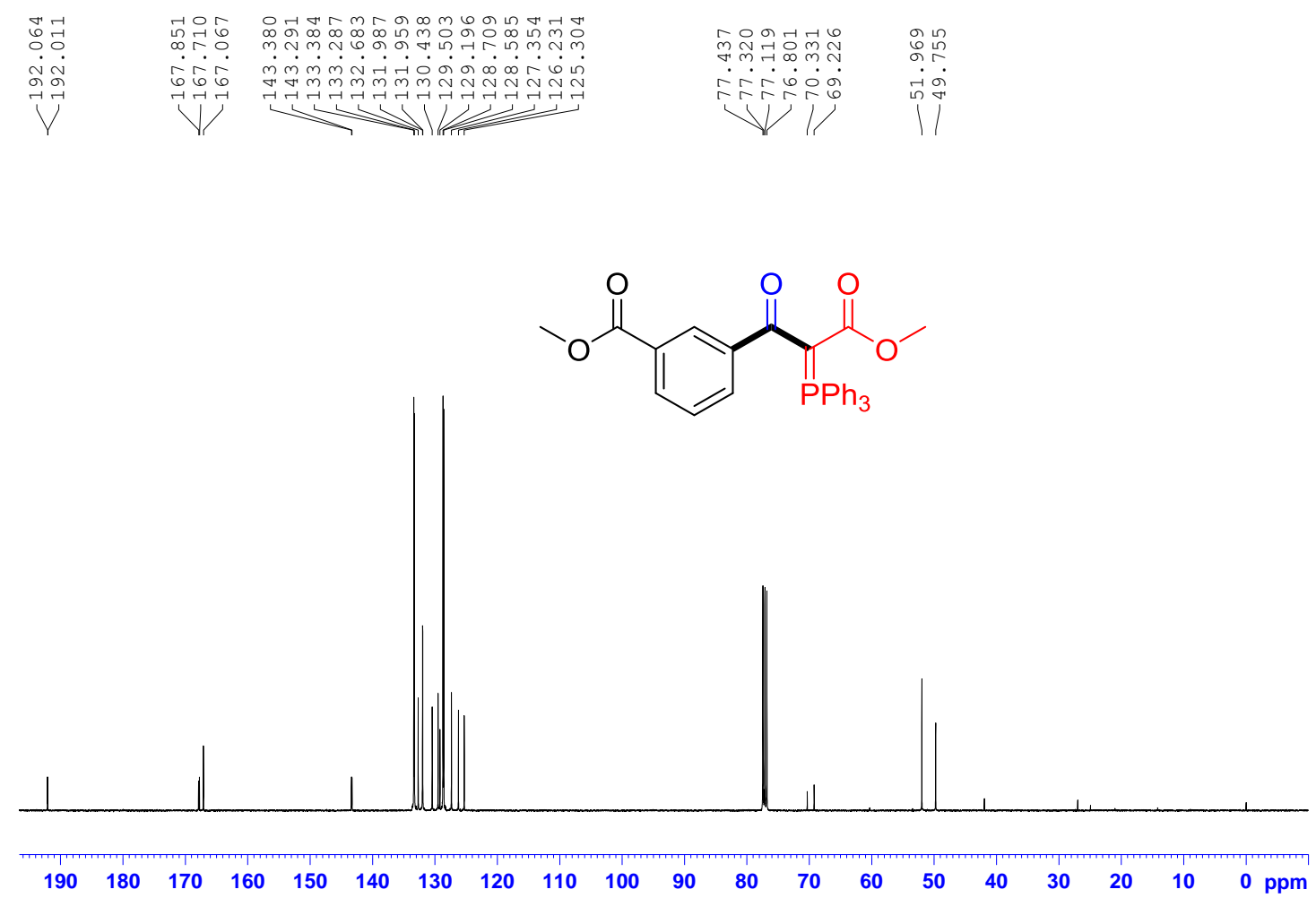
${ }^{31}$ P NMR (162 MHz, $\left.\mathrm{CDCl}_{3}\right)$

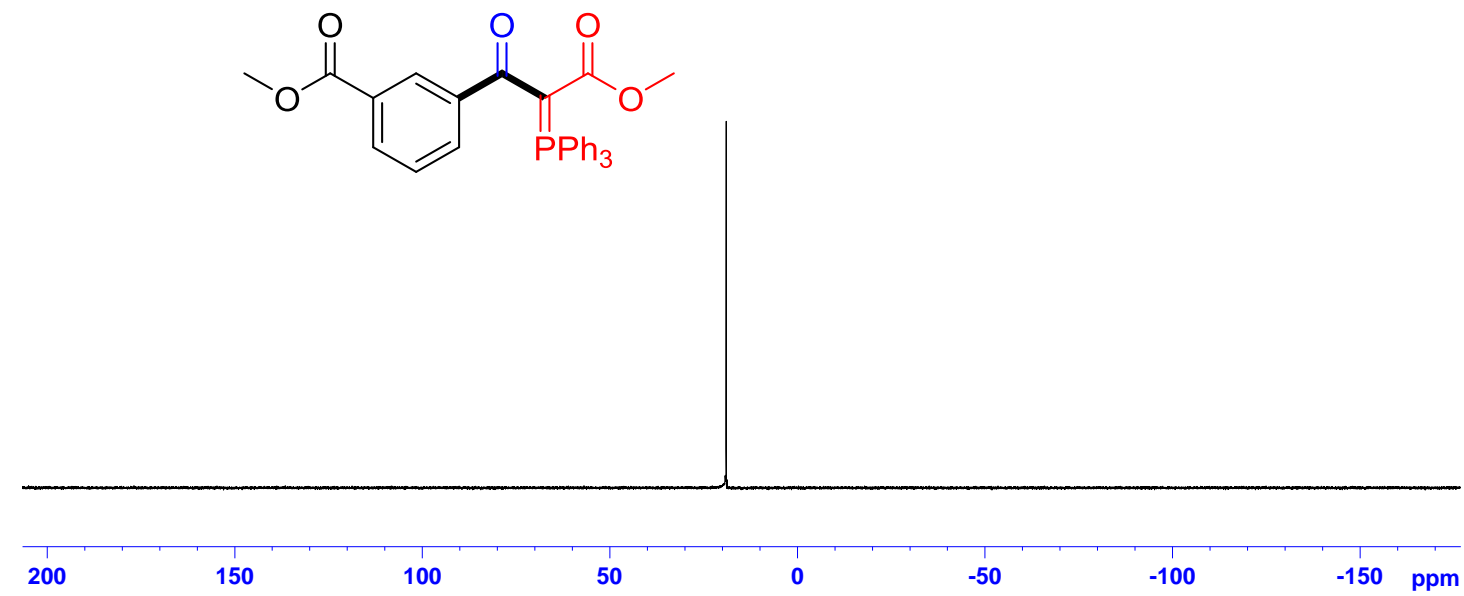

Methyl 3-(3-fluorophenyl)-3-oxo-2-(triphenylphosphoranylidene)propanoate 16:

${ }^{1} \mathrm{H}$ NMR (400 MHz, $\left.\mathrm{CDCl}_{3}\right)$

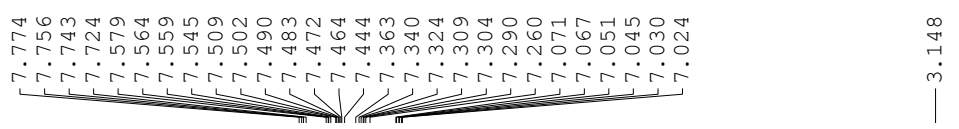<smiles>COC(=O)C(=Pc1ccccc1)C(=O)c1cccc(F)c1</smiles>

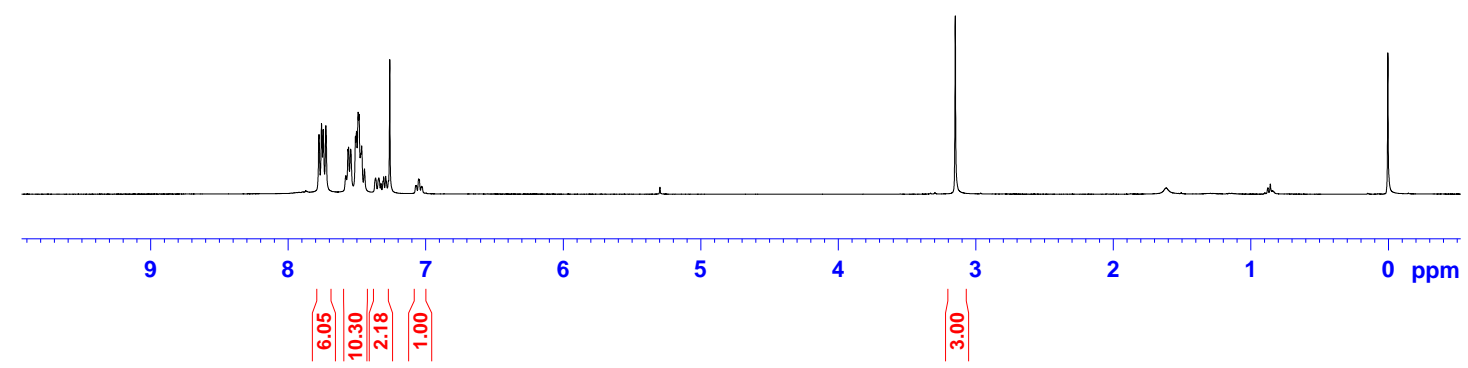


${ }^{13} \mathrm{C}$ NMR (100 MHz, $\left.\mathrm{CDCl}_{3}\right)$

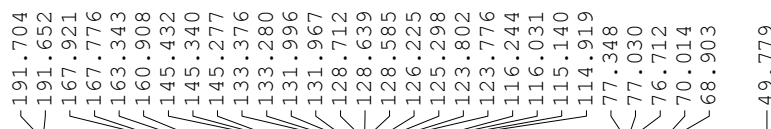

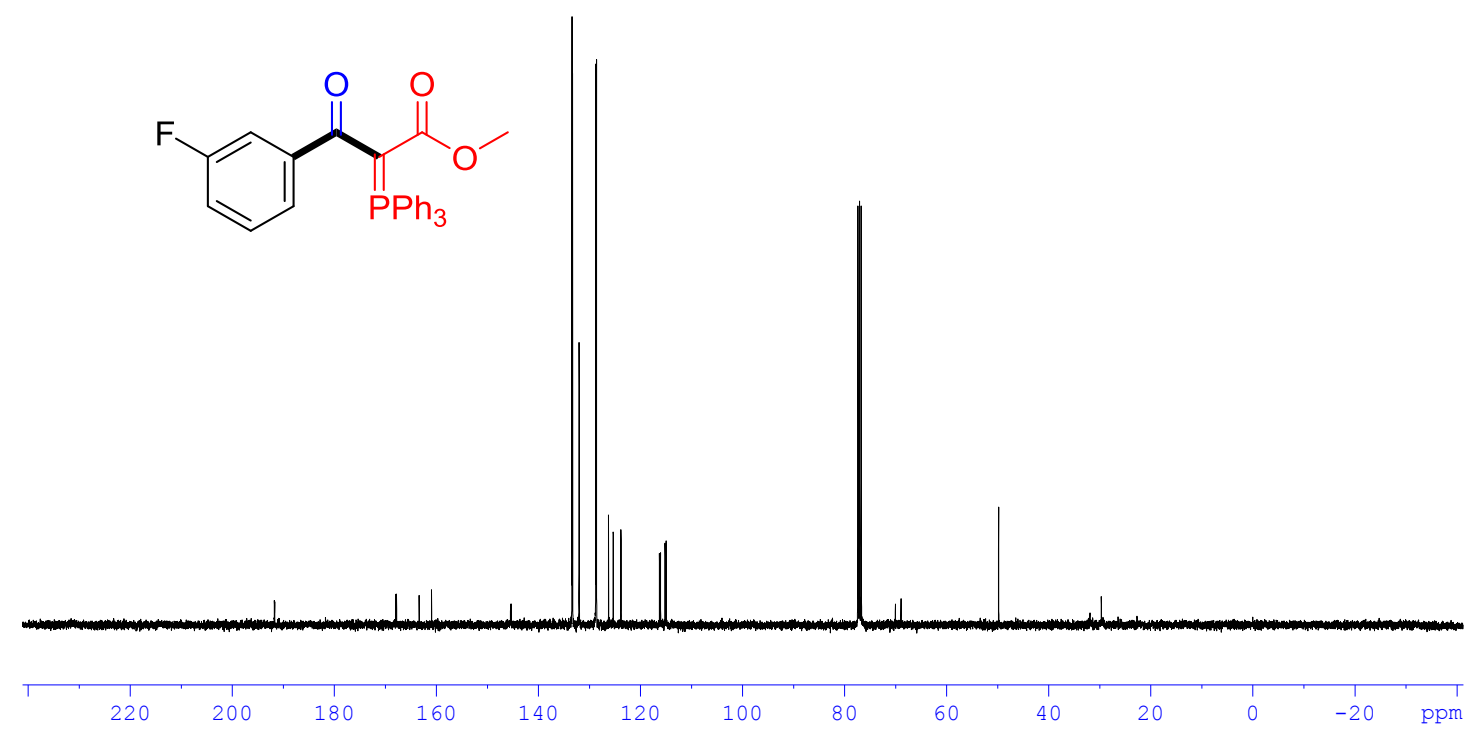

${ }^{31}$ P NMR (162 MHz, $\left.\mathrm{CDCl}_{3}\right)$

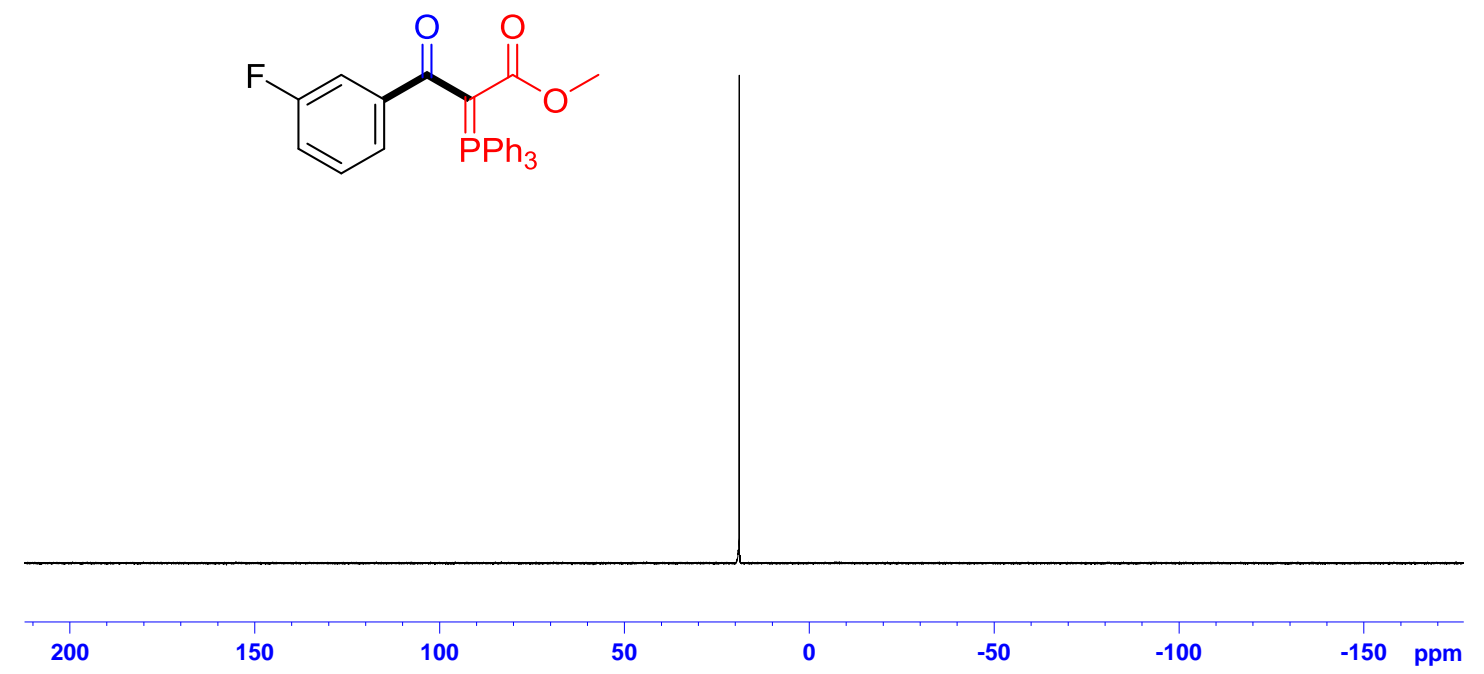


Methyl 3-(3-chlorophenyl)-3-oxo-2-(triphenylphosphoranylidene)propanoate 17:

${ }^{1}$ H NMR (400 MHz, CDCl 3$)$

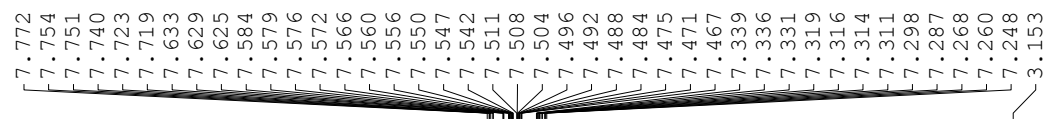<smiles>COC(=O)C(=P)C(=O)c1cccc(Cl)c1</smiles>

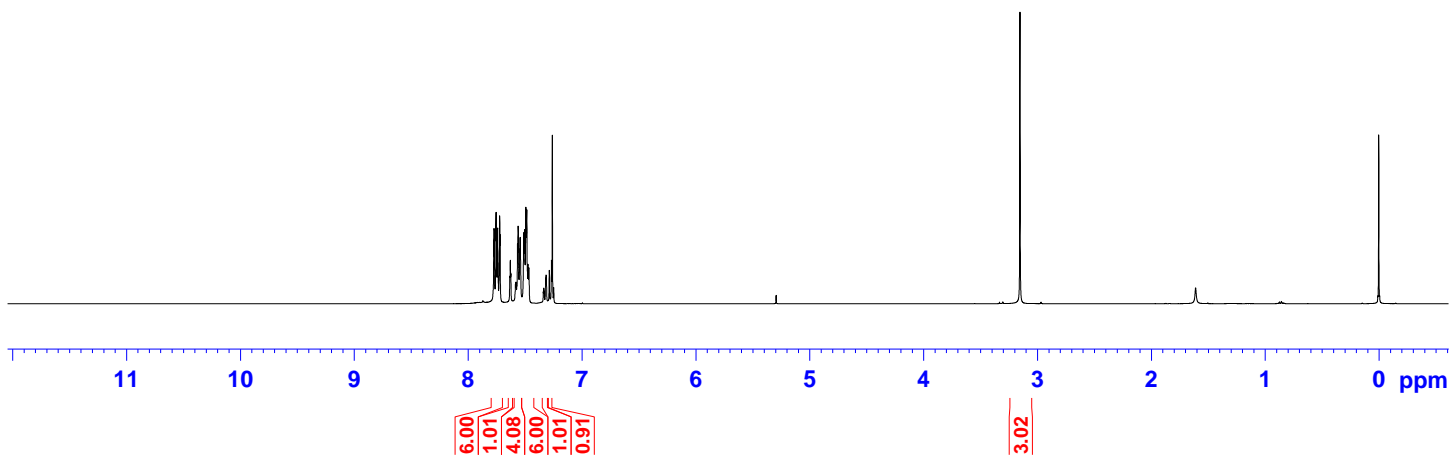

\section{${ }^{13} \mathrm{C}$ NMR (100 MHz, $\mathrm{CDCl}_{3}$ )}

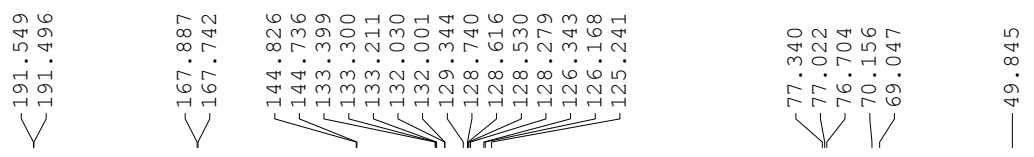<smiles>COC(=O)C(=P)C(=O)c1cccc(Cl)c1</smiles>

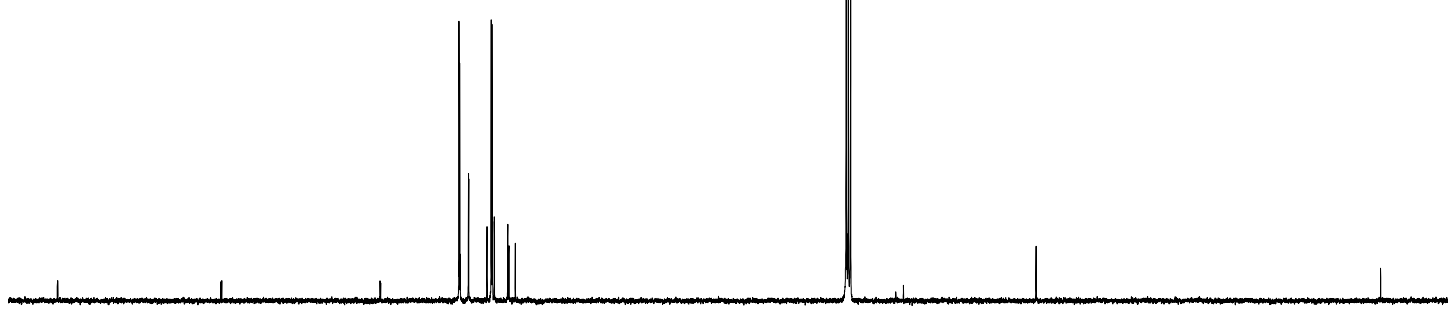

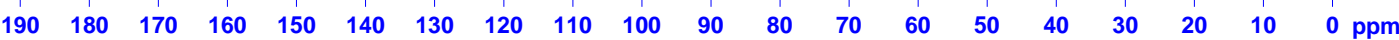


${ }^{31}$ P NMR (162 MHz, $\left.\mathrm{CDCl}_{3}\right)$<smiles>COC(=O)C(=Pc1ccccc1)C(=O)c1cccc(Cl)c1</smiles>

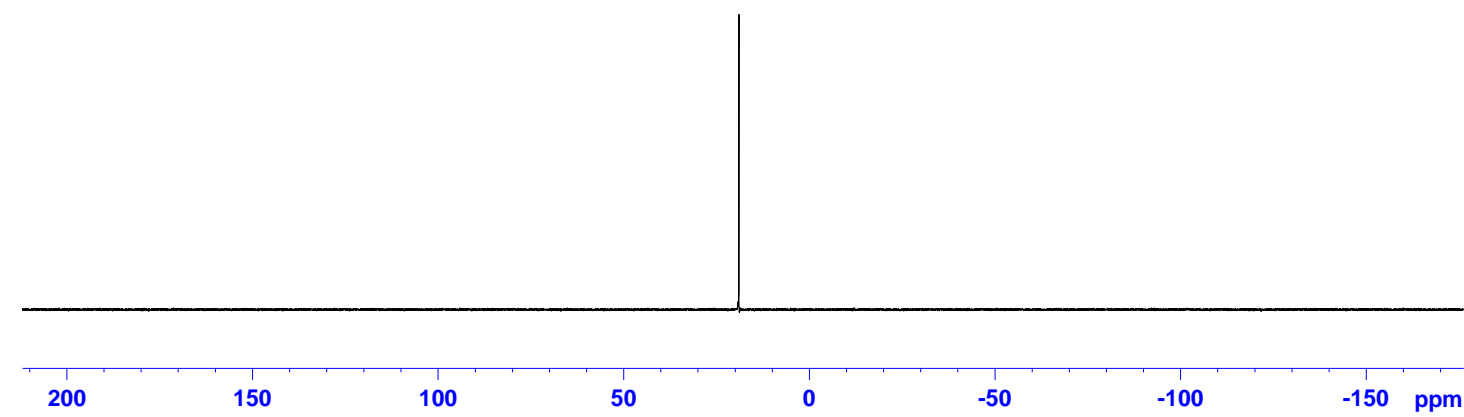

Methyl 3-(3-bromophenyl)-3-oxo-2-(triphenylphosphoranylidene)propanoate 18:

${ }^{1} \mathrm{H}$ NMR (400 MHz, $\left.\mathrm{CDCl}_{3}\right)$

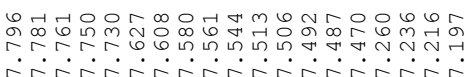

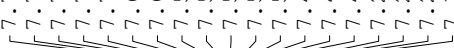<smiles>COC(=O)C(=Pc1ccccc1)C(=O)c1cccc(Br)c1</smiles>

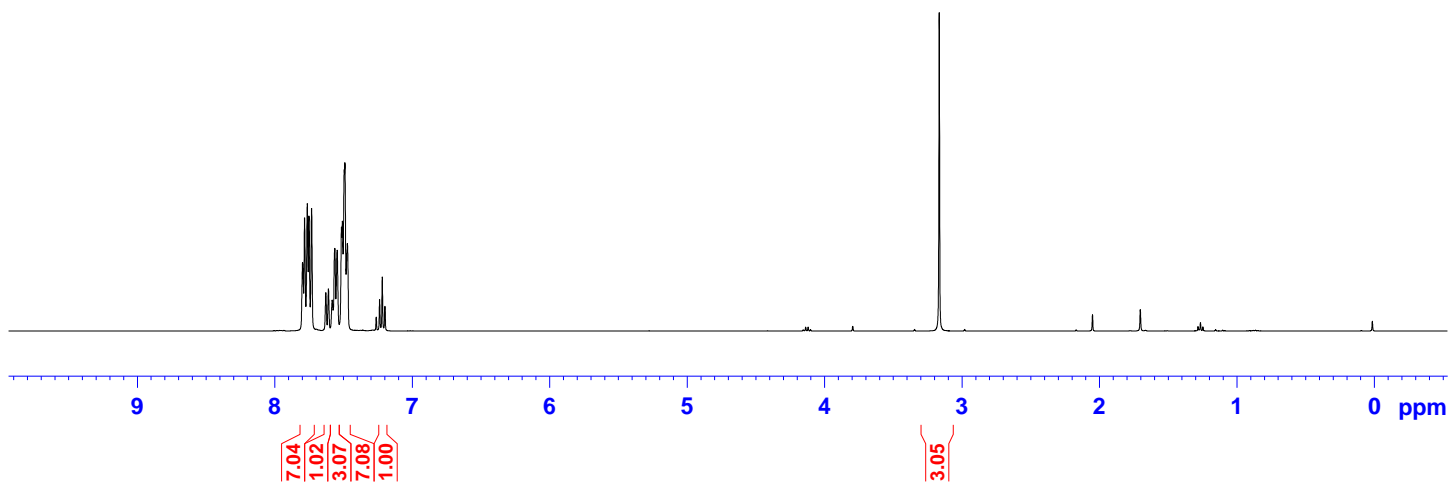




\section{${ }^{13}$ C NMR (100 MHz, $\left.\mathrm{CDCl}_{3}\right)$}

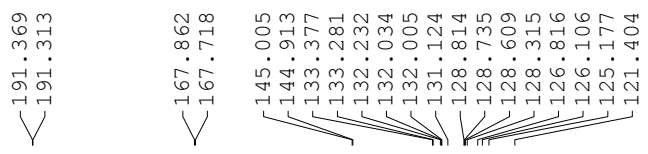<smiles>COC(=O)C(=P)C(=O)c1cccc(Br)c1</smiles>

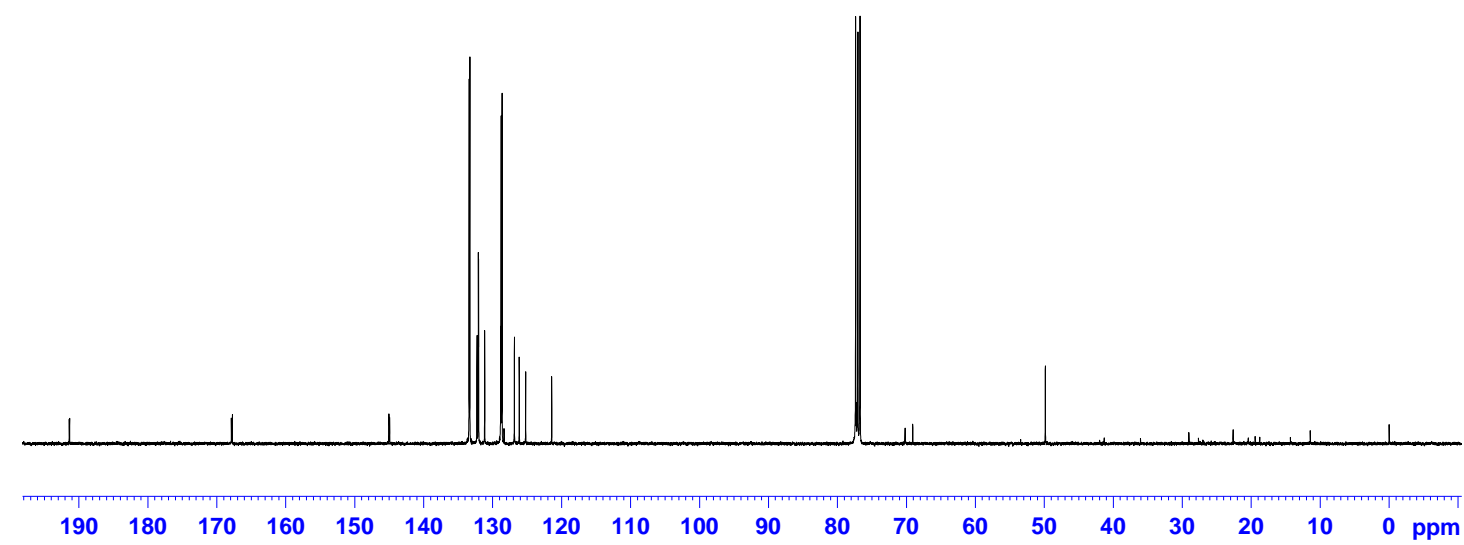

\section{${ }^{31}$ P NMR (162 MHz, $\left.\mathrm{CDCl}_{3}\right)$}<smiles>COC(=O)C(=P)C(=O)c1cccc(Br)c1</smiles>

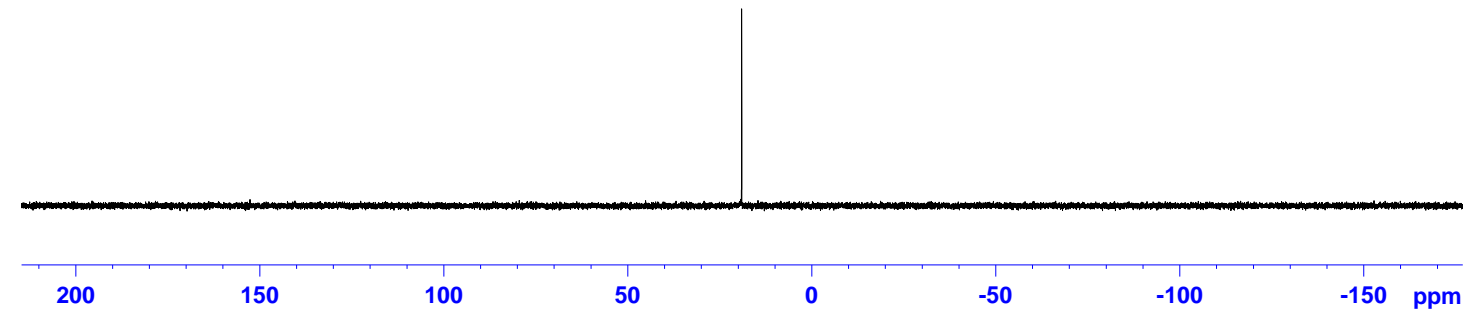


Methyl 3-(3-nitrophenyl)-3-oxo-2-(triphenylphosphoranylidene)propanoate 19:

${ }^{1}$ H NMR (400 MHz, CDCl 3$)$

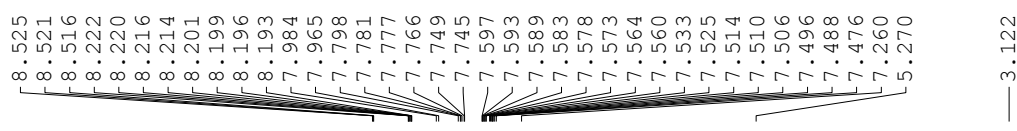<smiles>COC(=O)C(=P)C(=O)c1cccc([N+](=O)[O-])c1</smiles>

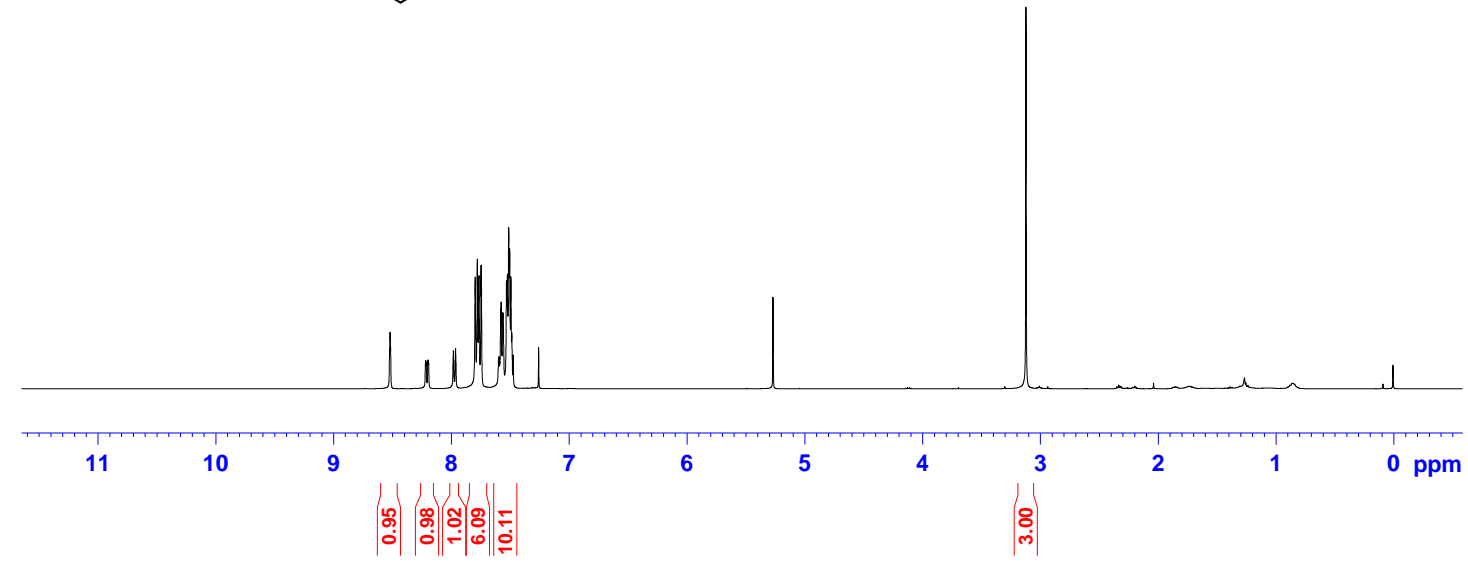

\section{${ }^{13} \mathrm{C}$ NMR (100 MHz, $\left.\mathrm{CDCl}_{3}\right)$}

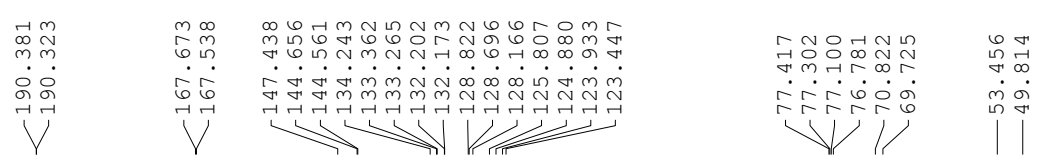<smiles>COC(=O)C(=P)C(=O)c1cccc([N+](=O)[O-])c1</smiles>

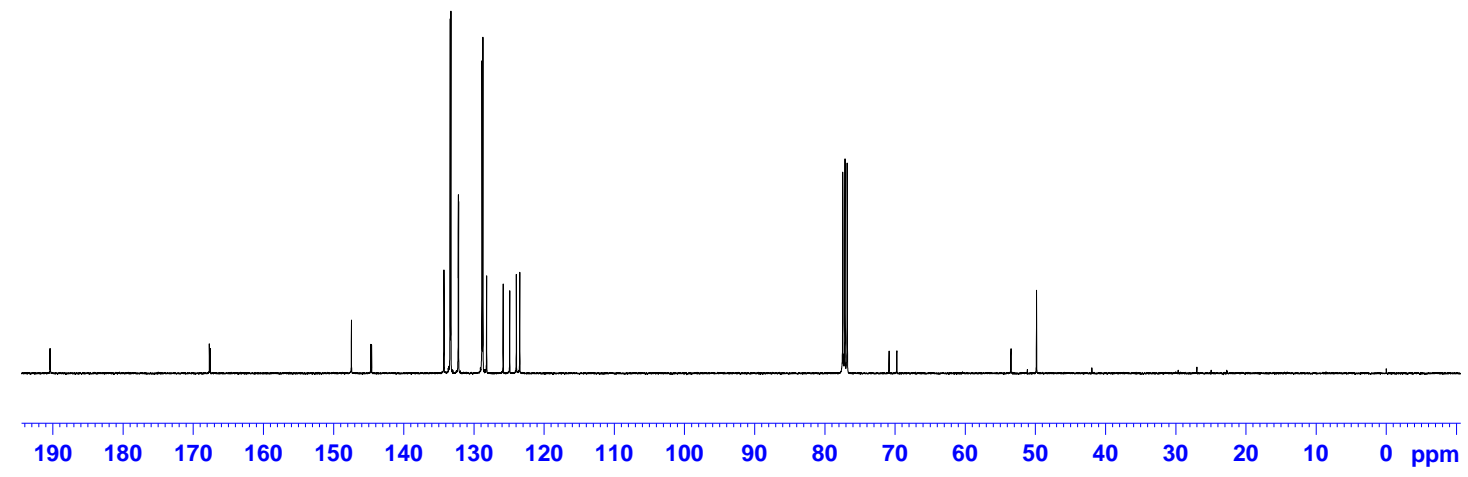


${ }^{31}$ P NMR (162 MHz, $\left.\mathrm{CDCl}_{3}\right)$<smiles>COC(=O)C(=Pc1ccccc1)C(=O)c1cccc([N+](=O)[O-])c1</smiles>

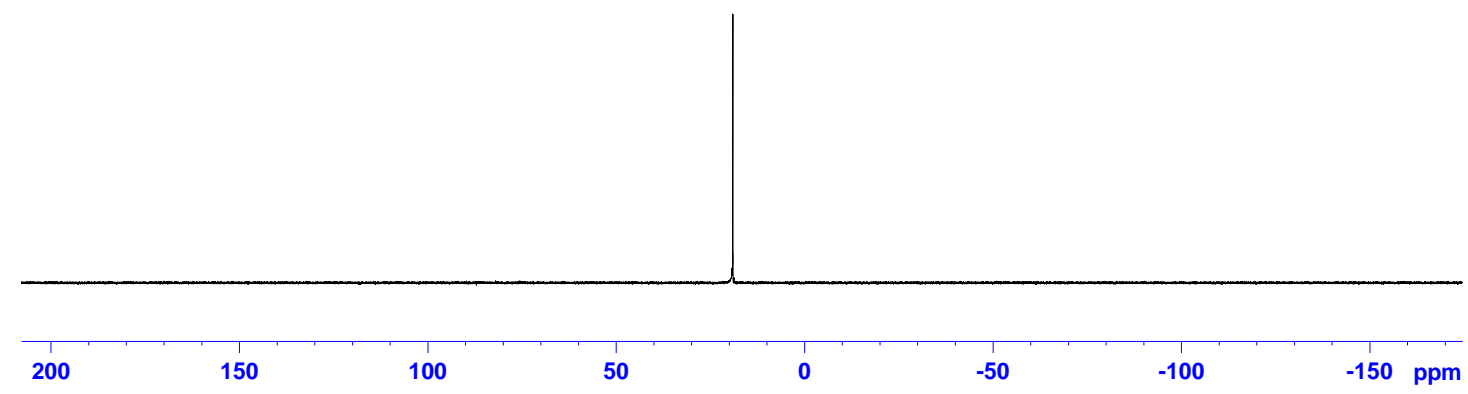

Methyl 3-oxo-3-(m-tolyl)-2-(triphenylphosphoranylidene)propanoate 20:

${ }^{1} \mathrm{H}$ NMR (400 MHz, $\left.\mathrm{CDCl}_{3}\right)$

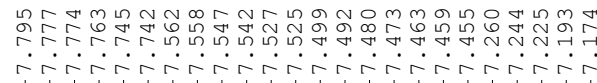

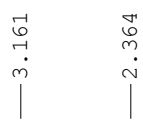<smiles>COC(=O)C(=Pc1ccccc1)C(=O)c1cccc(C)c1</smiles>

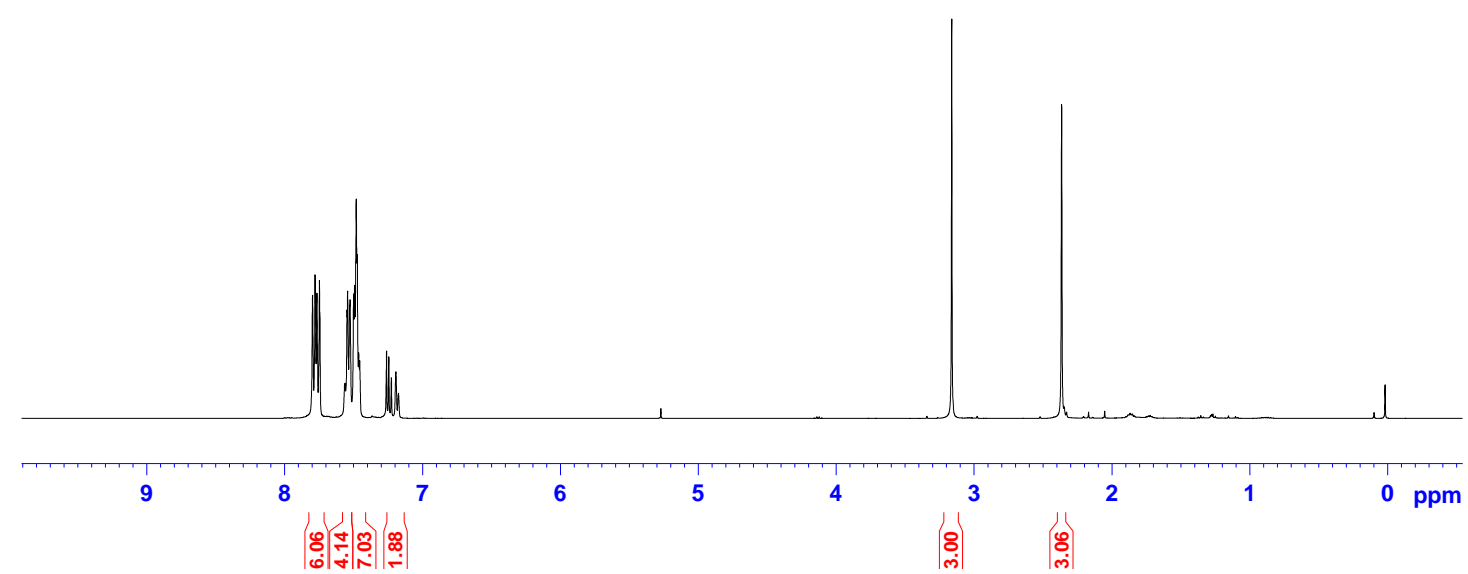




\section{${ }^{13}$ C NMR (100 MHz, $\left.\mathrm{CDCl}_{3}\right)$}
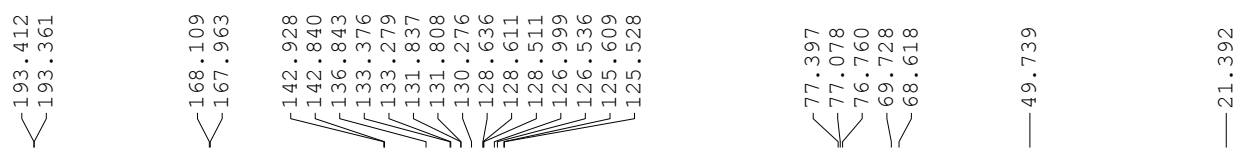<smiles>COC(=O)C(=PP(c1ccccc1)c1ccccc1)C(=O)c1cccc(C)c1</smiles>

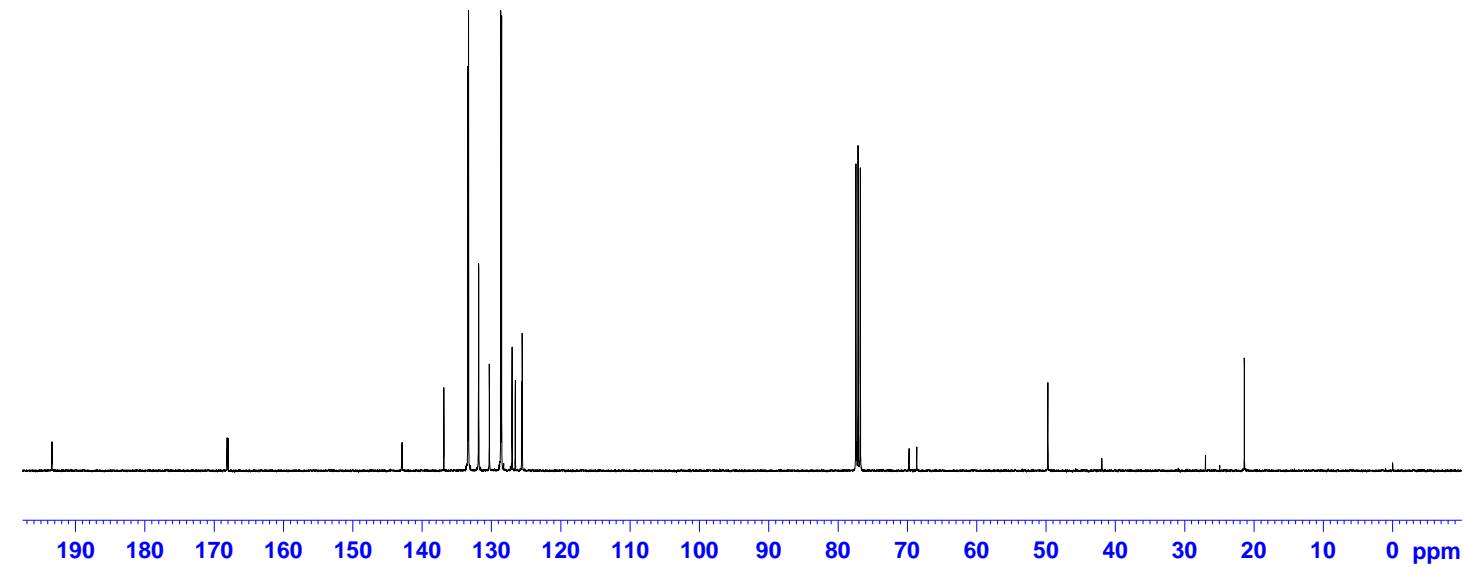

${ }^{31}$ P NMR (162 MHz, $\mathrm{CDCl}_{3}$ )<smiles>COC(=O)C(=Pc1ccccc1)C(=O)c1cccc(C)c1</smiles>

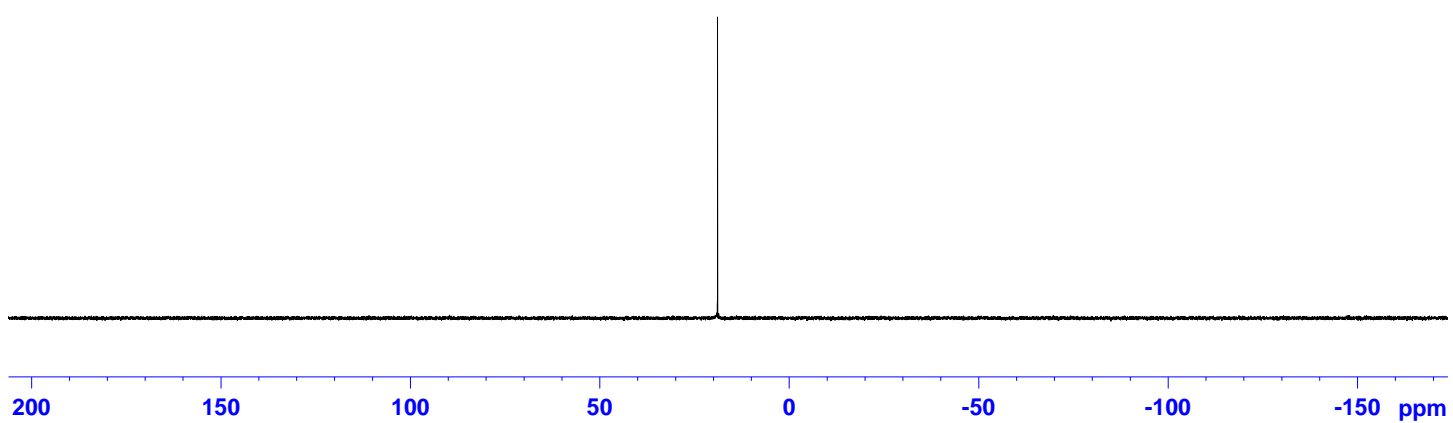


Methyl-3-(3-methoxyphenyl)-3-oxo-2-(triphenylphosphoranylidene)propanoate 21: ${ }^{1} \mathrm{H}$ NMR (400 MHz, $\left.\mathrm{CDCl}_{3}\right)$

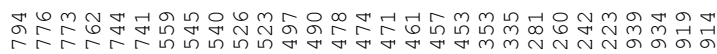

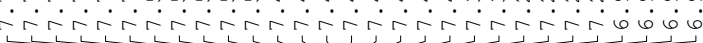<smiles>COC(=O)C(=P)C(=O)c1cccc(OC)c1</smiles>

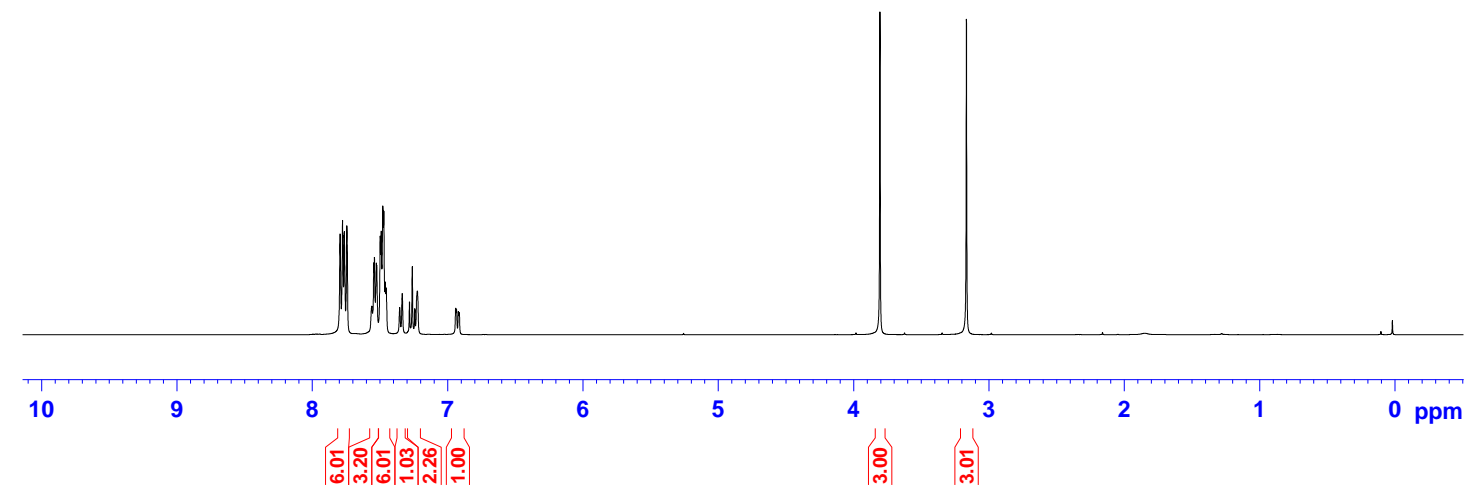

${ }^{13} \mathrm{C}$ NMR (100 MHz, $\left.\mathrm{CDCl}_{3}\right)$

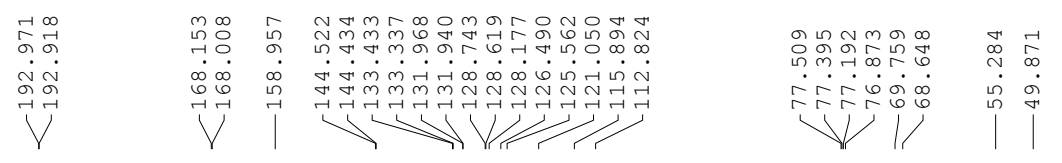<smiles>COC(=O)C(=P)C(=O)c1cccc(OC)c1</smiles>

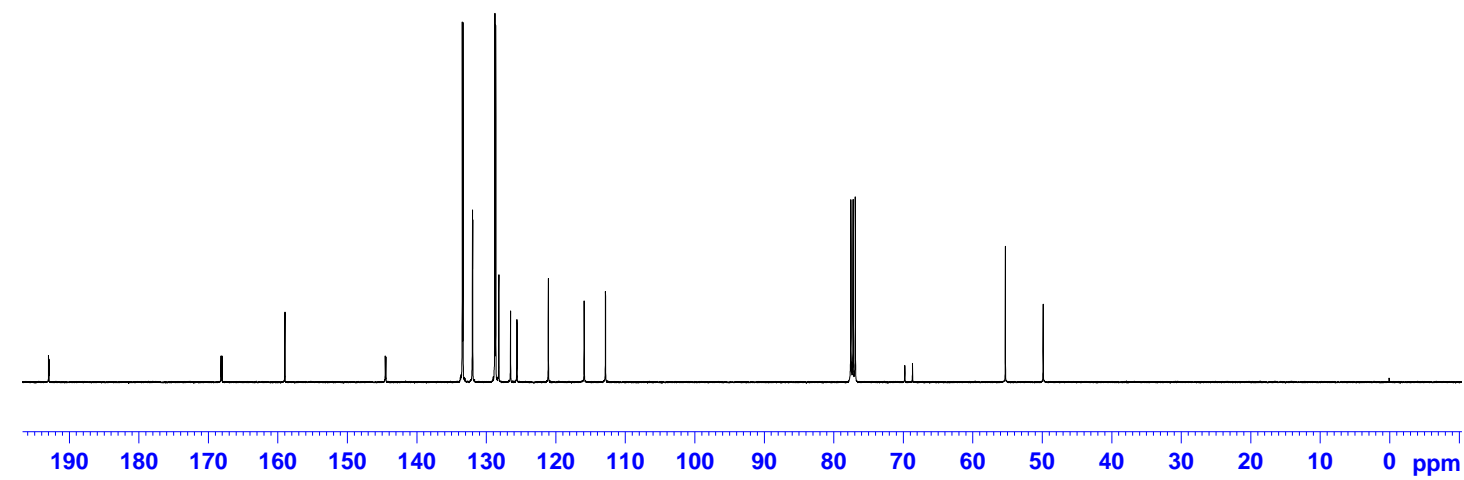


${ }^{31} \mathrm{P}$ NMR (162 MHz, $\left.\mathrm{CDCl}_{3}\right)$<smiles>COC(=O)C(Pc1ccccc1)C(=O)c1cccc(OC)c1</smiles>

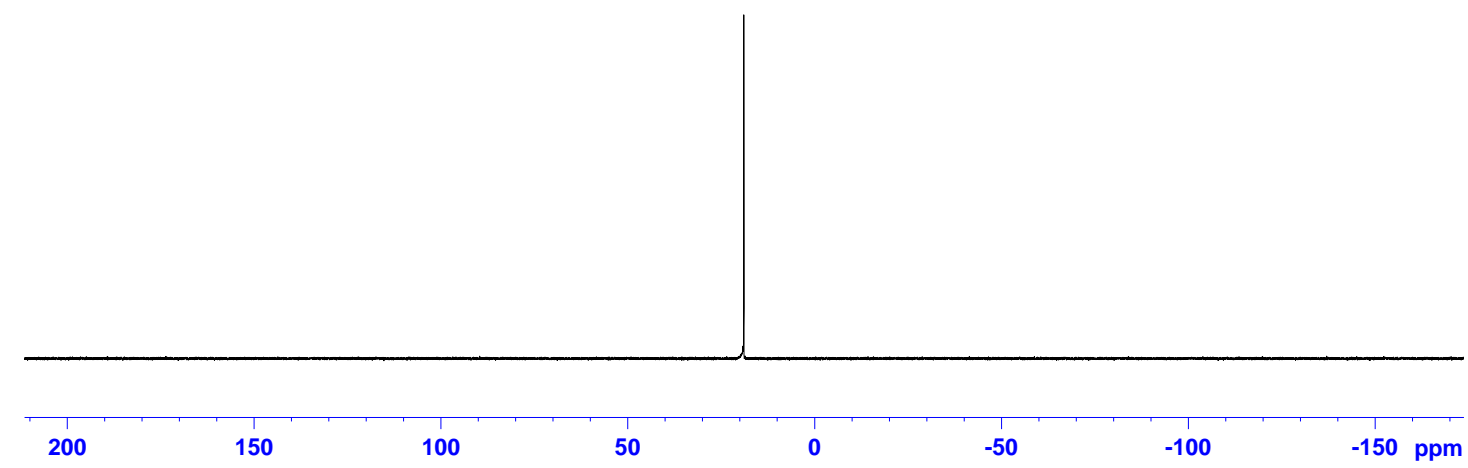

Methyl 2-(3-methoxy-3-oxo-2-(triphenylphosphoranylidene)propanoyl)benzoate 22: ${ }^{1} \mathrm{H}$ NMR (400 MHz, $\left.\mathrm{CDCl}_{3}\right)$

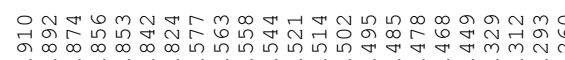

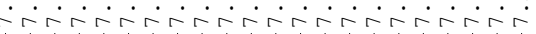

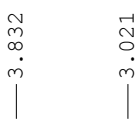<smiles>COC(=O)c1ccccc1C(=O)C(=P)C(=O)c1ccccc1</smiles>

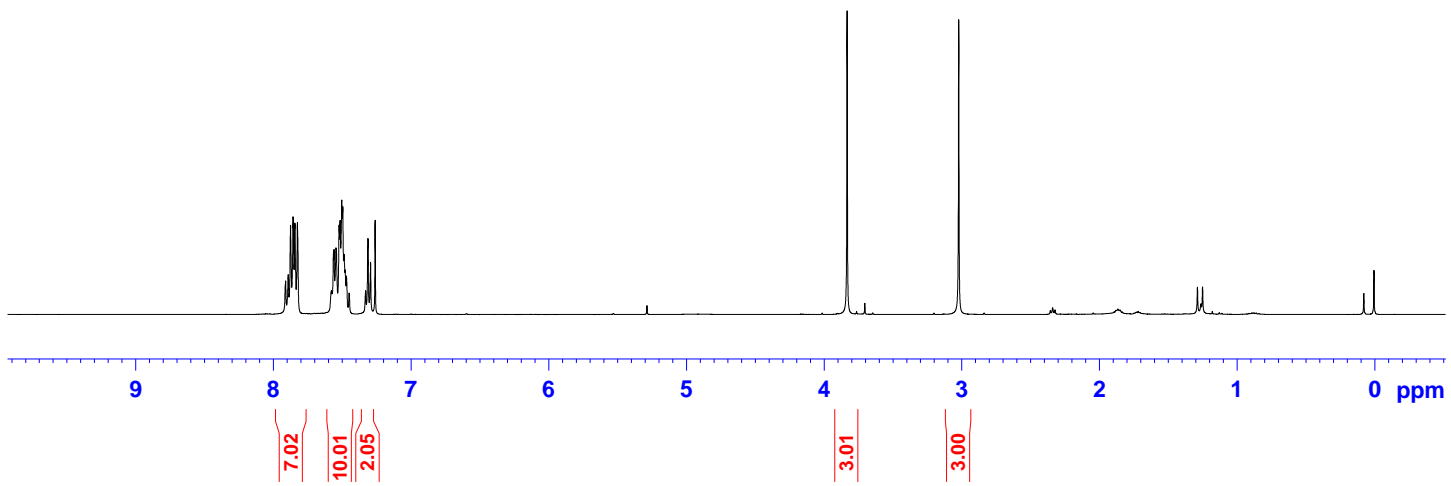




\section{${ }^{13}$ C NMR (100 MHz, $\left.\mathrm{CDCl}_{3}\right)$}

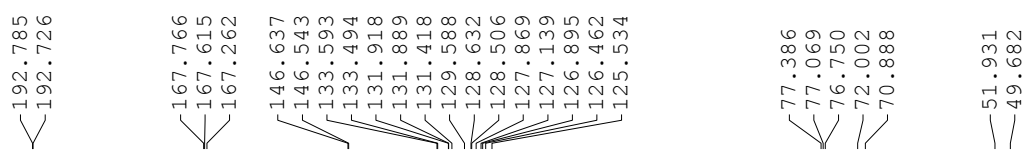<smiles>COC(=O)C(=P)C(=P)C(=O)c1ccccc1C(=O)OC</smiles>

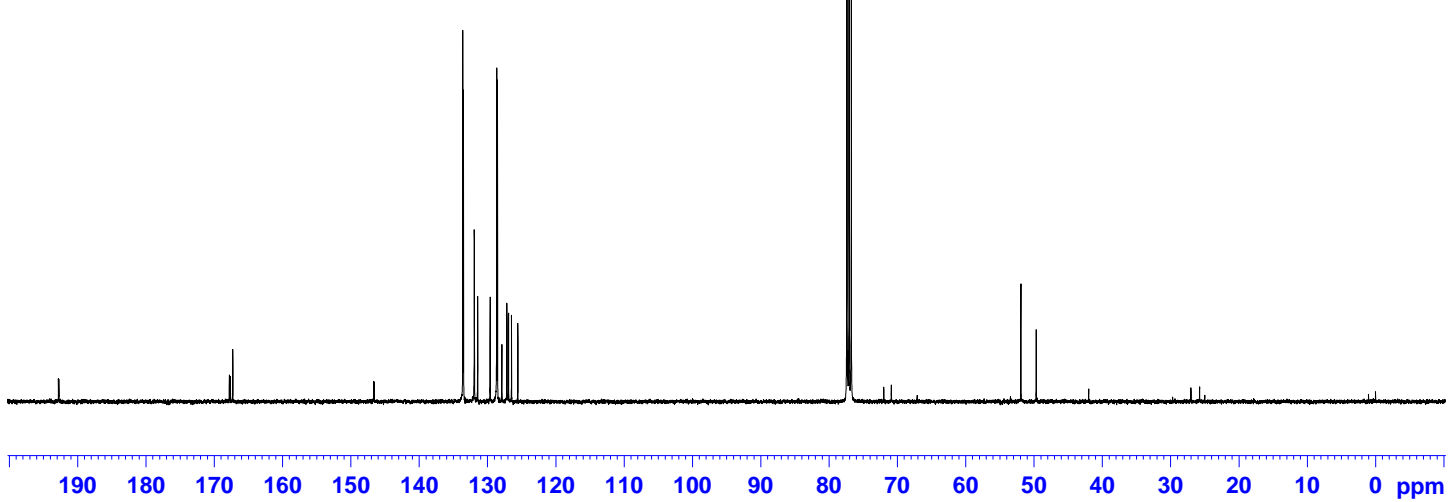

${ }^{31}$ P NMR (162 MHz, $\left.\mathrm{CDCl}_{3}\right)$<smiles>COC(=O)c1ccccc1C(=O)C(=P)C(=O)c1ccccc1</smiles>

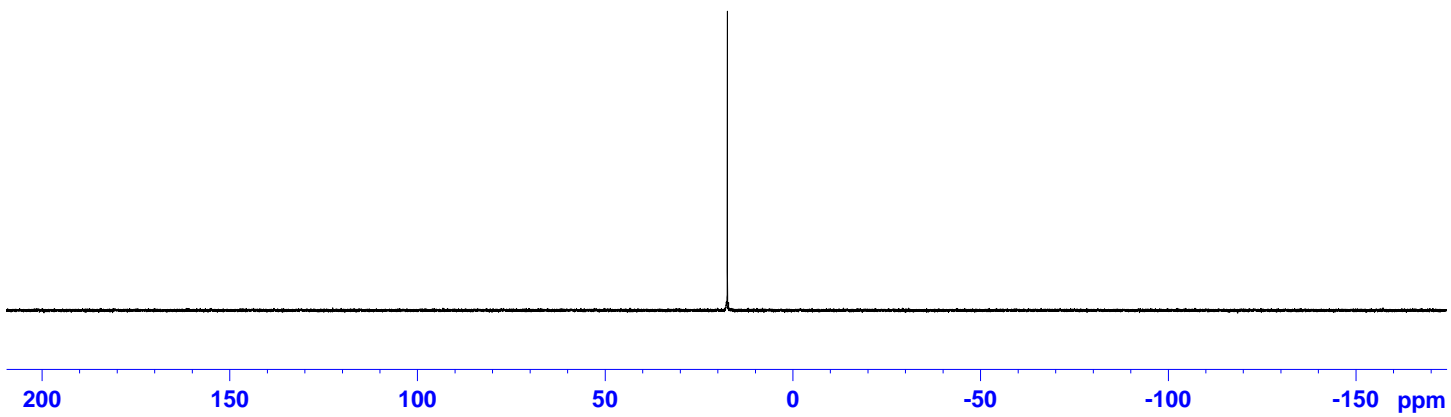


Methyl 3-(2-fluorophenyl)-3-oxo-2-(triphenylphosphoranylidene)propanoate 23:

${ }^{1}$ H NMR (400 MHz, $\left.\mathrm{CDCl}_{3}\right)$

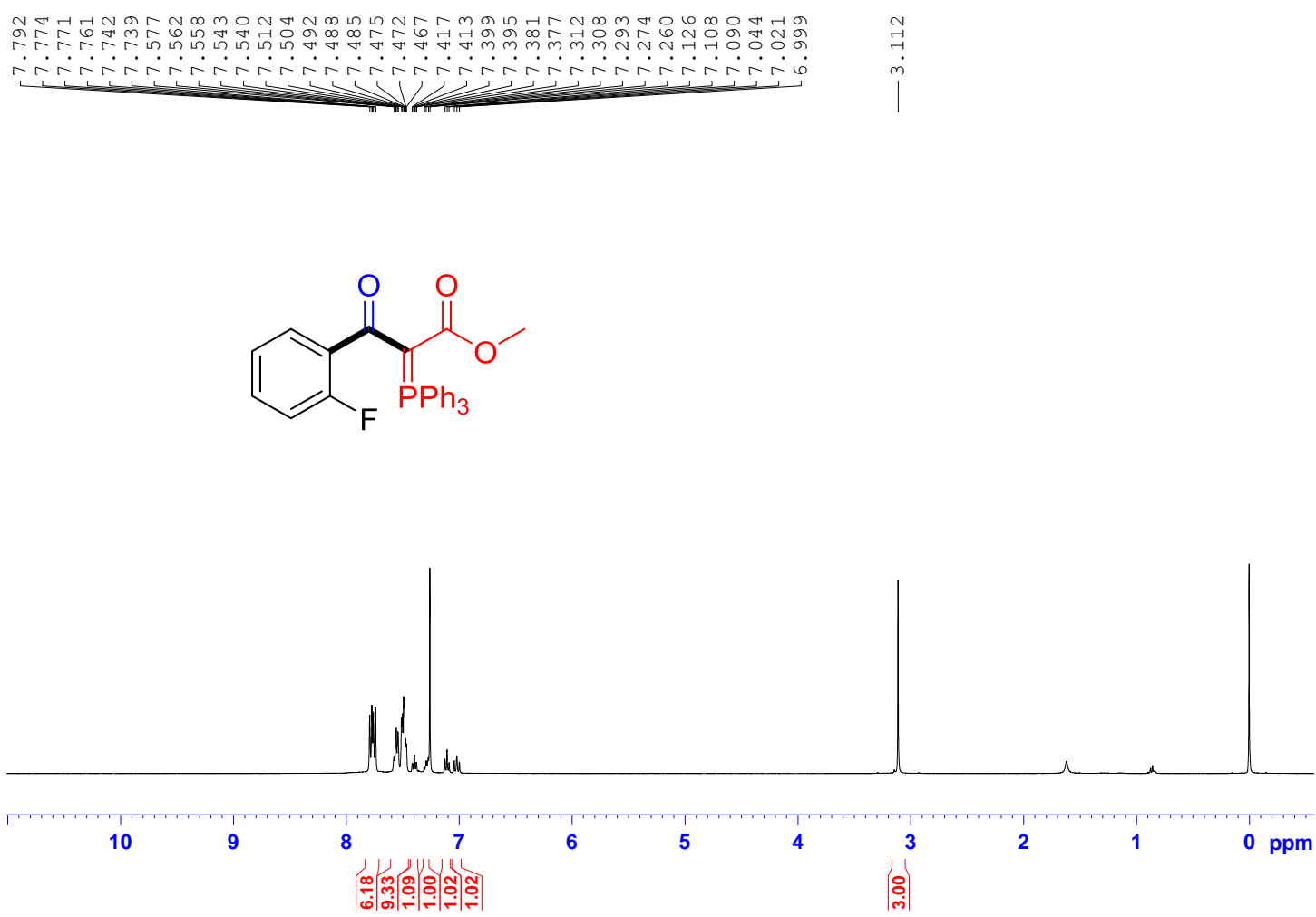

${ }^{13} \mathrm{C}$ NMR (100 MHz, $\left.\mathrm{CDCl}_{3}\right)$

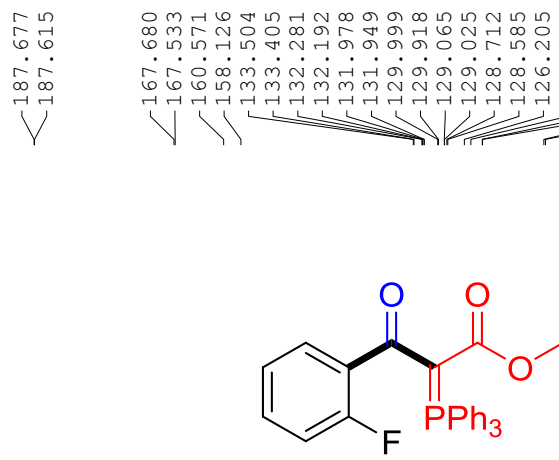

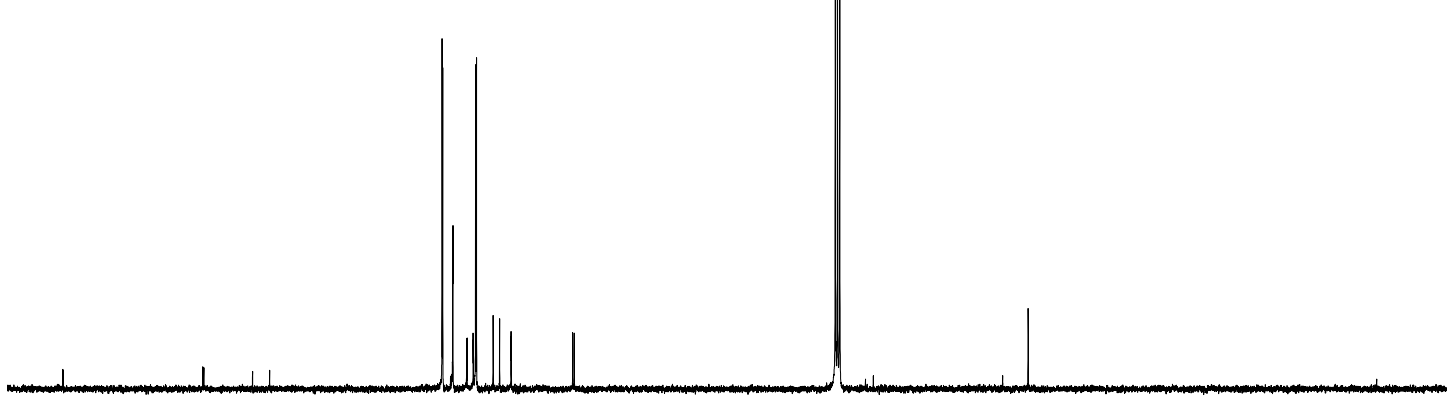

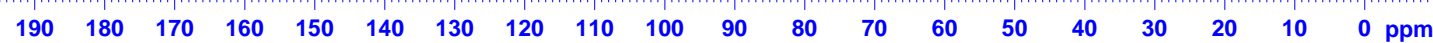


${ }^{31}$ P NMR (162 MHz, $\left.\mathrm{CDCl}_{3}\right)$<smiles>COC(=O)C(=P)C(=O)C(=O)c1ccccc1F</smiles>

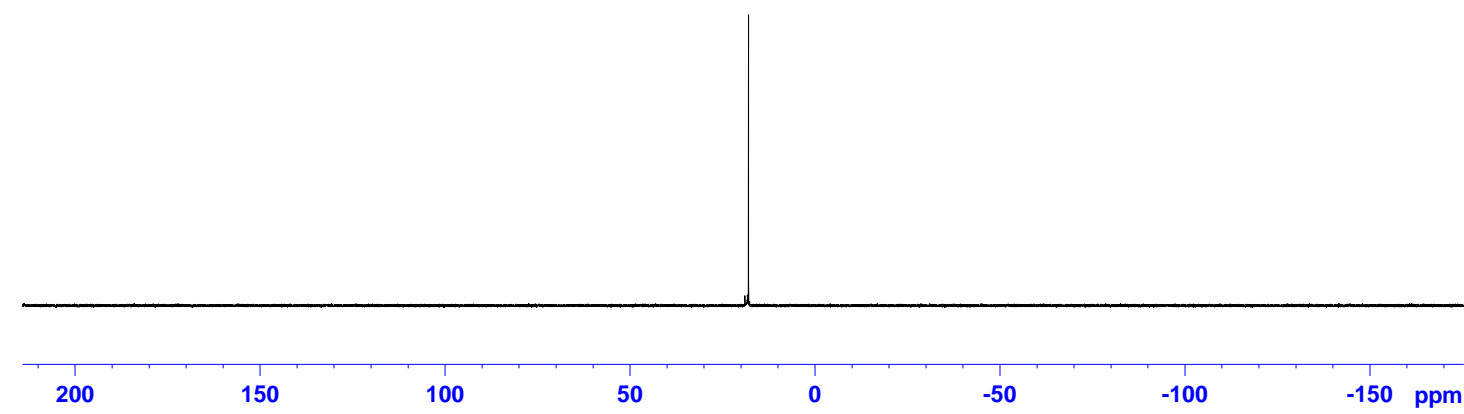

Methyl 3-(2-chlorophenyl)-3-oxo-2-(triphenylphosphoranylidene)propanoate 24:

${ }^{1}$ H NMR (400 MHz, $\left.\mathrm{CDCl}_{3}\right)$<smiles>COC(=O)C(=P)C(=O)c1ccccc1Cl</smiles>

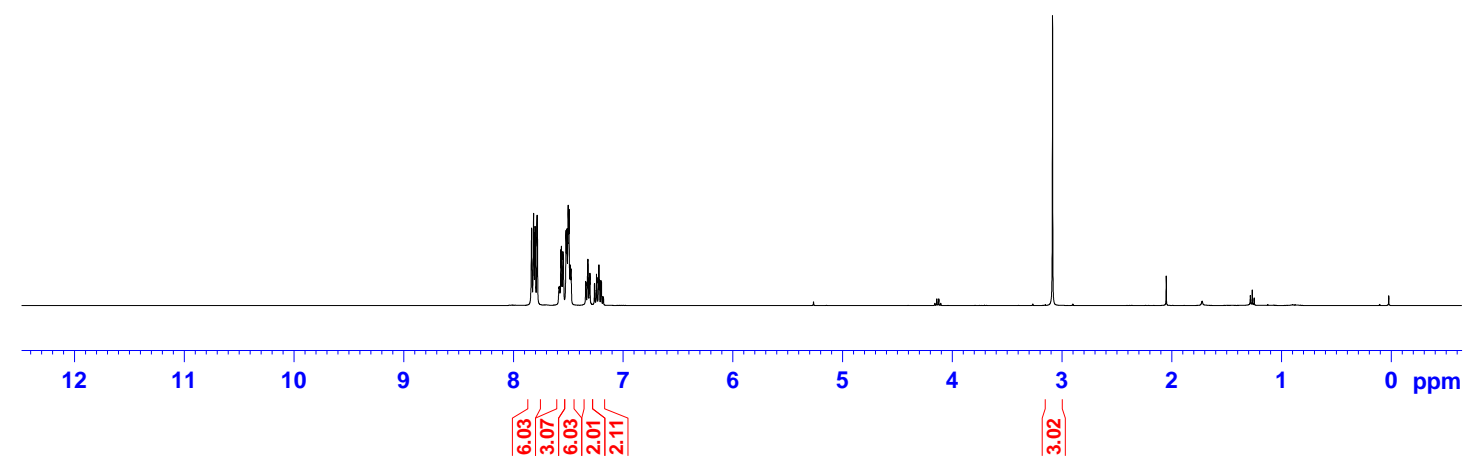




\section{${ }^{13} \mathrm{C}$ NMR (100 MHz, $\left.\mathrm{CDCl}_{3}\right)$}

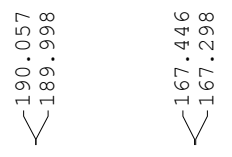

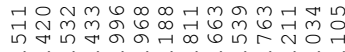

舟

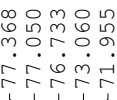

il

$\overbrace{\mathrm{Cl}_{P h_{3}}}^{O}$

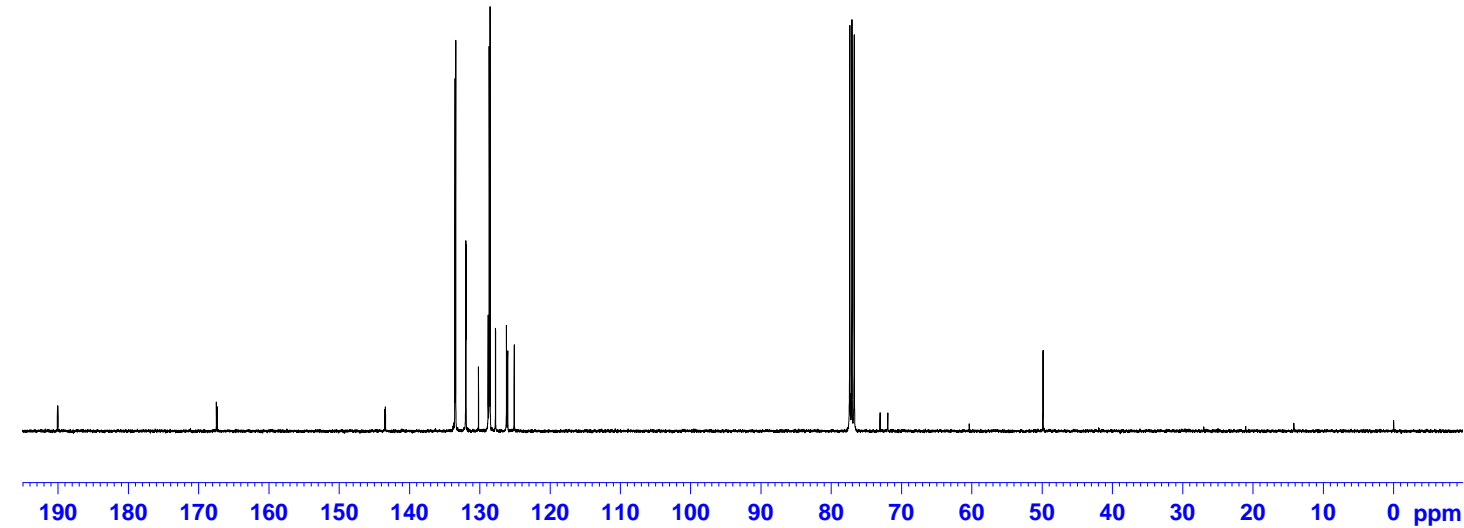

${ }^{31}$ P NMR (162 MHz, $\left.\mathrm{CDCl}_{3}\right)$<smiles>COC(=O)C(=P)C(=O)c1ccccc1Cl</smiles>

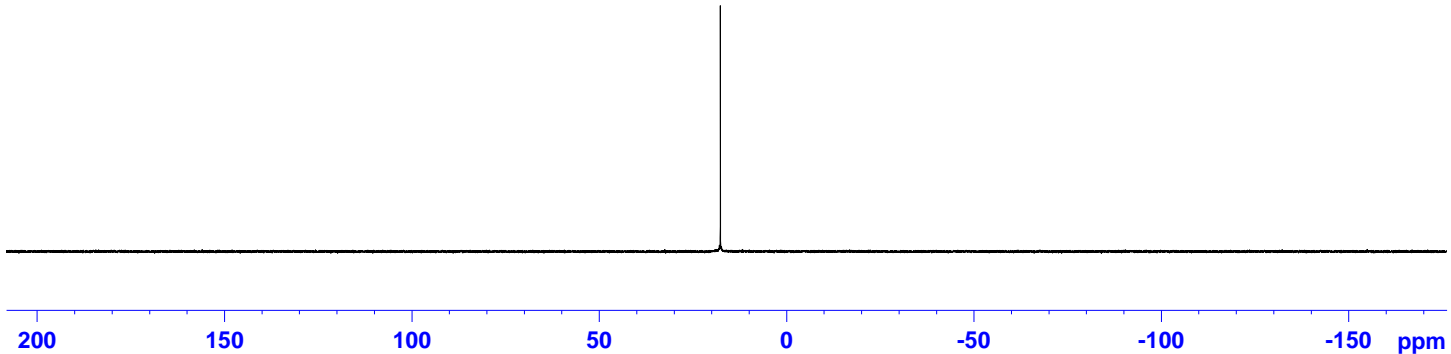


Methyl 3-(2-bromophenyl)-3-oxo-2-(triphenylphosphoranylidene)propanoate 25:

${ }^{1}$ H NMR (400 MHz, CDCl 3 )

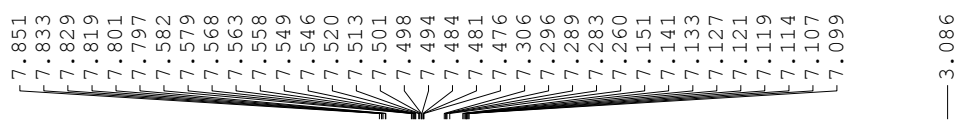<smiles>COC(=O)C(=Cc1ccccc1)C(=O)c1ccccc1Br</smiles>

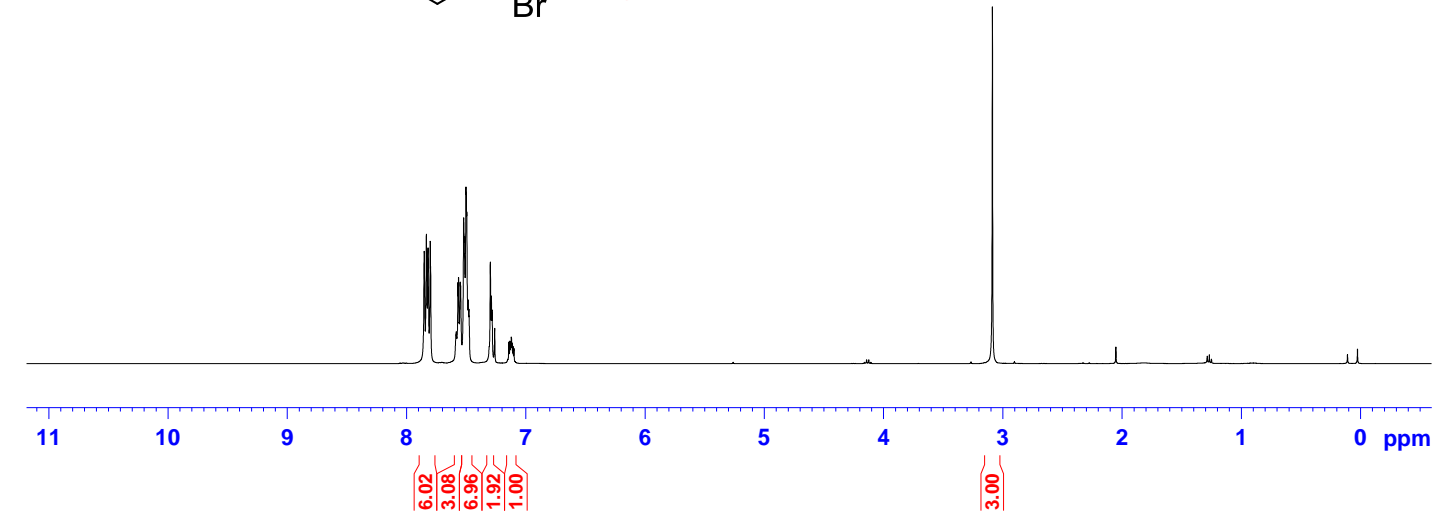

${ }^{13} \mathrm{C}$ NMR (100 MHz, $\mathrm{CDCl}_{3}$ )
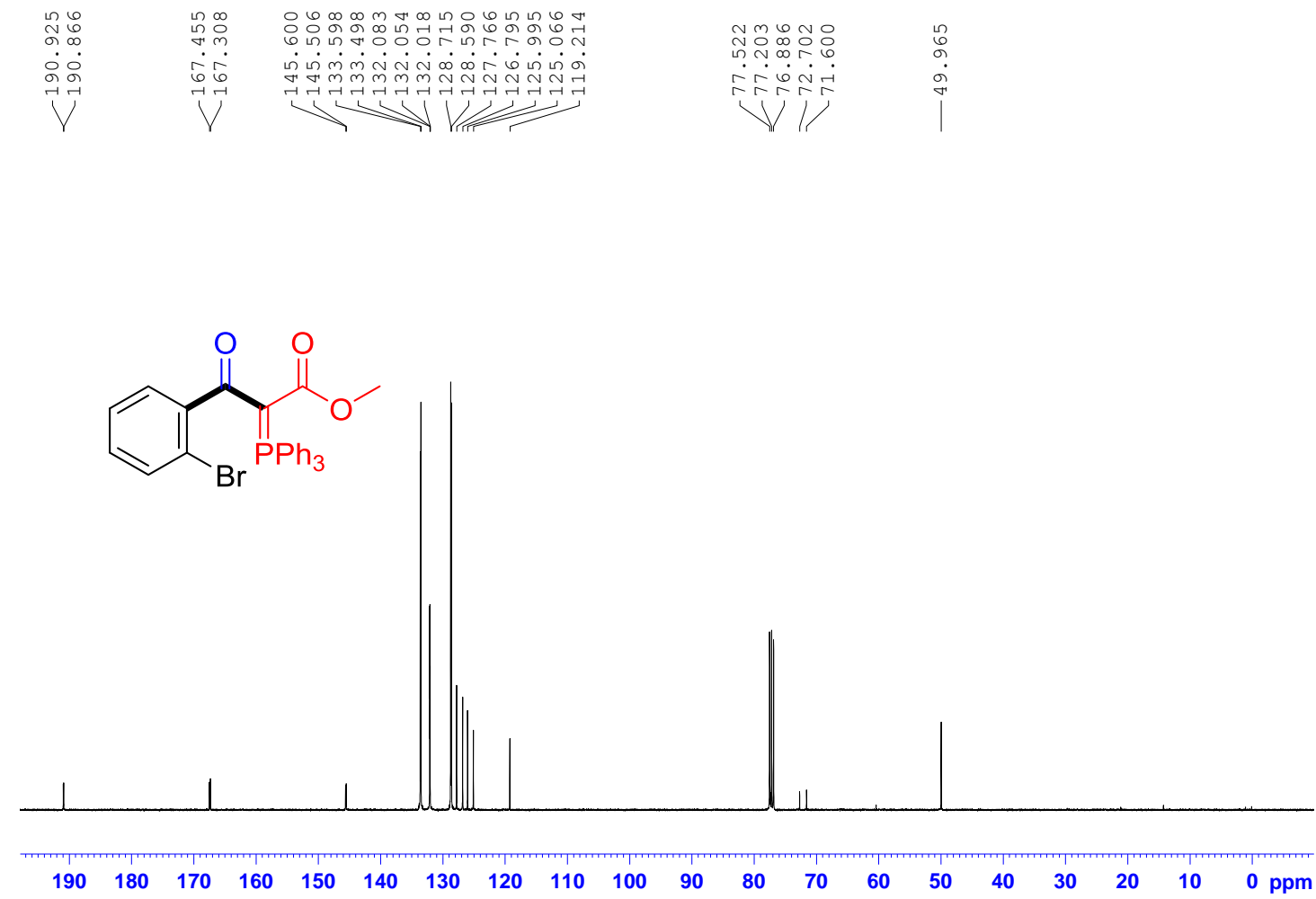
${ }^{31}$ P NMR (162 MHz, $\left.\mathrm{CDCl}_{3}\right)$<smiles>COC(=O)C(C(=O)c1ccccc1Br)=C(P)[PH](=O)c1ccccc1</smiles>

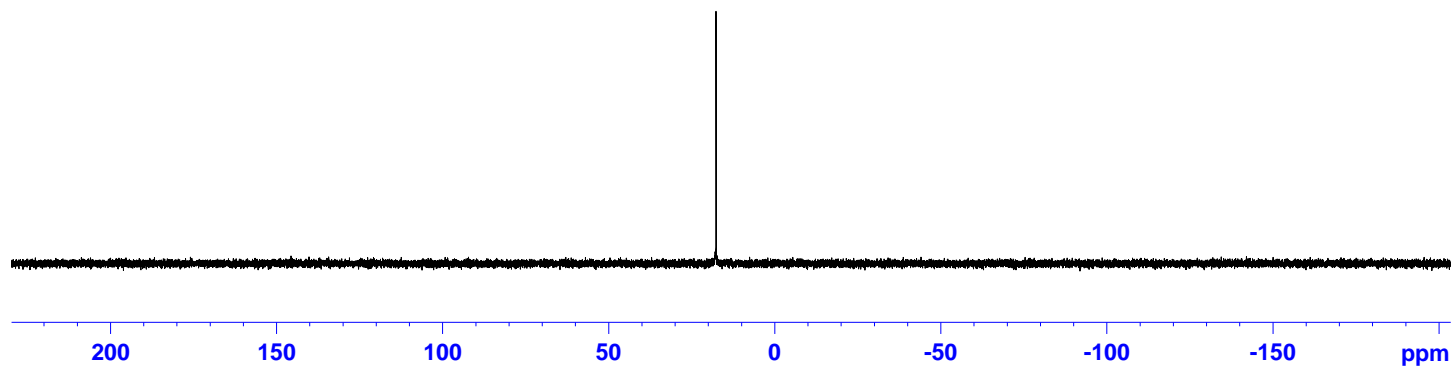

Methyl-3-oxo-3-(2-(trifluoromethoxy)phenyl)-2-(triphenylphosphoranylidene)propanoate 26: ${ }^{1} \mathrm{H}$ NMR (400 $\left.\mathrm{MHz}, \mathrm{CDCl}_{3}\right)$

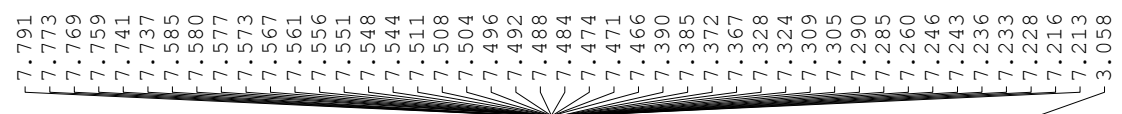<smiles>COC(=O)C(P)=C(C(=O)OC)C(=O)c1ccccc1OC(F)(F)F</smiles>

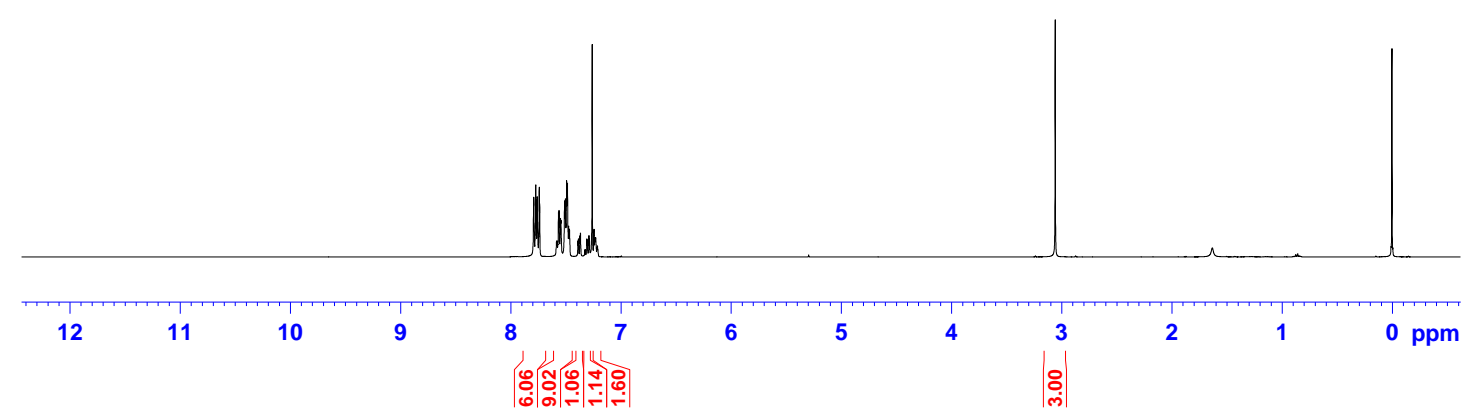




\section{${ }^{13} \mathrm{C}$ NMR (100 MHz, $\left.\mathrm{CDCl}_{3}\right)$}
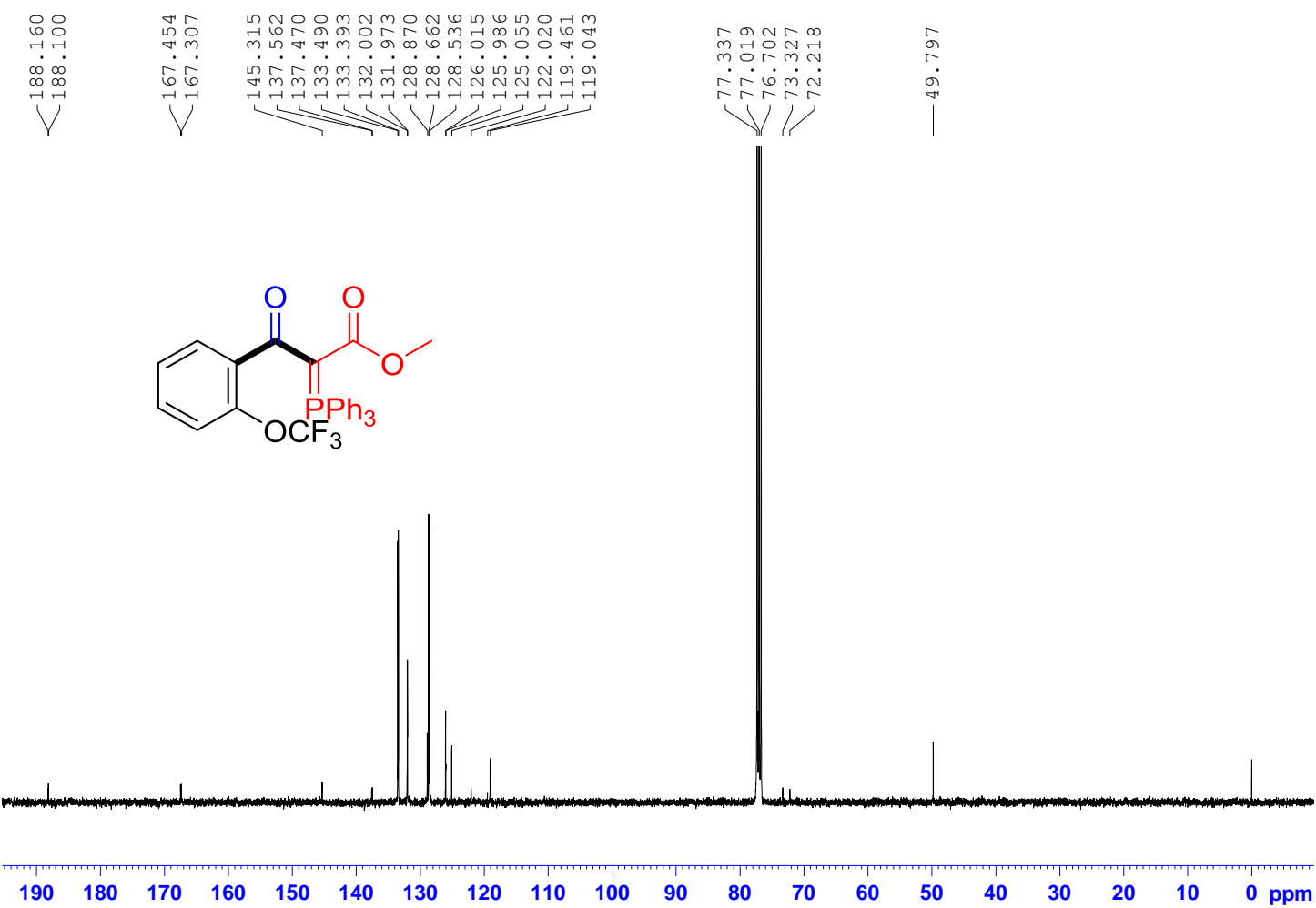

${ }^{31}$ P NMR (162 MHz, $\mathrm{CDCl}_{3}$ )<smiles>COC(=O)C(C(=O)c1ccccc1OC(F)(F)F)=C(C)C</smiles>

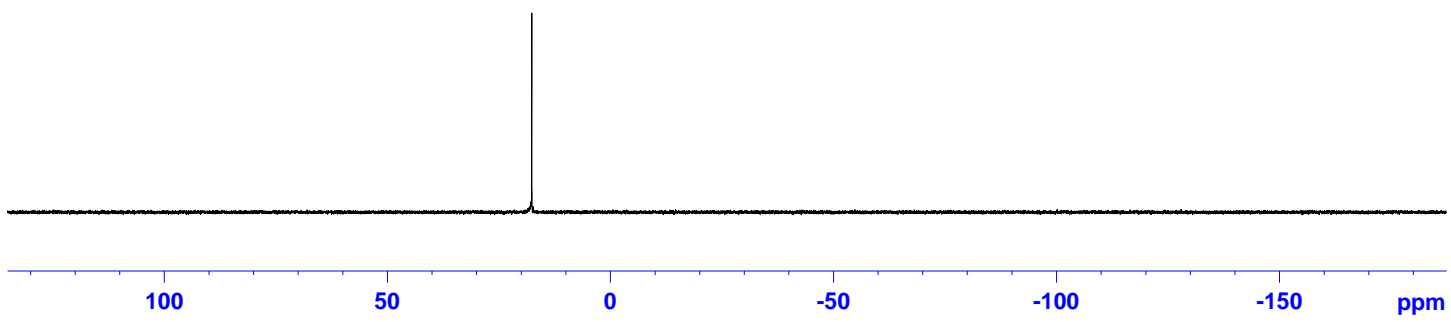


Methyl 3-oxo-3-(o-tolyl)-2-(triphenylphosphoranylidene)propanoate 27:

${ }^{1} \mathrm{H}$ NMR (400 MHz, $\left.\mathrm{CDCl}_{3}\right)$

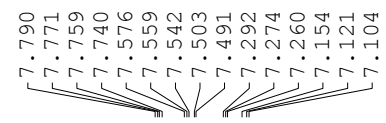<smiles>COC(=O)C(=P)C(=P)C(=O)c1ccccc1C</smiles>

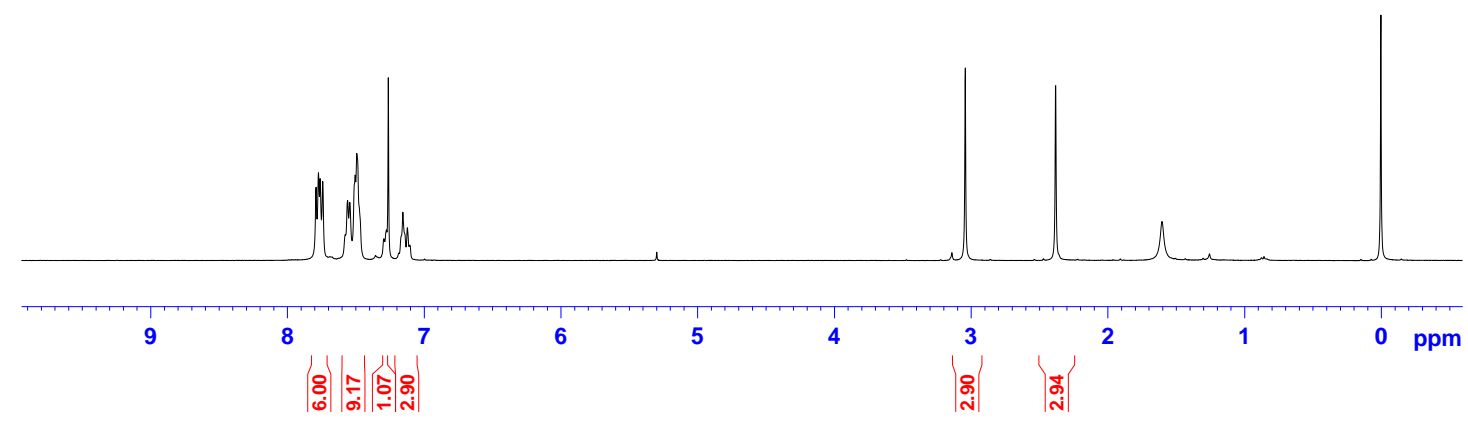

\section{${ }^{13} \mathrm{C}$ NMR (100 MHz, $\left.\mathrm{CDCl}_{3}\right)$}

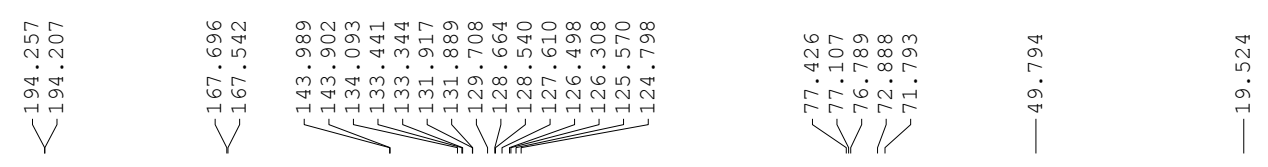<smiles>COC(=O)C(=P)C(=O)c1ccccc1C</smiles>

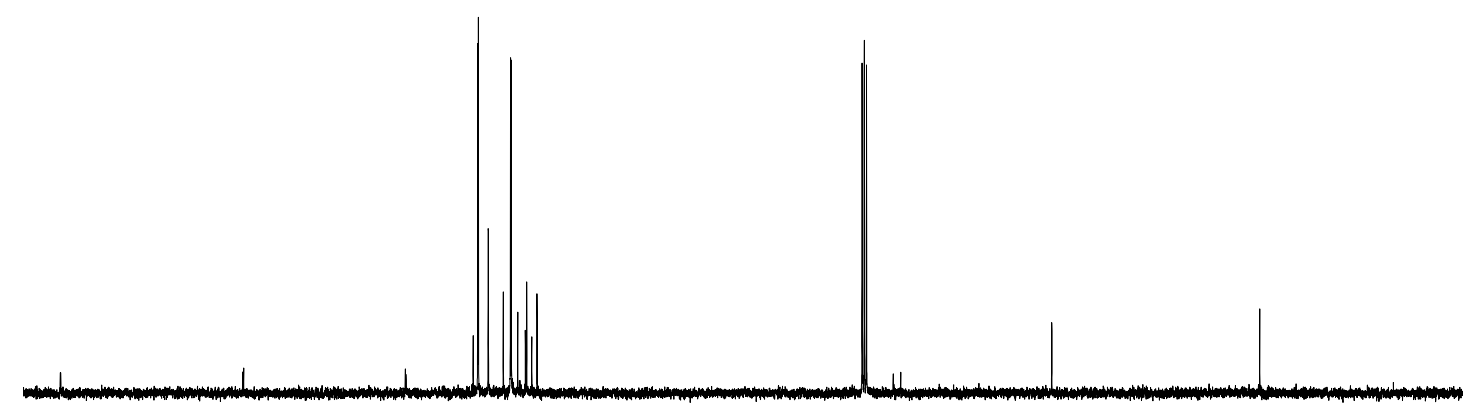

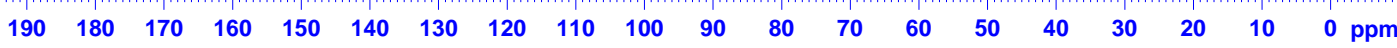


${ }^{31}$ P NMR (162 MHz, $\left.\mathrm{CDCl}_{3}\right)$<smiles>COC(=O)C(=P)C(=O)c1ccccc1C</smiles>

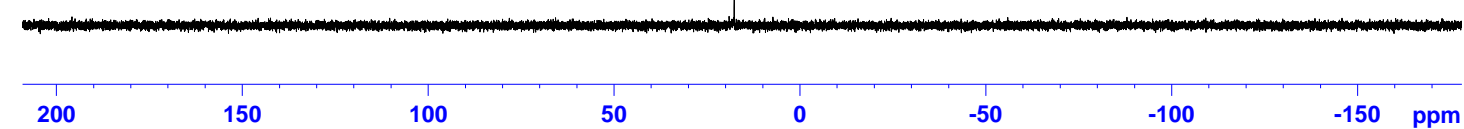

Methyl 3-(2-methoxyphenyl)-3-oxo-2-(triphenylphosphoranylidene)propanoate 28: ${ }^{1} \mathrm{H}$ NMR (400 $\left.\mathrm{MHz}, \mathrm{CDCl}_{3}\right)$

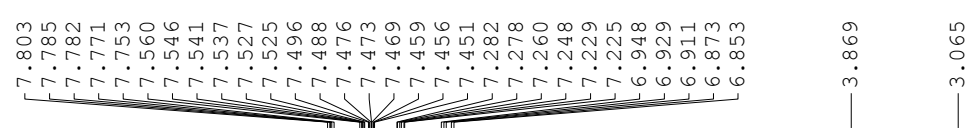

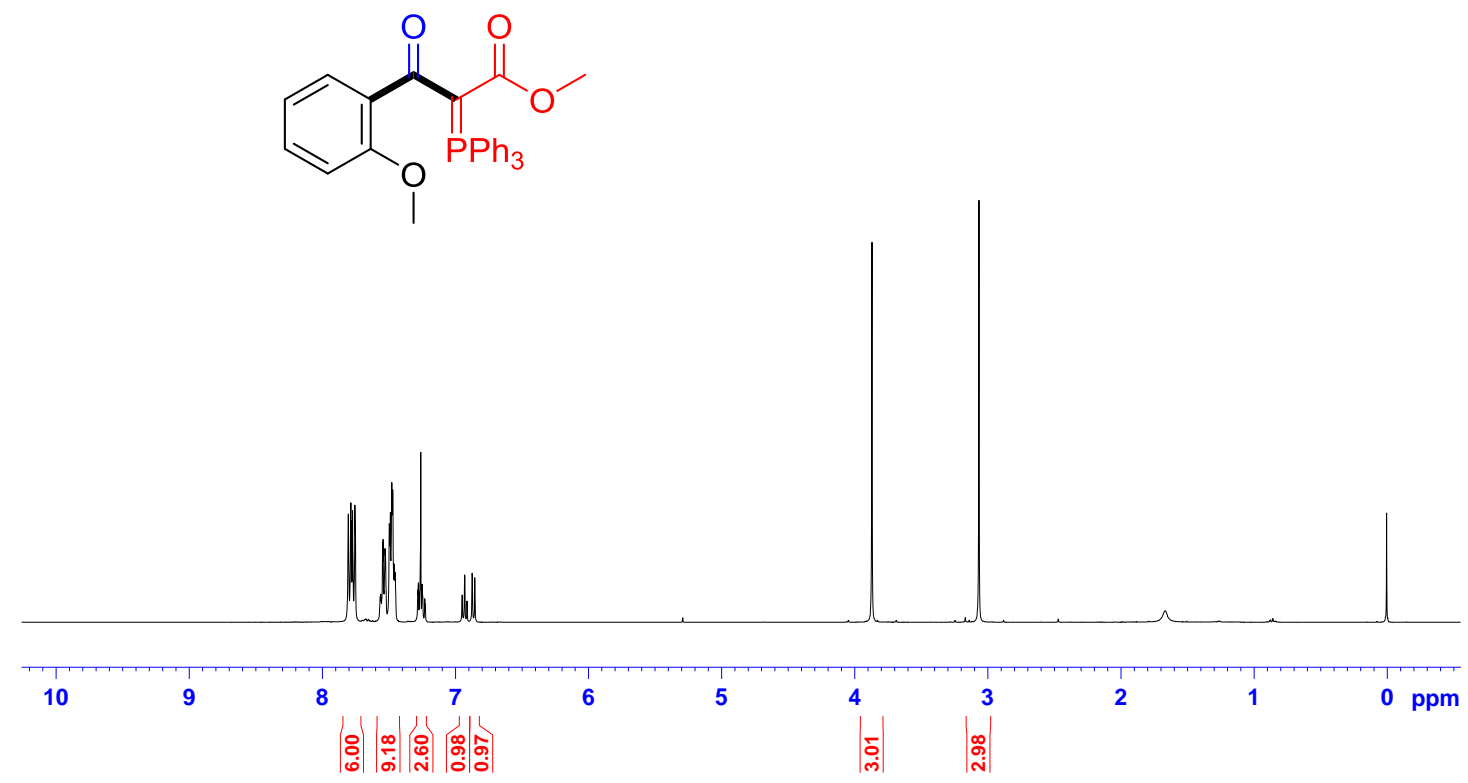


${ }^{13} \mathrm{C}$ NMR (100 MHz, $\mathrm{CDCl}_{3}$ )

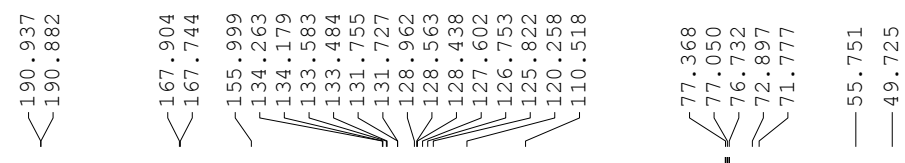<smiles>COC(=O)C(PC(=O)c1ccccc1)C(=O)c1ccccc1OC</smiles>

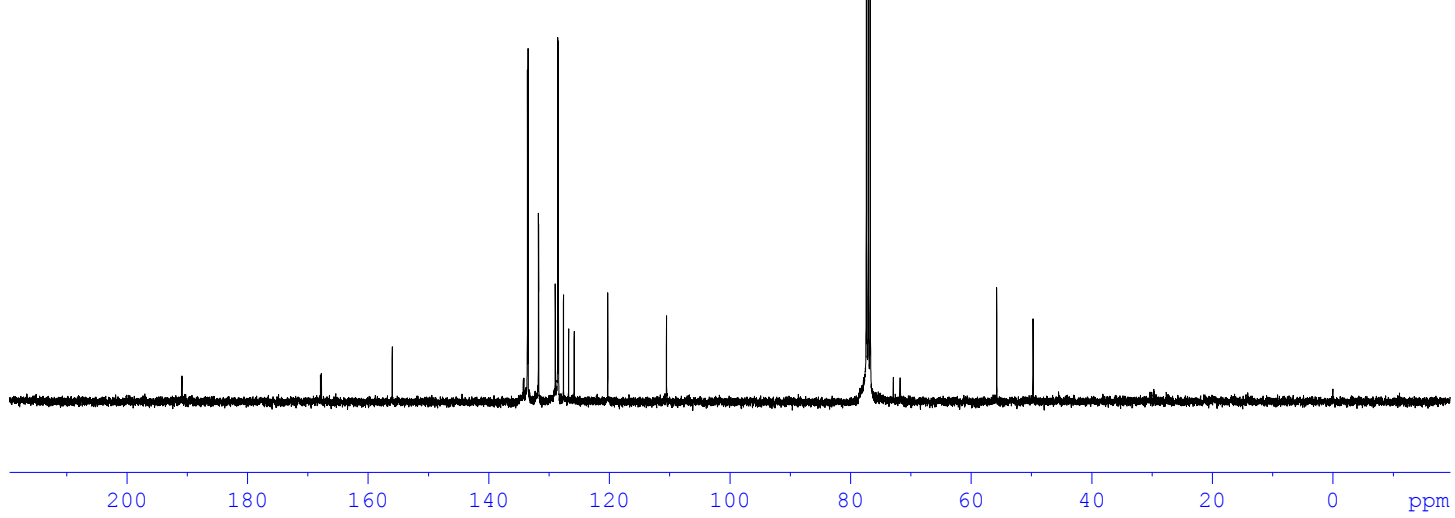

${ }^{31}$ P NMR (162 MHz, $\left.\mathrm{CDCl}_{3}\right)$

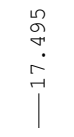<smiles>COC(=O)PC(C(=O)OC)C(=O)c1ccccc1OC</smiles>

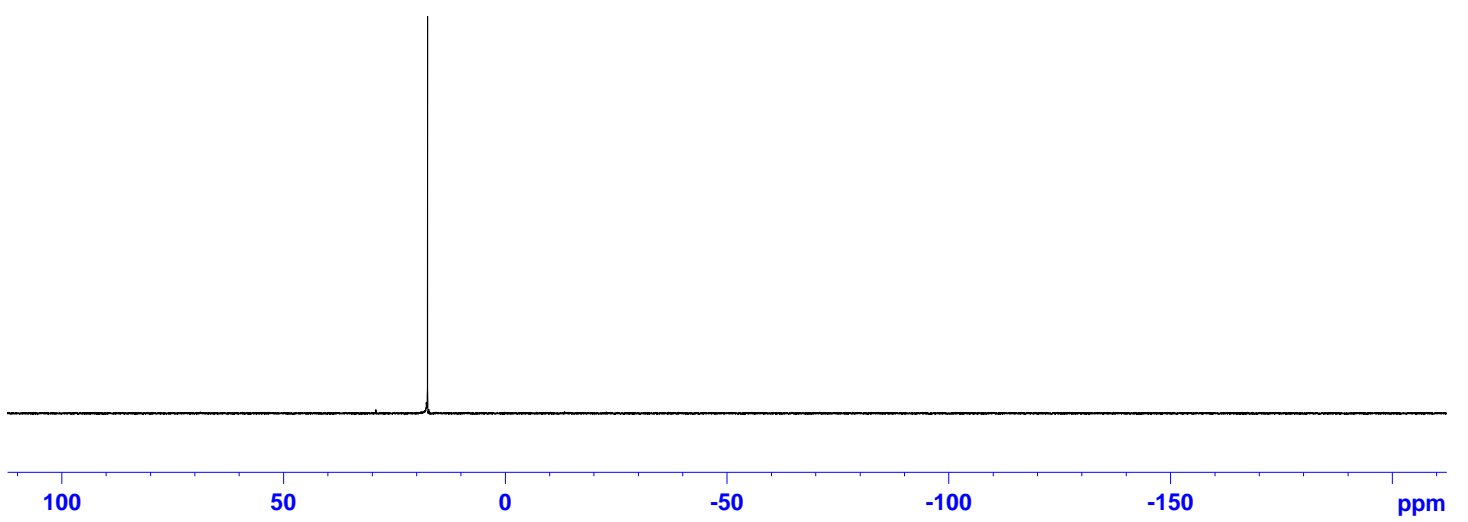


Methyl 3-(3,4-difluorophenyl)-3-oxo-2-(triphenylphosphoranylidene)propanoate 29: ${ }^{1} \mathrm{H}$ NMR (400 MHz, $\left.\mathrm{CDCl}_{3}\right)$

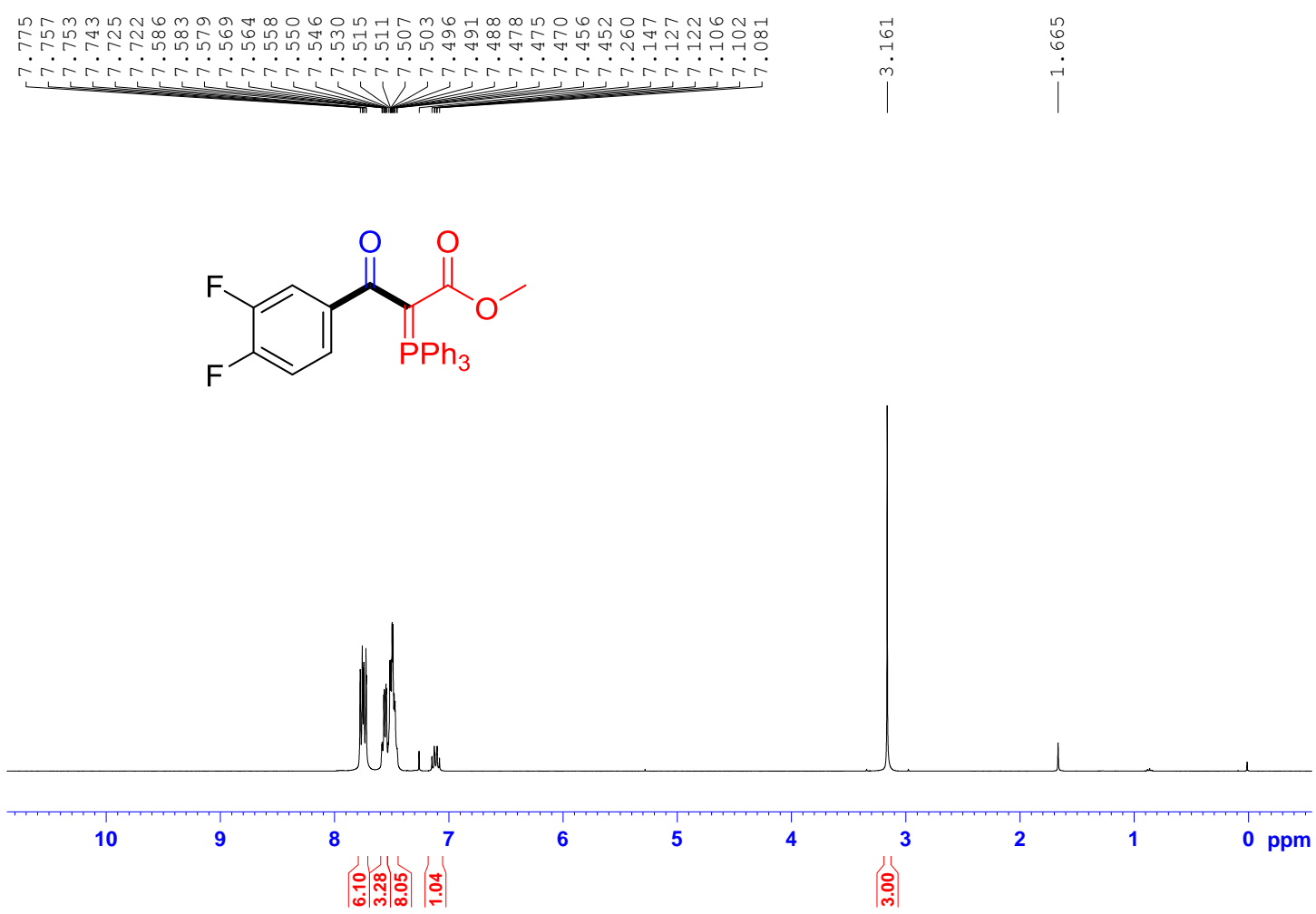

${ }^{13} \mathrm{C}$ NMR (100 MHz, CDCl3)
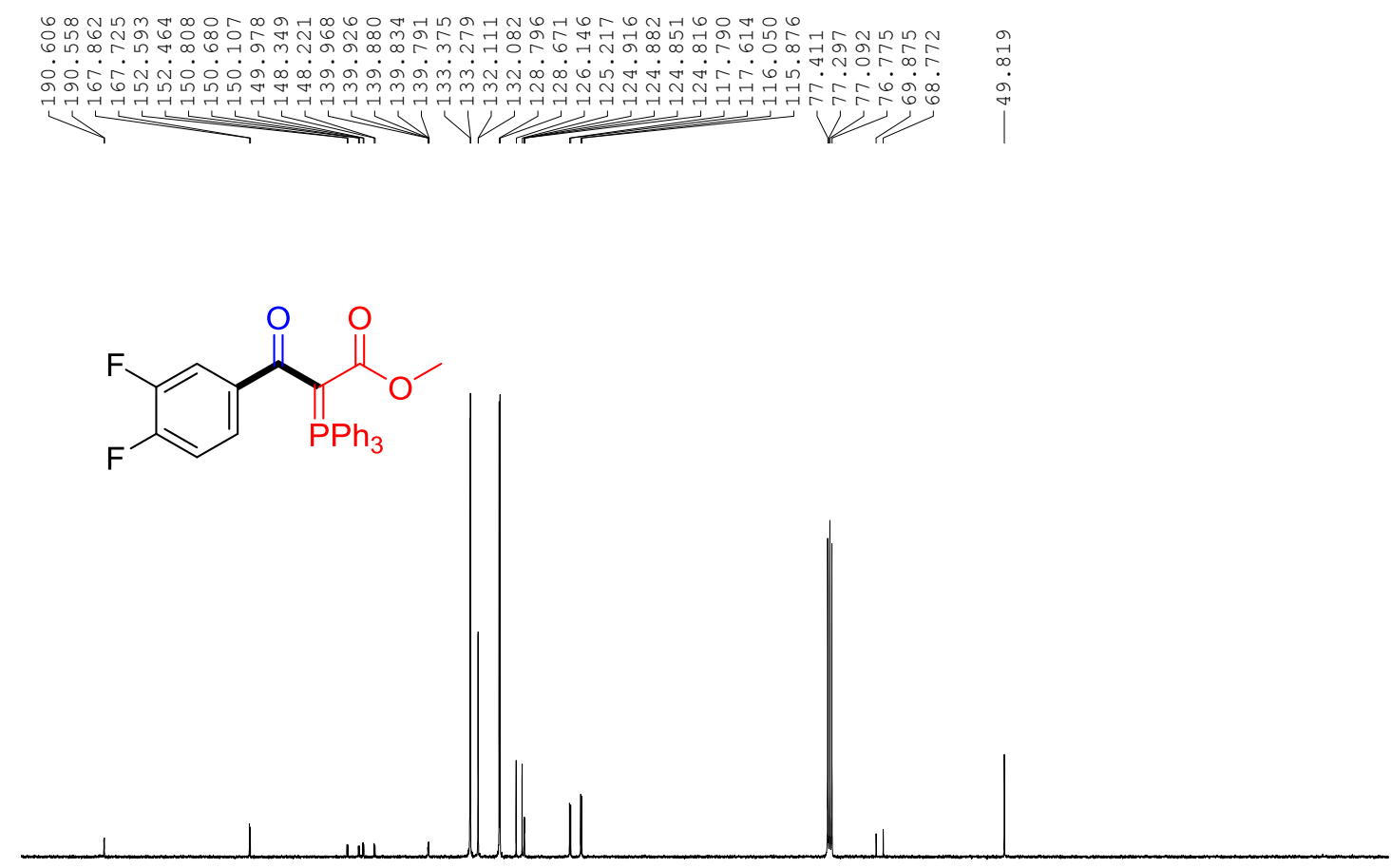

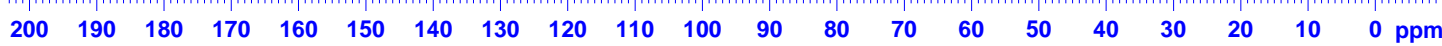


${ }^{31}$ P NMR (162 MHz, $\left.\mathrm{CDCl}_{3}\right)$<smiles>COC(=O)C(=Pc1ccccc1)C(=O)c1ccc(F)c(F)c1</smiles>

200

150

100

50

$-50$

$-100$

$-150 \mathrm{ppm}$

Methyl-3-(3,5-bis(trifluoromethyl)phenyl)-3-oxo-2-(triphenylphosphoranylidene) propanoate 30: ${ }^{1} \mathrm{H}$ NMR (400 $\left.\mathrm{MHz}, \mathrm{CDCl}_{3}\right)$

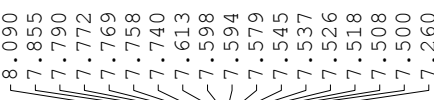<smiles>COC(=O)C(=Pc1ccccc1)C(=O)c1cc(C(F)(F)F)cc(C(F)(F)F)c1</smiles>

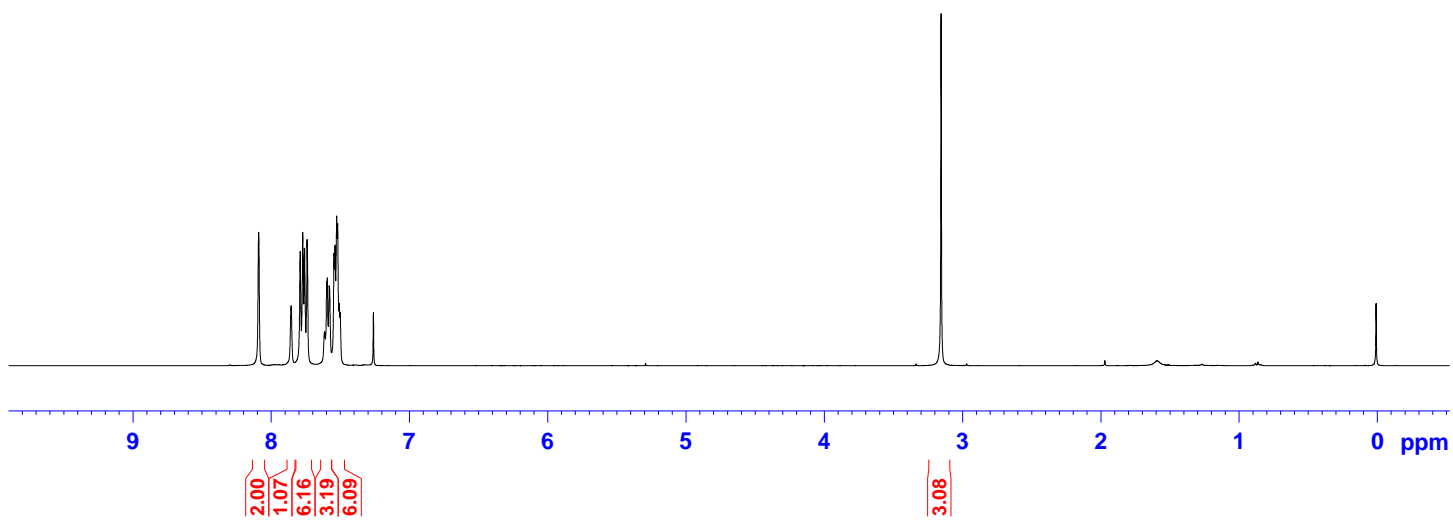




\section{${ }^{13}$ C NMR (100 MHz, $\left.\mathrm{CDCl}_{3}\right)$}
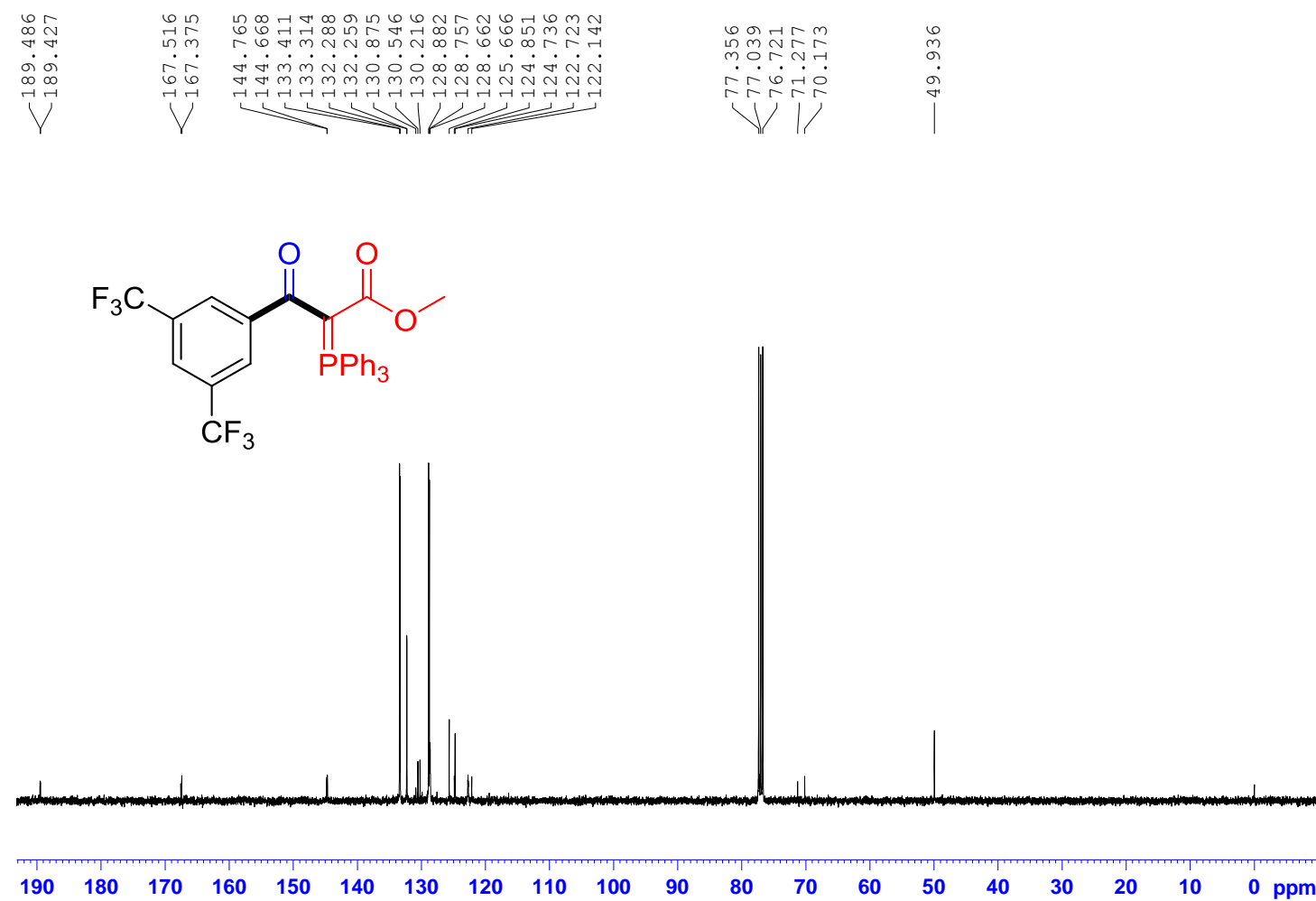

${ }^{31}$ P NMR (162 MHz, $\mathrm{CDCl}_{3}$ )

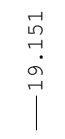<smiles>COC(=O)C(=Pc1ccccc1)C(=O)c1cc(C(F)(F)F)cc(C(F)(F)F)c1</smiles>

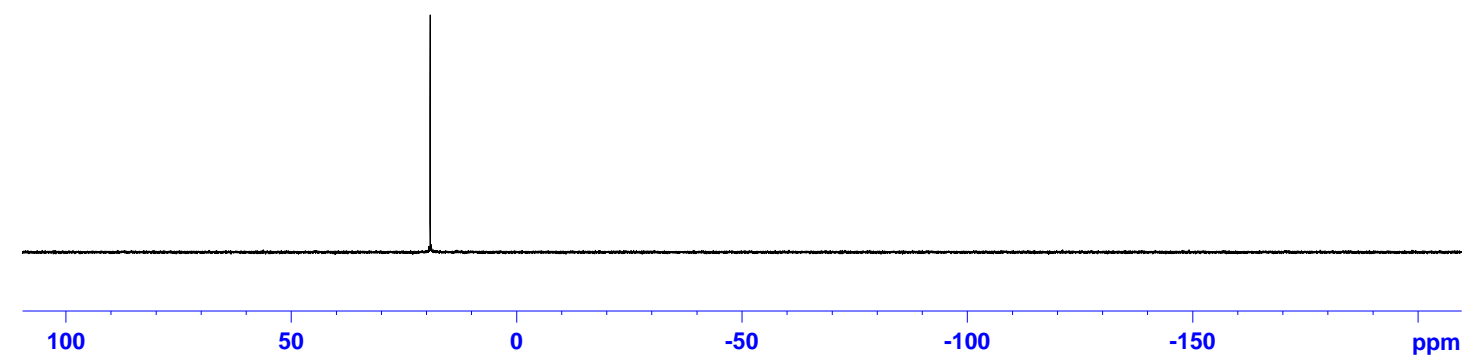


Methyl 3-(3,4-dimethylphenyl)-3-oxo-2-(triphenylphosphoranylidene)propanoate 31: ${ }^{1} \mathrm{H}$ NMR (400 MHz, $\left.\mathrm{CDCl}_{3}\right)$

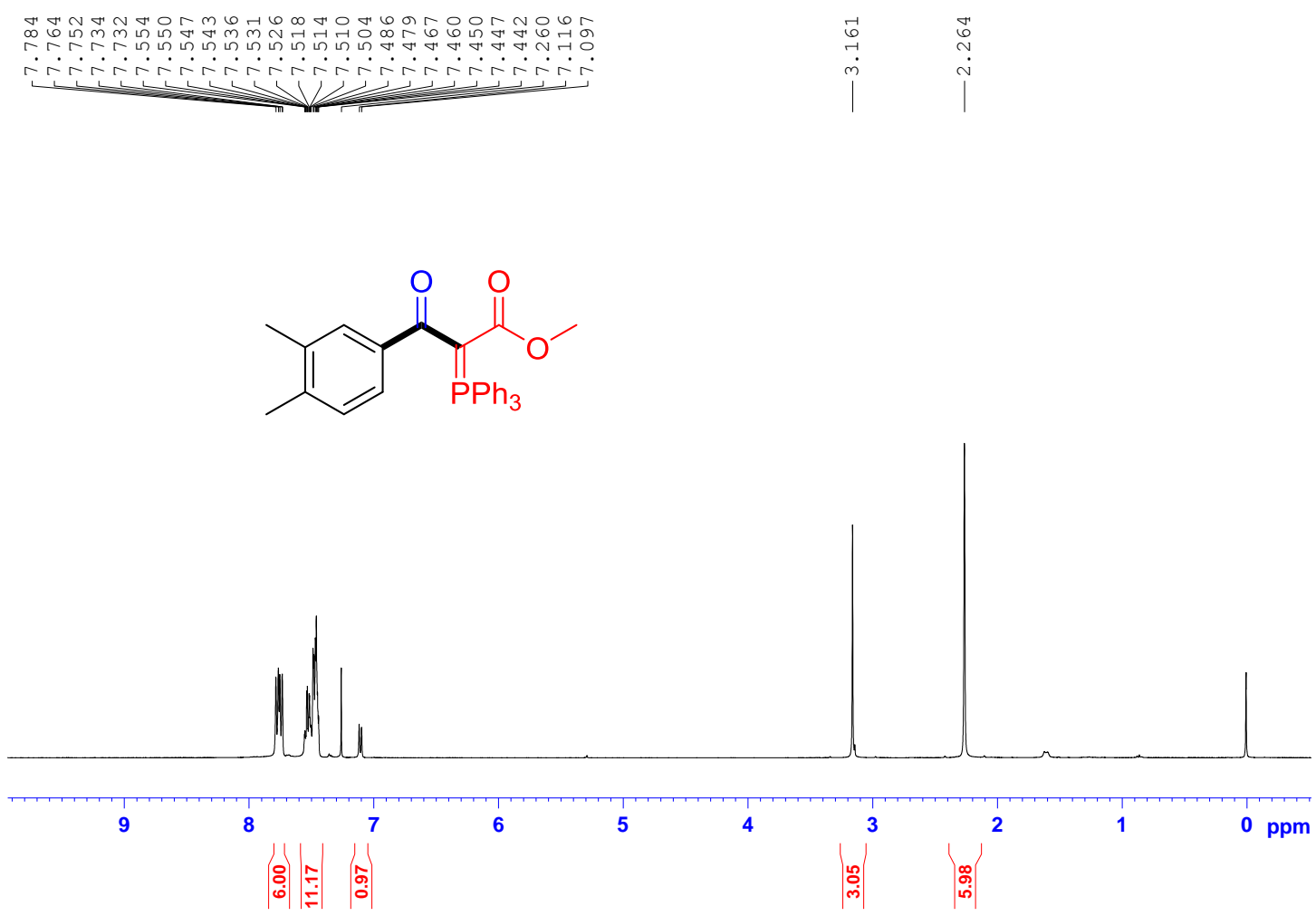

\section{${ }^{13} \mathrm{C}$ NMR (100 MHz, $\left.\mathrm{CDCl}_{3}\right)$}

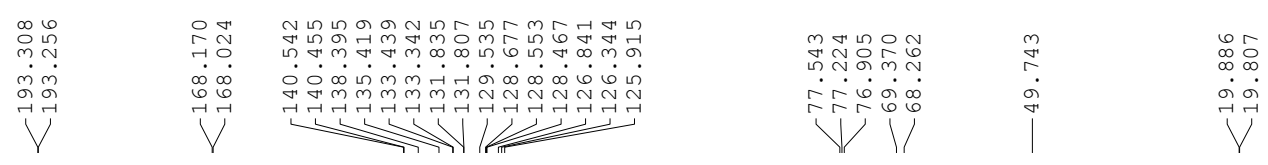<smiles>COC(=O)C(=Pc1ccccc1)C(=O)c1ccc(C)c(C)c1</smiles>

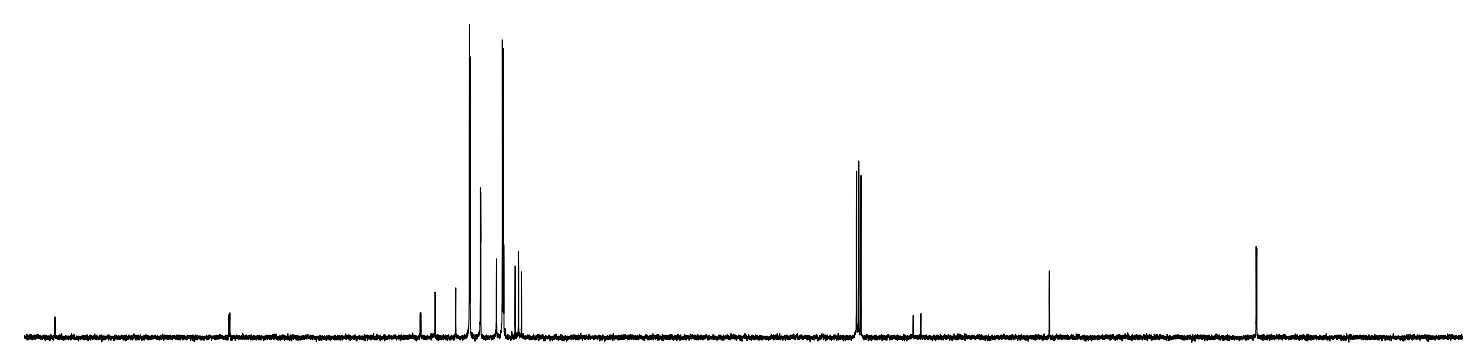

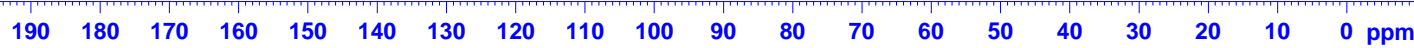


${ }^{31}$ P NMR (162 MHz, $\left.\mathrm{CDCl}_{3}\right)$<smiles>COC(=O)C(=Pc1ccccc1)C(=O)c1ccc(C)c(C)c1</smiles>

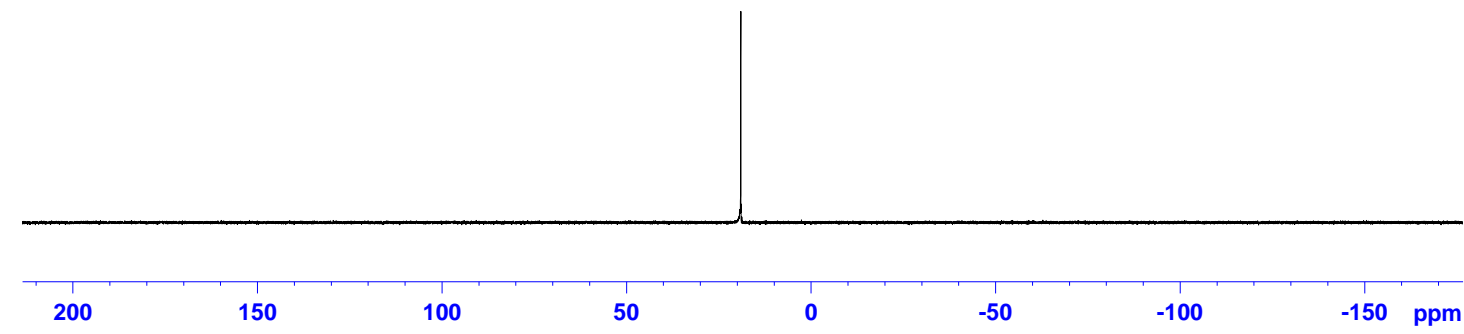

Methyl 3-(3,5-dimethylphenyl)-3-oxo-2-(triphenylphosphoranylidene)propanoate 32: ${ }^{1} \mathrm{H}$ NMR (400 $\mathrm{MHz}, \mathrm{CDCl}_{3}$ )
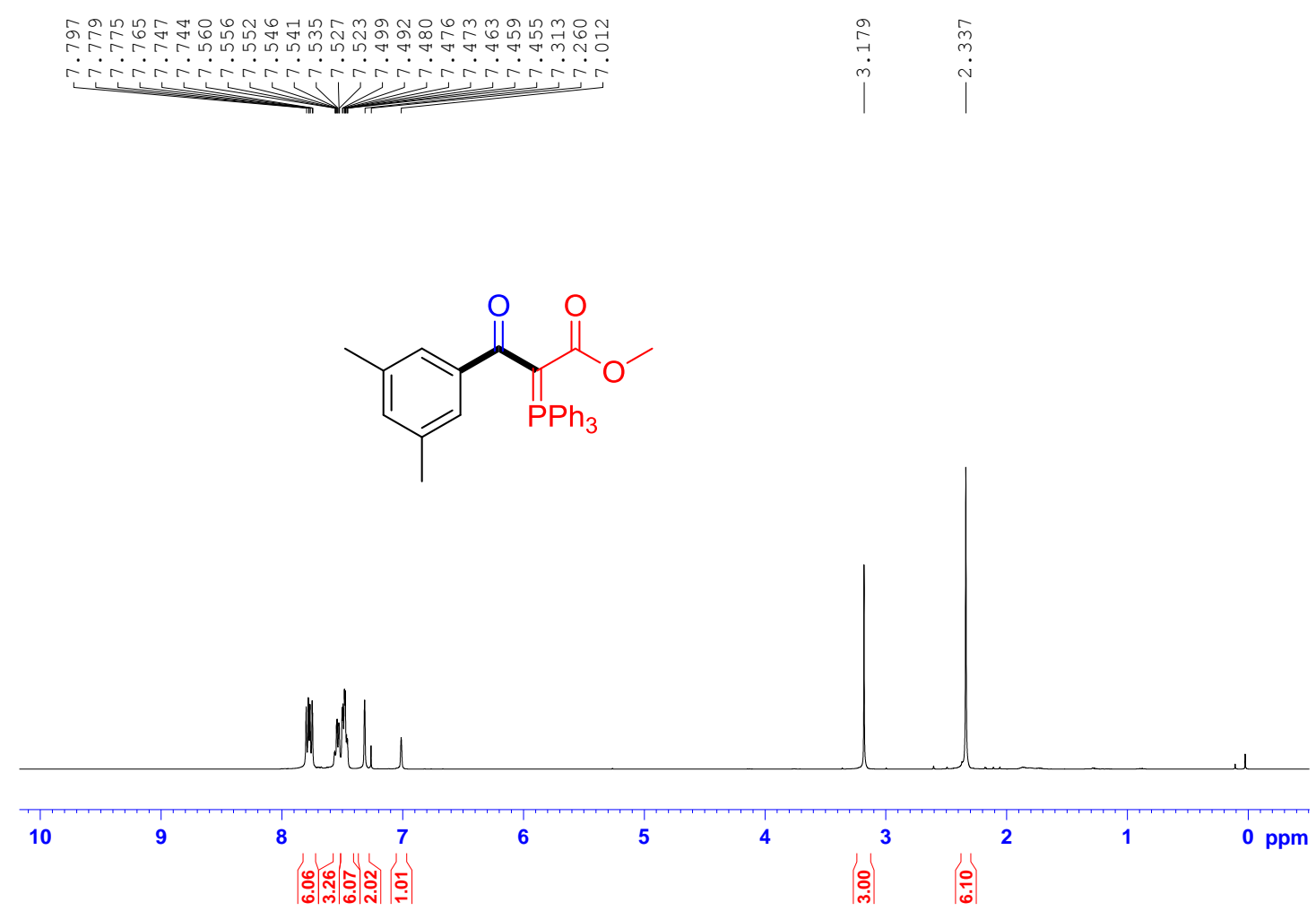


\section{${ }^{13}$ C NMR (100 MHz, $\left.\mathrm{CDCl}_{3}\right)$}
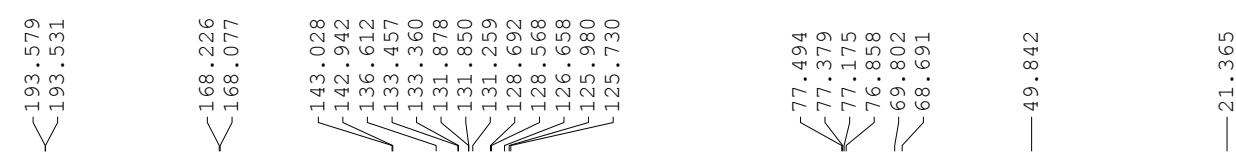<smiles>COC(=O)C(=P)C(=O)c1cc(C)cc(C)c1</smiles>

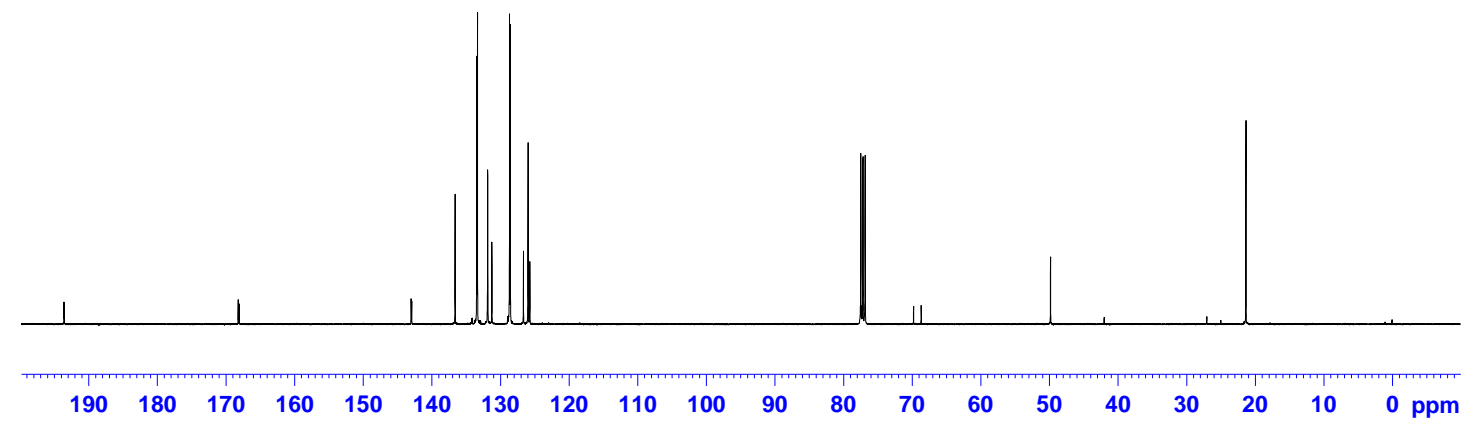

\section{${ }^{31}$ P NMR (162 MHz, $\left.\mathrm{CDCl}_{3}\right)$}<smiles>COC(=O)C(=P)C(=O)c1cc(C)cc(C)c1</smiles>

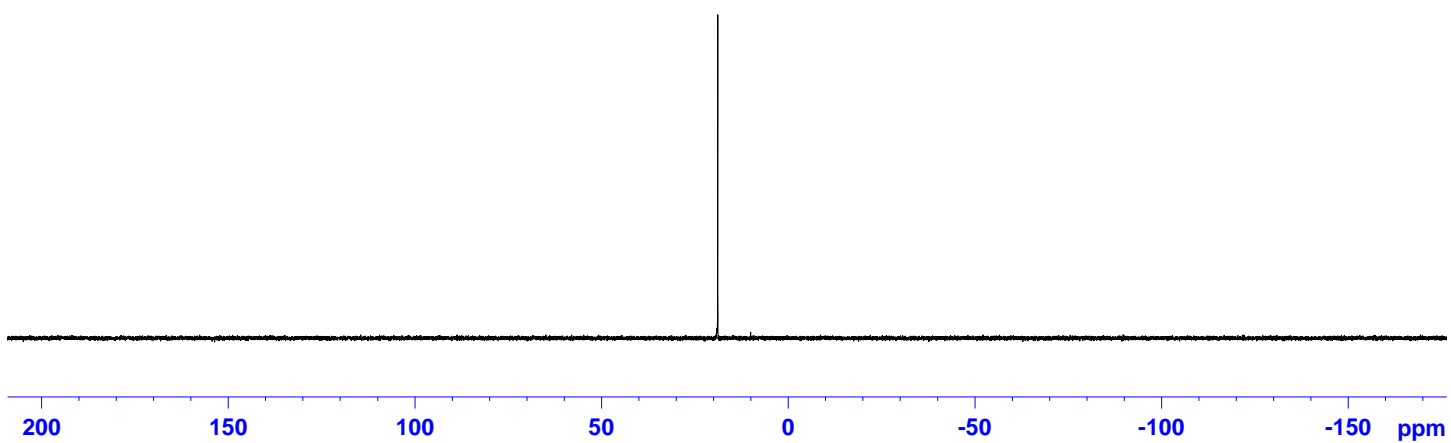


Methyl 3-(naphthalen-1-yl)-3-oxo-2-(triphenylphosphoranylidene)propanoate 33:

${ }^{1}$ H NMR (400 MHz, CDCl 3$)$
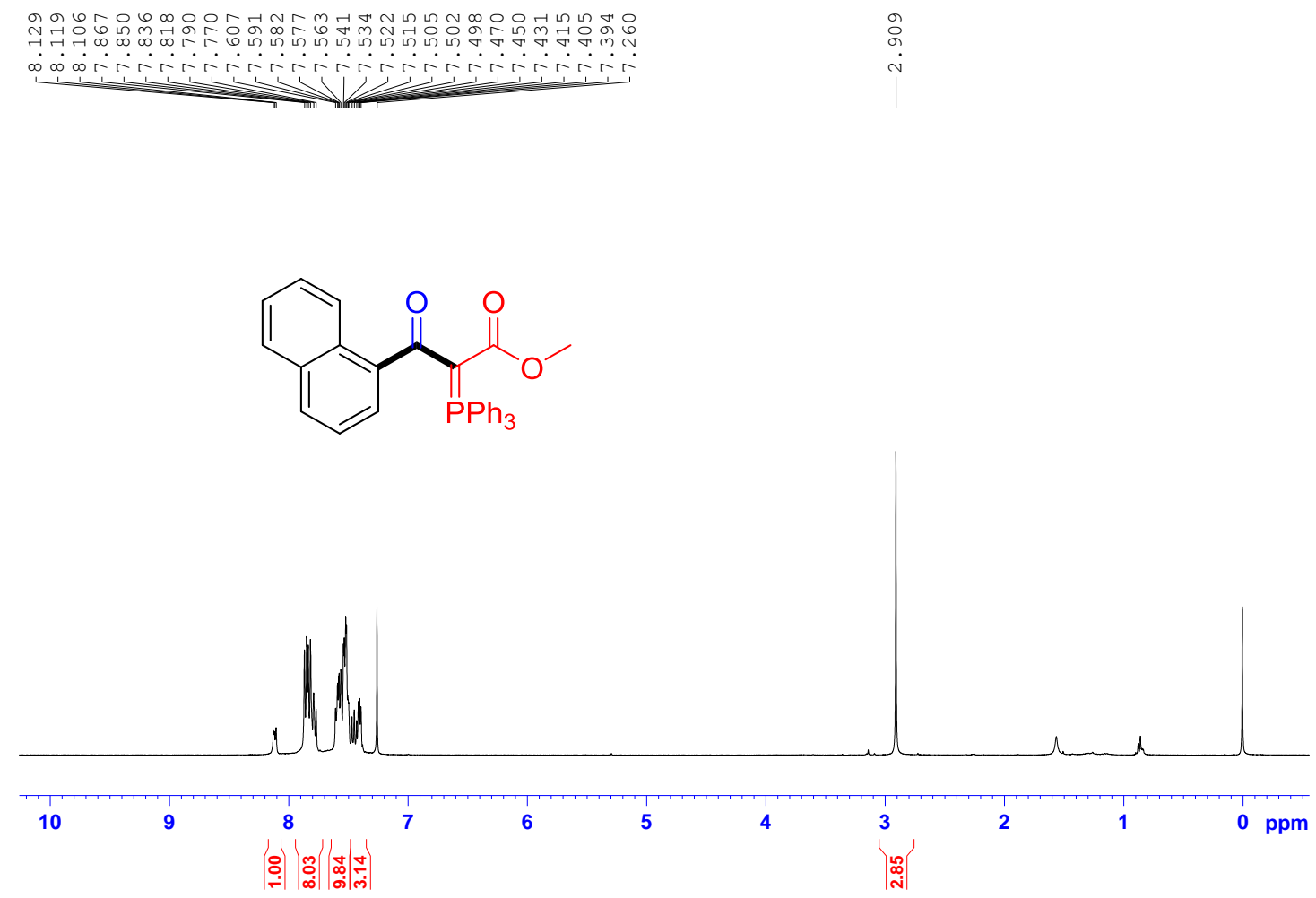

${ }^{13} \mathrm{C}$ NMR (100 MHz, $\left.\mathrm{CDCl}_{3}\right)$
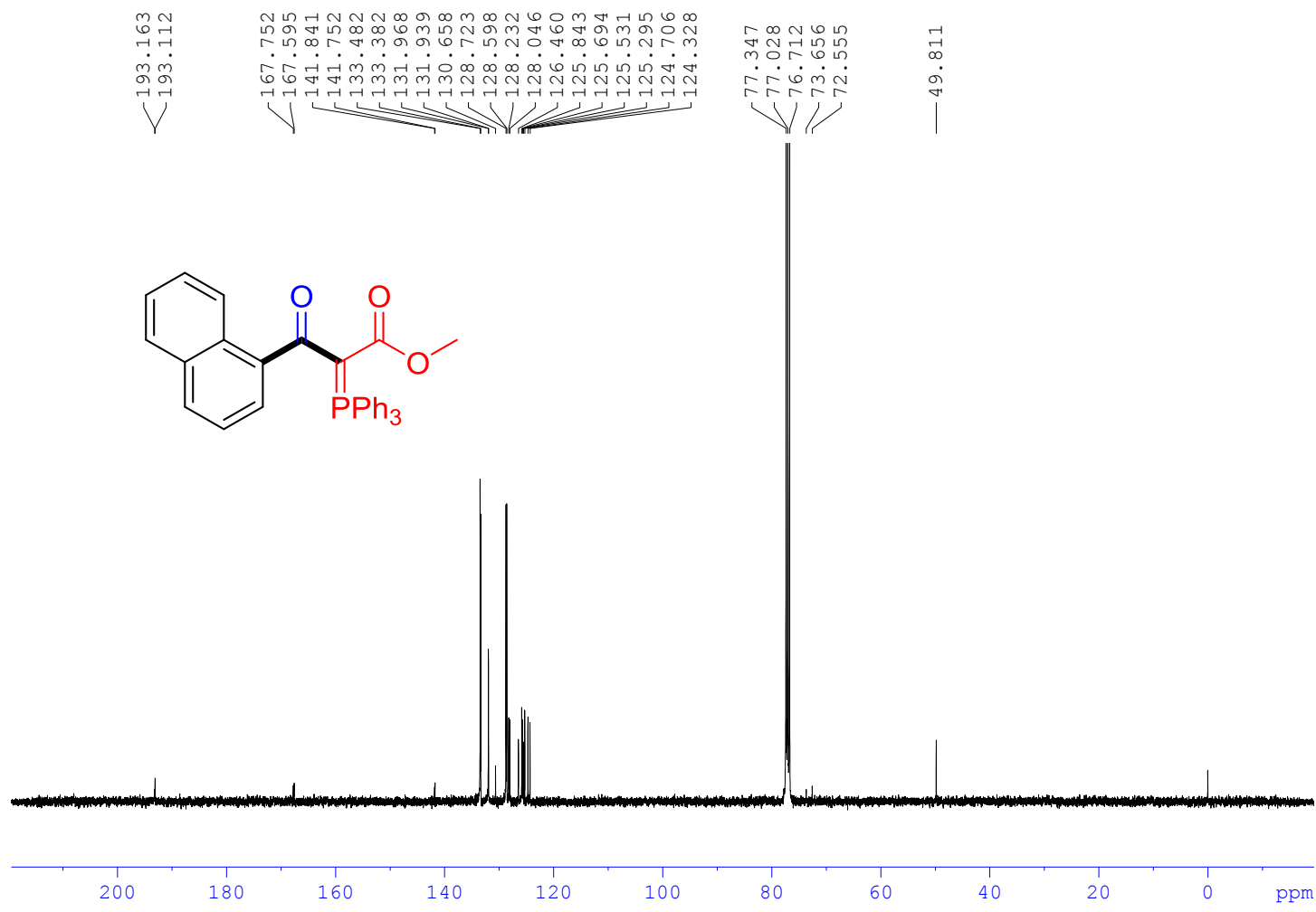
${ }^{31}$ P NMR (162 MHz, $\left.\mathrm{CDCl}_{3}\right)$<smiles>COC(=O)C(=P[PH](=O)c1ccccc1)C(=O)c1cccc2ccccc12</smiles>

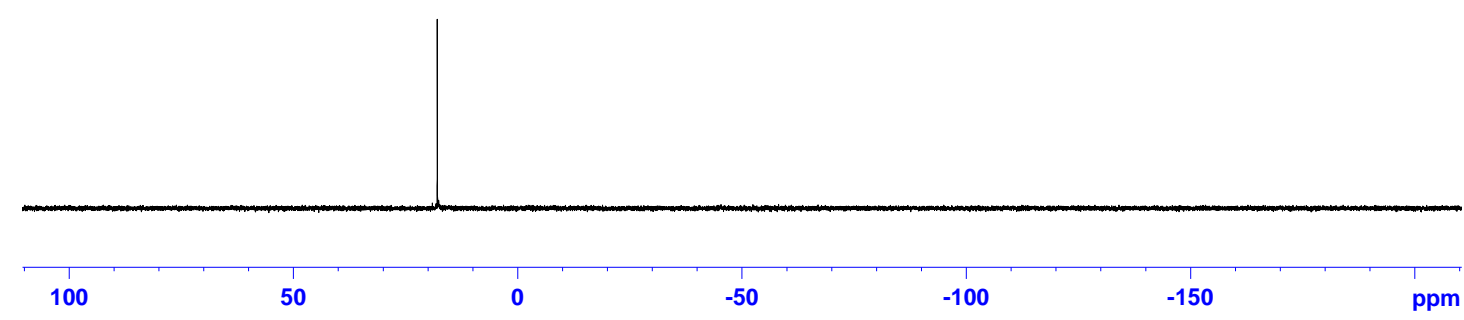

Methyl 3-oxo-3-(thiophen-2-yl)-2-(triphenylphosphoranylidene)propanoate 34:

${ }^{1}$ H NMR (400 MHz, $\left.\mathrm{CDCl}_{3}\right)$
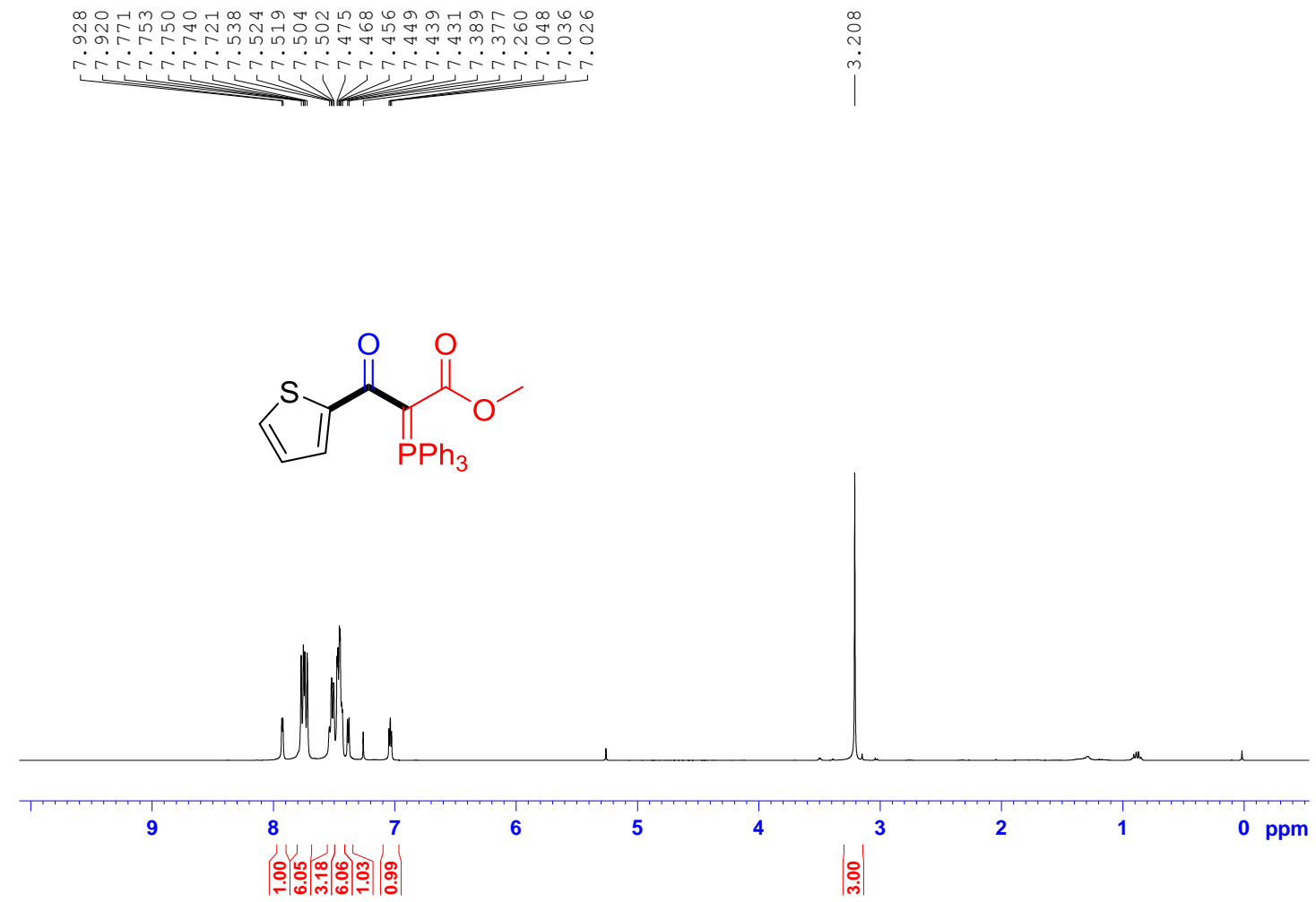
${ }^{13} \mathrm{C}$ NMR (100 MHz, $\left.\mathrm{CDCl}_{3}\right)$

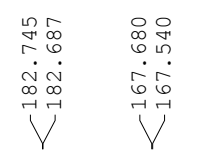

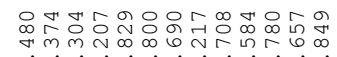

bim

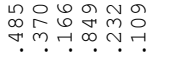

닫용

W

VI

$\infty$
$\infty$
$\dot{\sigma}$
$j$<smiles>COC(=O)C(=P)C(=O)c1cccs1</smiles>

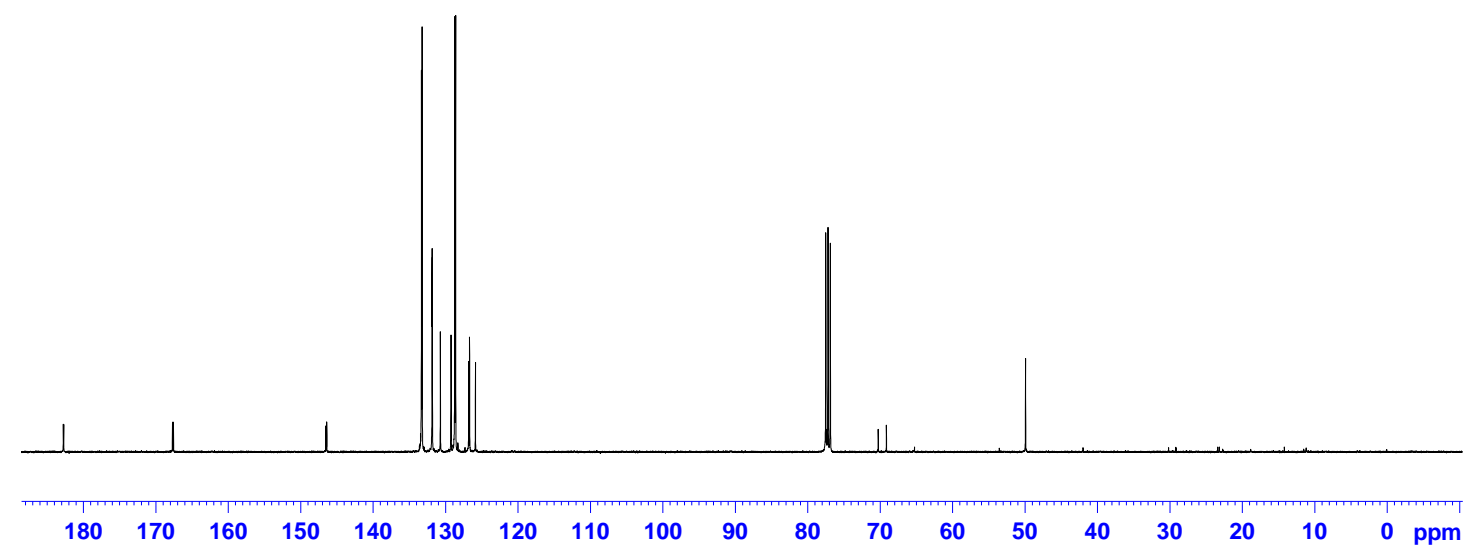

${ }^{31}$ P NMR (162 MHz, $\mathrm{CDCl}_{3}$ )<smiles>COC(=O)C(=Pc1ccccc1)C(=O)c1cccs1</smiles>

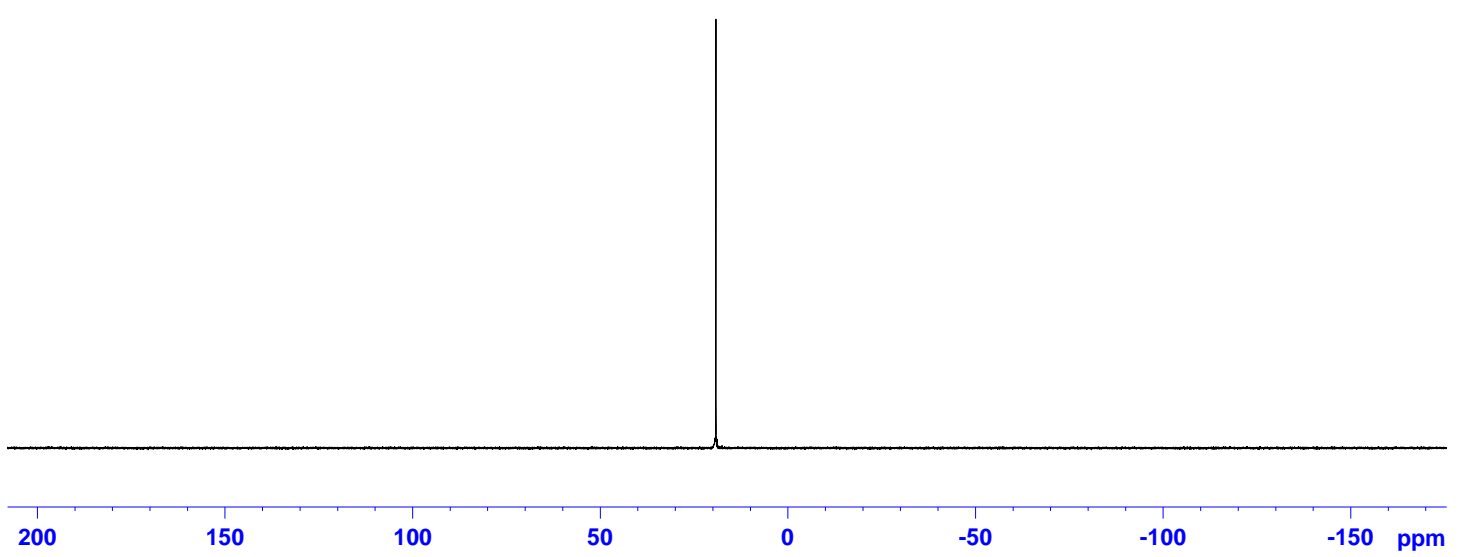


Methyl-3-(6-bromopyridin-3-yl)-3-oxo-2-(triphenylphosphoranylidene)propanoa -te 35: ${ }^{1} \mathrm{H}$ NMR (400 MHz, $\left.\mathrm{CDCl}_{3}\right)$

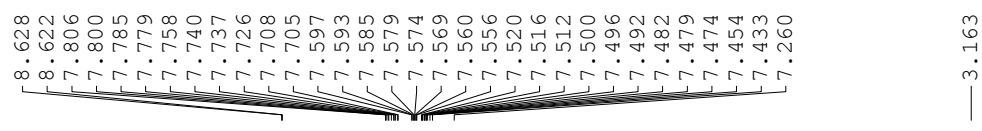<smiles>COC(=O)C(=Pc1ccccc1)C(=O)c1ccc(Br)nc1</smiles>

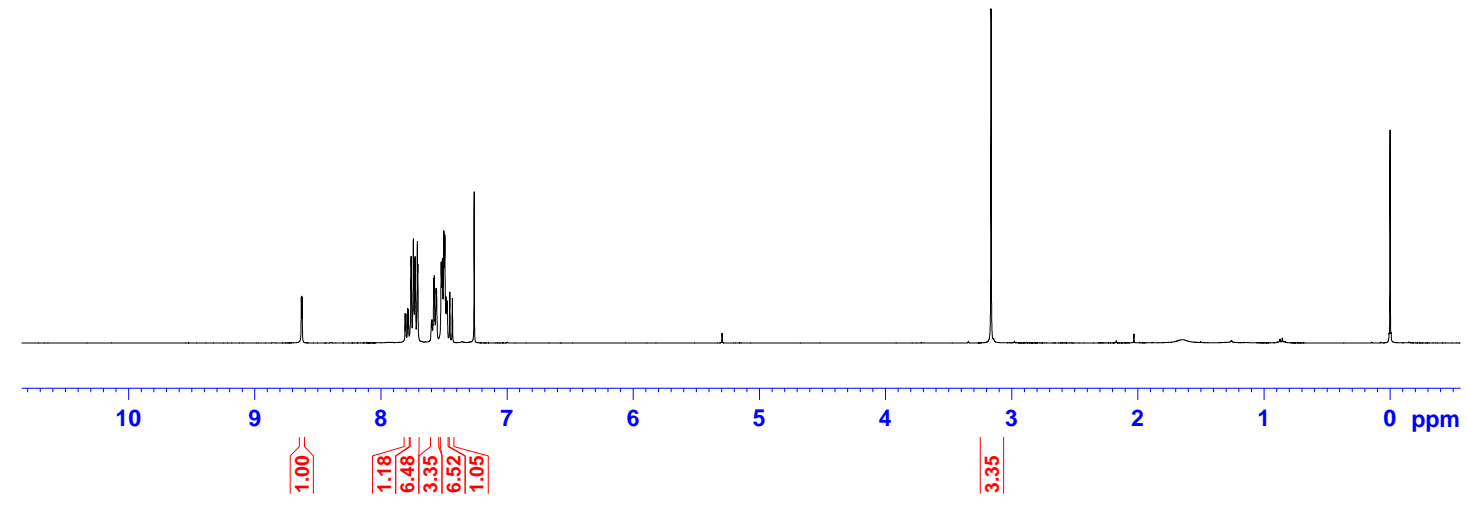

${ }^{13} \mathrm{C}$ NMR (100 MHz, $\left.\mathrm{CDCl}_{3}\right)$

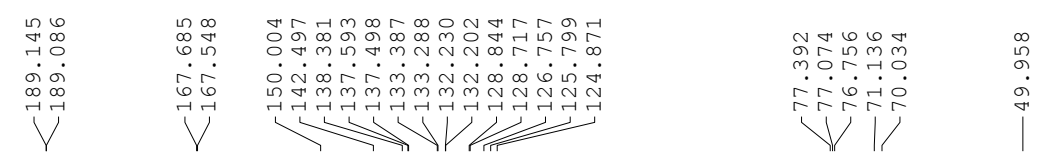

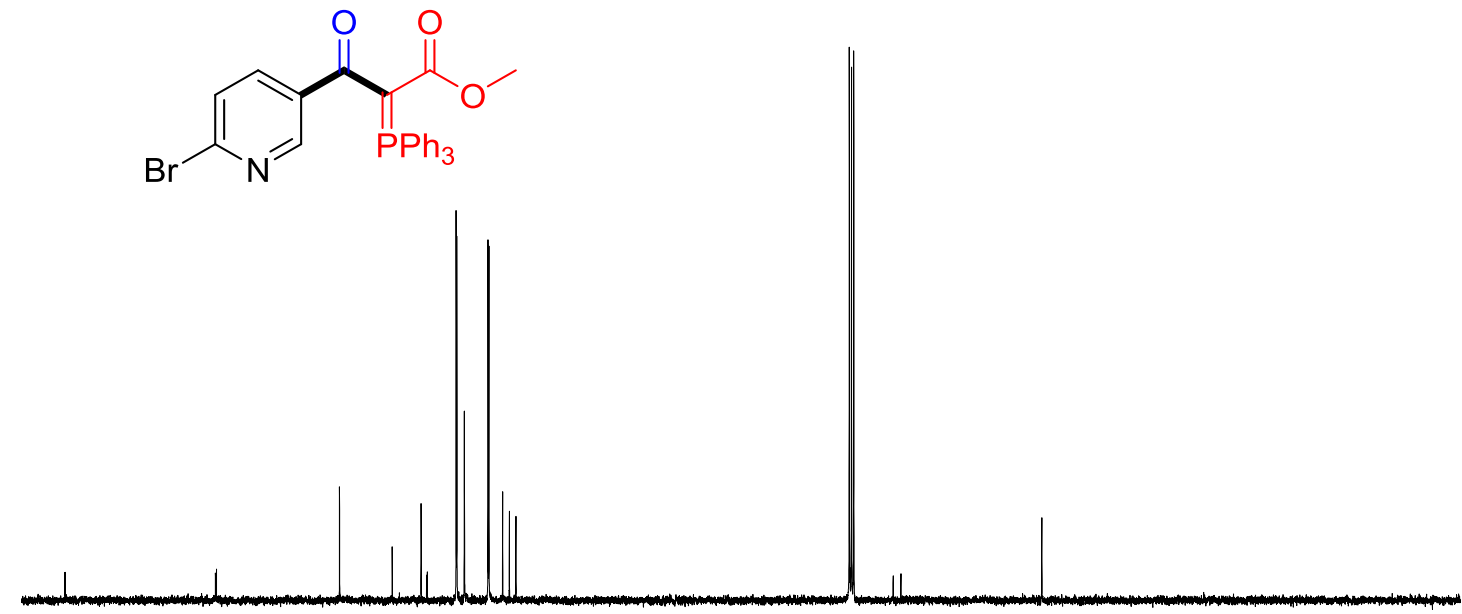

\begin{tabular}{llllllllllllllllllll}
190 & 180 & 170 & 160 & 150 & 140 & 130 & 120 & 110 & 100 & 90 & 80 & 70 & 60 & 50 & 40 & 30 & 20 & 10 & 0 \\
\hline
\end{tabular} 
${ }^{31}$ P NMR (162 MHz, $\left.\mathrm{CDCl}_{3}\right)$<smiles>COC(=O)C(=Pc1ccccc1)C(=O)c1ccc(Br)nc1</smiles>

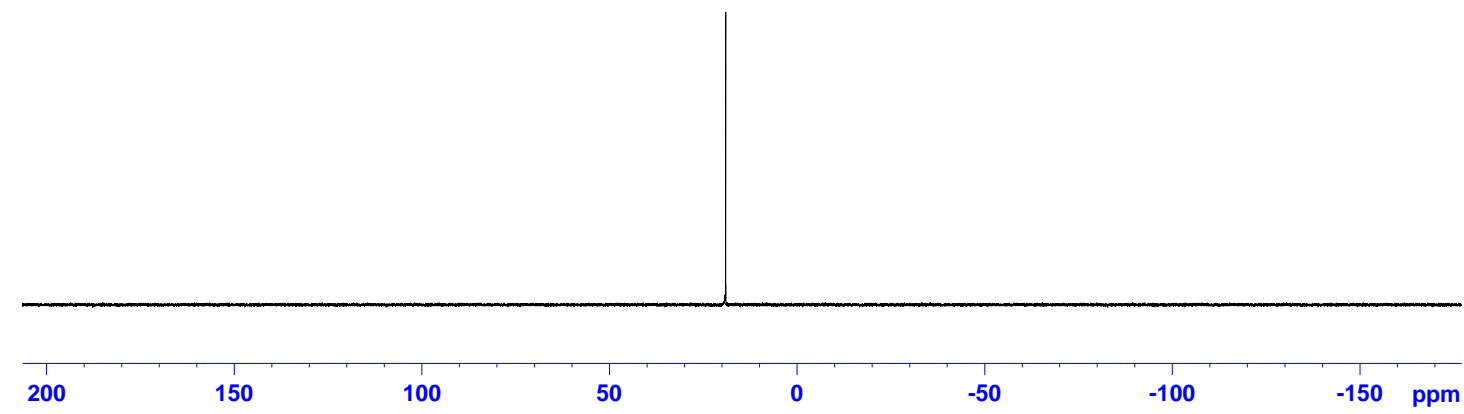

Methyl-3-(6-chloropyridin-3-yl)-3-oxo-2-(triphenylphosphoranylidene)propanoat -e 36: ${ }^{1} \mathrm{H}$ NMR (400 $\left.\mathrm{MHz}, \mathrm{CDCl}_{3}\right)$

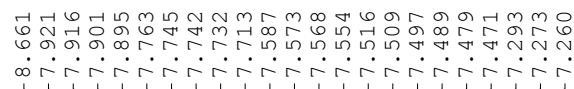<smiles>COC(=O)C(=Pc1ccccc1)C(=O)c1ccc(Cl)nc1</smiles>

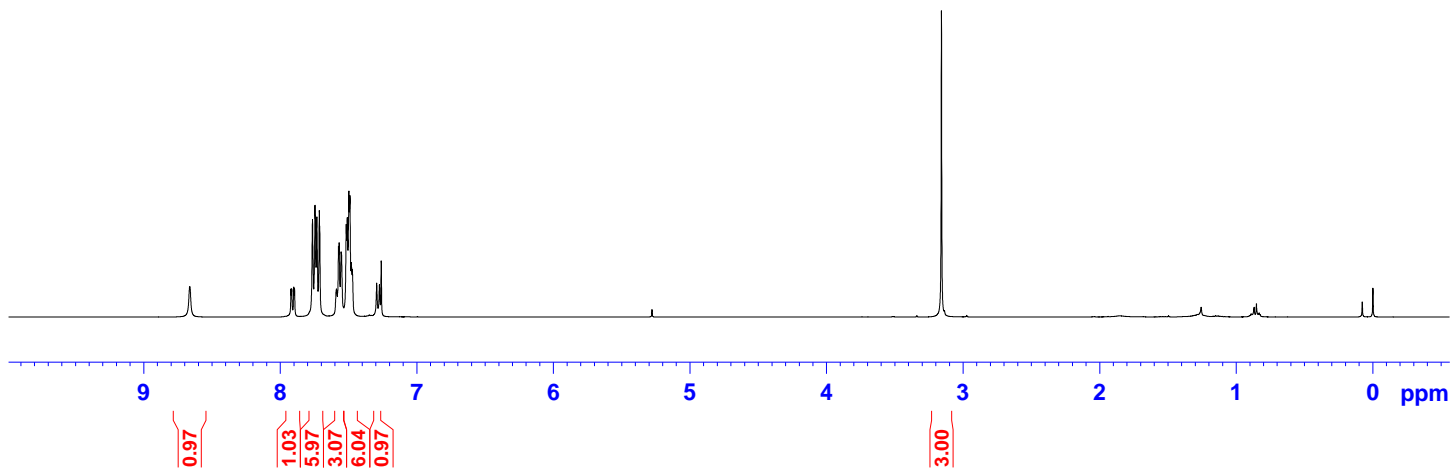




\section{${ }^{13}$ C NMR (100 MHz, $\left.\mathrm{CDCl}_{3}\right)$}
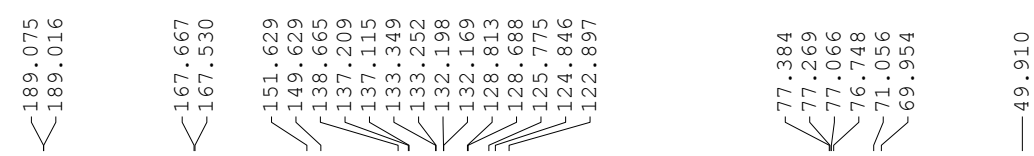<smiles>COC(=O)C(=Pc1ccccc1)C(=O)c1ccc(Cl)nc1</smiles>

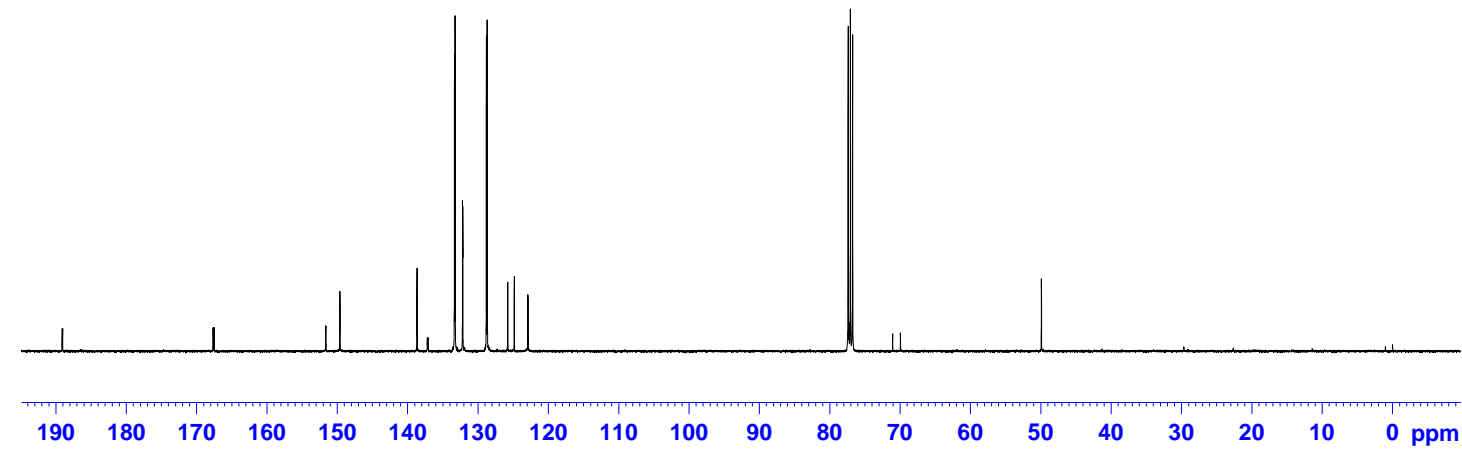

${ }^{31}$ P NMR (162 MHz, $\mathrm{CDCl}_{3}$ )

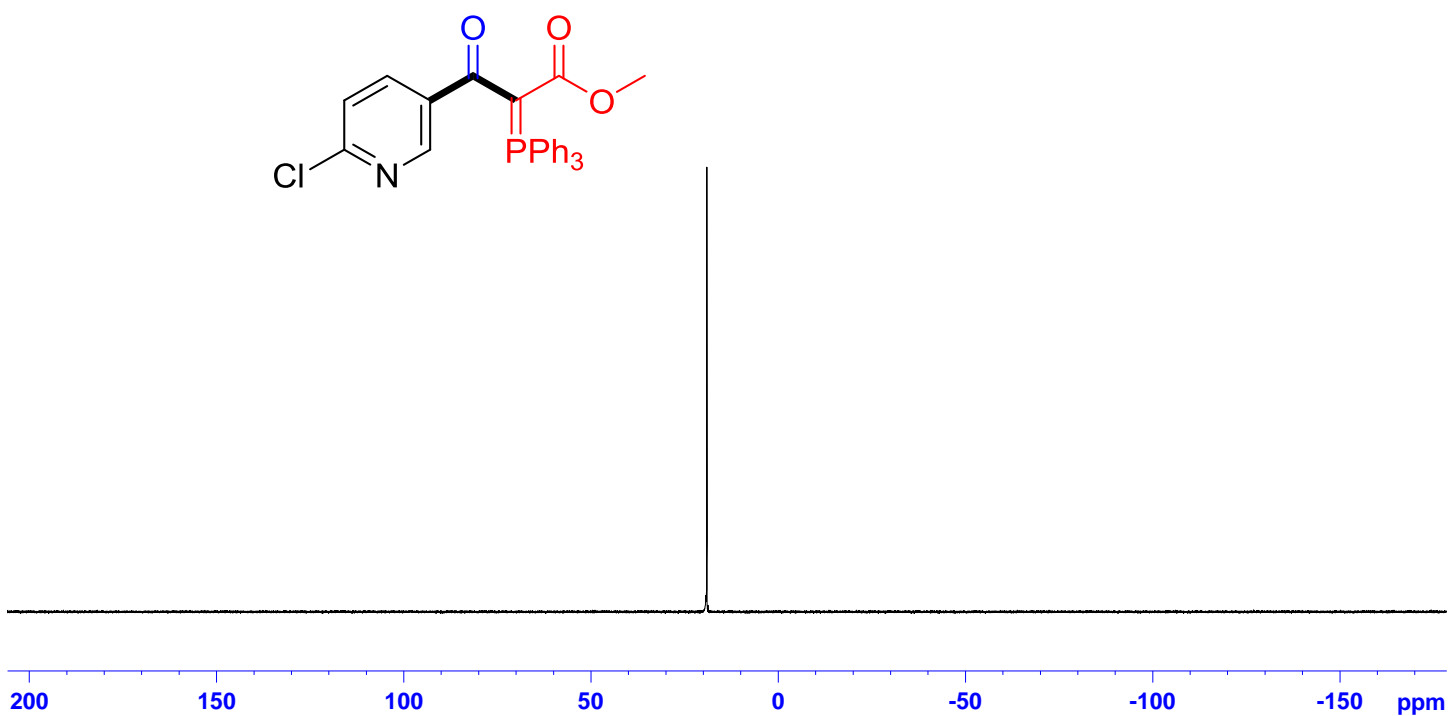


Methyl-3-(2-chloropyridin-4-yl)-3-oxo-2-(triphenylphosphoranylidene)propanoat -e 37: ${ }^{1} \mathrm{H}$ NMR (600 MHz, $\left.\mathrm{CDCl}_{3}\right)$<smiles>COC(=O)C(=P)C(=O)c1ccnc(Cl)c1</smiles>

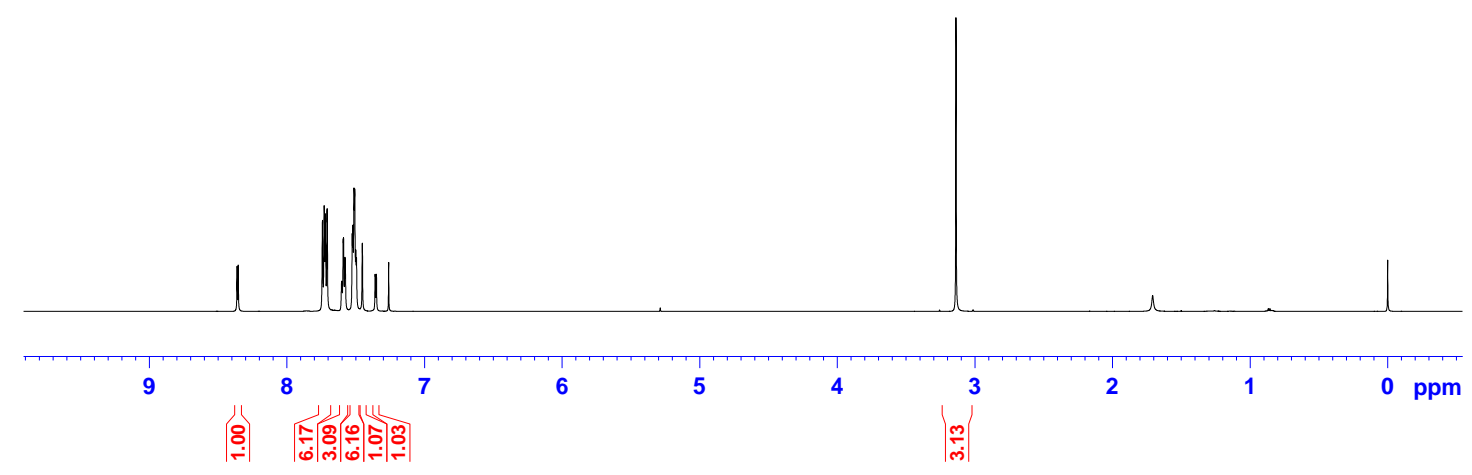

\section{${ }^{13} \mathrm{C}$ NMR (150 MHz, $\left.\mathrm{CDCl}_{3}\right)$}
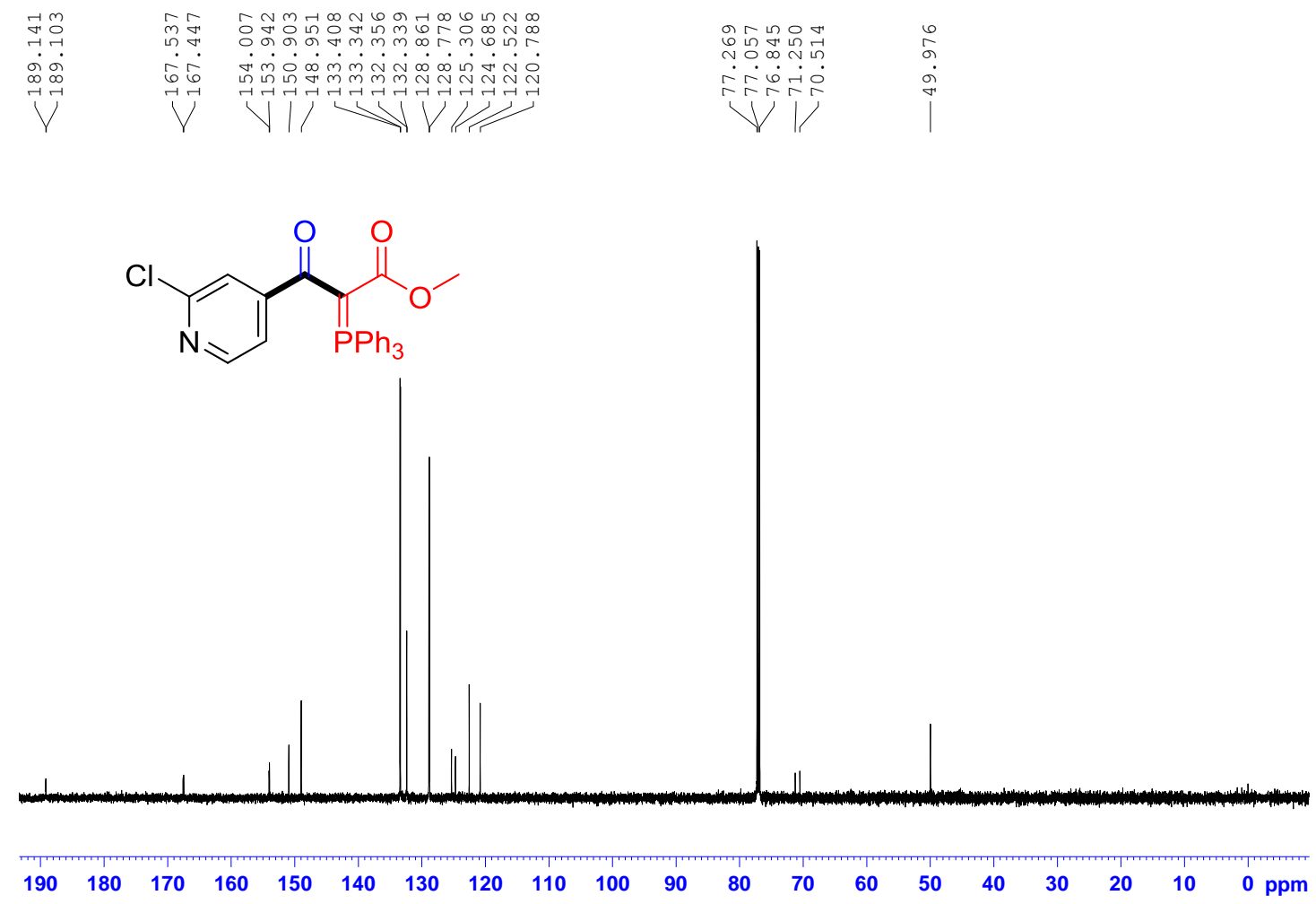
${ }^{31}$ P NMR (162 MHz, $\left.\mathrm{CDCl}_{3}\right)$<smiles>COC(=O)C(=Pc1ccccc1)C(=O)c1ccnc(Cl)c1</smiles>

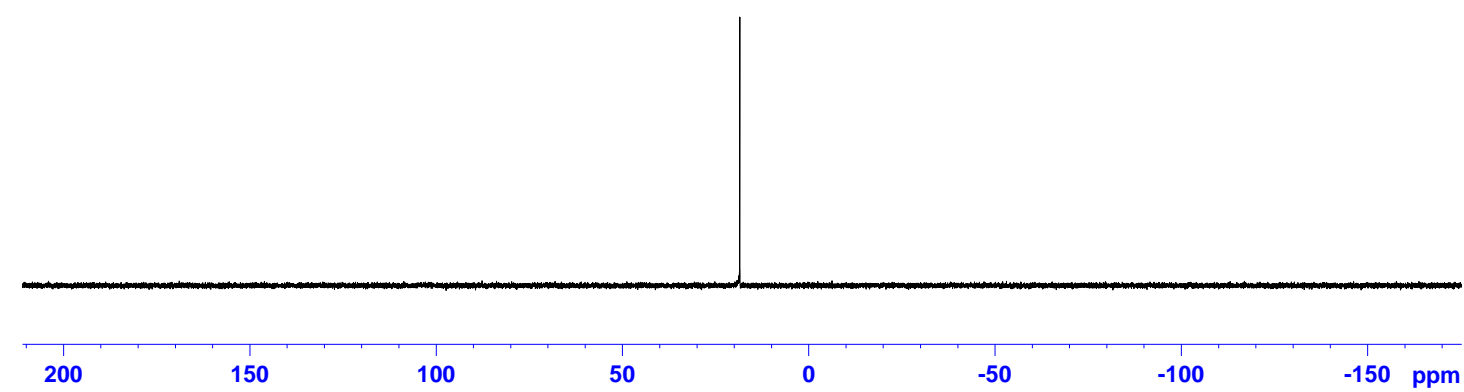

Methyl 4-(3-ethoxy-3-oxo-2-(triphenylphosphoranylidene)propanoyl)benzoate 38:

${ }^{1} \mathrm{H}$ NMR (400 MHz, $\mathrm{CDCl}_{3}$ )
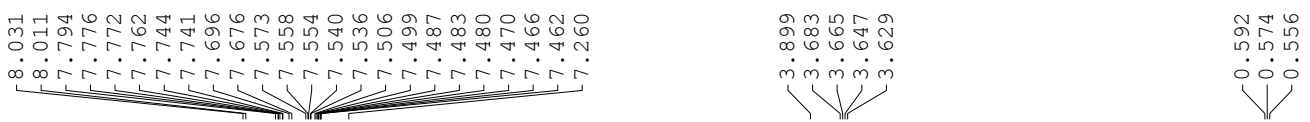<smiles>CCOC(=O)C(=P)C(=O)c1ccc(C(=O)OC)cc1</smiles>

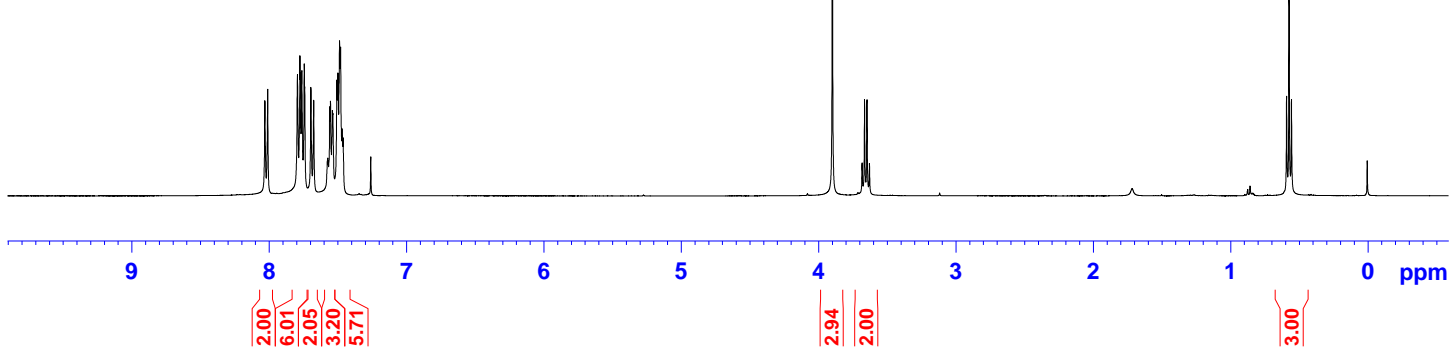




\section{${ }^{13} \mathrm{C}$ NMR (100 MHz, $\left.\mathrm{CDCl}_{3}\right)$}

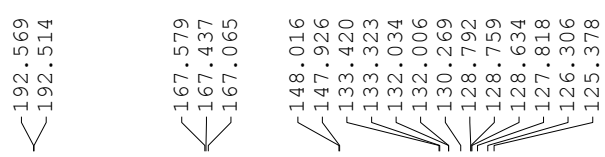<smiles>CCOC(=O)C(=P)C(=O)c1ccc(C(=O)OC)cc1</smiles>
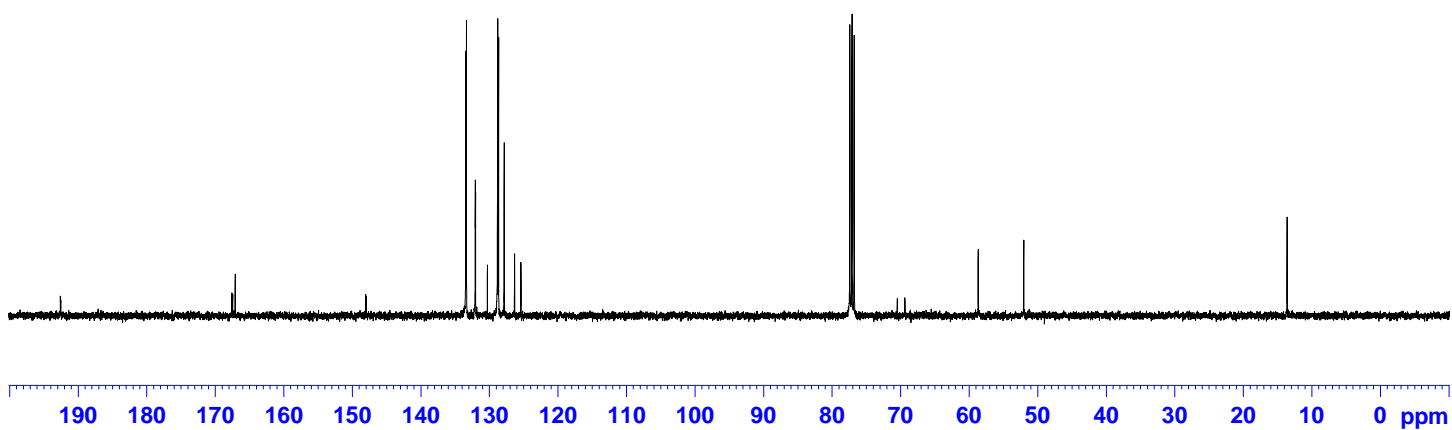

\section{${ }^{31}$ P NMR (162 MHz, $\left.\mathrm{CDCl}_{3}\right)$}<smiles>CCOC(=O)C(=P)C(=O)c1ccc(C(=O)OC)cc1</smiles>

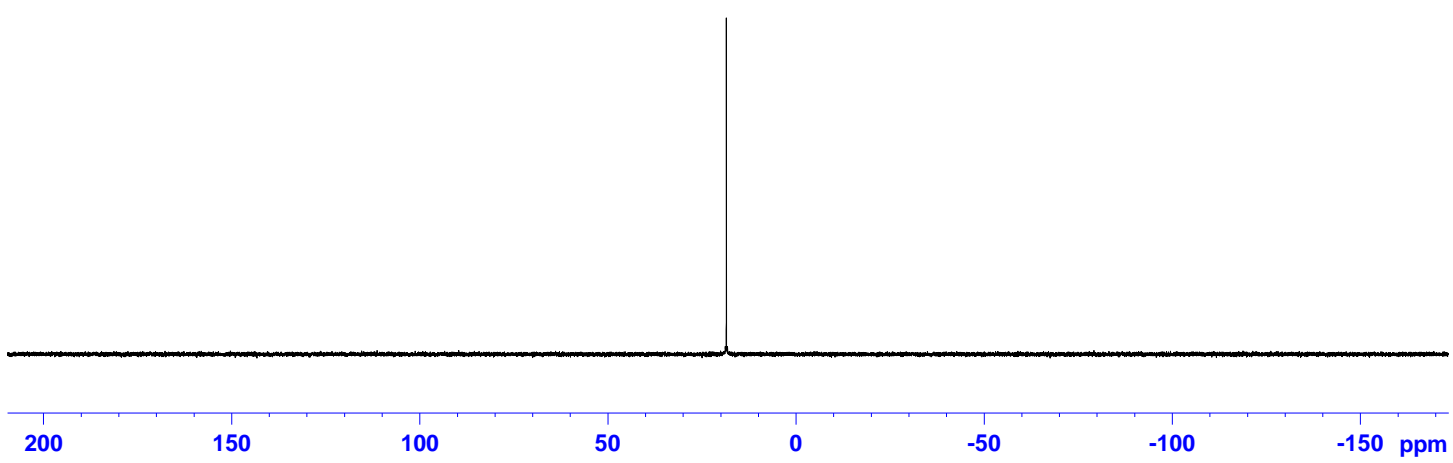


Methyl-4-(3-(benzyloxy)-3-oxo-2-(triphenylphosphoranylidene)propanoyl)benzoate 39: ${ }^{1} \mathrm{H}$ NMR (400 MHz, $\left.\mathrm{CDCl}_{3}\right)$

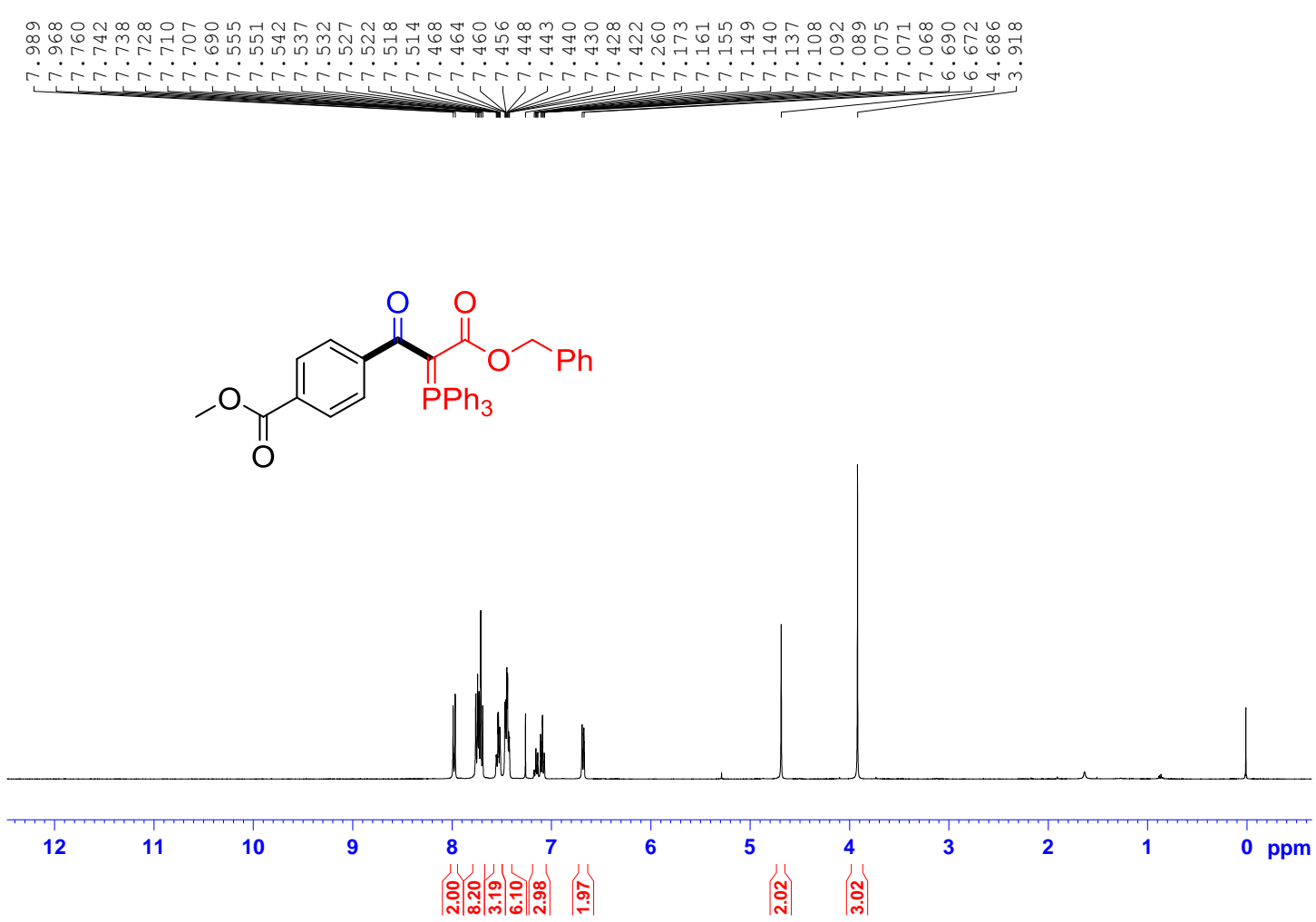

\section{${ }^{13} \mathrm{C}$ NMR (100 MHz, $\left.\mathrm{CDCl}_{3}\right)$}
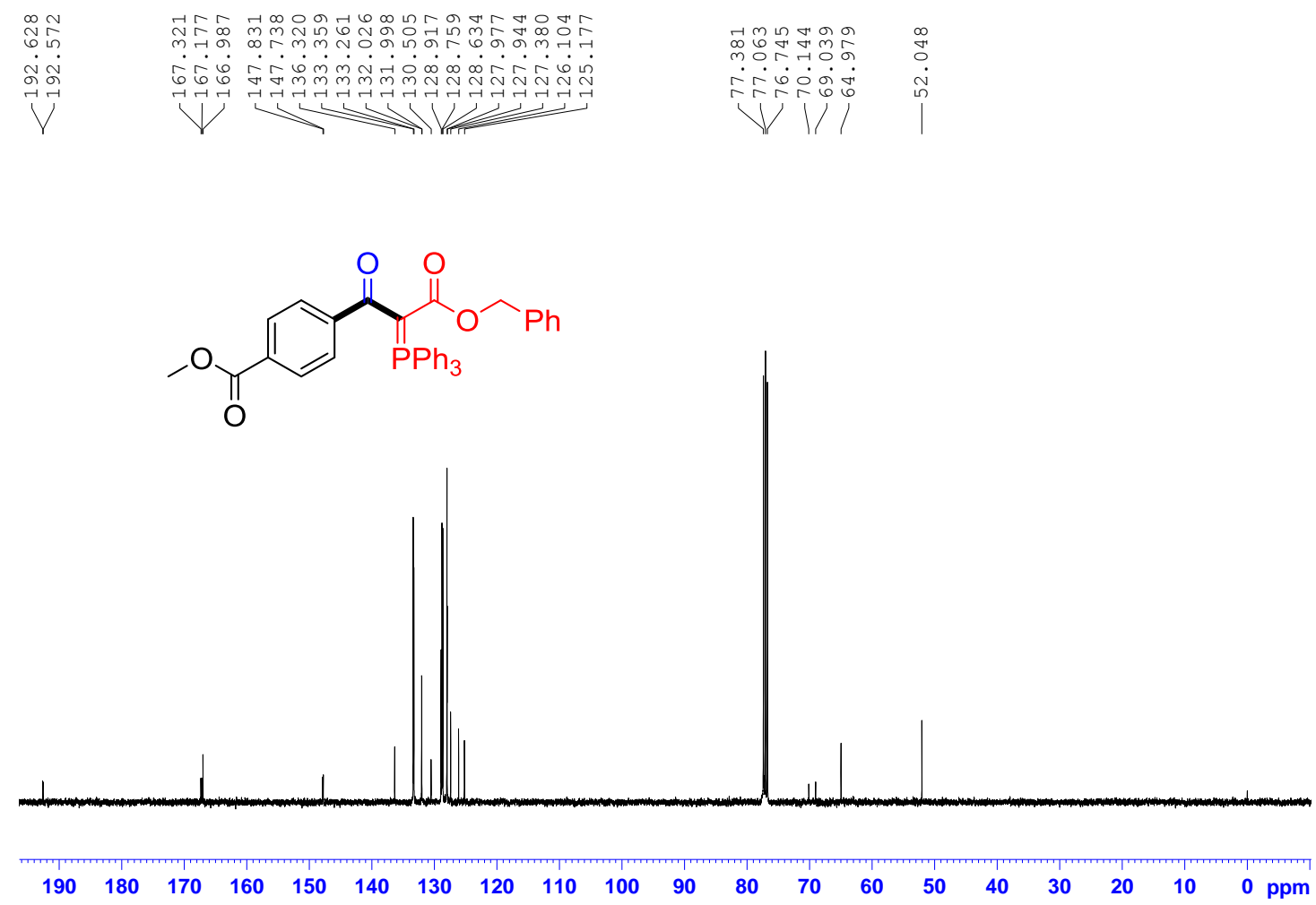
${ }^{31}$ P NMR (162 MHz, $\left.\mathrm{CDCl}_{3}\right)$

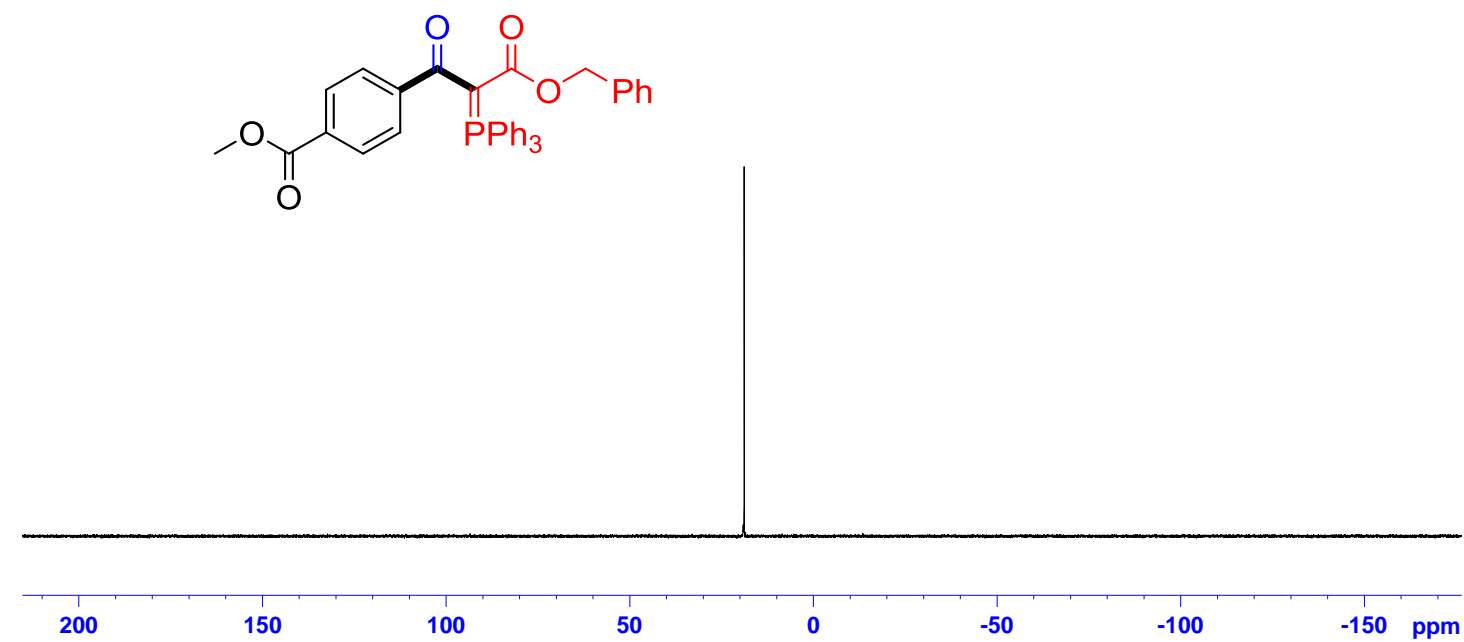

Methyl 4-(2-cyano-2-(triphenylphosphoranylidene)acetyl)benzoate 40:

${ }^{1}$ H NMR (400 MHz, $\left.\mathrm{CDCl}_{3}\right)$

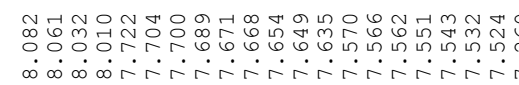<smiles>COC(=O)c1ccc(C(=O)C(=P)C(C#N)Pc2ccccc2)cc1</smiles>

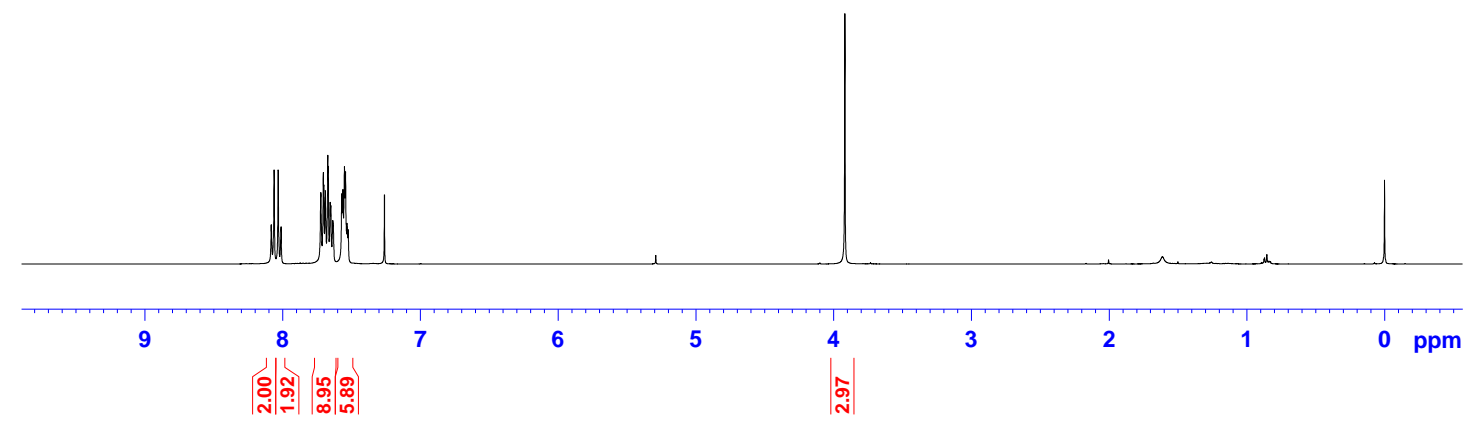


${ }^{13} \mathrm{C}$ NMR (100 MHz, $\left.\mathrm{CDCl}_{3}\right)$

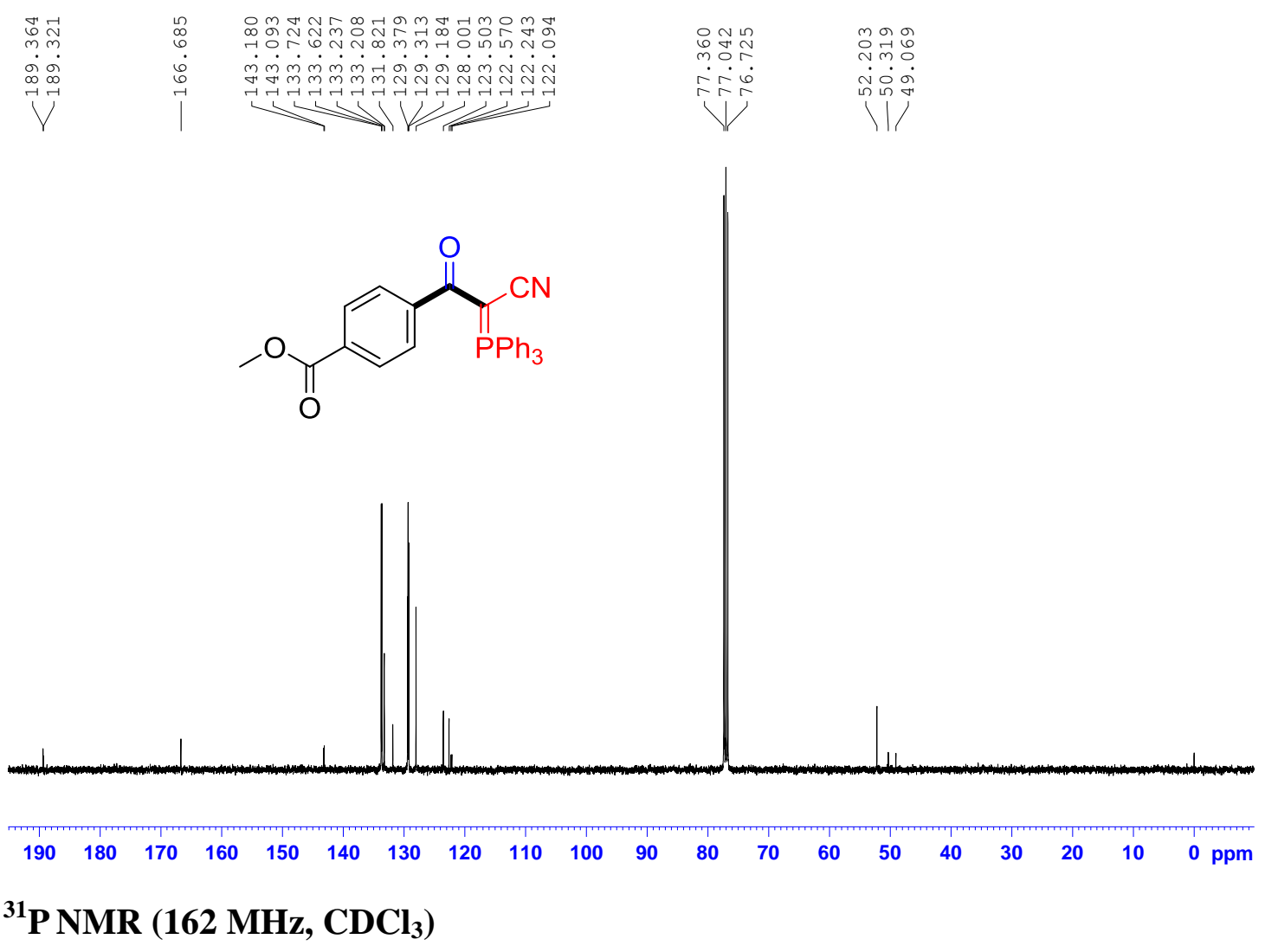

PNMR (162 MHz, $\left.\mathrm{CDCl}_{3}\right)$<smiles>COC(=O)c1ccc(C(=O)C(=P)C(C#N)Pc2ccccc2)cc1</smiles>

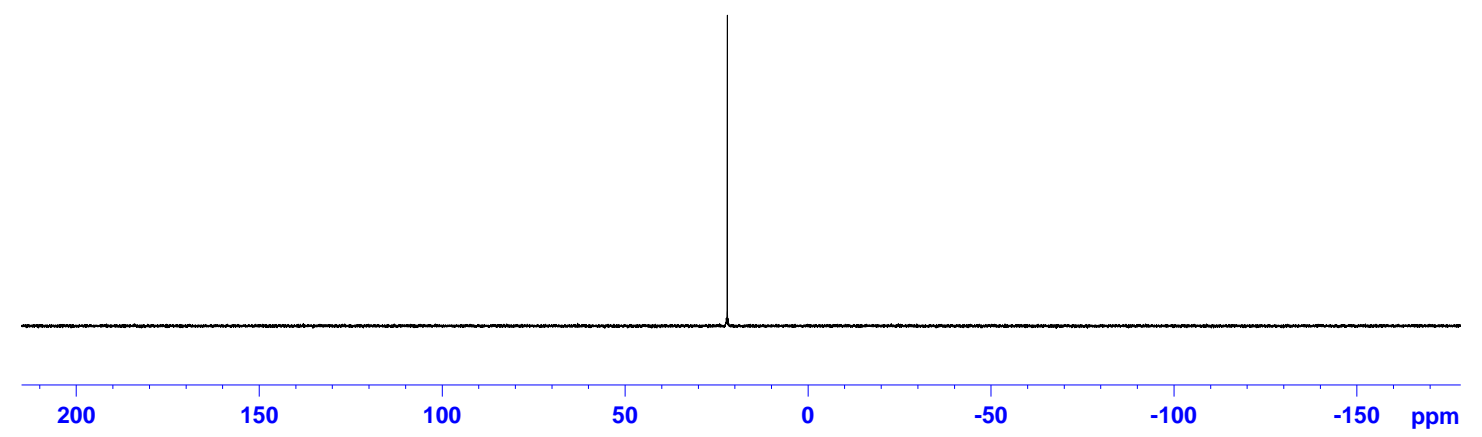


Methyl 4-(3-oxo-3-phenyl-2-(triphenylphosphoranylidene)propanoyl)benzoate 41:

${ }^{1} \mathrm{H}$ NMR (400 MHz, $\mathrm{CDCl}_{3}$ )
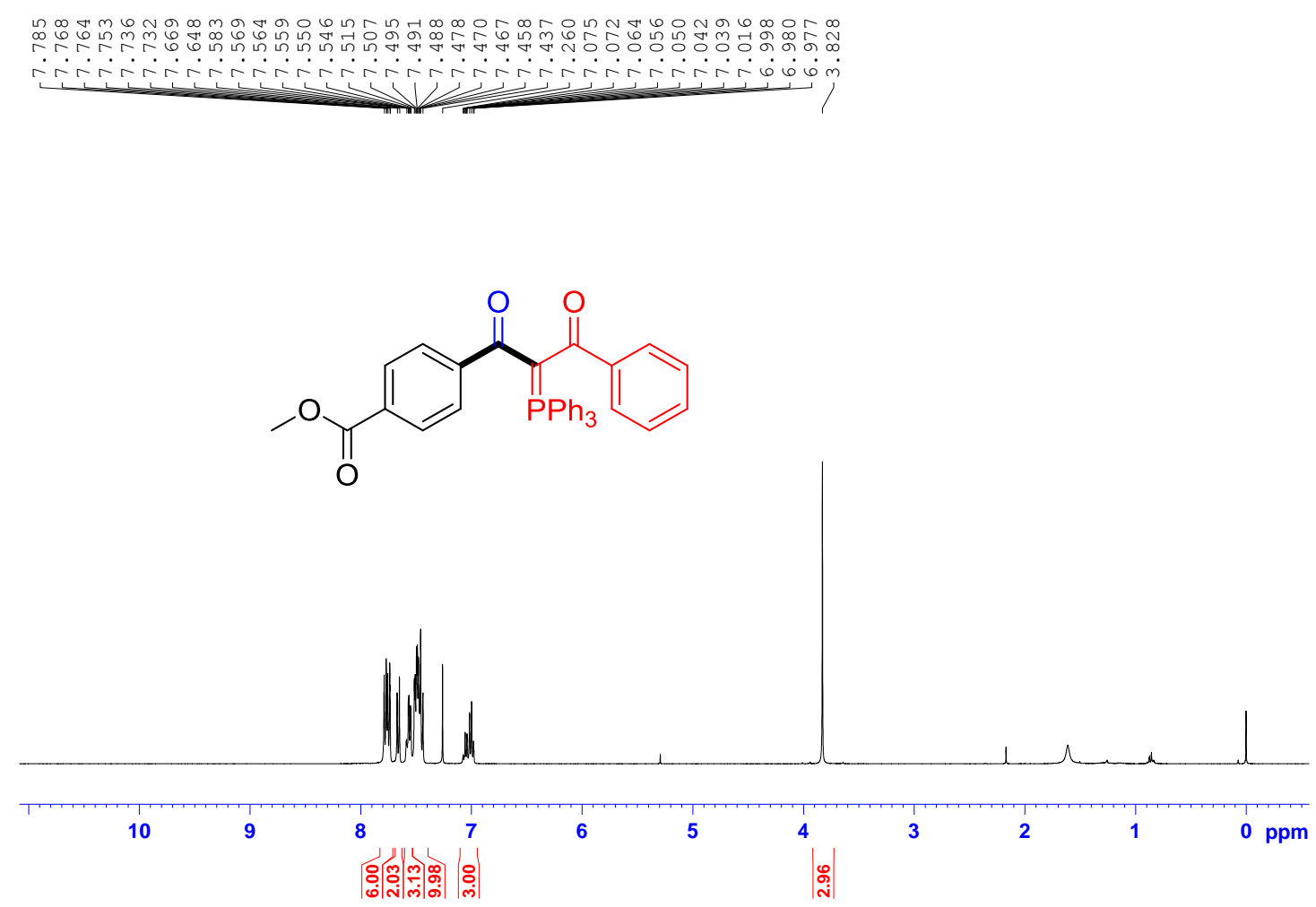

${ }^{13} \mathrm{C}$ NMR (100 MHz, $\mathrm{CDCl}_{3}$ )

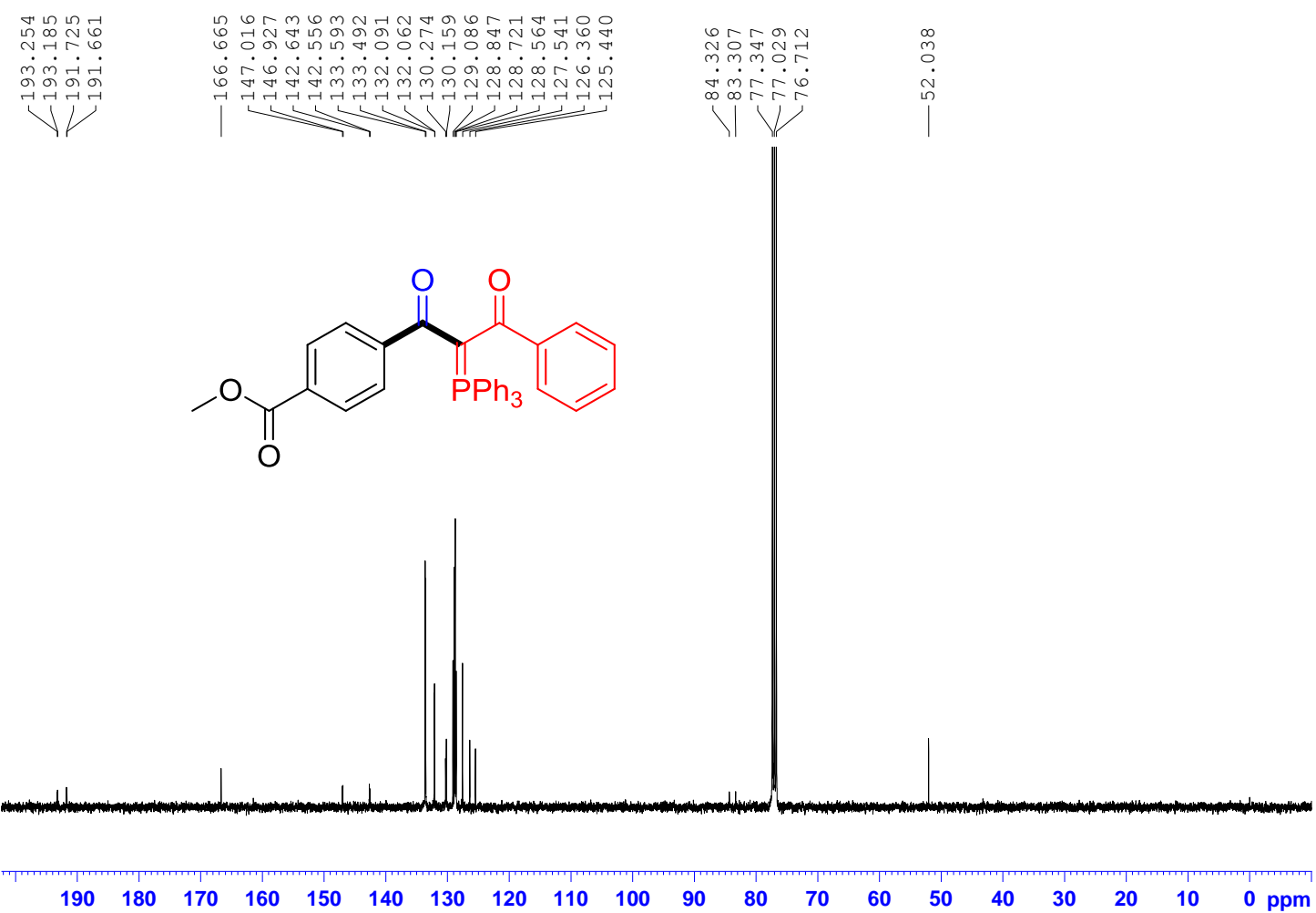


${ }^{31}$ P NMR (162 MHz, $\left.\mathrm{CDCl}_{3}\right)$<smiles>COC(=O)c1ccc(C(=O)C(=P)C(=O)c2ccccc2)cc1</smiles>

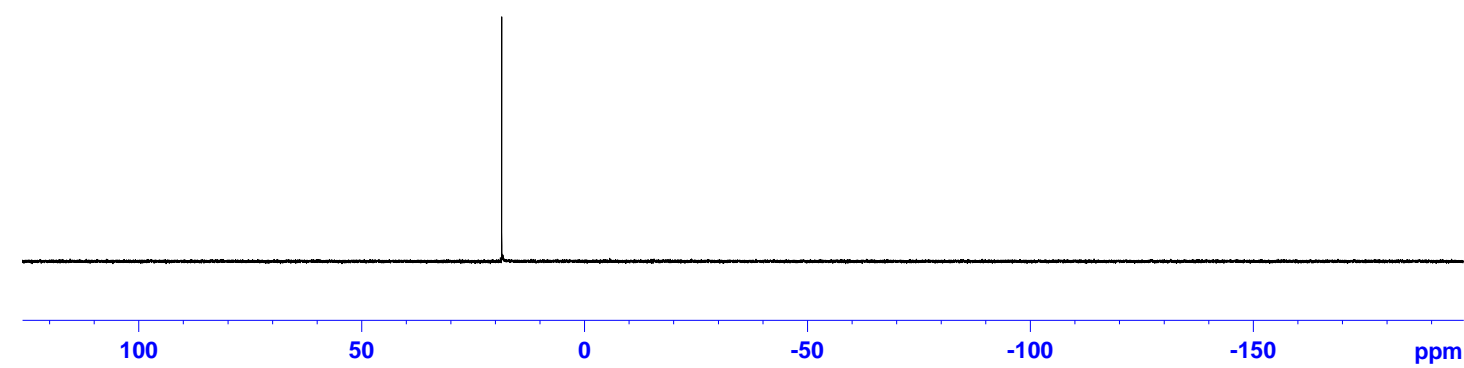

Methyl 4-(3-oxo-3-(p-tolyl)-2-(triphenylphosphoranylidene)propanoyl)benzoate 42: ${ }^{1} \mathrm{H}$ NMR (400 $\mathrm{MHz}, \mathrm{CDCl}_{3}$ )

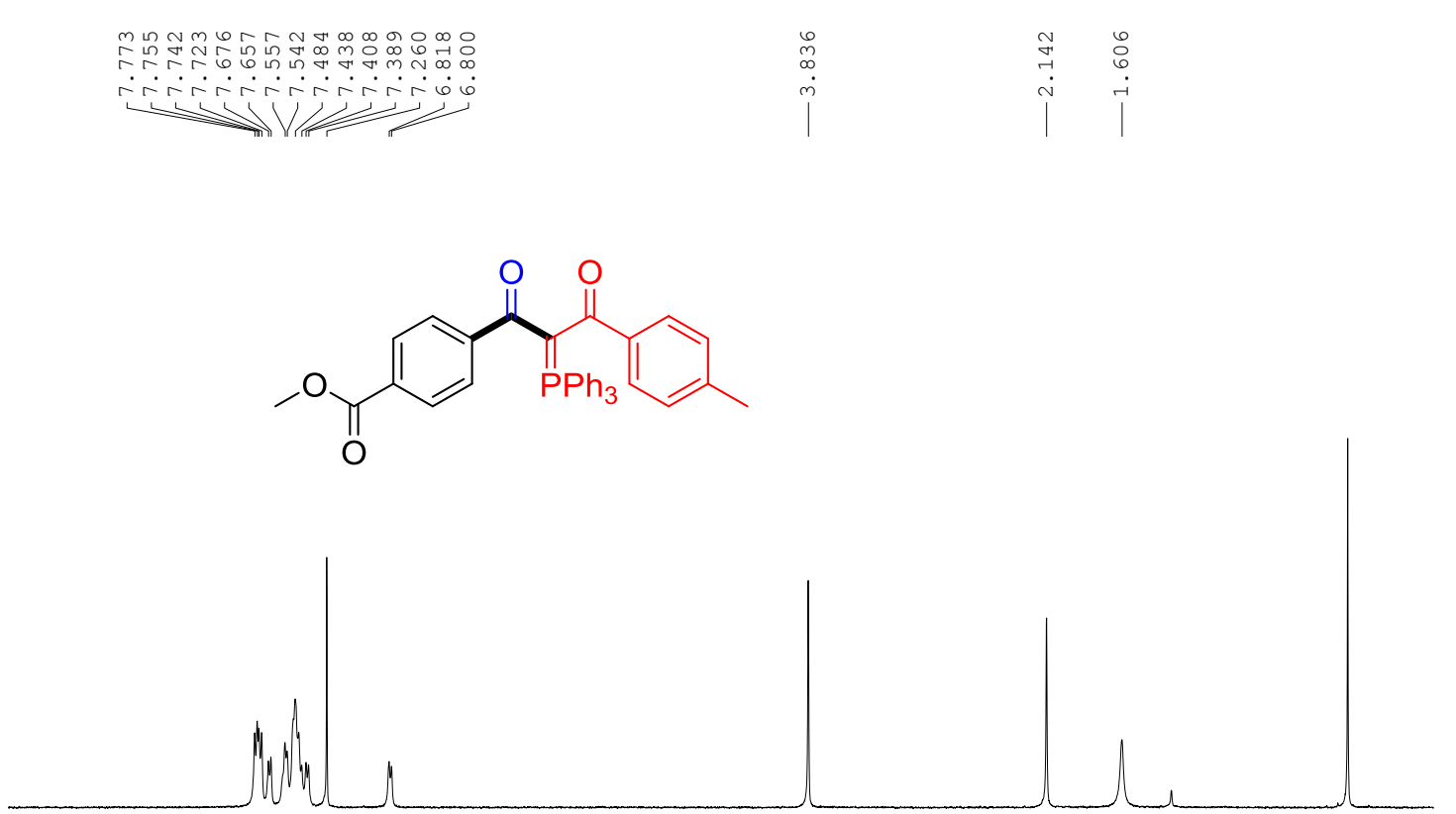

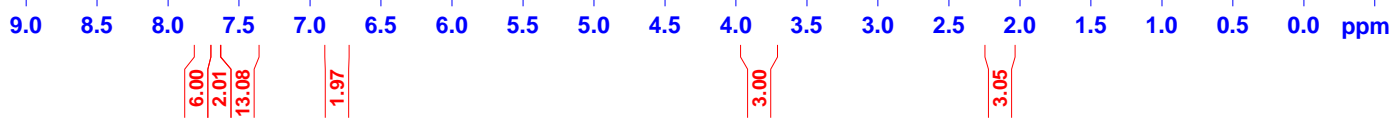




\section{${ }^{13} \mathrm{C}$ NMR (150 MHz, $\mathrm{CDCl}_{3}$ )}

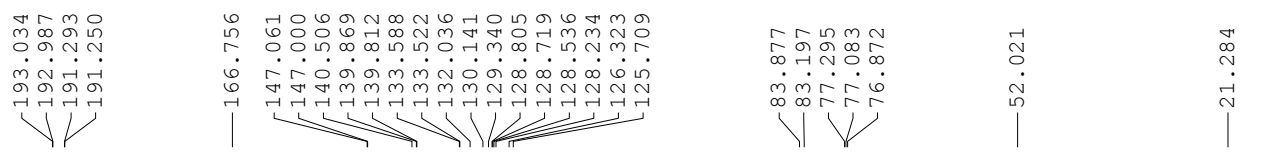<smiles>COC(=O)c1ccc(C(=O)C(=P)C(=O)c2ccc(C)cc2)cc1</smiles>

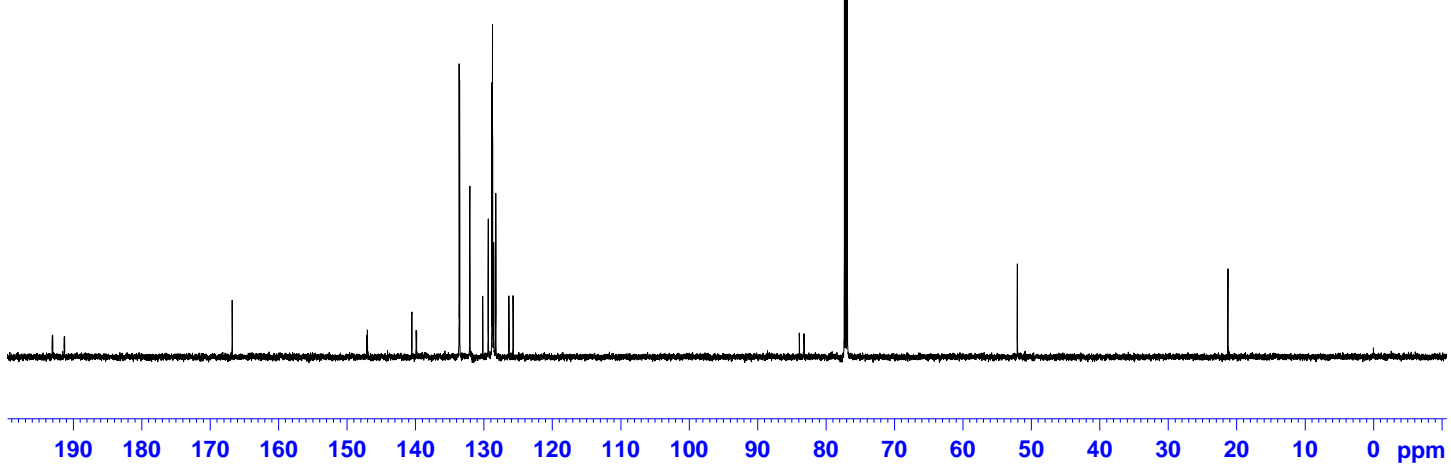

${ }^{31}$ P NMR (162 MHz, $\mathrm{CDCl}_{3}$ )<smiles>COC(=O)c1ccc(C(=O)C(=P)C(=O)c2ccc(C)cc2)cc1</smiles>

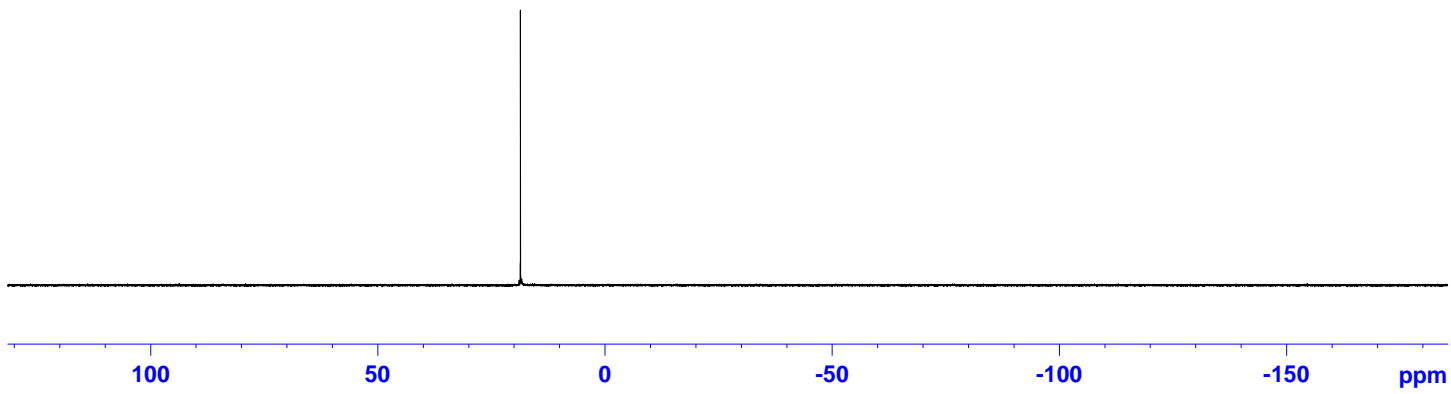


Methyl-4-(3-(4-methoxyphenyl)-3-oxo-2-(triphenylphosphoranylidene)propanoybenzoate 43: ${ }^{1} \mathrm{H}$ NMR (400 MHz, $\left.\mathrm{CDCl}_{3}\right)$
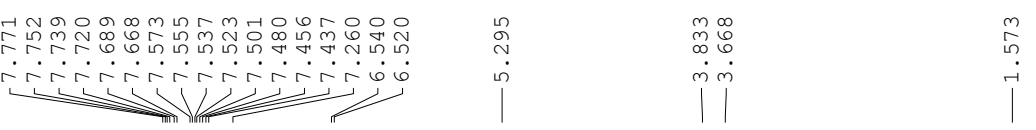<smiles>COC(=O)c1ccc(C(=O)C(=P)C(=O)c2ccc(OC)cc2)cc1</smiles>

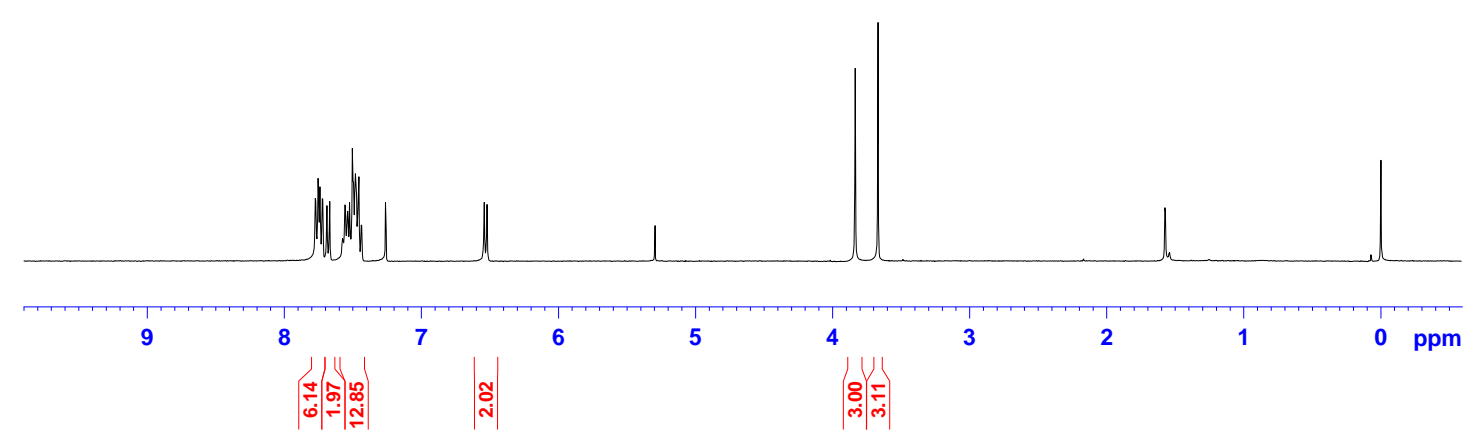

${ }^{13} \mathrm{C}$ NMR (100 MHz, $\left.\mathrm{CDCl}_{3}\right)$
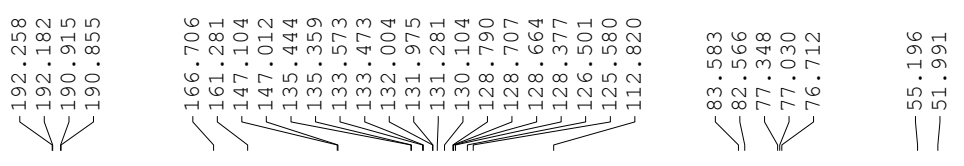<smiles>COC(=O)c1ccc(C(=O)C(=P)C(=O)c2ccc(OC)cc2)cc1</smiles>

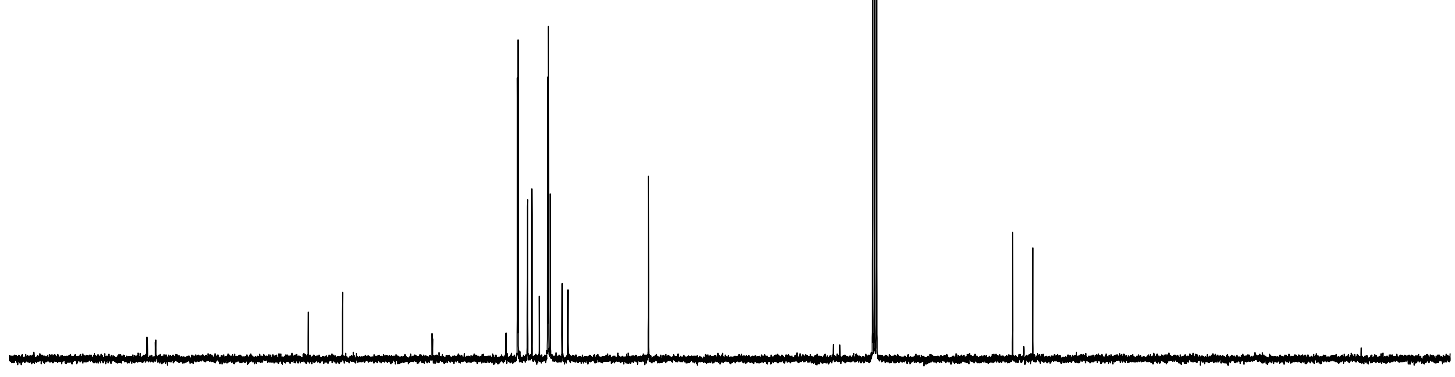

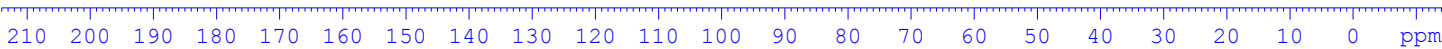


${ }^{31}$ P NMR (162 MHz, $\left.\mathrm{CDCl}_{3}\right)$<smiles>COC(=O)c1ccc(C(=O)C(=P)C(=O)c2ccc(OC)cc2)cc1</smiles>

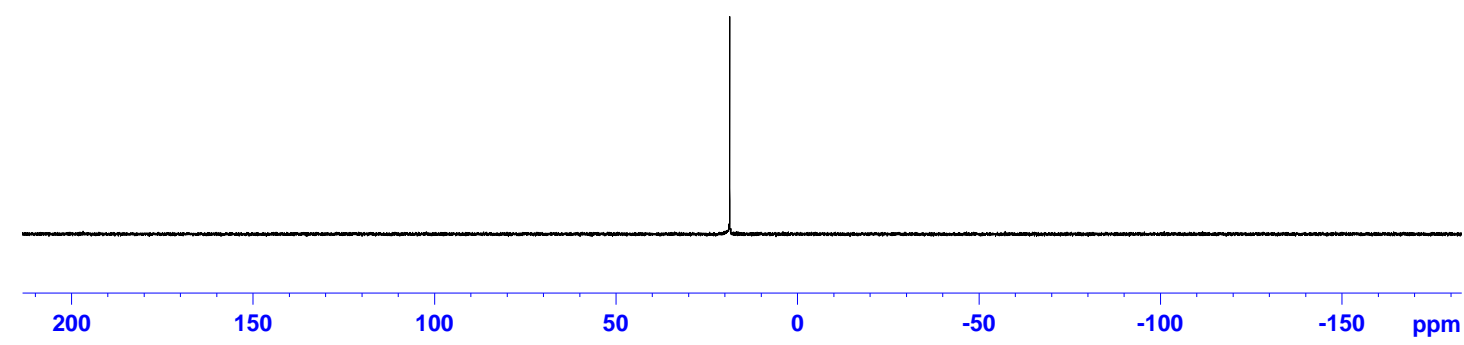

Methyl-4-(3-([1,1'-biphenyl]-4-yl)-3-oxo-2-(triphenylphosphoranylidene)propano -yl)benzoate 44: ${ }^{1} \mathrm{H}$ NMR (600 MHz, $\left.\mathrm{CDCl}_{3}\right)$

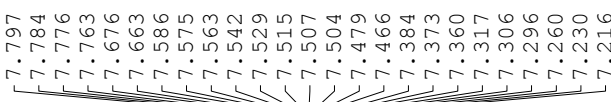<smiles>COC(=O)c1ccc(C(=O)C(=P)C(=O)c2ccc(-c3ccccc3)cc2)cc1</smiles>

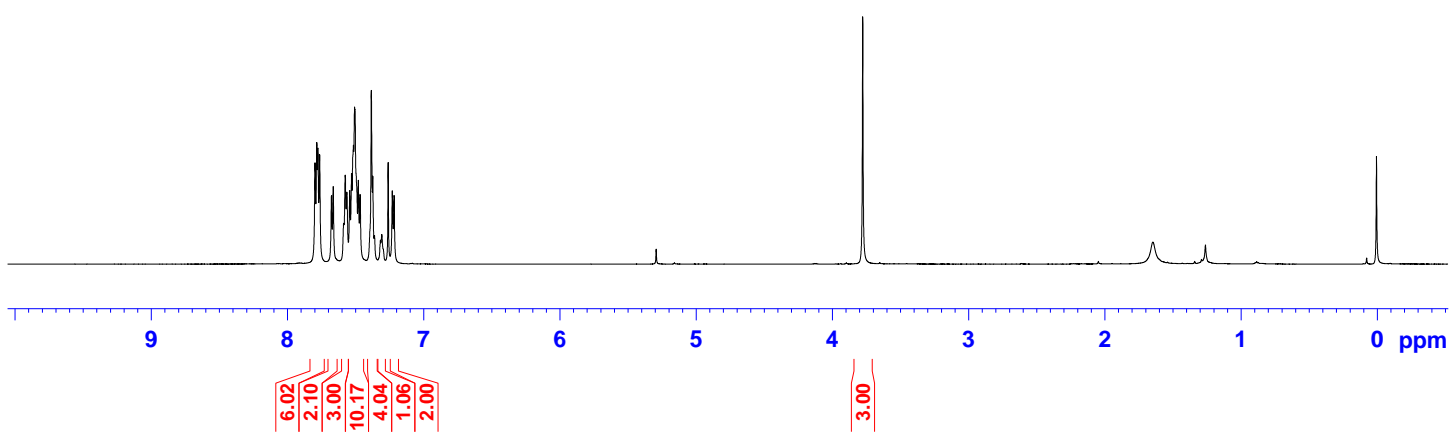




\section{${ }^{13} \mathrm{C}$ NMR (100 MHz, $\left.\mathrm{CDCl}_{3}\right)$}

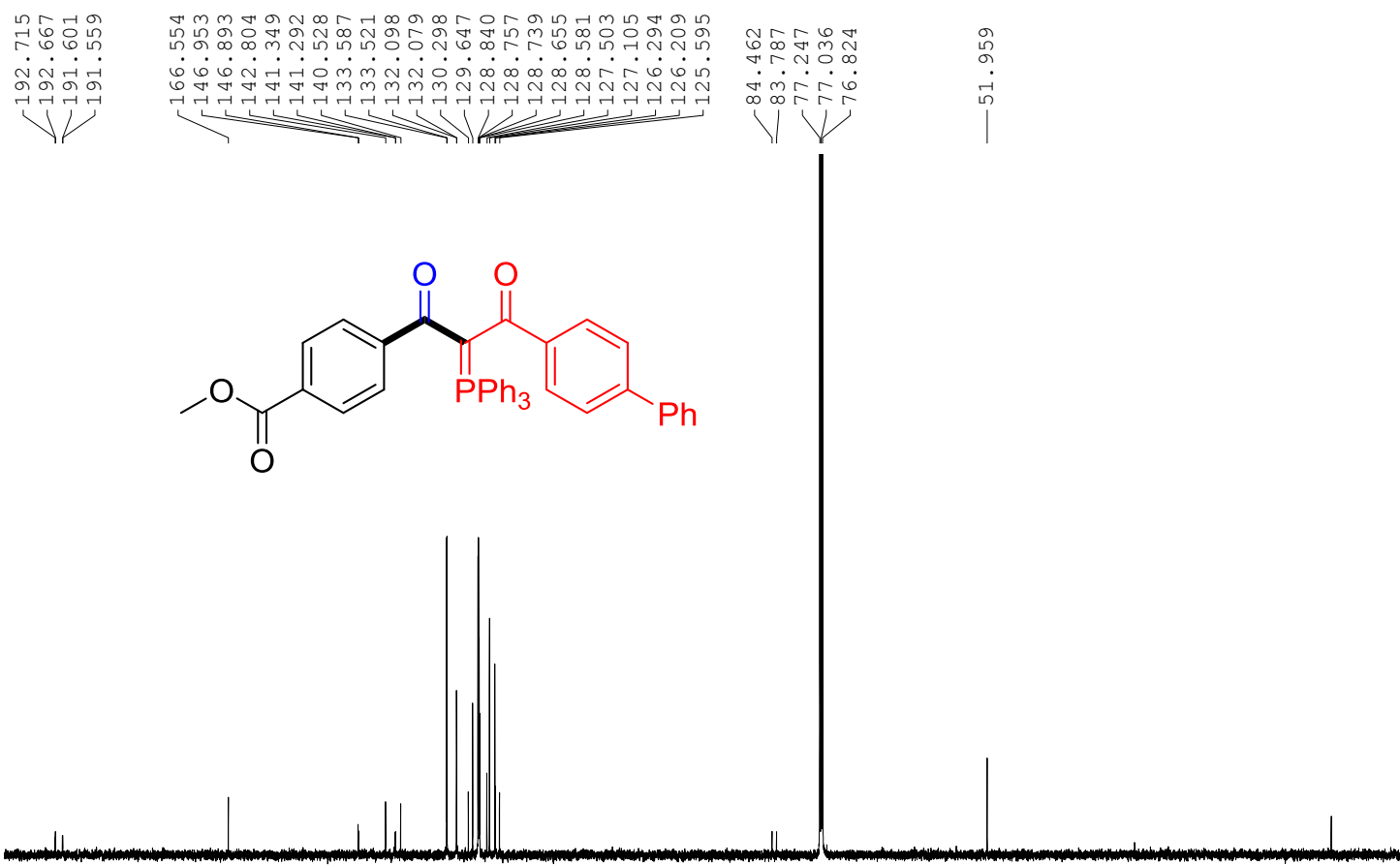

${ }^{31}$ P NMR (162 MHz, $\left.\mathrm{CDCl}_{3}\right)$<smiles>COC(=O)c1ccc(C(=O)C(=P)C(=O)c2ccc(-c3ccccc3)cc2)cc1</smiles>

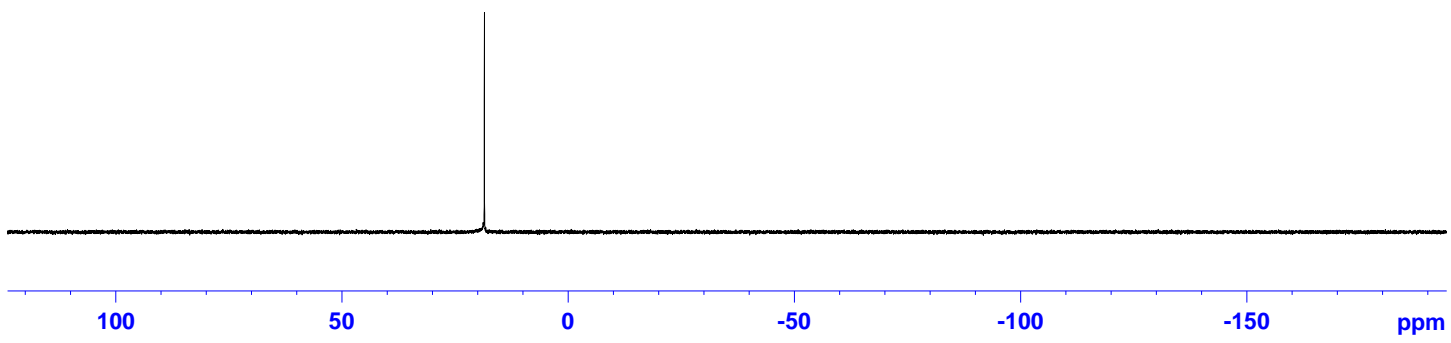


Methyl-4-(3-(4-cyanophenyl)-3-oxo-2-(triphenylphosphoranylidene)propanoyl)be -nzoate 45: ${ }^{1} \mathrm{H}$ NMR (400 $\left.\mathrm{MHz} \mathrm{CDCl}_{3}\right)$
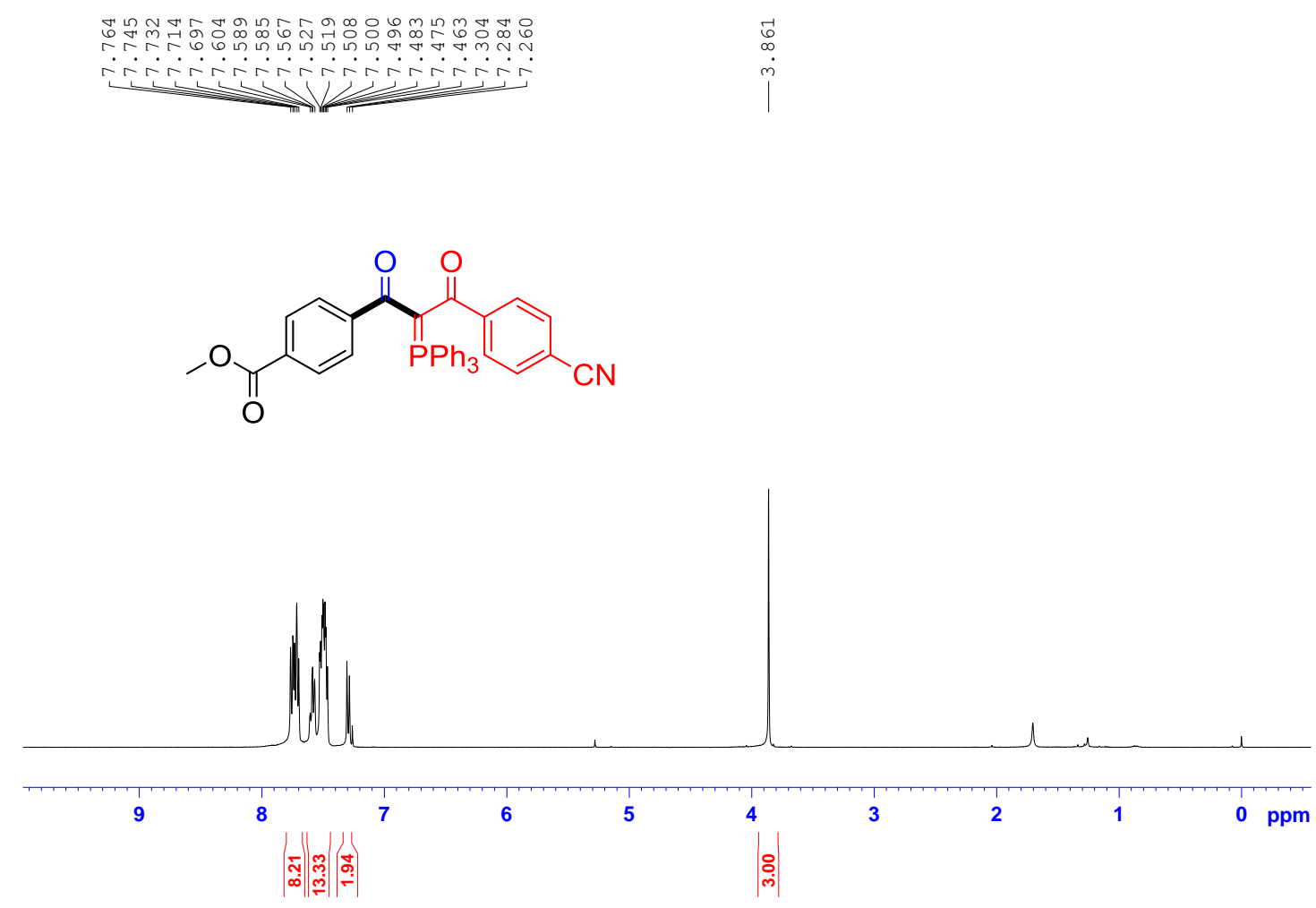

\section{${ }^{13} \mathrm{C}$ NMR (100 MHz, $\left.\mathrm{CDCl}_{3}\right)$}

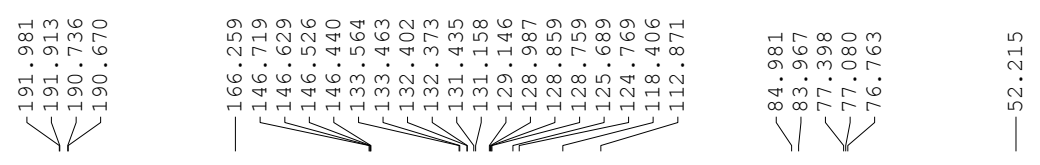

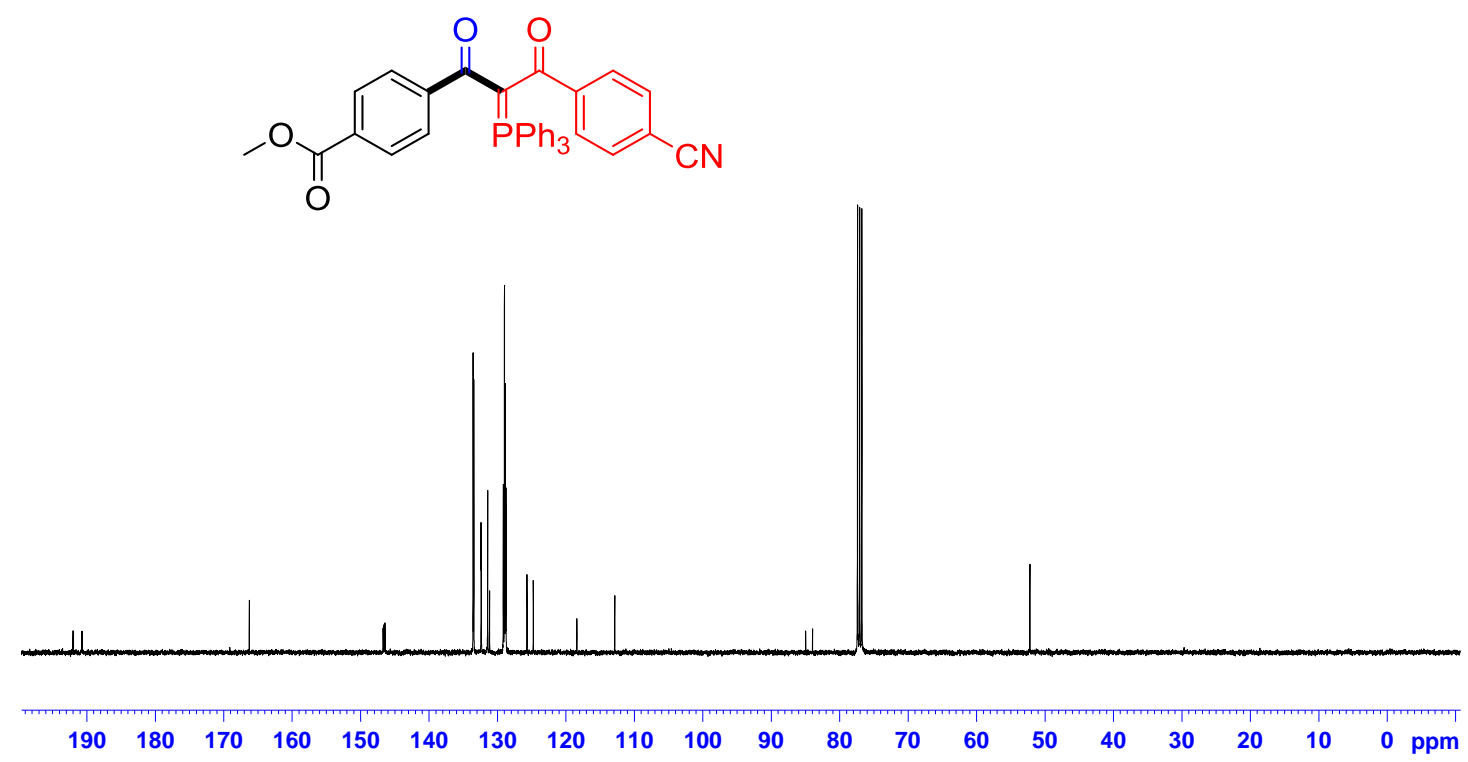


${ }^{31}$ P NMR (162 MHz, $\left.\mathrm{CDCl}_{3}\right)$
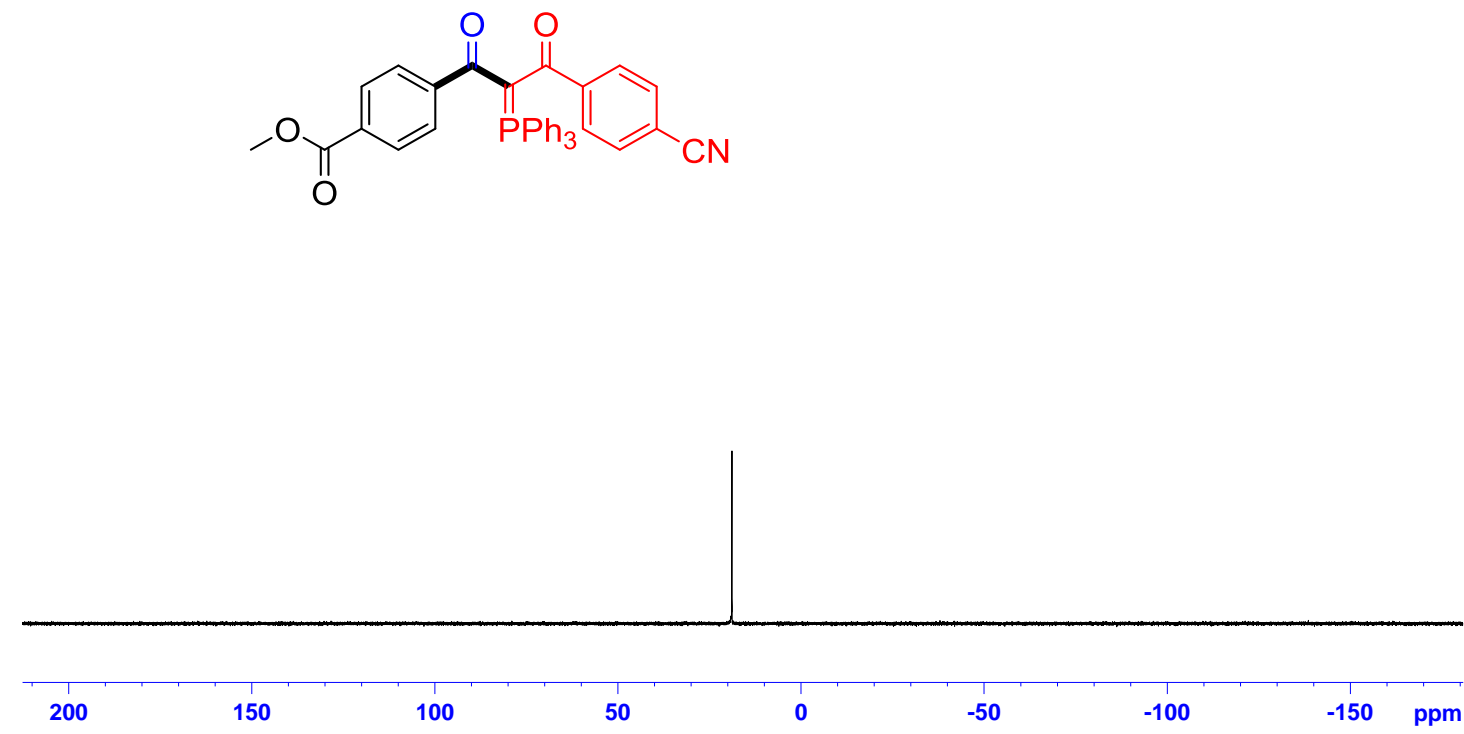

Methyl-4-(3-(naphthalen-2-yl)-3-oxo-2-(triphenylphosphoranylidene)propanoyl) benzoate 46: ${ }^{1} \mathrm{H}$ NMR (400 MHz, $\left.\mathrm{CDCl}_{3}\right)$

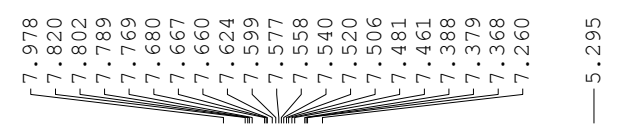<smiles>COC(=O)c1ccc(C(=O)C(=P)C(=O)c2ccc3ccccc3c2)cc1</smiles>

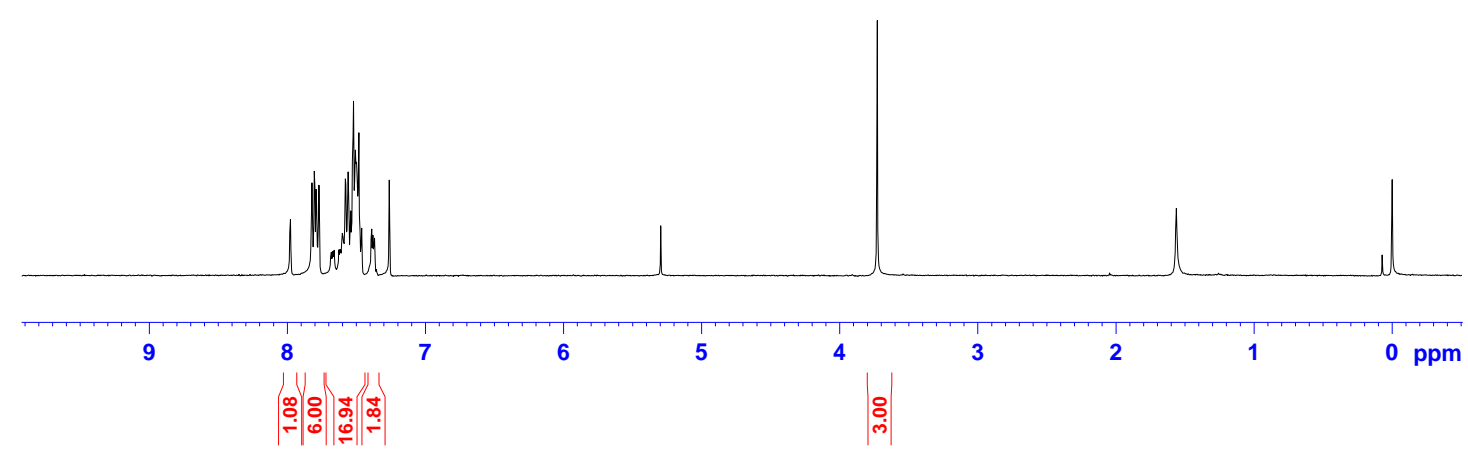




\section{${ }^{13} \mathrm{C}$ NMR (100 MHz, $\left.\mathrm{CDCl}_{3}\right)$}

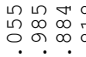 \\ अंगें}

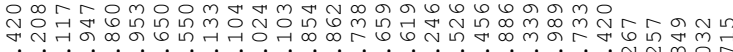

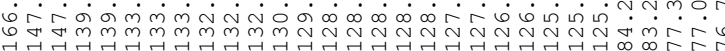
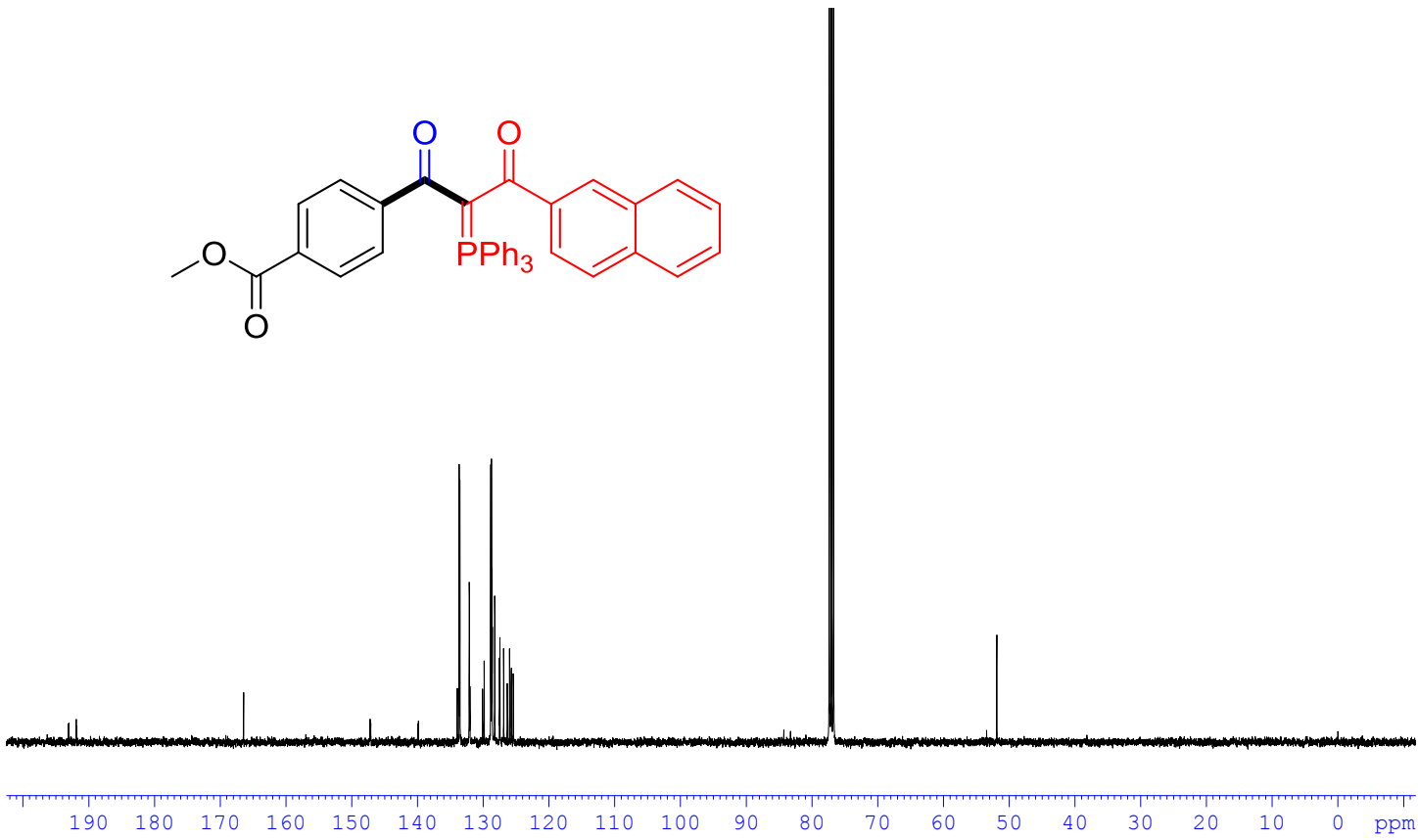

\section{${ }^{31}$ P NMR (162 MHz, $\left.\mathrm{CDCl}_{3}\right)$}<smiles>COC(=O)c1ccc(C(=O)C(=P)C(=O)c2ccc3ccccc3c2)cc1</smiles>

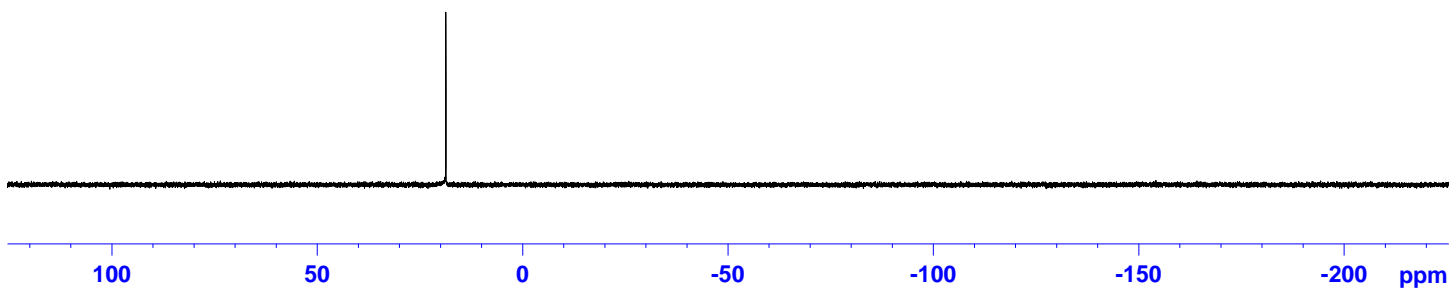


Methyl 3-phenylpropiolate 47:

${ }^{1}$ H NMR (400 MHz, CDCl $)$
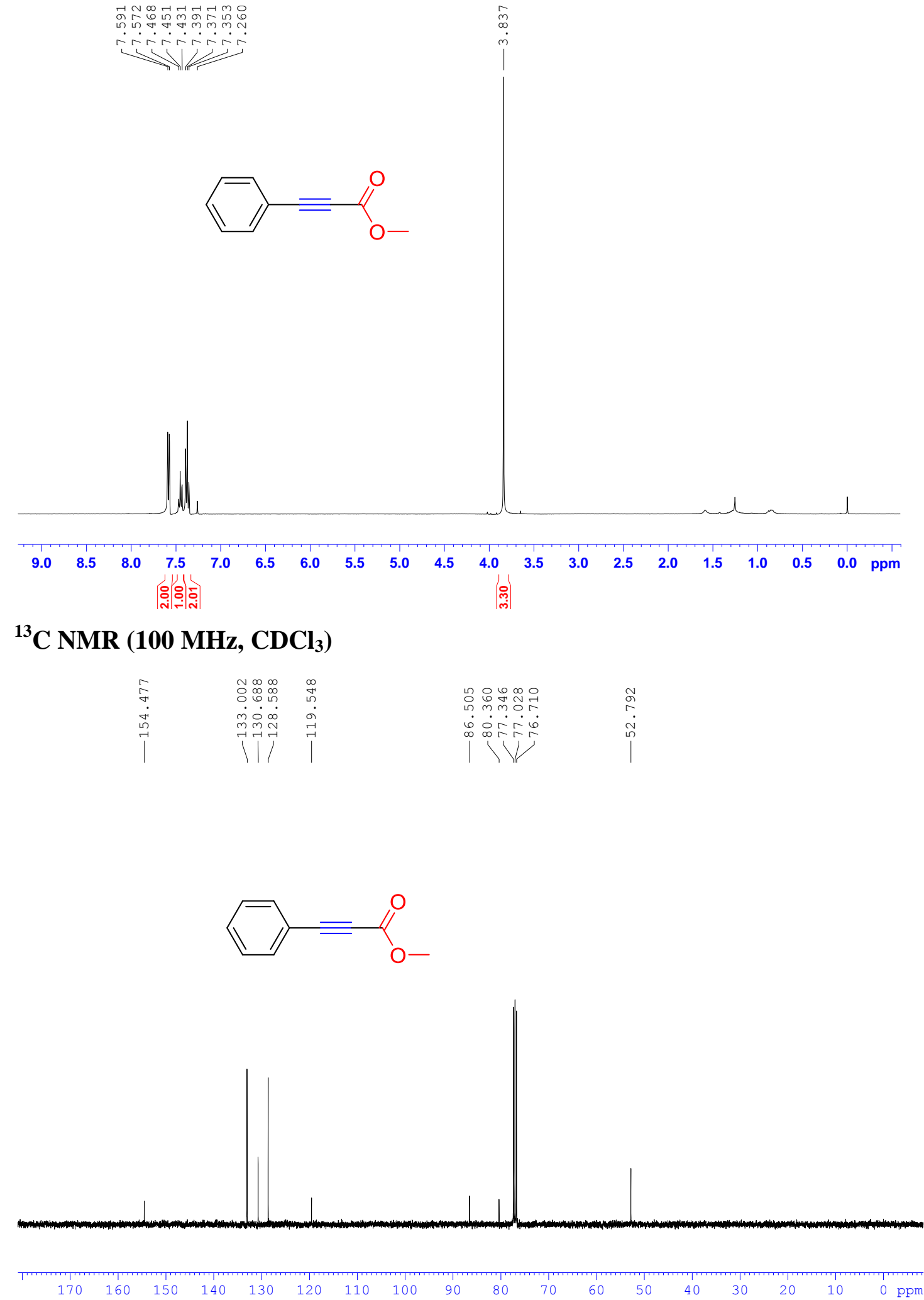
Ethyl 3-phenylpropiolate 48:

${ }^{1} \mathrm{H}$ NMR (400 MHz, $\left.\mathrm{CDCl}_{3}\right)$
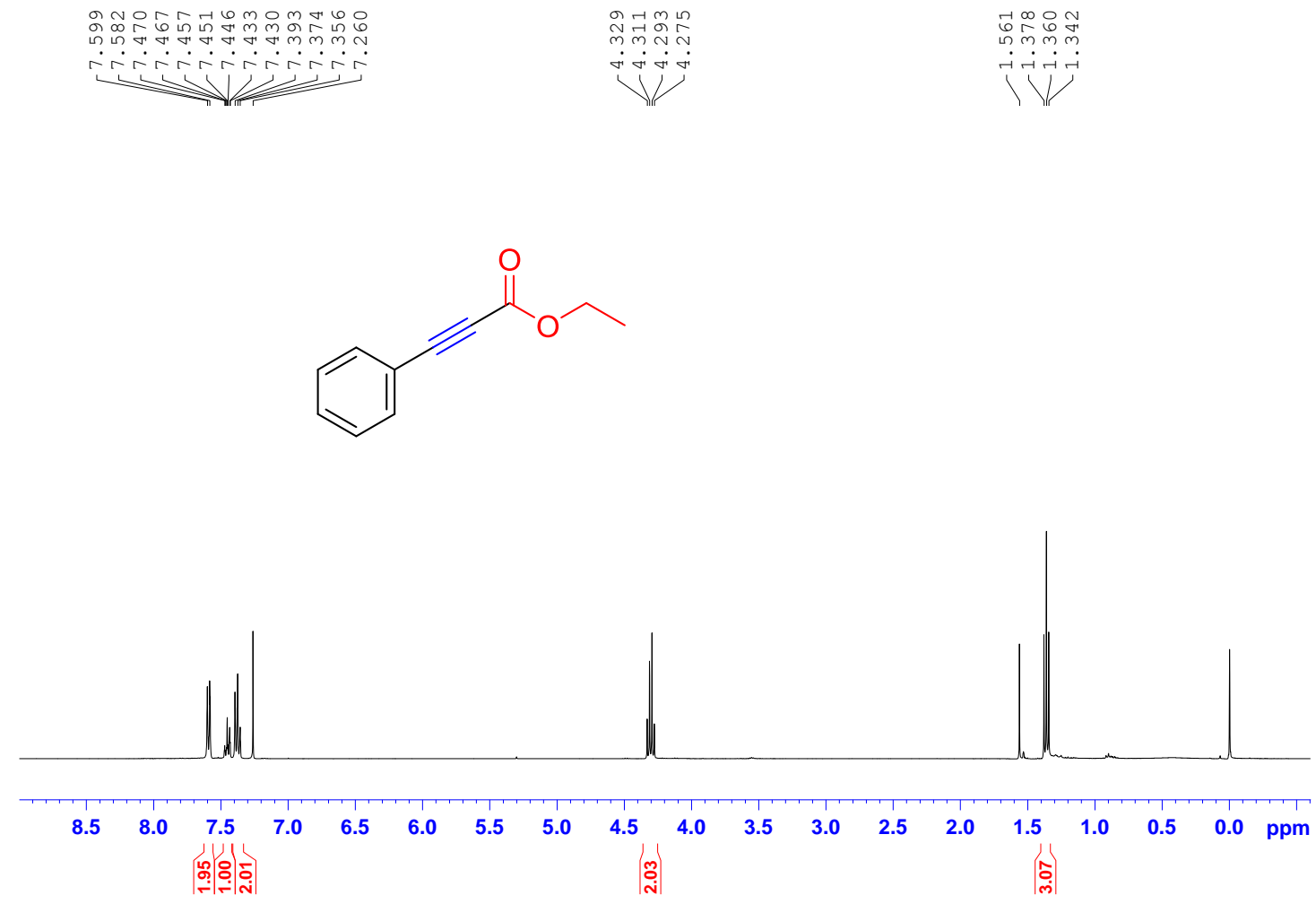

${ }^{13} \mathrm{C}$ NMR (100 MHz, $\left.\mathrm{CDCl}_{3}\right)$
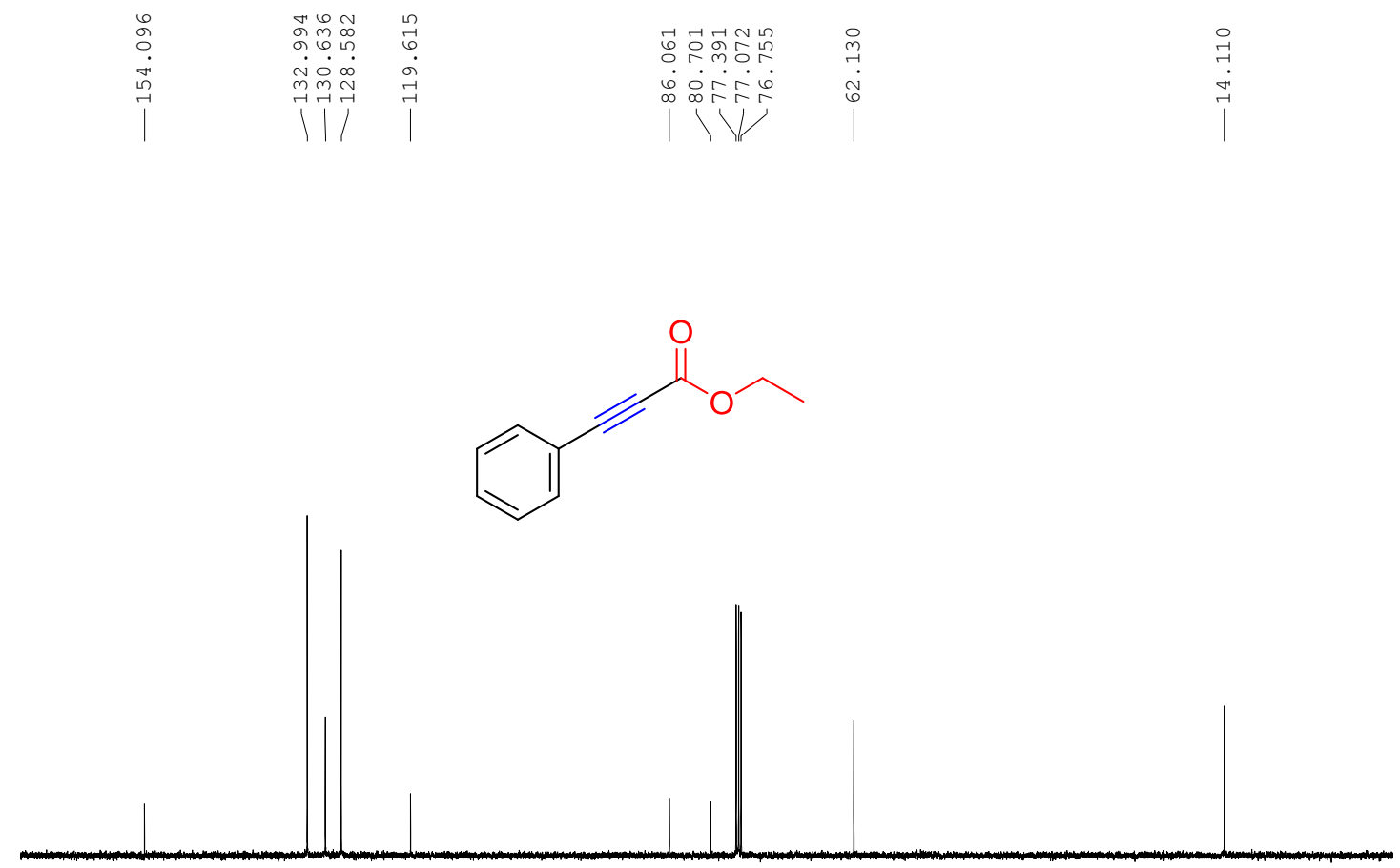

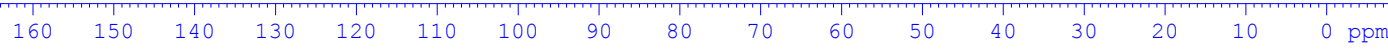


Methyl 3-(3-fluorophenyl)propiolate 49:

${ }^{1}$ H NMR (400 MHz, $\mathrm{CDCl}_{3}$ )
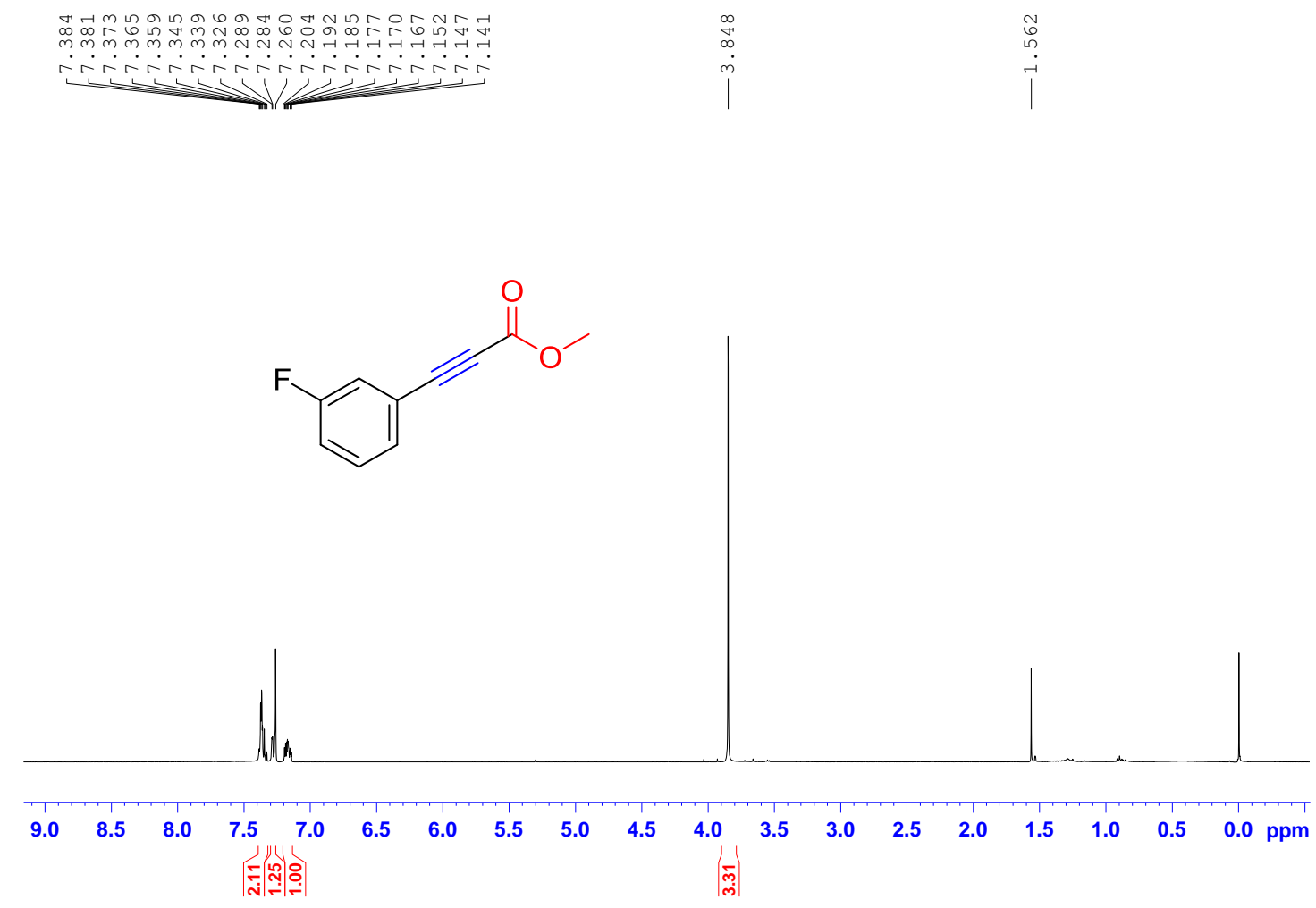

${ }^{13} \mathrm{C}$ NMR (100 MHz, $\left.\mathrm{CDCl}_{3}\right)$

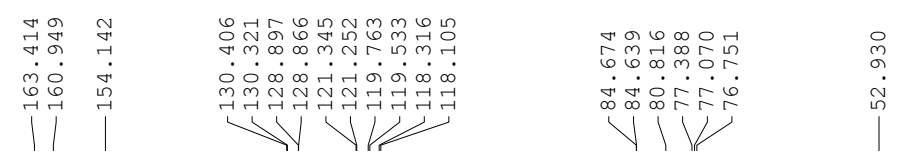

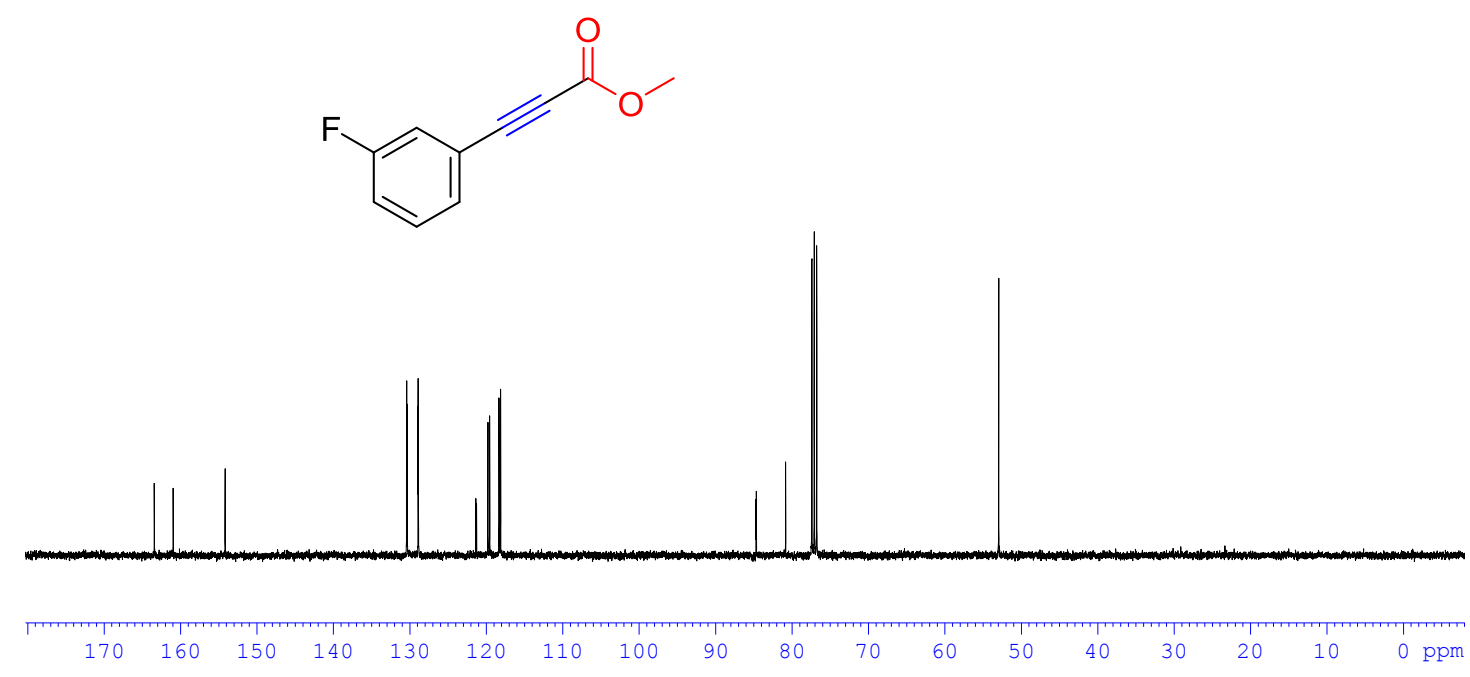


Methyl 3-(3-methoxyphenyl)propiolate 50:

${ }^{1} \mathrm{H}$ NMR (400 MHz, $\mathrm{CDCl}_{3}$ )

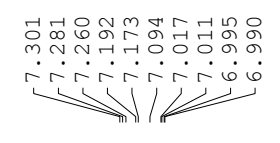<smiles>COC(=O)C#Cc1cccc(OC)c1</smiles>

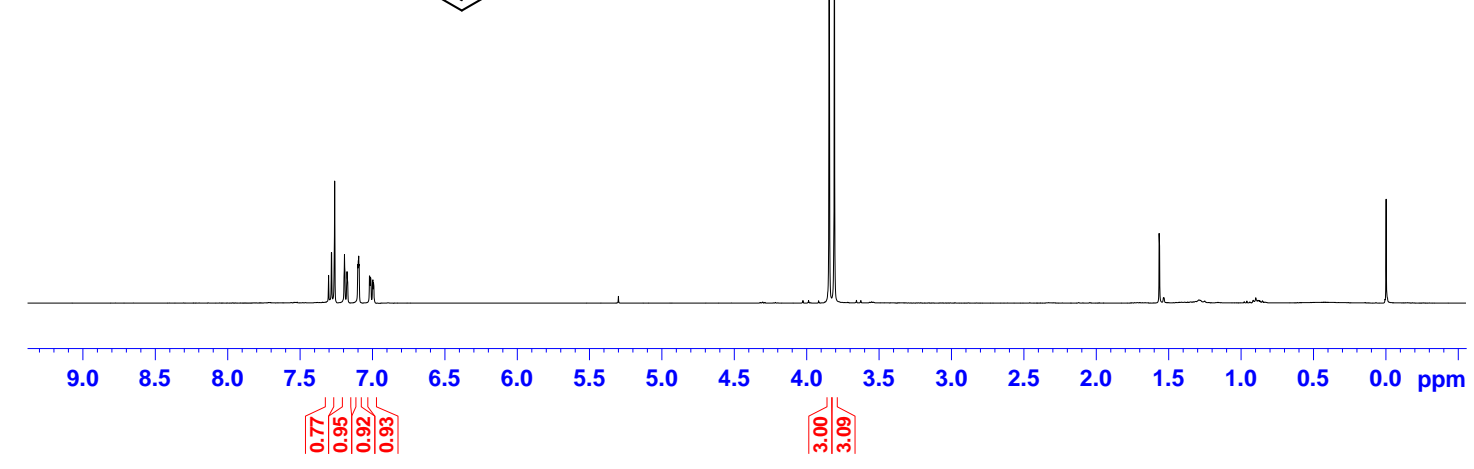

${ }^{13} \mathrm{C}$ NMR (100 MHz, $\left.\mathrm{CDCl}_{3}\right)$
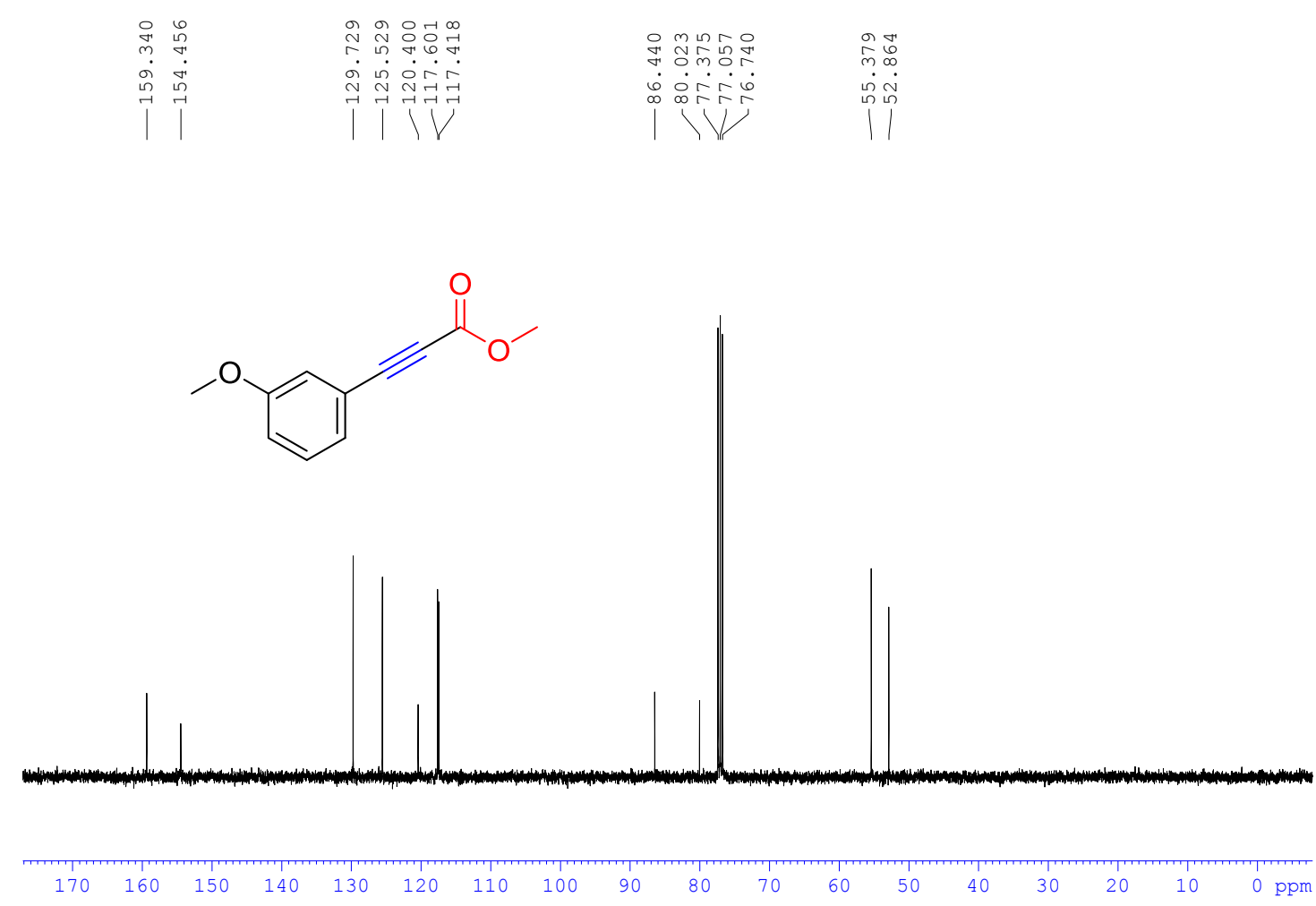
Methyl 3-(2-chloropyridin-4-yl)propiolate 51:

${ }^{1} \mathrm{H}$ NMR (400 MHz, $\mathrm{CDCl}_{3}$ )
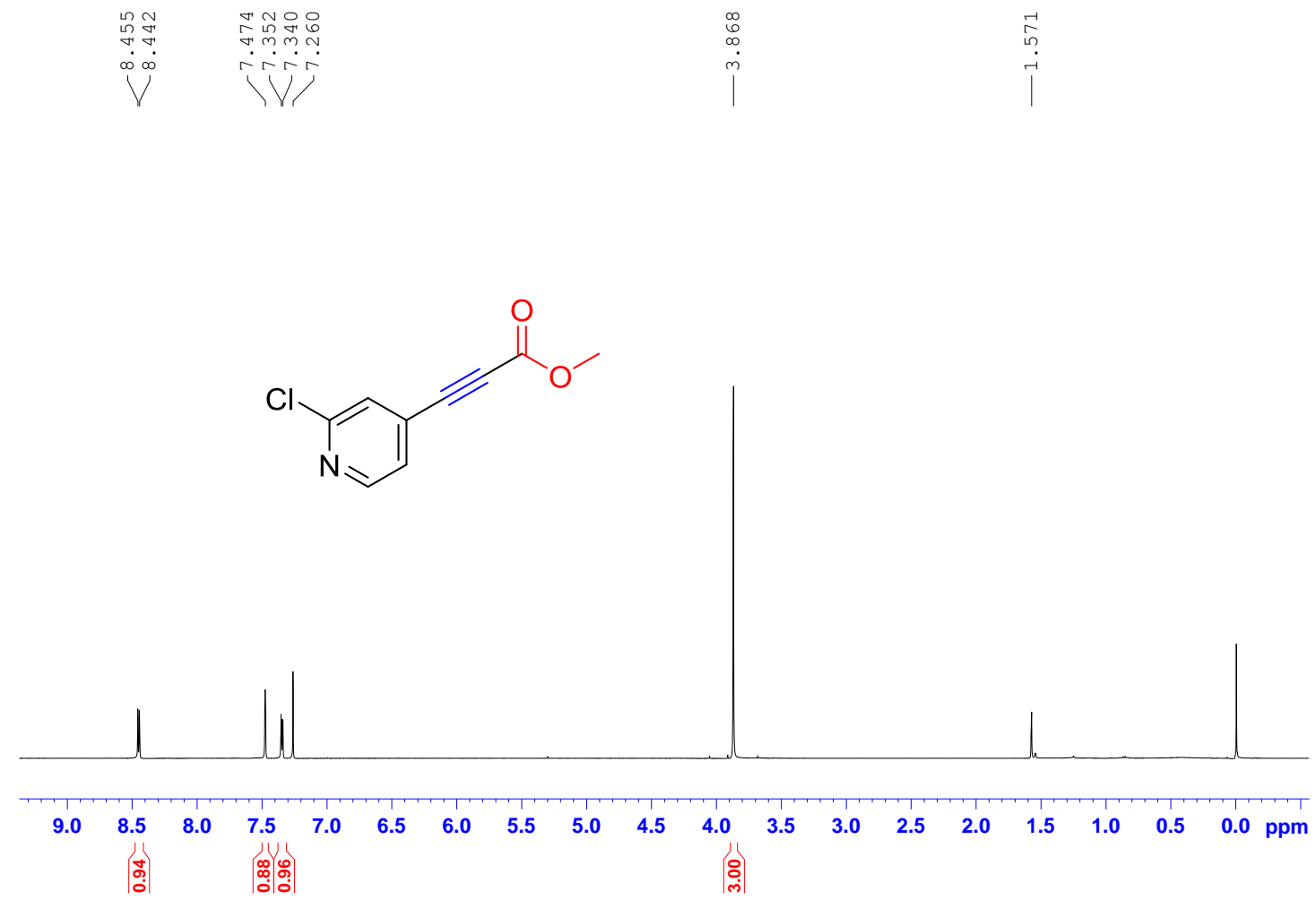

${ }^{13} \mathrm{C}$ NMR (100 MHz, $\left.\mathrm{CDCl}_{3}\right)$

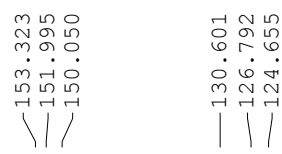

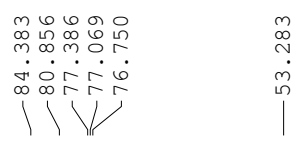<smiles>COC(=O)C#Cc1ccnc(Cl)c1</smiles>

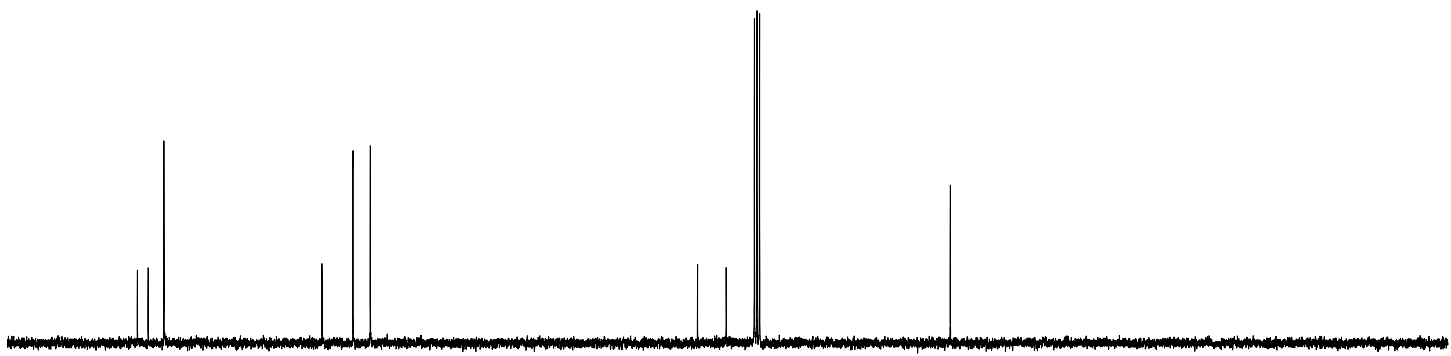

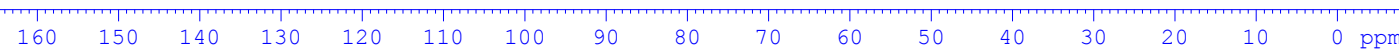

